Sistemas gradientes, decomposição de Morse e funções de Lyapunov sob perturbação

\author{
Éder Ritis Aragão Costa
}



SERVIÇO DE PÓS-GRADUAÇÃO DO ICMC-USP

Data de Depósito: 23 de Março de 2012

Assinatura:

\title{
Sistemas gradientes, decomposição de Morse e funções de Lyapunov sob perturbação
}

\author{
Éder Ritis Aragão Costa
}

Orientador: Prof. Dr. Alexandre Nolasco de Carvalho

Tese apresentada ao Instituto de Ciências Matemáticas e de Computação - ICMC-USP, como parte dos requisitos para obtenção do título de Doutor em Ciências - Matemática . VERSÃO REVISADA

USP - São Carlos

Março de 2012 
Ficha catalográfica elaborada pela Biblioteca Prof. Achille Bassi e Seção Técnica de Informática, ICMC/USP, com os dados fornecidos pelo(a) autor(a)

Aragão Costa, Éder Ritis

Sistemas gradientes, decomposição de Morse e funções de Lyapunov sob perturbação / Éder Ritis Aragã Costa; orientador Alexandre Nolasco de Carvalho. São Carlos, 2012.

$181 \mathrm{p}$.

Tese (Doutorado - Programa de Pós-Graduação em Matemática) -- Instituto de Ciências Matemáticas e de Computação, Universidade de São Paulo, 2012.

1. Sistemas gradientes. 2. Decomposição de Morse. 3. Funções de Lyapunov. 4. Estabilidade dos semigrupos gradientes sob perturbação. 5. Equações com acoplamento unilateral. I. Nolasco de Carvalho, Alexandre, orient. II. Título. 
Aos meus pais,

Vera e

Belmiro. 



\section{Agradecimentos}

Agradeço a todas as pessoas que, direta ou indiretamente, contribuíram para minha formação profissional e pessoal. Dentre elas, meus familiares e amigos que fiz durante toda minha vida. Principalmente, meus pais, Vera e Belmiro, e todos os meus professores, desde o da primeira série que pegou na minha mão e me ensinou a escrever as primeiras letras até meu orientador de doutorado, Alexandre Nolasco. Obrigado!

Finalmente, agradeço à FAPESP pelo apoio financeiro cedido e à CAPES pelo financiamento do estágio no exterior, sem os quais este trabalho não teria sido concluido. 

Neste trabalho investigamos a existência de uma função de Lyapunov associada a um sistema de tipo gradiente, semigrupos ou processos de evolução. Para isso, um estudo detalhado da teoria de Morse desempenha um papel decisivo. Como principal consequência deste estudo obtemos a estabilidade dos sistemas gradientes sob perturbação (autônoma ou não).

A aplicabilidade dos resultados abstratos que aqui discutimos é exemplificada estudando-se sistemas de equações diferenciais em espaços de Banach com acoplamento unilateral. 

In this work we investigated the existence of a Lyapunov function associated to a gradient-like system, semigroups or evolution processes. For that, a detailed study of Morse theory plays a central role. As the main consequence of this study we obtain the stability of gradient systems under perturbation (autonomous or not).

The applicability of the abstract results discussed here is exemplified by studying systems of differential equations in Banach spaces with unilateral coupling. 

Introdução $\quad$ xi

1 Atratores Globais 1

1.1 Primeiras definições . . . . . . . . . . . . . . . . . . 1

1.2 Conjuntos $\omega$-limites . . . . . . . . . . . . . . . . 8

1.3 Existência de atratores . . . . . . . . . . . . . . . . 15

2 Semigrupos Gradientes $\quad 19$

2.1 Função de Lyapunov . . . . . . . . . . . . . . . . . . . . . . . . 19

2.2 Estrutura dos semigrupos gradientes . . . . . . . . . . . . . . . . . 22

2.3 Existência de atratores para semigrupos gradientes . . . . . . . . 25

3 Semigrupos de tipo Gradiente $\quad 27$

3.1 Definição . . . . . . . . . . . . . . . . . . 27

3.2 Estabilidade por perturbação . . . . . . . . . . . . . . 28

4 Teoria de Morse-Conley 37

4.1 Pares atrator-repulsor . . . . . . . . . . . . . . . . 37

4.2 Função de Lyapunov para um par atrator-repulsor . . . . . . . . . . . . 45

4.3 Decomposição de Morse . . . . . . . . . . . . . . . . . 50

4.4 Equivalência entre as noções de semigrupos gradiente e de tipo gradiente 59

5 Estabilidade da função de Lyapunov $\quad \mathbf{6 5}$

5.1 Continuidade da família de atratores . . . . . . . . . . . . . . . 65

5.2 Convergência das funções de Lyapunov . . . . . . . . . . . . . . . . 68

6 Níveis de energia de um semigrupo gradiente $\quad 77$

6.1 Estrutura dos níveis . . . . . . . . . . . . . . . . . 77 
6.2 Perturbação de níveis . . . . . . . . . . . . . . . . . . . . . . . . . 84

7 Decomposição de Morse não autônoma $\quad 91$

7.1 Processos de evolução . . . . . . . . . . . . . . . . . . . . . . . 92

7.2 Processos de evolução de tipo gradiente . . . . . . . . . . . . . . . 96

7.3 Decomposição de Morse não autônoma . . . . . . . . . . . . . . . . . . 98

7.4 Função de Lyapunov para um processo de evolução de tipo gradiente 108

7.5 Perturbação não autônoma de um semigrupo gradiente . . . . . . . . . 119

7.5.1 Convergência das funções de Lyapunov . . . . . . . . . . . . . . 132

8 Aplicação a problemas com acoplamento unilateral 141

8.1 Teoria abstrata . . . . . . . . . . . . . . . . . 142

8.2 A desigualdade de Lojasiewicz-Simon . . . . . . . . . . . . . . . . 150

8.3 Aplicação da desigualdade de Lojasiewicz-Simon a problemas abstratos 158

8.3.1 Equações de primeira ordem . . . . . . . . . . . . . . . . 158

8.3 .2 Equações de segunda ordem . . . . . . . . . . . . . . 166

9 Conclusões e problemas em aberto $\quad 175$

$\begin{array}{ll}\text { Referências Bibliográficas } & 179\end{array}$ 


\section{Introdução}

A análise das propriedades qualitativas dos semigrupos em espaços de fases gerais (espaços de Banach infinito dimensionais ou espaços métricos arbitrários), recebeu muita atenção durante as últimas quatro decádas. Em particular, o estudo dos conjuntos compactos invariantes e atratores desenvolveu uma ampla e profunda linha de pesquisa, dando informações cruciais sobre um número crescente de fenômenos de distintos ramos da Ciência, como Física, Biologia, Economia, Engenharia e outros.

Quando se mostra que um dado sistema dinâmico possui atrator global, todo seu comportamento assintótico pode ser descrito, fazendo-se uma análise detalhada da dinâmica interna deste conjunto compacto invariante. Para se entender a dinâmica interna do atrator global, um estudo cuidadoso da estrutura geométrica (e sua estabilidade por perturbação) aparece como um fator fundamental. Provavelmente, o resultado mais geral nesta linha é o que se conhece como o "Teorema Fundamental dos Sistemas Dinâmicos", sugerido em [17] a partir dos resultados de [12], o qual estabelece que qualquer grupo (ou "fluxo") em um espaço métrico compacto, decompõe o espaço em partes invariantes isoladas recorrentes por cadeia e as conexões entre elas. Na terminologia de [12], isto é o que se chama uma decomposição de Morse de um conjunto compacto invariante (confira a Definição 4.3.1), e foi considerado em diferentes marcos, como no caso dos grupos em [12], e também dos semigrupos em espaços compactos como em [20], ou ainda, em espaços topológicos não compactos como em [15], [18], [19].

Por outro lado, muito recentemente, foi introduzido em [8] os denominados semigrupos de tipo gradiente (veja a Definição 3.1.1), como um conceito intermediário entre os semigrupos gradientes (aqueles que possuem uma função de Lyapunov) e os semigrupos que possuem atrator de tipo gradiente (aqueles possuindo atrator escrito como a reunião dos conjuntos instáveis de seus conjuntos invariantes isolados).

Neste texto, em um primeiro momento, trabalharemos com um semigrupo de tipo gradiente em um espaço métrico arbitrário e construiremos uma função de Lyapunov 
para ele, mostrando que todo sistema de tipo gradiente é efetivamente um sistema gradiente. Nossos resultados evitam qualquer hipótese de compacidade associada ao espaço de fase sobre o qual atuam os semigrupos que consideramos. Por outro lado, nossas provas, ainda que inspiradas em trabalhos clássicos, como [12],[20], são diferentes das aí incluídas e portanto são novas.

Para a construção da função de Lyapunov, primeiramente provaremos que a família disjunta dos conjuntos invariantes isolados de um semigrupo de tipo gradiente em um espaço métrico geral, pode ser reordenada de tal maneira a dar lugar a uma decomposição de Morse do atrator. Com provas bastante intuitivas, centradas na dinâmica do semigrupo e, por exemplo, não fazendo uso das noções de recorrência por cadeia, que é um conceito bastante tradicional nesta teoria.

Um refinamento dos resultados de [12] nos levará a uma definição de função de Lyapunov generalizada, não simplesmente definida no atrator, mas sim, em todo o espaço de fase e também associada a uma família de conjuntos invariantes isolados arbitrária, não necessariamente um conjunto de equilíbrios.

Por outro lado, em um segundo momento, trabalharemos no contexto não autônomo da teoria dos sistemas dinâmicos, onde estudaremos a mesma natureza de problemas estudados no caso autônomo. Trabalharemos, portanto, com processos de evolução de tipo gradiente (veja a Definição 7.2.4) buscando introduzir, para estes, as noções de decomposição de Morse e de função de Lyapunov e então, motivados pela teoria autônoma, provar, baixo certas condições, a existência de uma função de Lyapunov para tais processos.

Cabe dizer que, não encontramos, na literatura atual, nenhum resultado sobre a caracterização da existência de uma função de Lyapunov baseada na dinâmica de um semigrupo ou de um processo não autônomo, como os que provamos aqui.

Com o objetivo de apresentar, passo a passo, as técnicas que costumam ser usadas no estudo dos semigrupos não lineares e também introduzir as ferramentas necessárias para o entendimento do resultado principal de equivalência entre os conceitos de semigrupos gradiente e tipo gradiente e sobre o tratamento do mesmo problema no marco não autônomo, ordenamos o texto da seguinte forma:

No primeiro capítulo, apresentamos definições dos conceitos básicos nesta teoria e estabelecemos as primeiras propriedades dos sistemas dinâmicos autônomos, como as importantes propriedades dos conjuntos $\omega$-limites de um sistema assintoticamente compacto, o que será crucial para o primeiro resultado sobre existência de atrator global que apresentamos.

Os semigrupos gradientes serão definidos no capítulo dois, mas definidos de maneira mais geral do que a que se encontra, por exemplo, em [13]. Em outras palavras, aqui os 
consideramos associados a uma família finita disjunta de conjuntos invariantes isolados qualquer, não necessariamente um conjunto finito de pontos de equilíbrio. Para estes semigrupos estudaremos, em detalhes, a estrutura de seu atrator, provando que este se escreve como reunião dos conjuntos instáveis dos invariantes isolados, isto é, que seu atrator é de tipo gradiente. Para finalizar o capítulo, estabelecemos um critério de existência de atrator para semigrupos gradientes, baseado no resultado mais geral contido no primeiro capítulo.

Uma vez que se conhecem os principais resultados dos primeiros capítulos, estamos em condições de analizar o conteúdo verdadeiramente novo deste trabalho, e mais precisamente, quando se define uma nova classe de sistemas dinâmicos (os de tipo gradiente, segundo [8]), que são sistemas dinâmicos que possuem a dinâmica de um sistema gradiente. Nestas condições, o objetivo do capítulo três é proporcionar condições suficientes que garantam a estabilidade por perturbação deste novo conceito.

O capítulo quatro contém as ferramentas que serão decisivas para estabelecer nosso resultado de equivalência. Nele definiremos a noção de decomposição de Morse de um atrator em termos dos pares atrator-repulsor. A importância destes pares é que se podem construir, para eles, uma espécie de "função de Lyapunov" com propriedades muito especiais, o que será crucial para obter a função de Lyapunov clássica para os semigrupos de tipo gradiente, provando assim o principal resultado deste trabalho, no marco autônomo. Além disso, forneceremos condições suficientes para que, ao perturbar um dado semigrupo gradiente, estas funções convirjam uniformemente sobre compactos, o que é o objetivo do capítulo cinco, e também que tais funções sejam diferenciáveis ao longo de soluções.

No capítulo seis, apresentamos outra forma de obter uma decomposição de Morse para o atrator de um semigrupo de tipo gradiente, em termos de um conceito novo que chamamos nível de energia, e que nos dá informação adicional sobre o comportamento assintótico das soluções do sistema, "empacotando" aqueles conjuntos invariantes isolados que possuem características dinâmicas semelhantes. Além disso, fornecemos condições suficientes para a estabilidade por perturbação destes níveis, o que ocorre, por exemplo, quando as conexões entre os invariantes isolados se mantêm.

O tratamento dos problemas não autônomos começa no capítulo sete, onde apresentamos as definições de processo de evolução, famílias invariantes isoladas, o conceito de atrator pullback e processo de evolução de tipo gradiente, o qual é nosso principal objeto de interesse. Aqui, também buscaremos dar sentido ao conceito não autônomo de uma decomposição de Morse, o que será, assim como aconteceu no caso dos semigrupos, de fundamental importância para a construção das funções de Lyapunov para os processos de tipo gradiente. Procuraremos reproduzir no contexto dos processos, de 
forma consistente, todas as ferramentas que foram cruciais para a solução autônoma do problema, bem como, por exemplo, a idéia de pares atrator-repulsor.

Devemos adiantar que, no estudo não autônomo dos problemas relacionados à decomposição de Morse e as funções de Lyapunov, algumas restrições deverão ser feitas, o que nos obrigará a buscar exemplos onde verificam-se tais exigências. Mais precisamente, mostraremos que perturbações não autônomas de semigrupos gradientes, verificam as condições exigidas, evidenciando, assim, que as imposições feitas são bastante razoáveis.

Um exemplo mais concreto, por assim dizer, de aplicação de nossos resultados abstratos, está contido no capítulo oito. Lá estudamos um problema com acoplamento unilateral, onde, usando nossa teoria abstrata, forneceremos condições para que o mesmo "gere" um semigrupo de tipo gradiente e, portanto, gradiente. Além disso, no marco dos espaços de Hilbert, consideraremos a possibilidade de haver uma quantidade infinita de pontos estacionários, quando estudamos a Desigualdade de Lojasiewicz-Simon e estendemos alguns dos resultados contidos em [24].

Finalizamos o texto destacando as conclusões que obtivemos neste trabalho e os artigos que a partir dele foram escritos, além de apontar certos problemas que ficaram em aberto durante o desenvolvimento da tese, abrindo a possibilidade para possíveis trabalhos futuros. 


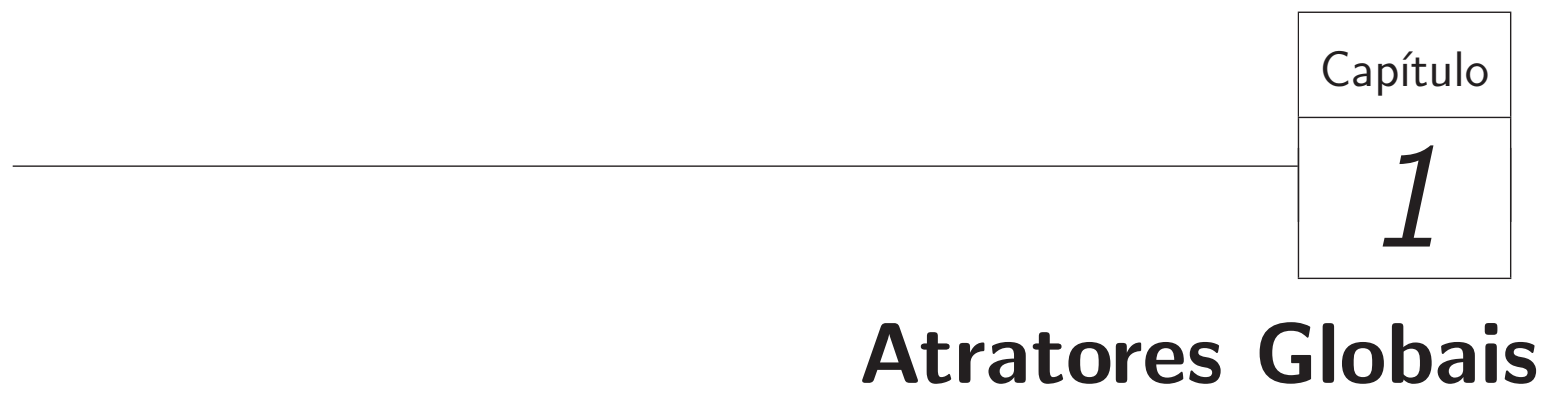

Neste capítulo apresentaremos os conceitos básicos e desenvolvemos os primeiros resultados da teoria dos sistemas dinâmicos autônomos, a fim de que possamos tratar, posteriormente, o problema de perturbação de sistemas gradientes.

Deixemos aqui registrado, antes de começarmos a discussão, que para nós, sempre e em todos os casos, o símbolo $\mathbb{N}$ representará o conjunto dos número naturais, que tem como seu primeiro elemento o número um, ou seja, $\mathbb{N}=\{1,2,3, \cdots\}$.

\subsection{Primeiras definições}

Seja $X$ um espaço métrico com distância $d: X \times X \rightarrow \mathbb{R}$.

Definição 1.1.1. Uma família $\{T(t): t \geq 0\}$ de aplicações contínuas do espaço métrico $X$ em si mesmo chama-se um semigrupo em $X$, quando verificam-se as seguintes três propriedades:

(i) $T(0)=I$, a aplicação identidade do espaço $X$.

(ii) $T(t+s)=T(t) T(s)$ qualquer que seja o par de números reais não negativos $t$ e $s$ (propriedade de semigrupo).

(iii) A aplicação $[0, \infty) \times X \ni(t, x) \longmapsto T(t) x \in X$ é uma aplicação contínua.

Na definição anterior, estamos considerando o conjunto $[0, \infty) \times X$ dotado da topologia produto.

Semigrupos são também chamados sistemas dinâmicos autônomos e, neste texto, utilizaremos indistintamente ambas nomenclaturas. 
Observemos que, pela propriedade de semigrupo, a família de aplicações $\{T(t): t \geq$ $0\}$ é comutativa com respeito à composição, visto que quaisquer que sejam $t, s \geq 0$, temos $T(s) T(t)=T(s+t)=T(t+s)=T(t) T(s)$.

Observemos ainda que, se $\{T(t): t \geq 0\}$ é um semigrupo com a propriedade de que a aplicação $T(t): X \rightarrow X$ é um homeomorfismo para cada $t>0$, então definindo, para $t<0, T(t):=T(-t)^{-1}: X \rightarrow X$, a família de aplicações contínuas $\{T(t): t \in \mathbb{R}\}$ costuma ser chamada um grupo em $X$. Aqui, contudo, trabalharemos apenas com semigrupos, ou seja, com o caso onde não se tem, necessariamente, $T(t): X \rightarrow X$ invertível para cada $t>0$.

O conceito de espaço métrico, em conjunto com sua estrutura topológica, e a noção de semigrupo, como definimos acima, são os únicos ingredientes teóricos que utilizaremos para desenvolver toda a teoria que compõe a parte autônoma inicial deste trabalho.

Fixemos, de uma vez por todas, um semigrupo $\{T(t): t \geq 0\}$, que também indicamos por $T(\cdot)$, em um espaço métrico $X:=(X, d)$, que as vezes chamamos o espaço de fase do semigrupo.

Neste contexto, podemos dar as seguintes definições necessárias para que possamos definir o que se entende por atrator global para um semigrupo:

Diz-se que um subconjunto $A \subset X$ é invariante pelo semigrupo $\{T(t): t \geq 0\}$, ou simplesmente invariante, quando para todo $t \geq 0$ tem-se $T(t) A=A$.

Seja $\left(A_{\lambda}\right)_{\lambda \in L}$ uma família de subconjuntos de $X$ invariantes pelo semigrupo $T(\cdot)$, então sua reunião, $A:=\bigcup_{\lambda \in L} A_{\lambda}$, é também invariante.

Com efeito, basta observar que para todo $t \geq 0, T(t)\left(\bigcup_{\lambda \in L} A_{\lambda}\right)=\bigcup_{\lambda \in L} T(t) A_{\lambda}$, e que para todo $\lambda \in L, T(t) A_{\lambda}=A_{\lambda}$.

Por outro lado, a interseção de uma família de conjuntos invariantes não é, necessariamente, um conjunto invariante. Entretanto, se para cada $t>0$ a aplicação $T(t): X \rightarrow X$ é injetiva então, é fácil ver, do fato de que $T(t)\left(\bigcap_{\lambda \in L} A_{\lambda}\right)=\bigcap_{\lambda \in L} T(t) A_{\lambda}$, qualquer que seja a família $\left(A_{\lambda}\right)_{\lambda \in L}$ de subconjuntos de $X$, que a interseção de invariantes é invariante.

A noção de conjunto invariante está intimamente ligada a de solução global, cuja definição é a seguinte:

Diz-se que uma aplicacão $\xi: \mathbb{R} \rightarrow X$ é uma solução global para o semigrupo $\{T(t): t \geq 0\}$, quando para todo $t \geq 0$ e todo $\tau \in \mathbb{R}$,

$$
T(t) \xi(\tau)=\xi(t+\tau)
$$


Se $\xi: \mathbb{R} \rightarrow X$ é uma solução global, sua imagem indica-se pelo símbolo $\gamma(\xi)$, ou seja, $\gamma(\xi):=\{\xi(\tau): \tau \in \mathbb{R}\}$, e chama-se a órbita de $\xi$ ou, as vezes, para evitar confusões, diz-se ainda órbita global da solução $\xi$. Quando $\xi(0)=x \in X$, costuma-se dizer que $\xi$ é uma solução global que passa pelo ponto $x$ ou, simplesmente, uma solução global por $x$.

Verifica-se que toda órbita global de um semigrupo é invariante. Com efeito, dado $x \in \gamma(\xi)$ temos que $x$ se escreve como $x=\xi(\tau)$ para algum $\tau \in \mathbb{R}$, então se $t \geq 0$ vem que $T(t) x=T(t) \xi(\tau)=\xi(t+\tau) \in \gamma(\xi)$. Reciprocamente, se $t \geq 0$ e $x \in T(t) \gamma(\xi)$, então existe $z \in \gamma(\xi)$ de maneira que $x=T(t) z$, e portanto, como $z=\xi(\tau)$ para um certo número real $\tau$, teremos $x=T(t) z=T(t) \xi(\tau)=\xi(t+\tau)$, donde $x \in \gamma(\xi)$, concluindo a invariância.

Observemos também, que toda solução global de um semigrupo $T(\cdot)$ é necessariamente contínua. Porque, dados $t_{0}$ real e $\delta>0$, fixando qualquer real $\tau \operatorname{com} \tau<t_{0}-\delta$ e usando a definição de solução, para todo real $t \in\left(t_{0}-\delta, t_{0}+\delta\right)$ podemos escrever

$$
d\left(\xi(t), \xi\left(t_{0}\right)\right)=d\left(T(t-\tau) \xi(\tau), T\left(t_{0}-\tau\right) \xi(\tau)\right)
$$

e daí a conclusão segue pela propriedade (iii) que define o conceito de semigrupo.

Os conceitos de conjunto invariante e de solução global estão conectados por meio do próximo resultado, que também explica a estrutura geométrica que têm os conjuntos invariantes em termos de órbitas globais do semigrupo.

Proposição 1.1.2. Um subconjunto $A \subset X$ é invariante por $T(\cdot)$ se, e somente se, $A$ é uma reunião de órbitas globais de $T(\cdot)$.

Demonstração: Como vimos, toda órbita global é um conjunto invariante, logo se $A$ é uma reunião de órbitas globais, então $A$ é invariante, porque a reunião de conjuntos invariantes é invariante.

Por outro lado, suponhamos que $A$ seja um conjunto invariante e tomemos um ponto qualquer $x_{0} \in A$, imediatamente da invariância resulta que $T(t) x_{0} \in A$ sempre que $t \geq 0$. Agora, ainda pela invariância, existe um ponto $x_{-1} \in A$ de forma que $x_{0}=T(1) x_{-1}$. Pelo mesmo motivo, existe $x_{-2} \in A$ tal que $x_{-1}=T(1) x_{-2}$, donde, $x_{0}=T(2) x_{-2}$. Prosseguindo analogamente, obteremos que para cada natural $n$ existe um ponto $x_{-n} \in A$ satisfazendo que $x_{-n+1}=T(1) x_{-n}$, assim $T(n) x_{-n}=x_{0}$ e mais geralmente, por um argumento de indução, vemos que $T(n) x_{-m}=x_{n-m}$ toda vez que $m \geq n$. 
Finalmente, definindo $\xi: \mathbb{R} \rightarrow X$ pondo

$$
\xi(\tau):=\left\{\begin{array}{c}
T(\tau) x_{0}, \text { se } \tau \geq 0 \\
T(\tau+n) x_{-n} \text { se } \tau \in[-n, 1-n]
\end{array},\right.
$$

vemos que $\xi$ está bem definida, no sentido de que, os valores de $\xi$ no extremo comum dos intervalos $[-n-1,-n]$ e $[-n, 1-n]$ coincidem para todo natural $n$ e, pela maneira com a qual $\xi$ foi construída, $\xi(\tau) \in A$ para todo real $\tau$. Provemos que, além disso, $\xi: \mathbb{R} \rightarrow X$ satisfaz a definição de solução global.

De fato, fixado $t \geq 0$, dado $\tau \in \mathbb{R}$, se $\tau \geq 0$ temos, em primeiro lugar, que $T(t) \xi(\tau)=T(t) T(\tau) x_{0}=T(t+\tau) x_{0}=\xi(t+\tau)$, porque $t+\tau \geq 0$.

Agora, se $\tau<0$ existe $n \in \mathbb{N}$ de maneira que $\tau \in[-n, 1-n]$ e temos dois casos a considerar:

Caso $1: t+\tau \geq 0$. Neste caso obtém-se

$$
\begin{gathered}
T(t) \xi(\tau)=T(t) T(\tau+n) x_{-n}=T([t+\tau]+n) x_{-n}= \\
T(t+\tau) T(n) x_{-n}=T(t+\tau) x_{0}=\xi(t+\tau),
\end{gathered}
$$

uma vez que $T(n) x_{-n}=x_{0}$, simplesmente pela construção da sequência $\left(x_{-n}\right)_{n \in \mathbb{N}}$.

Caso 2: $t+\tau<0$. Para tratar esta situação consideremos $m \in \mathbb{N}$ tal que $t+\tau \in$ $[-m, 1-m]$, então, claramente, $m \leq n$, donde obtém-se que

$$
\begin{gathered}
T(t) \xi(\tau)=T(t) T(\tau+n) x_{-n}=T(t+[\tau+n]) x_{-n}= \\
T([t+\tau+m]+[n-m]) x_{-n}=T(t+\tau+m) T(n-m) x_{-n} .
\end{gathered}
$$

Assim, lembrando que $x_{-m}=T(n-m) x_{-n}$, a última igualdade nos dá

$$
T(t) \xi(\tau)=T(t+\tau+m) x_{-m}=\xi(t+\tau)
$$

o que completa a demonstração.

Quando se deseja estudar o comportamento assintótico dos sistemas dinâmicos, uma ferramenta bastante útil é a semidistância de Hausdorff, a qual será a "medida" responsável por descrever a noção de proximidade entre os objetos relacionados a dinâmica do sistema.

Dados $A$ e $B$, subconjuntos não vazios de $X$, a semidistância de Hausdorff de $A$ até $B$ define-se como o número real não negativo

$$
\operatorname{dist}(A, B):=\sup _{a \in A} \inf _{b \in B} d(a, b)=\sup _{a \in A} d(a, B),
$$

onde $d(a, B):=\inf _{b \in B} d(a, b)$ indica a distância usual entre um ponto e um conjunto. 
Agora, a distância simétrica de Hausdorff entre $A$ e $B$ é definida por

$$
d_{H}(A, B):=\operatorname{dist}(A, B)+\operatorname{dist}(B, A) .
$$

Um fato que faz da semidistância de Hausdorff uma ferramenta útil nesta teoria é que ela satisfaz a desigualdade triangular, ou seja, porque vale o seguinte resultado

Lema 1.1.3. Para todos os subconjuntos não vazios $A, B$ e $C$ de $X$ vale

$$
\operatorname{dist}(A, C) \leq \operatorname{dist}(A, B)+\operatorname{dist}(B, C) .
$$

Demonstração: Com efeito, dados arbitrários $a \in A, b \in B$ e $c \in C$ tem-se $d(a, c) \leq d(a, b)+d(b, c)$, donde tomando-se o ínfimo em $C$ obtém-se

$$
d(a, C) \leq d(a, b)+d(b, C)
$$

e daí

$$
d(a, C) \leq d(a, B)+\operatorname{dist}(B, C),
$$

qualquer que seja $a \in A$. A conclusão agora segue facilmente tomando-se o supremo em $A$.

É evidente da definição que se $A \subset B$, então $\operatorname{dist}(A, B)=0$ e vale também uma espécie de recíproca, ou seja, que para quaisquer subconjuntos $A$ e $B$ de $X$ tem-se que $\operatorname{dist}(A, B)=0$ se, e somente se, $A \subset \bar{B}$. Porque, se $\operatorname{dist}(A, B)=0$ então, fixado $a \in A$ temos $d(a, B)=0$, logo, simplesmente pela definição de ínfimo, para cada natural $n$ existe um ponto $a_{n} \in B$ tal que $d\left(a, a_{n}\right)<\frac{1}{n}$, portanto $a_{n} \rightarrow a$, e isso significa que $a \in \bar{B}$.

Desta observação podemos concluir, junto com o lema anterior, que a distância simétrica de Hausdorff define, em sentido estrito, uma distância na coleção de todos os subconjuntos fechados de $X$.

Uma vez definida a semidistância de Hausdorff podemos dar sentido ao conceito de "atração".

Diz-se que um subconjunto $A$ de $X$ atrai um subconjunto $B$ de $X$, ou que o subconjunto $B$ é atraído pelo subconjunto $A$, por meio (ou ação) do semigrupo $T(\cdot)$, quando

$$
\lim _{t \rightarrow \infty} \operatorname{dist}(T(t) B, A)=0 .
$$

Recordemos que, dados um subconjunto $A \subset X$ e um número real positivo $\varepsilon$, a $\varepsilon$-vizinhança de $A$, indicada por $\mathcal{O}_{\varepsilon}(A)$, é definida como a reunião de todas as bolas abertas centradas em pontos de $A$ e possuindo raio $\varepsilon$. Simbolicamente

$$
\mathcal{O}_{\varepsilon}(A):=\bigcup_{a \in A} B(a ; \varepsilon)=\{x \in X: d(x, A)<\varepsilon\} .
$$


Da definição anterior deduz-se que um subconjunto $B$ é atraído por um subconjunto $A$ se, e somente se, para todo $\varepsilon>0$ existe $\tau=\tau(\varepsilon, B) \geq 0$ de maneira que

$$
T(t) B \subset \mathcal{O}_{\varepsilon}(A) \text { para todo } t \geq \tau \text {. }
$$

Agora podemos introduzir a noção de atrator global.

Definição 1.1.4. Um subconjunto $\mathcal{A}$ de $X$ chama-se um atrator global para o semigrupo $\{T(t): t \geq 0\}$, quando é compacto, invariante e atrai cada um dos subconjuntos limitados de $X$ pela ação de $T(\cdot)$.

Observemos que, pela maneira como definimos o conceito de atrator global, a priori, parece ser permitido que um certo semigrupo possua mais do que um atrator global. Não obstante, podemos provar facilmente que todo semigrupo possui, no máximo, um único atrator global no sentido da Definição 1.1.4.

Proposição 1.1.5. Se existe um atrator global para um semigrupo $\{T(t): t \geq 0\}$, então este atrator é único.

Demonstração: Com efeito, sejam $\mathcal{A}_{1}$ e $\mathcal{A}_{2}$ atratores globais para o semigrupo $\{T(t): t \geq 0\}$. Então, como $\mathcal{A}_{2}$ é compacto, ele é um subconjunto limitado de $X$, e assim, usando que $\mathcal{A}_{1}$ é um atrator global, teremos

$$
\lim _{t \rightarrow \infty} \operatorname{dist}\left(T(t) \mathcal{A}_{2}, \mathcal{A}_{1}\right)=0 .
$$

Mas, a invariância de $\mathcal{A}_{2}$ por $T(\cdot)$ implica $T(t) \mathcal{A}_{2}=\mathcal{A}_{2}$ qualquer que seja $t \geq 0$. Levando em conta esse fato, o limite precedente nos dá

$$
0=\lim _{t \rightarrow \infty} \operatorname{dist}\left(T(t) \mathcal{A}_{2}, \mathcal{A}_{1}\right)=\lim _{t \rightarrow \infty} \operatorname{dist}\left(\mathcal{A}_{2}, \mathcal{A}_{1}\right)=\operatorname{dist}\left(\mathcal{A}_{2}, \mathcal{A}_{1}\right),
$$

ou seja, $\operatorname{dist}\left(\mathcal{A}_{2}, \mathcal{A}_{1}\right)=0$.

Logo, como vimos antes, isto implica $\mathcal{A}_{2} \subset \overline{\mathcal{A}_{1}}=\mathcal{A}_{1}$, já que $\mathcal{A}_{1}$ é um conjunto fechado.

Invertendo os papeis de $\mathcal{A}_{1}$ e $\mathcal{A}_{2}$ no argumento anterior, conclui-se a outra inclusão, $\mathcal{A}_{1} \subset \mathcal{A}_{2}$, mostrando a igualdade entre os dois atratores.

Um dos principais problemas no estudo dos sistemas dinâmicos é descrever a estrutura geométrica que tem seu atrator global. Uma classe importante de sistemas dinâmicos autônomos para os quais se conhece muito bem a estrutura de seus atratores, são os chamados semigrupos gradientes, os quais serão estudados em detalhes neste trabalho, a partir do próximo capítulo.

Com as ferramentas que descrevemos até o momento, podemos provar dois resultados neste sentido, os quais estão contidos nas afirmações seguintes: 
Teorema 1.1.6. Se um semigrupo $\{T(t): t \geq 0\}$ em um espaço métrico $X$ possui atrator global $\mathcal{A}$, então $\mathcal{A}$ se exprime como a reunião de todos os subconjuntos invariantes limitados de $X$.

Demonstração: É evidente, do fato de que $\mathcal{A}$ é limitado e invariante, que $\mathcal{A}$ está contido na reunião de todos os subconjuntos invariantes limitados de $X$.

Reciprocamente, seja $B$ um subconjunto invariante limitado de $X$. Como $\mathcal{A}$ é o atrator global, $\mathcal{A}$ atrai $B$, isto é, $\lim _{t \rightarrow \infty} \operatorname{dist}(T(t) B, \mathcal{A})=0$. Mas, $T(t) B=B$ para todo $t \geq 0$, donde segue-se que $\operatorname{dist}(B, \mathcal{A})=0$, o que implica, como vimos, $B \subset \overline{\mathcal{A}}=\mathcal{A}$, completando a prova.

Este último resultado possui um simples corolário que é, na verdade, o primeiro resultado sobre caracterização de atratores em termos de soluções do sistema.

Corolário 1.1.7. Se um semigrupo $\{T(t): t \geq 0\}$ em um espaço métrico $X$ possui atrator global $\mathcal{A}$, então $\mathcal{A}$ é a reunião de todas as órbitas globais limitadas de $\{T(t)$ : $t \geq 0\}$.

Demonstração: Como vimos na Proposição 1.1.2, todo conjunto invariante é uma reunião de órbitas globais, assim, como $\mathcal{A}$ é um conjunto invariante, resulta que $\mathcal{A}$ é uma reunião de órbitas globais, as quais são obrigatoriamente limitadas porque $\mathcal{A}$ o é.

Por outro lado, se $\xi: \mathbb{R} \rightarrow X$ é uma solução global limitada, também vimos que sua órbita, $\gamma(\xi)$, é um subconjunto invariante limitado, logo está contido em $\mathcal{A}$ pelo teorema anterior e a conclusão segue.

O objetivo deste capítulo é desenvolver resultados gerais que nos forneçam condições suficientes para garantir a existência de atrator global, uma vez conhecido um semigrupo $\{T(t): t \geq 0\}$. Antes de começarmos com a discussão sobre tais resultados, o que será feito na próxima seção, terminemos esta apresentando mais algumas notações e definições.

Dado um subconjunto $B$ de $X$, denotemos por $\gamma^{+}(B)$ sua semiórbita positiva relativa ao semigrupo $T(\cdot)$, ou seja, o conjunto

$$
\gamma^{+}(B):=\{T(t) x: t \geq 0, x \in B\}=\bigcup_{x \in B} \gamma^{+}(x) .
$$

As vezes é útil considerar "pedaços" de órbitas para tempos arbitrariamente grandes, isto é, dados $B \subset X$ e $\tau \geq 0$ indiquemos por $\gamma_{\tau}^{+}(B)$ o conjunto $\gamma^{+}(T(\tau) B)$, ou seja,

$$
\gamma_{\tau}^{+}(B):=\{T(t) x: t \geq \tau, x \in B\}
$$


que costuma ser chamado a semiórbita positiva de $B$ à direita de $\tau$.

Diz-se que um semigrupo $\{T(t): t \geq 0\}$ é limitado, quando a semiórbita positiva de qualquer subconjunto limitado de $X$ é um limitado de $X$, enquanto diz-se que ele é eventualmente limitado, quando para cada subconjunto limitado $B \subset X$ existe $\tau=\tau(B) \geq 0$ de maneira que $\gamma_{\tau}^{+}(B)$ é um limitado de $X$.

Observemos que, se um semigrupo $\{T(t): t \geq 0\}$ em um espaço métrico $X$ possui atrator global $\mathcal{A}$, então $\{T(t): t \geq 0\}$ é necessariamente eventualmente limitado, pois dado um limitado $B \subset X$ e fazendo $\varepsilon=1$ na definição de atração teremos, em primeiro lugar, que $\mathcal{O}_{1}(\mathcal{A})$ é lmitado e, em segundo, por (1.1.3), existe $\tau=\tau(B)$ de modo que $\gamma_{\tau}^{+}(B) \subset \mathcal{O}_{1}(\mathcal{A})$, donde $\gamma_{\tau}^{+}(B)$ é limitado. Em particular, se $\xi: \mathbb{R} \rightarrow X$ é uma solução global para $T(\cdot)$ então, para todo real $\tau$, o conjunto $\{\xi(t): t \geq \tau\}$ é limitado.

Dados dois subconjuntos $B$ e $D$ de $X$, diz-se que $D$ absorve o conjunto $B$, pela ação do semigrupo, quando existe um real $\tau=\tau(B) \geq 0$ de maneira que $T(t) B \subset D$ sempre que $t \geq \tau$.

Um semigrupo $\{T(t): t \geq 0\}$ chama-se limitado dissipativo, quando existe um subconjunto limitado $D$ de $X$ que absorve cada um dos subconjuntos limitados de $X$ sob a ação de $T(\cdot)$.

Finalmente, diz-se que $\{T(t): t \geq 0\}$ é um semigrupo ponto dissipativo, quando existe um subconjunto limitado $D$ de $X$ absorvendo cada um dos pontos de $X$ pela ação de $T(\cdot)$, ou seja, quando existe um limitado $D$ tal que para todo ponto $x \in X$ existe $\tau:=\tau(x, D) \geq 0$ de modo que $T(t) x \in D$ sempre que $t \geq \tau$.

As noções de atração e absorção são equivalentes no sentido de que, um semigrupo $T(\cdot)$ é dissipativo se, e somente se, existe um subconjunto limitado $A$ que atrai cada um dos subconjuntos limitados de $X$. Com efeito, é claro da definição da semidistância de Hausdorff que se $D$ absorve cada um dos limitados de $X$, então ele atrai cada um dos

subconjuntos limitados de $X$. Reciprocamente, supondo que $A$ atrai todos os limitados então, fixando um $\varepsilon>0$ qualquer, é imediato da observação em (1.1.3) que, pondo $D:=\mathcal{O}_{\varepsilon}(A)$, resulta que $D$ é limitado e satisfaz a definição de dissipatividade, como queríamos.

\subsection{Conjuntos $\omega$-limites}

Introduziremos nesta seção o importante conceito de conjunto $\omega$-limite, que desempenhará um papel fundamental no desenvolvimento da teoria dos atratores globais, que analizaremos na próxima seção. 
Definição 1.2.1. Dado um subconjunto $B$ de $X$, seu conjunto w-limite com respeito a $T(\cdot)$, denotado por $\omega(B)$, é o conjunto

$$
\omega(B):=\bigcap_{t \geq 0}\left(\overline{\bigcup_{s \geq t} T(s) B}\right)=\bigcap_{t \geq 0} \overline{\gamma_{t}^{+}(B)} .
$$

É imediato constatar que o conjunto $\omega$-limite de qualquer subconjunto $B$ de $X$ é um conjunto fechado, simplesmente por ser uma interseção de fechados.

Do ponto de vista prático, quer dizer, na hora de se utilizar este conceito para se demonstrar teoremas, esta definição não é muito útil. Contudo, podemos substituí-la por outra mais eficiente, em termos de limites de sequências, como nos mostra a seguinte caracterização, onde pelo símbolo $\mathbb{R}_{0}^{+}$estamos indicando o conjunto dos números reais não negativos $[0, \infty)$.

Lema 1.2.2. O conjunto $\omega$-limite de um subconjunto $B \subset X$ está caracterizado por

$$
\begin{gathered}
\omega(B)=\left\{x \in X \text { : existem sequências }\left(t_{n}\right)_{n \in \mathbb{N}} \text { em } \mathbb{R}_{0}^{+} \text {com } t_{n} \rightarrow \infty\right. \text { e } \\
\left.\left(x_{n}\right)_{n \in \mathbb{N}} \text { em } B, \text { tais que } x=\lim _{n \rightarrow \infty} T\left(t_{n}\right) x_{n}\right\} .
\end{gathered}
$$

Demonstração: Com efeito, seja $\omega^{\prime}(B)$ o conjunto definido como sendo o do lado direito em (1.2.4). Por um lado, dado $x \in \omega(B)$, para cada natural $n$ temos, pela definição de $\omega(B), x \in \overline{\gamma_{n}^{+}(B)}$. Logo, para cada $n$ deve existir $z_{n} \in \gamma_{n}^{+}(B)$ tal que $d\left(x, z_{n}\right)<\frac{1}{n}$. Mas, pela definição de $\gamma_{n}^{+}(B)$, existem $t_{n} \geq n$ e $x_{n} \in B$ de maneira que $z_{n}=T\left(t_{n}\right) x_{n}$. Daí, evidentemente, vem que $x=\lim _{n \rightarrow \infty} T\left(t_{n}\right) x_{n}$, com $t_{n} \rightarrow \infty$ e $\left(x_{n}\right)_{n \in \mathbb{N}}$ em $B$, ou seja, $x \in \omega^{\prime}(B)$.

Por outro lado, seja $x \in \omega^{\prime}(B)$, então $x=\lim _{n \rightarrow \infty} T\left(t_{n}\right) x_{n}$, para certas sequências $\left(t_{n}\right)_{n \in \mathbb{N}}$ em $\mathbb{R}_{0}^{+}$, com $t_{n} \rightarrow \infty$, e $\left(x_{n}\right)_{n \in \mathbb{N}}$ em $B$.

Agora, dado $t \geq 0$ qualquer, escolhamos um natural $n(t)$ de modo que $t_{n} \geq t$ sempre que $n \geq n(t)$. Nestas condições, vemos facilmente que $T\left(t_{n}\right) x_{n} \in \gamma_{t}(B)$ toda vez que $n \geq n(t)$, donde resulta que $x \in \overline{\gamma_{t}(B)}$, e pela arbitrariedade com a qual $t \geq 0$ foi tomado, conclui-se que $x \in \bigcap_{t \geq 0} \overline{\gamma_{t}^{+}(B)}$, isto é, $x \in \omega(B)$ e o lema fica demonstrado.

Com a ajuda deste último resultado é muito simples ver que, para $B$ e $C$ subconjuntos de $X, \omega(B \cup C) \subset \omega(B) \cup \omega(C)$, se $B \subset C$ então $\omega(B) \subset \omega(C)$ e que se $\xi: \mathbb{R} \rightarrow X$ é uma solução global de $T(\cdot)$ tem-se $\omega(\xi(t))=\omega(\xi(s))$ quaisquer que sejam os números reais $s$ e $t$.

Em alguns casos, podemos tratar um conceito semelhante ao de conjunto $\omega$-limite, onde fazemos "o tempo" variar para trás, definindo o que se conhece como conjunto $\alpha$ limite. Contudo, aqui consideraremos apenas o caso de conjuntos $\alpha$-limites associados 
a uma solução global (isto é, não trabalharemos com conjuntos $\alpha$-limites associados a um subconjunto arbitrário $B$ de $X$ ). Mais precisamente

Definição 1.2.3. Seja $\xi: \mathbb{R} \rightarrow X$ uma solução global do semigrupo $\{T(t): t \geq 0\}$. Definimos seu conjunto $\alpha$-limite como:

$$
\alpha(\xi):=\left\{x \in X: \text { existe }\left(t_{n}\right)_{n \in \mathbb{N}} \text { em } \mathbb{R} \text { com } t_{n} \rightarrow-\infty \text { tal que } x=\lim _{n \rightarrow \infty} \xi\left(t_{n}\right)\right\}
$$

As principais propriedades dos conjuntos $\omega$-limites, necessárias para o estudo que faremos dos atratores globais, sempre se verificam para os semigrupos assintoticamente compactos, que a seguir definimos.

Definição 1.2.4. Um semigrupo $\{T(t): t \geq 0\}$ em um espaço métrico $X$ chamase assintoticamente compacto quando para toda sequência limitada de pontos de $X,\left(x_{n}\right)_{n \in \mathbb{N}}$, e toda sequência de números reais não negativos $\left(t_{n}\right)_{n \in \mathbb{N}} \operatorname{com} t_{n} \rightarrow \infty$, a sequência de pontos de $X,\left(T\left(t_{n}\right) x_{n}\right)_{n \in \mathbb{N}}$, possui uma subsequência convergente.

Exemplos práticos de semigrupos assintoticamente compactos, em problemas específicos, podem ser encontrados sem muita dificultade. Dentre eles, destacamos os chamados semigrupos eventualmente compatos (veja a definição abaixo) que são também eventualmente limitados.

Definição 1.2.5. Diz-se que um semigrupo $\{T(t): t \geq 0\}$ em um espaço métrico $X$ é eventualmente compacto, quando existe $t_{0}>0$ tal que a aplicação $T\left(t_{0}\right): X \rightarrow X$ é uma aplicação compacta, isto é, quando para cada subconjunto limitado $B$ de $X$ sua imagem por $T\left(t_{0}\right)$ é um conjunto relativamente compacto de $X$.

Suponhamos que $\{T(t): t \geq 0\}$ seja um semigrupo eventualmente compacto e fixemos $t_{0}>0$ de modo que $T\left(t_{0}\right): X \rightarrow X$ seja uma aplicação compacta. Então, do fato de que uma aplicação contínua transforma conjuntos compactos em conjuntos compactos e da propriedade de semigrupo, deduz-se que para todo $t \geq t_{0}, T(t): X \rightarrow$ $X$ é uma aplicação compacta, pois $T(t)=T\left(t-t_{0}\right) T\left(t_{0}\right)$ toda vez que $t \geq t_{0}$.

Para ver que um semigrupo eventualmente compacto $\{T(t): t \geq 0\}$ e eventualmente limitado é assintoticamente compacto, sejam $\left(t_{n}\right)_{n \in \mathbb{N}}$ uma sequência de números com $t_{n} \rightarrow \infty$ e $\left(x_{n}\right)_{n \in \mathbb{N}}$ uma sequência limitada de pontos de $X$. Sejam, pelo fato de que $T(\cdot)$ é eventualmente compacto, $t_{0}>0$ tal que $T\left(t_{0}\right): X \rightarrow X$ é uma aplicação compacta e, pelo fato de que $T(\cdot)$ é eventualmente limitado, $\tau \geq 0$ de maneira que a semiórbita positiva à direita de $\tau$ do subconjunto limitado $B_{0}:=\left\{x_{n}: n \in \mathbb{N}\right\}$, 
$\gamma_{\tau}^{+}\left(B_{0}\right)$, seja limitada. Finalmente, escolhamos um natural $n_{0}$ tal que $t_{n} \geq t_{0}+\tau$ para todo $n \geq n_{0}$. Definindo agora o conjunto limitado $B:=\left\{T\left(t_{n}-t_{0}\right) x_{n}: n \geq n_{0}\right\} \subset$ $\gamma_{\tau}^{+}\left(B_{0}\right)$, da compacidade eventual, resulta que $T\left(t_{0}\right) B$ é relativamente compacto e sendo $\left(T\left(t_{n}\right) x_{n}\right)_{n \geq n_{0}}$ uma sequência cujos pontos são (exatamente) os pontos de $T\left(t_{0}\right) B$, a conclusão segue.

Apresentemos agora as principais propriedades dos conjuntos $\omega$-limites para semigrupos assintoticamente compactos, as quais se encontram resumidas no seguinte lema.

Lema 1.2.6. Seja $\{T(t): t \geq 0\}$ um semigrupo assintoticamente compacto em um espaço métrico $X$. Para todo subconjunto limitado não vazio $B \subset X$, seu conjunto $\omega$-limite satisfaz as seguintes propriedades:

(i) $\omega(B)$ é não vazio, compacto, invariante e atrai $B$ pela ação de $T(\cdot)$.

(ii) $\omega(B)$ é o menor conjunto fechado de $X$ que atrai $B$.

(iii) Se $B$ é um conjunto conexo ou, mais geralmente, se existe um conexo $C$ que contém $B$ e que é atraído por $\omega(B)$, então $\omega(B)$ é conexo.

Demonstração: $\quad(i)$ Para ver que $\omega(B)$ é não vazio, escolhamos uma sequência arbitrária de números reais não negativos $\left(t_{n}\right)_{n \in \mathbb{N}} \operatorname{com} t_{n} \rightarrow \infty$ e uma sequência $\left(x_{n}\right)_{n \in \mathbb{N}}$ de pontos de $B$. Da compacidade assintótica deduz-se que a sequência $\left(T\left(t_{n}\right) x_{n}\right)_{n \in \mathbb{N}}$ possui uma subsequência convergente e o Lema 1.2.2 nos diz que este limite pertence a $\omega(B)$.

A compacidade pode ser provada da seguinte maneira: Seja $\left(x_{n}\right)_{n \in \mathbb{N}}$ uma sequência de pontos em $\omega(B)$. Graças ao Lema 1.2 .2 , a cada $n \in \mathbb{N}$ está associado um par de sequências $\left(t_{j}^{(n)}\right)_{j \in \mathbb{N}}$ em $\mathbb{R}_{0}^{+}$, com $t_{j}^{(n)} \underset{j \rightarrow \infty}{\rightarrow} \infty$, e $\left(x_{j}^{(n)}\right)_{j \in \mathbb{N}}$ em $B$ tais que $x_{n}=$ $\lim _{j \rightarrow \infty} T\left(t_{j}^{(n)}\right) x_{j}^{(n)}$, donde concluimos que para cada natural $n$ existe um natural $j_{n}$ de meneira que

$$
d\left(x_{n}, T\left(t_{j_{n}}^{(n)}\right) x_{j_{n}}^{(n)}\right)<\frac{1}{n} \operatorname{com} t_{j_{n}}^{(n)} \geq n .
$$

Como $t_{j_{n}}^{(n)} \underset{n \rightarrow \infty}{\rightarrow} \infty$, pela compacidade assintótica, podemos extrair uma subsequência convergente de $\left(T\left(t_{j_{n}}^{(n)}\right) x_{j_{n}}^{(n)}\right)_{n \in \mathbb{N}}$, a qual denotaremos por

$$
\left(T\left(t_{j_{n_{k}}}^{\left(n_{k}\right)}\right) x_{j_{n_{k}}}^{\left(n_{k}\right)}\right)_{k \in \mathbb{N}}
$$

e seu limite por $x$. O Lema 1.2.2 implica agora que $x \in \omega(B)$, e de (1.2.5) obtém-se

$$
d\left(x_{n_{k}}, T\left(t_{j_{n_{k}}}^{\left(n_{k}\right)}\right) x_{j_{n_{k}}}^{\left(n_{k}\right)}\right)<\frac{1}{n_{k}},
$$

donde resulta, após tomar o limite para $k \rightarrow \infty$, que $x_{n_{k}} \underset{k \rightarrow \infty}{\rightarrow} x$, o que demonstra a compacidade de $\omega(B)$. 
Provemos agora que $\omega(B)$ é invariante por $T(\cdot)$. Em primeiro lugar, sejam $x \in \omega(B)$, $\left(t_{n}\right)_{n \in \mathbb{N}}$ em $\mathbb{R}_{0}^{+}$, com $t_{n} \rightarrow \infty$, e $\left(x_{n}\right)_{n \in \mathbb{N}}$ em $B$ de maneira que $x=\lim _{n \rightarrow \infty} T\left(t_{n}\right) x_{n}$. Então, se $t \geq 0$ é dado, da continuidade do operador $T(t): X \rightarrow X$ e da propriedade de semigrupo segue-se que

$$
T(t) x=T(t)\left(\lim _{n \rightarrow \infty} T\left(t_{n}\right) x_{n}\right)=\lim _{n \rightarrow \infty} T\left(t+t_{n}\right) x_{n},
$$

com $t+t_{n} \rightarrow \infty$ e $\left(x_{n}\right)_{n \in \mathbb{N}}$ em $B$, o que significa que $T(t) x \in \omega(B)$ e estabelece a inclusão $T(t) \omega(B) \subset \omega(B)$. Reciprocamente, sejam $x \in \omega(B),\left(t_{n}\right)_{n \in \mathbb{N}}$ em $\mathbb{R}_{0}^{+}$, com $t_{n} \rightarrow \infty$, e $\left(x_{n}\right)_{n \in \mathbb{N}}$ em $B$ de maneira que $x=\lim _{n \rightarrow \infty} T\left(t_{n}\right) x_{n}$. Fixando $t \geq 0$, vemos que

$$
x=\lim _{n \rightarrow \infty} T\left(t_{n}\right) x_{n}=\lim _{n \rightarrow \infty} T\left(t+\left(t_{n}-t\right)\right) x_{n}=\lim _{n \rightarrow \infty} T(t) T\left(t_{n}-t\right) x_{n} .
$$

Por outro lado, usando novamente a compacidade assintótica, obtém-se um ponto $z \in$ $X$ e uma subsequência $\left(T\left(t_{n_{j}}-t\right) x_{n_{j}}\right)_{j \in \mathbb{N}}$ de $\left(T\left(t_{n}-t\right) x_{n}\right)_{n \in \mathbb{N}}$ de modo que

$$
z=\lim _{j \rightarrow \infty} T\left(t_{n_{j}}-t\right) x_{n_{j}}
$$

donde segue-se, graças ao Lema 1.2.2, que $z \in \omega(B)$, e usando a continuidade de $T(t)$ : $X \rightarrow X$ em (1.2.6) e o fato de que toda subsequência de uma sequência convergente é convergente e converge ao mesmo limite, obtém-se

$$
x=T(t)\left(\lim _{j \rightarrow \infty} T\left(t_{n_{j}}-t\right) x_{n_{j}}\right)=T(t) z,
$$

mostrando que $x \in T(t) \omega(B)$, concluindo a inclusão $T(t) \omega(B) \supset \omega(B)$ e, portanto, a invariância de $\omega(B)$.

Vejamos agora que $\omega(B)$ atrai $B$ pela ação de $T(\cdot)$, ou seja, que $\lim _{t \rightarrow \infty} \operatorname{dist}(T(t) B, \omega(B))=$ 0 . Suponhamos que isto não seja certo, então deve existir $\varepsilon>0$ e podemos construir uma sequência $\left(t_{n}\right)_{n \in \mathbb{N}}$ de números reais positivos com $t_{n} \rightarrow \infty$ tais que

$$
\operatorname{dist}\left(T\left(t_{n}\right) B, \omega(B)\right)>\varepsilon \text { para todo } n \in \mathbb{N} \text {. }
$$

Por outro lado, a definição da semidistância nos diz que para cada natural $n$ podemos encontrar um ponto $x_{n} \in B$ tal que

$$
d\left(T\left(t_{n}\right) x_{n}, \omega(B)\right)>\varepsilon,
$$

mas, a compacidade assintótica de $T(\cdot)$ nos permite extrair uma subsequência de $\left(T\left(t_{n}\right) x_{n}\right)_{n \in \mathbb{N}}$ que converge a um ponto $x$ que, obrigatoriamente, está em $\omega(B)$. Então, a continuidade da função distância de um ponto a um conjunto e (1.2.7) nos dizem que $d(x, \omega(B)) \geq \varepsilon$, contradizendo que $x \in \omega(B)$, estabelecendo que $\omega(B)$ atrai $B$ e completando a prova do item $(i)$. 
Provemos $(i i)$ : Da definição de $\omega(B)$, segue-se que o mesmo é fechado e, pelo item anterior, atrai $B$ por $T(\cdot)$. Para provar que é, precisamente, o menor fechado com esta propiedade, consideremos $F$ um subconjunto fechado de $X$ que atrai $B$ por $T(\cdot)$ e mostremos que $\omega(B) \subset F$.

Com efeito, caso contrário, existiria um ponto $x \in \omega(B) \operatorname{com} x \notin F$. Como $F$ é um conjunto fechado, tem-se $d(x, F)=: \delta>0$. Agora, sejam $\left(t_{n}\right)_{n \in \mathbb{N}}$ em $\mathbb{R}_{0}^{+}$, com $t_{n} \rightarrow \infty$, e $\left(x_{n}\right)_{n \in \mathbb{N}}$ em $B$ tais que $x=\lim _{n \rightarrow \infty} T\left(t_{n}\right) x_{n}$.

Por outro lado, como $F$ atrai $B$, podemos encontrar $t^{*}>0$ de modo que

$$
\operatorname{dist}(T(t) B, F)<\frac{\delta}{2} \text { sempre que } t \geq t^{*} .
$$

Logo

$$
d(T(t) z, F)<\frac{\delta}{2} \text { para todo } z \in B \text { e todo } t \geq t^{*} .
$$

Mas, escolhendo $n\left(t^{*}\right) \in \mathbb{N}$ tal que $t_{n} \geq t^{*}$ sempre que $n \geq n\left(t^{*}\right)$, conclui-se que para todo $n \geq n\left(t^{*}\right)$ vale

$$
d\left(T\left(t_{n}\right) x_{n}, F\right)<\frac{\delta}{2}
$$

donde, pela continuidade da função $X \ni w \longmapsto d(w, F) \in \mathbb{R}$, após passarmos o limite em $n$, deduz-se que $d(x, F) \leq \frac{\delta}{2}$, o que contraria a escolha de $x$ e demonstra o item $(i i)$.

Para provar ( iii) suponhamos que exista um conjunto conexo $C$ contendo $B$ e que seja atraído por $\omega(B)$, mas que $\omega(B)$ não seja conexo. Então, podemos escrever $\omega(B)$ como reunião de dois conjuntos fechados $(\mathrm{em} \omega(B))$ disjuntos e não vazios $F_{1}$ e $F_{2}$. Segue-se do item $(i)$ que $F_{1}$ e $F_{2}$ são compactos, logo $d\left(F_{1}, F_{2}\right)=: \delta>0$, onde

$$
d\left(F_{1}, F_{2}\right):=\inf \left\{d(a, b): a \in F_{1}, b \in F_{2}\right\}
$$

Agora, como $\omega(B)$ atrai $C$, existe $t^{*}>0$ tal que

$$
T(t) C \subset \mathcal{O}_{\frac{\delta}{2}}(\omega(B)) \text { para todo } t \geq t^{*}
$$

portanto

$$
\gamma_{t^{*}}^{+}(C) \subset \mathcal{O}_{\frac{\delta}{2}}(\omega(B))=\mathcal{O}_{\frac{\delta}{2}}\left(F_{1}\right) \cup \mathcal{O}_{\frac{\delta}{2}}\left(F_{2}\right) .
$$

Mas $\gamma_{t^{*}}^{+}(C)$ é a imagem do conjunto conexo $\left[t^{*}, \infty\right) \times C$ pela aplicação contínua $[0, \infty) \times X \ni(t, x) \longmapsto T(t) x \in X$ e, por isso, $\gamma_{t^{*}}^{+}(C)$ é um conjunto conexo. Como $\mathcal{O}_{\frac{\delta}{2}}\left(F_{1}\right)$ e $\mathcal{O}_{\frac{\delta}{2}}\left(F_{2}\right)$ são abertos disjuntos, $\gamma_{t^{*}}^{+}(C)$ deve estar inteiramente contido em exatamente um destes dois conjuntos. Suponhamos, para fixar idéias, que seja $\gamma_{t^{*}}^{+}(C) \subset$ $\mathcal{O}_{\frac{\delta}{2}}\left(F_{1}\right)$. Então,

$$
F_{2} \subset \omega(B) \subset \overline{\gamma_{t^{*}}^{+}(B)} \subset \overline{\gamma_{t^{*}}^{+}(C)} \subset \overline{\mathcal{O}_{\frac{\delta}{2}}\left(F_{1}\right)},
$$


dizendo que $d\left(F_{2}, F_{1}\right) \leq \frac{\delta}{2}$. O que contradiz a definição de $\delta$ e permite concluir que $\omega(B)$ é um conjunto conexo quando $\omega(B)$ atrai um conexo que contém $B$.

O caso em que $B$ é conexo, segue do que acabamos de provar acima simplesmente fazendo $C=B$ e usando o item $(i)$, terminando a demonstração do lema.

Para os conjuntos $\alpha$-limites das soluções globais limitadas dos semigrupos assintoticamente compactos podem ser demonstradas, com provas inteiramente análogas, propriedades similares às apresentadas no lema anterior, mais precisamente, tem-se o seguinte resultado:

Lema 1.2.7. Seja $\xi: \mathbb{R} \rightarrow X$ uma solução global limitada para um semigrupo assintoticamente compacto $\{T(t): t \geq 0\}$ em um espaço métrico $X$. Então, o conjunto $\alpha$-limite de $\xi, \alpha(\xi)$, é não vazio, compacto, invariante, conexo e

$$
\lim _{t \rightarrow-\infty} d(\xi(t), \alpha(\xi))=0
$$

Demonstração: Provemos apenas que $\alpha(\xi)$ é não vazio e que vale a convergência em (1.2.8), porque as demais propriedades se demonstram analogamente ao caso do conjunto $\omega$-limite.

Com efeito, seja $\left(t_{n}\right)_{n \in \mathbb{N}}$ uma sequência arbitrária de números não positivos com $t_{n} \rightarrow-\infty$. Escrevendo para cada natural $n$

$$
\xi\left(t_{n}\right)=T\left(-t_{n}\right) \xi\left(2 t_{n}\right)
$$

como $-t_{n} \rightarrow \infty$, e $\left(\xi\left(2 t_{n}\right)\right)_{n \in \mathbb{N}}$ é uma sequência limitada de pontos de $X$, a compacidade assintótica de $T(\cdot)$ assegura que $\left(T\left(-t_{n}\right) \xi\left(2 t_{n}\right)\right)_{n \in \mathbb{N}}$ possui uma subsequência convergente e, portanto, $\left(\xi\left(t_{n}\right)\right)_{n \in \mathbb{N}}$ possui uma subsequência convergente então, por definição, o limite de uma tal subsequência deve pertencer a $\alpha(\xi)$, provando que $\alpha(\xi) \neq \varnothing$.

Por outro lado, suponhamos que não se tenha a atração afirmada em (1.2.8). Então, existem $\varepsilon>0$ e uma sequência $\left(t_{n}\right)_{n \in \mathbb{N}}$ de números não positivos com $t_{n} \rightarrow-\infty$ de modo que

$$
d\left(\xi\left(t_{n}\right), \alpha(\xi)\right) \geq \varepsilon \text { para todo } n \in \mathbb{N} \text {. }
$$

Mas, repetindo o argumento que usamos para provar que $\alpha(\xi) \neq \varnothing$ conclui-se que a sequência $\left(\xi\left(t_{n}\right)\right)_{n \in \mathbb{N}}$ possui uma subsequência convergente para um ponto $x \in X$, e este ponto deverá pertencer a $\alpha(\xi)$, o que contradiz (1.2.9) e, portanto, (1.2.8) é correto.

Terminemos esta seção provando um resultado bastante útil, relacionado aos conjuntos $\omega$-limites, que utilizaremos em diversas ocasiões no decorrer deste texto. 
Lema 1.2.8. Sejam $\{T(t): t \geq 0\}$ um semigrupo em um espaço métrico $X$ e $A \subset X$ um conjunto fechado e invariante. Então

$$
\omega(A)=A
$$

Demonstração: Com efeito, por um lado, seja $x \in A$, então, pela invariância de $A$, resulta que para cada natural $n$ existe um ponto $x_{n} \in A$ tal que $x=T(n) x_{n}$ e, evidentemente, $x=\lim _{n \rightarrow \infty} T(n) x_{n}$, $\log 0 x \in \omega(A)$ em virtude do Lema 1.2.2, estabelecendo a inclusão $A \subset \omega(A)$.

Por outro, sejam $x \in \omega(A),\left(t_{n}\right)_{n \in \mathbb{N}}$ em $\mathbb{R}_{0}^{+}$e $\left(x_{n}\right)_{n \in \mathbb{N}}$ em $A$ tais que $x=\lim _{n \rightarrow \infty} T\left(t_{n}\right) x_{n}$. Usando a invariância de $A$ vemos que $T\left(t_{n}\right) x_{n} \in A$ para todo natural $n$, donde $x \in$ $\bar{A}=A$, porque $A$ é fechado, dizendo que $\omega(A) \subset A$ e a prova está terminada.

Observemos que a primeira parte da demonstração acima, estabelece que para todo conjunto invariante $A$ (não necessariamente fechado) tem-se $A \subset \omega(A)$.

\subsection{Existência de atratores}

Analizemos agora o problema de se estabelecer condições suficientes que assegurem a existência de atrator global para um dado semigrupo $\{T(t): t \geq 0\}$ em um espaço métrico $X=(X, d)$. Provamos aqui, que a existência de atrator global para um sistema autônomo é um fenômeno equivalente à compacidade assintótica com dissipatividade, o que é o conteúdo do seguinte resultado:

Teorema 1.3.1. Seja $\{T(t): t \geq 0\}$ um semigrupo em um espaço métrico $X$. Então, $\{T(t): t \geq 0\}$ possui atrator global $\mathcal{A}$ se, e somente se, é assintoticamente compacto e limitado dissipativo. Além disso, em caso afirmativo, se $\mathcal{B}$ denota a coleção de todos os subconjuntos limitados não vazios de $X$, então o atrator $\mathcal{A}$ vem dado por

$$
\mathcal{A}=\bigcup_{B \in \mathcal{B}} \omega(B)
$$

Demonstração: Suponhamos primeiramente que exista o atrator global $\mathcal{A}$ para $\{T(t): t \geq 0\}$. Então, como vimos no final da Seção 1.1, necessariamente $\{T(t): t \geq 0\}$ é dissipativo. Para ver que é também assintoticamente compacto, sejam $\left(x_{n}\right)_{n \in \mathbb{N}}$ uma sequência limitada de pontos de $X$ e $\left(t_{n}\right)_{n \in \mathbb{N}}$ uma sequência de números não negativos $\operatorname{com} t_{n} \rightarrow \infty$.

Por um lado, considerando o conjunto limitado $B:=\left\{x_{n}: n \in \mathbb{N}\right\}$ tem-se

$$
\lim _{t \rightarrow \infty} \operatorname{dist}(T(t) B, \mathcal{A})=0,
$$


em particular,

$$
\lim _{n \rightarrow \infty} \sup _{x^{\prime} \in B} d\left(T\left(t_{n}\right) x^{\prime}, \mathcal{A}\right)=\lim _{n \rightarrow \infty} \operatorname{dist}\left(T\left(t_{n}\right) B, \mathcal{A}\right)=0
$$

Então, simplesmente aplicando a definição de limite de sequências em (1.3.11), para cada $j \in \mathbb{N}$ podemos encontrar um ponto $z_{j} \in \mathcal{A}$ de maneira que

$$
d\left(T\left(t_{n_{j}}\right) x_{n_{j}}, z_{j}\right)<\frac{1}{j}
$$

Agora, como $\mathcal{A}$ é um conjunto compacto, a sequência $\left(z_{j}\right)_{j \in \mathbb{N}}$ possui uma subsequência convergente. Denotando tal subsequência da mesma maneira, seja $x \in \mathcal{A}$ seu limite. Então, por (1.3.12),

$$
d\left(T\left(t_{n_{j}}\right) x_{n_{j}}, x\right) \leq d\left(T\left(t_{n_{j}}\right) x_{n_{j}}, z_{j}\right)+d\left(z_{j}, x\right)<\frac{1}{j}+d\left(z_{j}, x\right),
$$

donde $T\left(t_{n_{j}}\right) x_{n_{j}} \underset{j \rightarrow \infty}{\rightarrow} x$, provando a compacidade assintótica de $\{T(t): t \geq 0\}$.

Reciprocamente, suponhamos que $\{T(t): t \geq 0\}$ é assintoticamente compacto e limitado dissipativo. Seja $\mathcal{A}:=\bigcup_{B \in \mathcal{B}} \omega(B)$ o conjunto definido em (1.3.10) e provemos que $\mathcal{A}$ é, de fato, o atrator global de $\{T(t): t \geq 0\}$.

Em primeiro lugar, notemos que, graças ao Lema 1.2.6, para cada $B \in \mathcal{B}$, o conjunto $\omega(B)$ é não vazio, compacto e invariante atraindo $B$ pela ação de $T(\cdot)$. Daí, vemos que $\mathcal{A}$ é invariante, por ser uma reunião de conjuntos invariantes e, além disso, atrai cada um dos limitados de $X$ por meio de $T(\cdot)$. Logo o teorema resultará demonstrado se provarmos que $\mathcal{A}$ é um conjunto compacto, o que faremos usando a dissipatividade de $\{T(t): t \geq 0\}$

Com efeito, seja $D \subset X$ um subconjunto limitado que absorve todos os subconjuntos limitados de $X$ por meio de $T(\cdot)$. Tomando o fecho de $D$, caso seja necessário, podemos supor que $D$ é fechado, então sua propriedade de absorção junto com a propriedade (ii) dada no Lema 1.2.6, nos dizem que $\omega(B) \subset D$ para todo $B \in \mathcal{B}$. Assim $\mathcal{A} \subset D$ e portanto $\omega(\mathcal{A}) \subset \omega(D)$. Como $\mathcal{A}$ é invariante temos $\mathcal{A} \subset \omega(\mathcal{A})$ e daí conclui-se a cadeia de inclusões

$$
\mathcal{A} \subset \omega(\mathcal{A}) \subset \omega(D) \subset \mathcal{A},
$$

sendo a última delas devida ao fato de que $D \in \mathcal{B}$. Consequentemente $\omega(D)=\mathcal{A}$, concluindo a compacidade de $\mathcal{A}$, levando em conta o Lema 1.2.6.

Tem-se também o resultado a seguir, que é bastante útil quando se deseja demonstrar que um semigrupo gradiente possui atrator global (confira o Teorema 2.3.1 no próximo capítulo). 
Corolário 1.3.2. Seja $\{T(t): t \geq 0\}$ um semigrupo em um espaço métrico $X$. Se $T(\cdot)$ é eventualmente compacto, eventualmente limitado e ponto dissipativo, então $T(\cdot)$ possui atrator global.

Demonstração: Como $T(\cdot)$ é eventualmente compacto e eventualmente limitado, ele é assintoticamente compacto, assim, em virtude do teorema anterior, é suficiente provarmos que $T(\cdot)$ é também limitado dissipativo.

Com efeito, seja $D_{0}$ um subconjunto limitado de $X$ que absorve cada um dos pontos de $X$ pela ação de $T(\cdot)$ e consideremos o conjunto limitado $D_{1}:=\mathcal{O}_{1}\left(D_{0}\right)$. Sendo $T(\cdot)$ um semigrupo eventualmente limitado, existe $\tau^{*} \geq 0$ de modo que o conjunto

$$
D:=\gamma_{\tau^{*}}^{+}\left(D_{1}\right)
$$

é limitado.

Provemos que $D$ absorve cada um dos subconjuntos limitados $X$.

Em primeiro lugar, observemos que $D$ absorve subconjuntos compactos de $X$ pela ação $T(\cdot)$. De fato, dado $K \subset X$ um subconjunto compacto, para cada $x \in K$ seja $\tau_{x} \geq 0$ tal que

$$
T(t) x \in D_{1} \text { para todo } t \geq \tau_{x}
$$

Como $D_{1}$ é um conjunto aberto, para cada $x \in K$, pela continuidade do operador $T\left(\tau_{x}\right): X \rightarrow X$, existe $\delta_{x}>0$ tal que

$$
T\left(\tau_{x}\right) \mathcal{O}_{\delta_{x}}(x) \subset D_{1}
$$

Daí, a definição do conjunto $D$ nos mostra que

$$
T(t) \mathcal{O}_{\delta_{x}}(x) \subset D \text { para todo } t \geq \tau_{x}+\tau^{*}
$$

Pela compacidade de $K$, sejam $x_{1}, x_{2}, \cdots, x_{n}$, um número finito de pontos de $K$, de modo que pondo, para $j=1,2, \cdots, n$, por simplicidade, $\delta_{j}:=\delta_{x_{j}}$ e $\tau_{j}=\tau_{x_{j}}$, tem-se

$$
K \subset \bigcup_{j=1}^{n} \mathcal{O}_{\delta_{j}}\left(x_{j}\right)
$$

Usando este fato junto com (1.3.14) vemos que se $\tau_{K}:=\max _{1 \leq j \leq p} \tau_{j}$ então

$$
T(t) K \subset D \text { para todo } t \geq \tau^{*}+\tau_{K}
$$

Finalmente, pelo fato de que $T(\cdot)$ é eventualmente compacto, existe $t_{0}>0$ de modo que $T\left(t_{0}\right): X \rightarrow X$ é uma aplicação compacta. Então, dado um subconjunto limitado 
qualquer $B \subset X$ o conjunto $K:=\overline{T\left(t_{0}\right) B}$ é um compacto que contém $T\left(t_{0}\right) B$ e então (1.3.15) nos diz que

$$
T(t) T\left(t_{0}\right) B \subset T(t) K \subset D \text { para todo } t \geq \tau^{*}+\tau_{K} .
$$

Logo, pondo $\tau(B):=t_{0}+\tau_{K}+\tau^{*}$, conclui-se que

$$
T(t) B \subset D \text { para todo } t \geq \tau(B),
$$

demonstrando que $D$ é um limitado que absove cada um dos subconjuntos limitados de $X$ pela ação de $T(\cdot)$, e consequentemente, $T(\cdot)$ é limitado dissipativo, completando a demonstração. 


\section{Capítulo 2 Semigrupos Gradientes}

Apresentaremos agora a classe dos semigrupos gradientes. Nosso principal resultado, no caso autônomo, consiste em caracterizar estes sistemas por meio da dinâmica que possuem.

Neste capítulo, veremos que os semigrupos gradientes possuem uma dinâmica que pode ser compreendida de forma bastante detalhada. Mostraremos que sua dinâmica fica completamente determinada por meio de uma função auxiliar, chamada função de Lyapunov, possuindo propriedades bastante particulares, evidenciando, assim, que os sistemas gradientes compõem uma classe bastante nobre de semigrupos.

\subsection{Função de Lyapunov}

A fim de introduzir o conceito de função de Lyapunov, precisaremos definir mais algumas noções; uma delas é a de conjunto invariante isolado.

Definição 2.1.1. Seja $\{T(t): t \geq 0\}$ um semigrupo em um espaço métrico $X$.

(i) Diz-se que um conjunto invariante $\Xi \subset X$ é um conjunto invariante isolado, quando existe $\delta>0$ tal que $\Xi$ é o conjunto invariante maximal de $T(\cdot)$ contido em $\mathcal{O}_{\delta}(\Xi)$, em outros palavras, se $A$ é um conjunto invariante por $T(\cdot)$ contido em $\mathcal{O}_{\delta}(\Xi)$, então $A \subset \Xi$.

(ii) Seja $\boldsymbol{\Xi}:=\left\{\Xi_{1}, \Xi_{2}, \cdots, \Xi_{n}\right\}$ um conjunto finito de conjuntos invariantes por $T(\cdot)$. Diz-se que $\boldsymbol{\Xi}$ é uma família (finita) disjunta de conjuntos invariantes isolados, quando cada um de seus elementos é um conjunto invariante isolado, segundo $(i)$, e existe $\varepsilon>0$ de maneira que $\mathcal{O}_{\varepsilon}\left(\Xi_{i}\right) \cap \mathcal{O}_{\varepsilon}\left(\Xi_{j}\right)=\varnothing$ sempre que $1 \leq i<j \leq n$. 
Não é difícil ver que, se $T(\cdot)$ possui atrator global, então o fecho de cada um de seus subconjuntos invariantes limitados é também invariante, de onde resulta que para estes semigrupos, seus invariantes isolados limitados são conjuntos fechados.

Outro conceito fundamental relacionado ao anterior é o de ponto de equilíbrio. Os pontos de equilíbrio e, mais geralmente, os conjuntos invariantes isolados, são objetos responsáveis pela organização da dinâmica do sistema.

Definição 2.1.2. Uma solução global $\xi: \mathbb{R} \rightarrow X$ chama-se uma solução estacionária ou um ponto (ou solução) de equlíbrio de $\{T(t): t \geq 0\}$, quando é uma aplicação constante, ou seja, quando é da forma $\xi(t)=z^{*}$ para todo real $t$ e um certo ponto $z^{*} \in X$. Indicaremos por $\mathcal{E}$ o conjunto dos pontos de equilibrio de $T(\cdot)$.

Se $z^{*}$ é um ponto de equilíbrio de $T(\cdot)$, então $T(t) z^{*}=z^{*}$ qualquer que seja $t \geq 0$.

Sejam $I \subset \mathbb{R}$ um conjunto de números reais e $\varphi: I \rightarrow \mathbb{R}$ uma função real definida em $I$, recordemos:

Diz-se que $\varphi$ é decrescente (resp. crescente) quando dados $t<s$ em $I$ tem-se $\varphi(t)>\varphi(s)($ resp. $\varphi(t)<\varphi(s))$.

Diz-se que $\varphi$ é não crescente (resp. não decrescente) quando dados $t<s$ em $I$ tem-se $\varphi(t) \geq \varphi(s)($ resp. $\varphi(t) \leq \varphi(s))$.

Já temos tudo o que precisamos para definir os semigrupos gradientes.

Definição 2.1.3. Sejam $\{T(t): t \geq 0\}$ um semigrupo em um espaço métrico $X e$ $\Xi:=\left\{\Xi_{1}, \Xi_{2}, \cdots, \Xi_{n}\right\}$ uma família finita disjunta de conjuntos invariantes isolados. Diz-se que $\{T(t): t \geq 0\}$ é um semigrupo gradiente generalizado com respeito $\grave{a}$ família $\Xi$, quando existe uma função $V: X \rightarrow \mathbb{R}$ satisfazendo as seguintes quatro propriedades:

(i) $V: X \rightarrow \mathbb{R}$ é uma função contínua.

(ii) $V: X \rightarrow \mathbb{R}$ é não crescente ao longo de soluções, isto é, para todo $x \in X$ a função real de uma variável real $[0, \infty) \ni t \longmapsto V(T(t) x) \in \mathbb{R}$ é não crescente.

(iii) Se para algum $x \in X$ tem-se $V(T(t) x)=V(x)$ para todo $t \geq 0$, então $x \in \Xi$ para algum $\Xi \in \Xi$.

(iv) $V: X \rightarrow \mathbb{R}$ é constante sobre cada subconjunto invariante isolado pertencente a $\Xi$.

Uma função $V: X \rightarrow \mathbb{R}$ satisfazendo estas condições chama-se uma função de Lyapunov generalizada para $T(\cdot)$ com respeito à família $\Xi$. 
No caso especial em que se tem $\boldsymbol{\Xi}=\mathcal{E}:=\left\{z_{1}^{*}, z_{2}^{*} \cdots, z_{n}^{*}\right\}$, diz-se simplesmente que $T(\cdot)$ é um semigrupo gradiente e a função $V: X \rightarrow \mathbb{R}$ associada, uma função de Lyapunov para $T(\cdot)$.

Observemos que um semigrupo gradiente pode ser entendido como uma terna $(T(\cdot), \boldsymbol{\Xi}, V)$, onde $T(\cdot)$ representa o semigrupo, $\boldsymbol{\Xi}$ a família finita disjunta de conjuntos invariantes isolados e $V$ a função de Lyapunov correspondente.

Podemos ilustrar a definição anterior de função de Lyapunov com o seguinte exemplo, o qual é o protótipo dos sistemas gradientes que costumam ser encontrados nas aplicações. Sua leitura, contudo, pode ser omitida sem nenhum prejuizo ao entendimento do restante do texto.

Exemplo 2.1.4. Sejam $N \in \mathbb{N}$ e $f$ uma função $C^{2}\left(\mathbb{R}^{N} ; \mathbb{R}\right)$ tal que para alguma constante $C>0$ tem-se

$$
|\nabla f(x)| \leq C(1+|x|) \text { qualquer que seja } x \in \mathbb{R}^{N} .
$$

Consideremos o problema de Cauchy

$$
\left\{\begin{array}{c}
\dot{x}=-\nabla f(x), t>0 \\
x(0)=x_{0} \in \mathbb{R}^{N}
\end{array}\right.
$$

onde, evidentemente, $\nabla f(x)$ representa o gradiente da função $f$ avaliado no ponto $x \in \mathbb{R}^{N}$.

Sendo $\nabla f: \mathbb{R}^{N} \rightarrow \mathbb{R}^{N}$ um campo de vetores de classe $C^{1}$, portanto localmente Lipschitz, e levando em conta (2.1.1), sabemos que o operador solução associado ao problema (2.1.2) define um semigrupo (de fato um grupo) no espaço métrico $\mathbb{R}^{N}$. Em outras palavras, definindo para $t \geq 0$ e $x_{0} \in \mathbb{R}^{N} T(t) x_{0}:=x\left(t, x_{0}\right)$, onde $x\left(\cdot, x_{0}\right)$ : $[0, \infty) \rightarrow \mathbb{R}^{N}$ é a única solução (em sentido clássico) do problema (2.1.2), resulta que $\{T(t): t \geq 0\}$ é um semigrupo em $\mathbb{R}^{N}$. Suponhamos que $\nabla f: \mathbb{R}^{N} \rightarrow \mathbb{R}^{N}$ possua somente um número finito de zeros em $\mathbb{R}^{N}$, digamos, $\mathcal{E}:=\left\{z_{1}^{*}, z_{2}^{*}, \cdots, z_{n}^{*}\right\}$.

Nestas condições, a função $f: \mathbb{R}^{N} \rightarrow \mathbb{R}$ é uma função de Lyapunov para o semigrupo $\{T(t): t \geq 0\}$ com respeito ao conjunto $\mathcal{E}$.

Demonstração: Evidentemente, $f: \mathbb{R}^{N} \rightarrow \mathbb{R}$ é uma função contínua, já que é de classe $C^{2}$, e $\mathcal{E}=\left\{z_{1}^{*}, z_{2}^{*}, \cdots, z_{n}^{*}\right\}$ é o conjunto de todos os pontos de equilíbrio de $\{T(t): t \geq 0\}$. Logo, também verifica-se a propriedade (iv) da Definição 2.1.3. Portanto, resta apenas provar as propriedades $(i i)$ e (iii) da definição de função de Lyapunov. 
Com efeito, dado $x_{0} \in \mathbb{R}^{N}$, a função real de uma variável real $[0, \infty) \ni t \longmapsto$ $f\left(T(t) x_{0}\right)=f\left(x\left(t, x_{0}\right)\right) \in \mathbb{R}$ é de classe $C^{1}$ e então, a regra da cadeia e o fato de que $x\left(\cdot, x_{0}\right):[0, \infty) \rightarrow \mathbb{R}^{N}$ é solução do problema (2.1.2), nos dizem que, para todo $t>0$

$$
\frac{d}{d t}\left(f \circ x\left(\cdot, x_{0}\right)\right)(t)=\nabla f\left(x\left(t, x_{0}\right)\right) \cdot \dot{x}\left(t, x_{0}\right)=-\left|\nabla f\left(x\left(t, x_{0}\right)\right)\right|^{2} \leq 0,
$$

onde o ponto "." representa o produto escalar euclidiano em $\mathbb{R}^{N}$ e $|\cdot|$ sua norma correspondente.

A igualdade (2.1.3) mostra que a derivada da função

$$
[0, \infty) \ni t \longmapsto f\left(T(t) x_{0}\right)=f\left(x\left(t, x_{0}\right)\right) \in \mathbb{R}
$$

é não positiva, logo tal função deve ser não crescente, ficando estabelecida a condição (ii).

Finalmente, suponhamos que $x_{0} \in \mathbb{R}^{N}$ seja tal que $f\left(T(t) x_{0}\right)=f\left(x_{0}\right)$ para todo $t \geq 0$. Então (2.1.3) nos mostra que para todo $t>0$ temos

$$
0=\frac{d}{d t} f\left(x_{0}\right)=\frac{d}{d t}\left(f \circ x\left(\cdot, x_{0}\right)\right)(t)=-\left|\nabla f\left(x\left(t, x_{0}\right)\right)\right|^{2}=-\left|\dot{x}\left(t, x_{0}\right)\right|^{2},
$$

ou seja, $\dot{x}\left(t, x_{0}\right)=0$ para todo $t>0$, donde $x\left(t, x_{0}\right)=x_{0}$ para todo $t>0$, portanto $x_{0} \in \mathcal{E}$, completando a justificativa do exemplo.

\subsection{Estrutura dos semigrupos gradientes}

Os semigrupos gradientes constituem um dos poucos exemplos de sistemas, que se conhecem, para os quais é possível descrever, de maneira bastante precisa, a dinâmica que possuem. Comecemos o estudo do comportamento das soluções globais de um sistema desta natureza sendo necessário, para isto, introduzir a noção de estrutura homoclínica como segue:

Definição 2.2.1. Seja $\{T(t): t \geq 0\}$ um semigrupo em um espaço métrico $X$ possuindo atrator global $\mathcal{A}$ e uma familia finita disjunta de conjuntos invariantes isolados limitados $\boldsymbol{\Xi}:=\left\{\Xi_{1}, \Xi_{2}, \cdots, \Xi_{n}\right\}$. Uma estrutura homoclínica em $\mathcal{A}$ relativa $a \div$ consiste em um subconjunto $\left\{\Xi_{l_{1}}, \Xi_{l_{2}}, \cdots, \Xi_{l_{k}}\right\} \subset \Xi$ em conjunto com uma família de soluções globais $\left\{\xi_{j}: \mathbb{R} \rightarrow X: j=1, \cdots, k\right\}$ tais que, pondo $\Xi_{l_{(k+1)}}:=\Xi_{l_{1}}$, para todo $j=1,2, \cdots, k$, tem-se:

(i) Para cada $j$ existe $t_{j} \in \mathbb{R}$ com $\xi_{j}\left(t_{j}\right) \notin\left(\Xi_{l_{j}} \cup \Xi_{l_{(j+1)}}\right)$ e

(ii)

$$
\lim _{t \rightarrow-\infty} d\left(\xi_{j}(t), \Xi_{l_{j}}\right)=0 \text { e } \lim _{t \rightarrow \infty} d\left(\xi_{j}(t), \Xi_{l_{(j+1)}}\right)=0 .
$$


Uma estrutura homoclínica pode ser entendida como uma espécie de polígono contido em $\mathcal{A}$, onde os vértices estão representados pelos conjuntos invariantes isolados e as arestas representadas pelas órbitas das soluções globais que os conectam, mas sempre levando em conta uma certa "orientação" nas arestas quando se passa de um vértice a outro.

Observemos que a condição: "Para cada $j$ existe $t_{j} \in \mathbb{R} \operatorname{com} \xi_{j}\left(t_{j}\right) \notin\left(\Xi_{l_{j}} \cup \Xi_{l_{(j+1)}}\right)$ ", exigida no item $(i)$ da definição acima, somente é necessária no caso em que se tem $k=1$, uma vez que, sendo $\boldsymbol{\Xi}$ uma família finita disjunta de invariantes isolados, é evidente que quando (2.2.4) se verifica, a solução $\xi_{j}$, devido a sua continuidade, deve deixar ambos os conjuntos $\Xi_{l_{j}}$ e $\Xi_{l_{(j+1)}}$ em algum instante $t_{j}$.

Proposição 2.2.2. Sejam $\{T(t): t \geq 0\}$ um semigrupo gradiente generalizado com respeito à uma familia finita disjunta de conjuntos invariantes isolados limitados $\boldsymbol{\Xi}:=$ $\left\{\Xi_{1}, \Xi_{2}, \cdots, \Xi_{n}\right\}$, possuindo atrator global $\mathcal{A}$ e $V: X \rightarrow \mathbb{R}$ sua função de Lyapunov correspondente.

Verificam-se as duas propriedades seguintes:

(i) Se $\xi: \mathbb{R} \rightarrow X$ é uma solução global limitada de $T(\cdot)$, existem indices $i, j \in$ $\{1,2, \cdots, n\}$ tais que

$$
\lim _{t \rightarrow-\infty} d\left(\xi(t), \Xi_{i}\right)=0 \text { e } \lim _{t \rightarrow \infty} d\left(\xi(t), \Xi_{j}\right)=0 .
$$

(ii) O atrator $\mathcal{A}$ não possui estruturas homoclínicas relativas a $\boldsymbol{\Xi}$.

Demonstração: Para provar $(i)$ consideremos $\xi: \mathbb{R} \rightarrow X$ uma solução global limitada para $\{T(t): t \geq 0\}$ então, graças a $(i i)$ na Definição 2.1.3, a função $\mathbb{R} \ni t \mapsto$ $V(\xi(t)) \in \mathbb{R}$ é monótona e pelo fato de que $\xi(t)$ está no compacto $\mathcal{A}$ para todo $t \in \mathbb{R}$, segue-se a existência dos limites

$$
L:=\lim _{t \rightarrow-\infty} V(\xi(t)) \text { e } l:=\lim _{t \rightarrow \infty} V(\xi(t))
$$

Sejam $\alpha(\xi)$ e $\omega(\xi)$, respectivamente, os conjuntos $\alpha$ e $\omega$-limites associados à solução $\xi$. De (2.2.5) acima e da caracterização dos conjuntos limites em termos de limites de sequências, segue-se que $V$, restrita a $\alpha(\xi)$, é constante igual a $L$ e, por sua vez, restrita a $\omega(\xi)$ é constante igual a $l$. Então, dado $x \in \omega(\xi)$, como $\omega(\xi)$ é invariante, $T(t) x \in \omega(\xi)$ para todo $t \geq 0$, donde $V(x)=V(T(t) x)=l$ para todo $t \geq 0$ e por $($ iii $)$ da Definição 2.1.3, isto obriga $x$ a estar em $\Xi_{j}$ para algum $j \in\{1,2, \cdots, n\}$.

Agora, da conexidade do conjunto $\omega(\xi)$ e do fato de que os conjuntos em $\Xi$ são isolados entre si, é fácil ver que $\omega(\xi)$ está inteiramente contido em $\Xi_{j}$ e, analogamente, conclui-se que $\alpha(\xi)$ está contido em $\Xi_{i}$ para algum $i \in\{1,2, \cdots, n\}$. Assim, levando em 
conta as propriedades de atração que possuem os conjuntos $\alpha$ e $\omega$-limites, terminamos a prova do item $(i)$ desta proposição.

Para o item $(i i)$, sejam $\left\{\Xi_{l_{1}}, \cdots, \Xi_{l_{k}}\right\} \subset \Xi$ e $\left\{\xi_{j}: \mathbb{R} \rightarrow X: j=1, \cdots, k\right\}$, um conjunto de soluções globais, formando uma estrutura homoclínica relativa a $\boldsymbol{\Xi}$, isto é, pondo $\Xi_{l_{(k+1)}}:=\Xi_{l_{1}}$, tem-se para cada $j=1,2, \cdots, k$

$$
\lim _{t \rightarrow-\infty} d\left(\xi_{j}(t), \Xi_{l_{j}}\right)=0 \text { e } \lim _{t \rightarrow \infty} d\left(\xi_{j}(t), \Xi_{l_{(j+1)}}\right)=0 .
$$

Sejam, de acordo com a propriedade ( $i v$ ) da Definição 2.1 .3 , para cada $j=1,2, \cdots, k+$ $1, L_{j}$ o valor constante que a função $V$ assume sobre o conjunto $\Xi_{l_{j}}$. Da continuidade de $V$ e da propriedade ( $i i$ ) da Definição 2.1 .3 resulta $L_{1} \geq L_{2} \geq \cdots \geq L_{k} \geq L_{k+1}=L_{1}$, logo todos os $L_{j}$ 's devem ser iguais entre si e, digamos, que valham $L$.

Por outro lado, fixado $j=1,2, \cdots, k$ como a solução $\xi_{j}$ é tal que $L_{j} \geq V\left(\xi_{j}(t)\right) \geq$ $L_{j+1}$, para todo $t \in \mathbb{R}$, e $L_{j}=L_{j+1}=L$, então, para todo real $s V\left(T(t) \xi_{j}(s)\right)=$ $V\left(\xi_{j}(t+s)\right)=L=V\left(\xi_{j}(s)\right)$ para todo $t \geq 0$. Agora, a condição (iii) da Definição 2.1.3 diz que, nestas condições, $\xi_{j}(s) \in \Xi$ para algum $\Xi \in \Xi$, donde conclui-se facilmente que, na verdade, $\xi(t) \in \Xi$ para todo $t \in \mathbb{R}$. Logo, por $(2.2 .6), \Xi_{l_{j}}=\Xi_{l_{(j+1)}}=\Xi$, pela arbitrariedade com a qual escolhemos $j=1,2, \cdots, k$, conclui-se que todos os invariantes $\Xi_{l_{1}}, \cdots, \Xi_{l_{k}}$ são iguais entre si, digamos iguais a $\Xi$, e que, portanto, todas as soluções $\xi_{j}: \mathbb{R} \rightarrow X, j=1, \cdots, k$ estão contidas em $\Xi$, o que está em contradição com o fato de que $\left\{\Xi_{l_{1}}, \cdots, \Xi_{l_{k}}\right\} \subset \boldsymbol{\Xi}$ juntamente com $\left\{\xi_{j}: \mathbb{R} \rightarrow X: j=1, \cdots, k\right\}$ constituem uma estrutura homoclínica, estabelecendo a propriedade (ii) e completando a demonstração.

Com base na proposição anterior e no Corolário 1.1.7 podemos obter uma informação adicional sobre a estrutura geométrica dos atratores dos semigrupos gradientes. Para isso, devemos definir os conceitos de conjuntos estáveis e instáveis associados a um conjunto invariante.

Definição 2.2.3. Sejam $\{T(t): t \geq 0\}$ um semigrupo em um espaço métrico $X$ e $\Xi$ um conjunto invariante. Define-se:

(a) O conjunto instável de $\Xi$ como

$$
\begin{gathered}
W^{u}(\Xi):=\{x \in X \text { : existe } \xi: \mathbb{R} \rightarrow X \text {, solução global, com } \xi(0)=x \\
\text { tal que } \left.\lim _{t \rightarrow-\infty} d(\xi(t), \Xi)=0\right\} .
\end{gathered}
$$

(b) O conjunto estável de $\Xi$ como

$$
W^{s}(\Xi):=\left\{x \in X: \lim _{t \rightarrow \infty} d(T(t) x, \Xi)=0\right\} .
$$


Observemos que, se $x \in W^{u}(\Xi)$ e $\xi: \mathbb{R} \rightarrow X$ é uma solução global para $T(\cdot)$ com $\xi(0)=x$ e $\lim _{t \rightarrow-\infty} d(\xi(t), \Xi)=0$, então $\xi(s) \in W^{u}(\Xi)$ para todo real $s$, uma vez que, dado $s \in \mathbb{R}$, definindo $\xi_{s}: \mathbb{R} \rightarrow X$ por $\xi_{s}(t):=\xi(t+s)$ para cada real $t$, é imediato que $\xi_{s}: \mathbb{R} \rightarrow X$ é solução global para $T(\cdot)$ e, além disso, satisfaz $\lim _{t \rightarrow-\infty} d\left(\xi_{s}(t), \Xi\right)=$ $\lim _{t \rightarrow-\infty} d(\xi(t+s), \Xi)=0, \operatorname{com} \xi_{s}(0)=\xi(s)$.

Corolário 2.2.4. Sejam $\{T(t): t \geq 0\}$ um semigrupo gradiente generalizado com respeito à família finita disjunta de conjuntos invariantes isolados limitados $\boldsymbol{\Xi}:=$ $\left\{\Xi_{1}, \Xi_{2}, \cdots, \Xi_{n}\right\}$. Se $\{T(t): t \geq 0\}$ possui atrator global $\mathcal{A}$, então $\mathcal{A}$ é a reunião dos conjuntos instáveis dos conjuntos invariantes isolados pertencentes a $\boldsymbol{\Xi}$. Simbolicamente:

$$
\mathcal{A}=\bigcup_{j=1}^{n} W^{u}\left(\Xi_{j}\right)
$$

Demonstração: Com efeito, por um lado, do Corolário 1.1.7, temos que $\mathcal{A}$ é a reunião de todas as órbitas globais limitadas de $T(\cdot)$, então, se $\xi: \mathbb{R} \rightarrow X$ é uma solução global limitada, $(i)$ da Proposição 2.2.2 assegura que a ela está associado um elemento $\Xi \in \Xi$ de maneira que $\lim _{t \rightarrow-\infty} d(\xi(t), \Xi)=0$, o que significa, como observamos acima, que para todo real $t, \xi(t) \in W^{u}(\Xi)$, estabelecendo a inclusão $\mathcal{A} \subset \bigcup_{j=1}^{n} W^{u}\left(\Xi_{j}\right)$.

Reciprocamente, sejam $x \in W^{u}(\Xi)$, para algum $\Xi \in \Xi$, e $\xi: \mathbb{R} \rightarrow X$ uma solução global para $T(\cdot) \operatorname{com} \xi(0)=x$ e $\lim _{t \rightarrow-\infty} d(\xi(t), \Xi)=0$, como $\Xi$ é limitado, segue-se que para todo real $\tau$ o conjunto $\{\xi(t): t \leq \tau\}$ é limitado e como já vimos, para todo real $\tau$ o conjunto $\{\xi(t): t \geq \tau\}$ é também limitado, mostrando que a solução $\xi$ tem órbita limitada, donde segue, pelo Corolário 1.1.7, que tal órbita está contida em $\mathcal{A}$ e, em particular, $x \in \mathcal{A}$, dizendo que a inclusão $\bigcup_{j=1}^{n} W^{u}\left(\Xi_{j}\right) \subset \mathcal{A}$ também se verifica, terminando a demonstração.

Se um semigrupo $T(\cdot)$ possui atrator global $\mathcal{A}$ e uma família finita disjunta de conjuntos invariantes isolados $\Xi=\left\{\Xi_{1}, \cdots, \Xi_{n}\right\}$ de maneira que $\mathcal{A}$ admite a representação dada em (2.2.7), costuma-se dizer que $T(\cdot)$ possui atrator de tipo gradiente.

\subsection{Existência de atratores para semigrupos gradientes}

Terminamos este capítulo provando um resultado sobre existência de atrator no caso especial em que o semigrupo em estudo é um semigrupo gradiente. A idéia de nossa demonstração está, de certa forma, inspirada na que se encontra em [16]. 
Teorema 2.3.1. Seja $\{T(t): t \geq 0\}$ um semigrupo gradiente generalizado com respeito a uma familia finita disjunta de conjuntos invariantes isolados limitados $\boldsymbol{\Xi}:=$ $\left\{\Xi_{1}, \Xi_{2}, \cdots, \Xi_{n}\right\}$. Se $\{T(t): t \geq 0\}$ é um semigrupo eventualmente compacto e eventualmente limitado, então ele possui atrator global.

Demonstração: Graças ao Corolário 1.3.2, é suficiente provarmos que $T(\cdot)$ é ponto dissipativo.

Com efeito, consideremos o conjunto limitado

$$
D_{0}:=\bigcup_{j=1}^{n} \mathcal{O}_{1}\left(\Xi_{j}\right) .
$$

Então, por um argumento inteiramente análogo ao da demonstração da Proposição 2.2.2 (mais precisamente quando se prova que para toda solução global limitada $\xi$ : $\mathbb{R} \rightarrow X$, tem-se $\omega(\xi) \subset \Xi_{j}$ para algum $\left.\Xi_{j} \in \Xi\right)$, se vê que $D_{0}$ é um limitado que absorve pontos de $X$ pela ação de $T(\cdot)$ é o teorema fica provado. 


\section{Capítulo}

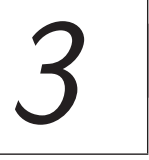

\section{Semigrupos de tipo Gradiente}

Agora apresentamos alguns elementos da teoria dos semigrupos de tipo gradiente, que foi desenvolvida e introduzida em [8]. Esta classe de semigrupos é definida com base na dinâmica dos semigrupos gradientes que estudamos no capítulo anterior.

Um semigrupo de tipo gradiente é um semigrupo que satisfaz as conclusões da Proposição 2.2.2, ou seja, um cuja dinâmica imita a de um semigrupo gradiente. A importância em se estudar esta classe de semigrupos reside no fato de que são estáveis por perturbação. Em outras palavras, a dinâmica de um sistema gradiente é estável por perturbação, dizendo que a existência da função de Lyapunov pode ser suprimida se o objeto principal de estudo é, por exemplo, a estrutura do atrator. Contudo, de forma surpreendente, estabelecemos, no próximo capítulo, que a dinâmica dos semigrupos gradientes é autosuficiente no sentido de que a própria existência da função de Lyapunov se converte em uma consequência de suas propriedades dinâmicas.

\subsection{Definição}

De forma precisa podemos definir o conceito como segue.

Definição 3.1.1. Seja $\{T(t): t \geq 0\}$ um semigrupo em um espaço métrico $X$ possuindo atrator global $\mathcal{A}$ e uma família finita disjunta de conjuntos invariantes isolados limitados $\Xi:=\left\{\Xi_{1}, \Xi_{2}, \cdots, \Xi_{n}\right\}$. Diz-se que $\{T(t): t \geq 0\}$ é um semigrupo de tipo gradiente generalizado com respeito à família $\Xi$ quando verificam-se as seguintes propriedades: 
(G1) Para toda solução global limitada $\xi: \mathbb{R} \rightarrow X$ existem índices $i, j \in\{1,2, \cdots, n\}$ tais que

$$
\lim _{t \rightarrow-\infty} d\left(\xi(t), \Xi_{i}\right)=0 e \lim _{t \rightarrow \infty} d\left(\xi(t), \Xi_{j}\right)=0 .
$$

(G2) O atrator $\mathcal{A}$ não contém estruturas homoclínicas relativas a $\boldsymbol{\Xi}$.

Por abuso de notação, as vezes, diremos que o par $(T(\cdot), \boldsymbol{\Xi})$ é um semigrupo de tipo gradiente.

\subsection{Estabilidade por perturbação}

Nesta seção estabelecemos a estabilidade por perturbação dos semigrupos de tipo gradiente. Para isso, é necessário trabalhar com famílias de semigrupos. Nesta direção, comecemos introduzindo o que se conhece como compacidade assintótica coletiva.

Definição 3.2.1. Uma família de semigrupos em um espaço métrico $X,\left\{T_{\eta}(t): t \geq 0\right\}$ para $\eta \in[0,1]$, é coletivamente assintoticamente compacta quando, para cada sequência $\left(\eta_{k}\right)_{k \in \mathbb{N}}$ em $[0,1]$ com $\eta_{k} \rightarrow 0$, cada sequência limitada $\left(x_{k}\right)_{k \in \mathbb{N}}$ em X e cada sequência $\left(t_{k}\right)_{k \in \mathbb{N}}$ de números reais positivos com $t_{k} \rightarrow \infty$, a sequência $\left(T_{\eta_{k}}\left(t_{k}\right) x_{k}\right)_{k \in \mathbb{N}}$ possui uma subsequência convergente.

Uma das hipóteses do teorema de perturbação de semigrupos de tipo gradiente é a convergência uniforme sobre compactos da família de semigrupos em estudo. Deixemos aqui registrada a seguinte definição.

Definição 3.2.2. Seja $\left\{T_{\eta}(t): t \geq 0\right\}_{\eta \in(0,1]}$ uma família de semigrupos em um espaço métrico $X:=(X, d)$. Diz-se que $\left(T_{\eta}\right)_{\eta \in(0,1]}$ converge uniformemente sobre compactos a um semigrupo $\left\{T_{0}(t): t \geq 0\right\}$ quando $\eta$ tende a zero, e indica-se este fato por $T_{\eta} \underset{\eta \rightarrow 0^{+}}{\rightarrow} T_{0}$, quando para todo $\tau>0$ e todo subconjunto compacto $K \subset X$ tem-se

$$
\lim _{\eta \rightarrow 0^{+}} \sup _{x \in K} \sup _{t \in[0, \tau]} d\left(T_{\eta}(t) x, T_{0}(t) x\right)=0
$$

Supondo estas duas propriedades verificadas para uma certa família $\left\{T_{\eta}(t): t \geq\right.$ $0\}_{\eta \in[0,1]}$ de semigrupos, podemos provar o seguinte resultado, o qual será de fundamental importância para tudo o que segue neste texto.

Lema 3.2.3. Seja $\left\{T_{\eta}(t): t \geq 0\right\}_{\eta \in[0,1]}$ uma família coletivamente assintoticamente compacta de semigrupos em um espaço métrico $X$, convergindo uniformemente sobre compactos ao semigrupo $\left\{T_{0}(t): t \geq 0\right\}$. 
Sejam também, $\left(\eta_{k}\right)_{k \in \mathbb{N}}$ uma sequência em $[0,1] \operatorname{com} \eta_{k} \rightarrow 0$ e $\left(I_{k}\right)_{k \in \mathbb{N}}$ uma sequência de intervalos da reta real de modo que, para cada $k \in \mathbb{N}, I_{k}:=\left[-t_{k}, \infty\right)$ para uma certa sequência crescente de números positivos $\left(t_{k}\right)_{k \in \mathbb{N}}$ com $t_{k} \rightarrow \infty$.

Nestas condições, se $\left(x_{k}\right)_{k \in \mathbb{N}}$ é uma sequência limitada de pontos de $X$, definindo para cada natural $k, \xi_{k}: I_{k} \rightarrow X$ por

$$
\xi_{k}(t):=T_{\eta_{k}}\left(t+t_{k}\right) x_{k}, t \in I_{k},
$$

existem $\xi_{0}: \mathbb{R} \rightarrow X$ uma solução global para $\left\{T_{0}(t): t \geq 0\right\}$ e uma subsequência de $\left(\xi_{k}\right)_{k \in \mathbb{N}}$ convergindo uniformemente sobre compactos da reta para a solução $\xi_{0}$.

Demonstração: Com efeito, pela compacidade assintótica coletiva da família $\left\{T_{\eta}(t)\right.$ : $t \geq 0\}_{\eta \in[0,1]}$, seja $\mathbb{N}_{0} \subset \mathbb{N}$ um subconjunto infinito de números naturais tal que a sequência $\left(T_{\eta_{k}}\left(t_{k}\right) x_{k}\right)_{k \in \mathbb{N}_{0}}$ converge para um ponto $z_{0} \in X$. Definamos

$$
\xi_{0}^{(0)}(t):=T_{0}(t) z_{0} \text { para } t \geq 0
$$

Agora, consideremos, pela mesma razão de antes, $\mathbb{N}_{1} \subset \mathbb{N}_{0}$ um subconjunto infinito tal que $t_{k}>1$ para todo $k \in \mathbb{N}_{1}$ e a sequência $\left(T_{\eta_{k}}\left(t_{k}-1\right) x_{k}\right)_{k \in \mathbb{N}_{1}}$ converge para um ponto $z_{1} \in X$. Definamos

$$
\xi_{0}^{(1)}(t):=T_{0}(t+1) z_{1} \text { para } t \in[-1,0] .
$$

Observemos, pelo fato de que se uma sequência converge então todas as suas subsequências convergem ao mesmo limite, que

$$
\begin{aligned}
\xi_{0}^{(1)}(0)= & T_{0}(1) z_{1}=\lim _{\substack{k \rightarrow \infty \\
k \in \mathbb{N}_{1}}} T_{\eta_{k}}(1) T_{\eta_{k}}\left(t_{k}-1\right) x_{k}= \\
& \lim _{\substack{k \rightarrow \infty \\
k \in \mathbb{N}_{0}}} T_{\eta_{k}}\left(t_{k}\right) x_{k}=z_{0}=\xi_{0}^{(0)}(0) .
\end{aligned}
$$

Analogamente, seja $\mathbb{N}_{2} \subset \mathbb{N}_{1}$ um subconjunto infinito tal que $t_{k}>2$ para todo $k \in \mathbb{N}_{2}$ e a sequência $\left(T_{\eta_{k}}\left(t_{k}-2\right) x_{k}\right)_{k \in \mathbb{N}_{2}}$ converge a um ponto $z_{2} \in X$. Definamos

$$
\xi_{0}^{(2)}(t):=T_{0}(t+2) z_{2} \text { para } t \in[-2,-1]
$$

e, como antes, notemos que

$$
\begin{gathered}
\xi_{0}^{(2)}(-1)=T_{0}(1) z_{2}=\lim _{\substack{k \rightarrow \infty \\
k \in \mathbb{N}_{2}}} T_{\eta_{k}}(1) T_{\eta_{k}}\left(t_{k}-2\right) x_{k}= \\
\lim _{\substack{k \rightarrow \infty \\
k \in \mathbb{N}_{1}}} T_{\eta_{k}}\left(t_{k}-1\right) x_{k}=z_{1}=\xi_{0}^{(1)}(-1) .
\end{gathered}
$$


Repetindo o argumento, obtemos uma cadeia decrescente de conjuntos infinitos de números naturais

$$
\mathbb{N} \supset \mathbb{N}_{0} \supset \mathbb{N}_{1} \supset \cdots \supset \mathbb{N}_{n} \supset \cdots
$$

de maneira que para cada $n=0,1,2, \cdots$ existe um ponto $z_{n} \in X$ tal que

$$
z_{n}=\lim _{\substack{k \rightarrow \infty \\ k \in \mathbb{N}_{n}}} T_{\eta_{k}}\left(t_{k}-n\right) x_{k}
$$

e definindo a aplicação

$$
\xi_{0}^{(n)}(t):=T_{0}(t+n) z_{n} \text { para } t \in[-n, 1-n]
$$

vemos que

$$
\xi_{0}^{(n)}(1-n)=\xi_{0}^{(n-1)}(1-n) \text { sempre que } n \in \mathbb{N} .
$$

Baseados nestes fatos definimos $\xi_{0}: \mathbb{R} \rightarrow X$ pondo

$$
\xi_{0}(t):=\left\{\begin{array}{l}
\xi_{0}^{(0)}(t), t \geq 0 \\
\xi_{0}^{(n)}(t), t \in[-n, 1-n], n \in \mathbb{N}
\end{array}\right.
$$

e observemos, de (3.2.1), que $\xi_{0}: \mathbb{R} \rightarrow X$ está definida sem ambiguidades.

Agora, afirmamos que $\xi_{0}: \mathbb{R} \rightarrow X$ é solução global de $T_{0}(\cdot)$.

De fato, em primeiro lugar, dados $t, \tau \geq 0$, simplesmente aplicando a definição de $\xi_{0}$, temos que

$$
T_{0}(t) \xi_{0}(\tau)=T_{0}(t) T_{0}(\tau) z_{0}=T_{0}(t+\tau) z_{0}=\xi_{0}(\tau+t)
$$

porque $\tau+t \geq 0$.

Agora, se $\tau<0$ podemos escolher $n \in \mathbb{N}$ de modo que $\tau \in[-n, 1-n]$, donde $\xi_{0}(\tau)=T_{0}(\tau+n) z_{n}$. Caso seja $t+\tau \geq 0$, então

$$
\begin{gathered}
T_{0}(t) \xi_{0}(\tau)=T_{0}(t) T_{0}(\tau+n) z_{n}=T_{0}([t+\tau]+n) z_{n}= \\
T_{0}(t+\tau) T_{0}(n) z_{n}=T_{0}(t+\tau) z_{0}=\xi_{0}(\tau+t),
\end{gathered}
$$

pois, como é fácil comprovar, $T_{0}(n) z_{n}=z_{0}$.

Caso $\tau+t<0$, existe um natural $m \leq n$ de maneira que $\tau+t \in[-m, 1-m]$ e assim obtém-se, levando em conta que $T_{0}(n-m) z_{n}=z_{m}$,

$$
\begin{gathered}
T_{0}(t) \xi_{0}(\tau)=T_{0}(t) T_{0}(\tau+n) z_{n}=T_{0}([t+\tau+m]+[n-m]) z_{n}= \\
T_{0}(t+\tau+m) T_{0}(n-m) z_{n}=T_{0}(t+\tau+m) z_{m}=\xi_{0}^{(m)}(\tau+t)=\xi_{0}(\tau+t),
\end{gathered}
$$

donde resulta que $\xi_{0}: \mathbb{R} \rightarrow X$ é solução global de $T_{0}(\cdot)$. 
Por outro lado, definindo o conjunto $\mathbb{N}^{*}$ de modo que seu $n$-ésimo elemento seja o $n$-ésimo elemento do conjunto $\mathbb{N}_{n}$, na ordem crescente dos números naturais, vemos que $\mathbb{N}^{*}$ é um conjunto infinito e, portanto, considerando a restrição $\left(\xi_{n}\right)_{n \in \mathbb{N}^{*}}$, segue-se que $\left(\xi_{n}\right)_{n \in \mathbb{N}^{*}}$ é uma subsequência de $\left(\xi_{n}\right)_{n \in \mathbb{N}}$ que converge uniformemente sobre compactos da reta para a solução $\xi_{0}: \mathbb{R} \rightarrow X$.

Com efeito, em primeiro lugar, sejam $0 \leq a<b$ dois reais dados. Então, a hipótese de convergência dos semigrupos nos diz que

$$
\lim _{k \rightarrow \infty} \sup _{x \in C_{0}} \sup _{t \in[a, b]} d\left(T_{\eta_{k}}(t) x, T_{0}(t) x\right)=0,
$$

onde $C_{0}:=\left\{T_{\eta_{k}}\left(t_{k}\right) x_{k}: k \in \mathbb{N}_{0}\right\}$ e por isto, como para todo natural $k$ tem-se

$$
\begin{gathered}
d\left(T_{\eta_{k}}\left(t+t_{k}\right) x_{k}, T_{0}(t) z_{0}\right) \leq d\left(T_{\eta_{k}}(t) T_{\eta_{k}}\left(t_{k}\right) x_{k}, T_{0}(t) T_{\eta_{k}}\left(t_{k}\right) x_{k}\right)+ \\
d\left(T_{0}(t) T_{\eta_{k}}\left(t_{k}\right) x_{k}, T_{0}(t) z_{0}\right),
\end{gathered}
$$

segue-se que

$$
\lim _{\substack{k \rightarrow \infty \\ k \in \mathbb{N}_{0}}} \sup _{t \in[a, b]} d\left(T_{\eta_{k}}\left(t+t_{k}\right) x_{k}, T_{0}(t) z_{0}\right)=0
$$

$\log 0$

$$
\lim _{\substack{k \rightarrow \infty \\ k \in \mathbb{N}_{0}}} \sup _{t \in[a, b]} d\left(\xi_{k}(t), \xi_{0}(t)\right)=0 .
$$

Agora, fixado um natural $n$, obtém-se

$$
\lim _{k \rightarrow \infty} \sup _{x \in C_{n}} \sup _{t \in[-n, 1-n]} d\left(T_{\eta_{k}}(t) x, T_{0}(t) x\right)=0
$$

onde $C_{n}:=\left\{T_{\eta_{k}}\left(t_{k}-n\right) x_{k}: k \in \mathbb{N}_{n}\right\}$ e, analogamente ao que fizemos antes, como para todo $k \in \mathbb{N}_{n}$ e $t \in[-n, 1-n]$

$$
\begin{gathered}
d\left(T_{\eta_{k}}\left(t+t_{k}\right) x_{k}, T_{0}(t+n) z_{n}\right) \leq \\
d\left(T_{\eta_{k}}(t+n) T_{\eta_{k}}\left(t_{k}-n\right) x_{k}, T_{0}(t+n) T_{\eta_{k}}\left(t_{k}-n\right) x_{k}\right)+ \\
d\left(T_{0}(t+n) T_{\eta_{k}}\left(t_{k}-n\right) x_{k}, T_{0}(t+n) z_{n}\right)
\end{gathered}
$$

vemos que

$$
\lim _{\substack{k \rightarrow \infty \\ k \in \mathbb{N}_{n}}} \sup _{t \in[-n, 1-n]} d\left(T_{\eta_{k}}\left(t+t_{k}\right) x_{k}, T_{0}(t+n) z_{n}\right)=0
$$

e assim

$$
\lim _{\substack{k \rightarrow \infty \\ k \in \mathbb{N}_{n}}} \sup _{t \in[-n, 1-n]} d\left(\xi_{k}(t), \xi_{0}(t)\right)=0 .
$$

Finalmente, o caso geral segue dos considerados anteriormente, pois todo compacto $K \subset \mathbb{R}$ está contido em uma reunião finita de intervalos da forma que consideramos acima e observando que, para cada natural $n$ a sequência $\left(\xi_{k}\right)_{k \in \mathbb{N}^{*}}$ é, a partir do seu $n$-ésimo termo, uma subsequência de $\left(\xi_{k}\right)_{k \in \mathbb{N}_{n}}$ o lema está provado. 
Agora estamos em condições de enunciar e provar o principal resultado deste capítulo, que estabelece condições suficientes para a estabilidade por perturbação dos semigrupos de tipo gradiente e que foi provado em [8] (Confira o Theorem 1.5).

Teorema 3.2.4 (Carvalho e Langa). Seja $\left\{T_{\eta}(t): t \geq 0\right\}_{\eta \in[0,1]}$ uma família coletivamente assintoticamente compacta de semigrupos num espaço métrico $X$ convergindo uniformemente sobre compactos ao semigrupo $\left\{T_{0}(t): t \geq 0\right\}$.

Suponhamos que se verificam as seguintes quatro hipóteses:

(a) Para cada $\eta \in[0,1]$, o semigrupo $\left\{T_{\eta}(t): t \geq 0\right\}$ possui atrator global $\mathcal{A}_{\eta}$ e, além disso, a reunião $\bigcup_{\eta \in[0,1]} \mathcal{A}_{\eta}$ é limitada.

(b) Existe um natural $n$ tal que para cada $\eta \in[0,1]$ o semigrupo $\left\{T_{\eta}(t): t \geq 0\right\}$ possui uma família finita disjunta de conjuntos invariantes isolados limitados,

$$
\Xi_{\eta}:=\left\{\Xi_{1, \eta}, \Xi_{2, \eta}, \cdots, \Xi_{n, \eta}\right\}
$$

que se comportam continuamente em $\eta=0$, isto é, para cada $i=1,2, \cdots, n$ tem-se

$$
\lim _{\eta \rightarrow 0^{+}} d_{H}\left(\Xi_{i, \eta}, \Xi_{i, 0}\right)=0
$$

(c) Existe $\delta_{0}>0$ tal que para todo $i=1,2, \cdots, n$ e todo $\eta \in[0,1]$, tem-se que $\Xi_{i, \eta}$ é o invariante maximal para $T_{\eta}(\cdot)$ em $\mathcal{O}_{\delta_{0}}\left(\Xi_{i, 0}\right)$.

(d) O semigrupo $\left\{T_{0}(t): t \geq 0\right\}$ é de tipo gradiente generalizado com respeito à familia $\Xi_{0}=\left\{\Xi_{1,0}, \Xi_{2,0}, \cdots, \Xi_{n, 0}\right\}$.

Nestas condições, existe $\eta_{0} \in(0,1]$ tal que para todo $\eta \in\left[0, \eta_{0}\right]$ o semigrupo $\left\{T_{\eta}(t): t \geq 0\right\}$ é de tipo gradiente generalizado com respeito à família $\boldsymbol{\Xi}_{\eta}=\left\{\Xi_{1, \eta}, \cdots, \Xi_{n, \eta}\right\}$.

Demonstração: Comecemos provando que a propriedade $(G 1)$ é estável por perturbação, ou seja, que existe $\eta_{1} \in(0,1]$ de maneira que para todo $\eta \in\left[0, \eta_{1}\right]$ o semigrupo $\left(T_{\eta}(\cdot), \boldsymbol{\Xi}_{\eta}\right)$ satisfaz $(G 1)$.

Com efeito, em primeiro lugar, observemos que, da hipótese (b) obtemos facilmente a existência de $\delta \in\left(0, \delta_{0}\right)$, onde $\delta_{0}>0$ é tal que as $\delta_{0}$-vizinhanças dos conjuntos em $\Xi_{0}$ são disjuntas entre si, e um $\eta^{\prime} \in(0,1]$ tal que, se para $\eta \in\left[0, \eta^{\prime}\right], \xi_{\eta}: \mathbb{R} \rightarrow X$ é solução global para $T_{\eta}(\cdot)$ e existe $t_{\eta} \in \mathbb{R}$ de modo que

$$
d\left(\xi_{\eta}(t), \bigcup_{i=1}^{n} \Xi_{i, 0}\right)<\delta \text { para todo } t \geq t_{\eta},
$$


então, simplesmente da maximalidade dos invariantes e da condição de continuidade das famílias $\boldsymbol{\Xi}_{\eta}$, conclui-se que

$$
\lim _{t \rightarrow \infty} d\left(\xi_{\eta}(t), \bigcup_{i=1}^{n} \Xi_{i, \eta}\right)=0 .
$$

Levando em conta esta observação, afirmamos que existe $\eta_{1} \in\left(0, \eta^{\prime}\right]$ tal que para $\eta \in\left[0, \eta_{1}\right]$ se $\xi_{\eta}: \mathbb{R} \rightarrow X$ é solução global limitada para $T_{\eta}(\cdot)$, então existe $t_{\eta} \in \mathbb{R}$ de modo que

$$
\sup _{t \geq t_{\eta}} d\left(\xi_{\eta}(t), \bigcup_{i=1}^{n} \Xi_{i, 0}\right)<\delta .
$$

Observemos que, pelo que dissemos acima, se isto for certo, existirá $\eta_{1}>0$ de modo que toda solução global limitada de $T_{\eta}(\cdot)$, com $\eta \in\left[0, \eta_{1}\right]$, convergirá, quando $t \rightarrow \infty$, a um elemento de $\boldsymbol{\Xi}_{\eta}$.

Com efeito, se a afirmação acima não se verifica, obtém-se a existência de uma sequência $\left(\eta_{k}\right)_{k \in \mathbb{N}}$ em $(0,1]$, com $\eta_{k} \rightarrow 0^{+}$, e para cada natural $k$ uma solução $\xi_{k}: \mathbb{R} \rightarrow$ $X$ de $T_{\eta_{k}}(\cdot)$, de maneira que, para todo natural $k$ e todo real $s$, tem-se

$$
\sup _{t \geq s} d\left(\xi_{k}(t), \bigcup_{i=1}^{n} \Xi_{i, 0}\right) \geq \delta .
$$

Por outro lado, usando um argumento semelhante ao que usamos para provar o Lema 3.2.3, podemos supor a existência de uma solução global $\xi^{(0)}: \mathbb{R} \rightarrow X$ de $T_{0}(\cdot)$ tal $\xi_{k} \underset{k \rightarrow \infty}{\rightarrow} \xi^{(0)}$ uniformemente sobre compactos da reta, e do fato de que $T_{0}(\cdot)$ é de tipo gradiente, segue-se a existência de um invariante isolado $\Xi_{i_{0}, 0} \in \boldsymbol{\Xi}_{0}$ tal que

$$
\lim _{t \rightarrow \infty} d\left(\xi^{(0)}(t), \Xi_{i_{0}, 0}\right)=0
$$

Então, dado $r \in \mathbb{N}\left(\operatorname{com} \frac{1}{r}<\delta\right)$ existem $k_{r} \in \mathbb{N}$ e $t_{r} \in \mathbb{R}$ de modo que

$$
d\left(\xi_{k}\left(t_{r}\right), \Xi_{i_{0}, 0}\right)<\frac{1}{r} \text { sempre que } k \geq k_{r}
$$

e, de (3.2.3), resulta a existência de $t_{r}^{\prime}>t_{r}$ tal que, pelo Teorema do Valor Intermediário, temos

$$
d\left(\xi_{k_{r}}(t), \Xi_{i_{0}, 0}\right)<\delta \text { para todo } t \in\left[t_{r}, t_{r}^{\prime}\right)
$$

$\mathrm{e}$

$$
d\left(\xi_{k_{r}}\left(t_{r}^{\prime}\right), \Xi_{i_{0}, 0}\right)=\delta .
$$

Nestas condições, afirmamos que $t_{r}^{\prime}-t_{r} \rightarrow \infty$.

Com efeito, caso contrário, podemos supor que $t_{r}^{\prime}-t_{r} \underset{r \rightarrow \infty}{\rightarrow} t_{0}$, para algum $t_{0} \geq 0$, e então, para todo $r, \xi_{k_{r}}\left(t_{r}^{\prime}\right)=T_{\eta_{k}}\left(t_{r}^{\prime}-t_{r}\right) \xi_{k_{r}}\left(t_{r}\right)$ donde resultaria, após fazer $r \rightarrow \infty$ e 
levando em conta (3.2.4), a existência de um ponto em $\Xi_{i_{0}, 0}$ distando $\delta>0$ de $\Xi_{i_{0}, 0}$, o que obviamente é um absurdo.

Agora, por argumentos usuais, podemos supor a existência de uma solução global $\xi^{(1)}: \mathbb{R} \rightarrow X$ para $T_{0}(\cdot)$ tal que, pondo $\xi_{r}^{(1)}(t):=\xi_{k_{r}}\left(t+t_{r}^{\prime}\right)$ com $t \in\left[-\left(t_{r}^{\prime}-t_{r}\right), \infty\right)$, tem-se $\xi_{r}^{(1)}(t) \underset{r \rightarrow \infty}{\rightarrow} \xi^{(1)}(t)$ uniformemente para $t$ em compactos da reta.

Pela maneira com a qual $\xi^{(1)}: \mathbb{R} \rightarrow X$ foi obtida devemos ter

$$
d\left(\xi^{(1)}(t), \Xi_{i_{0}, 0}\right) \leq \delta \text { sempre que } t \leq 0,
$$

e a propriedade $(G 1)$ do semigrupo de tipo gradiente $T_{0}(\cdot)$ em conjunto com a maneira com a qual $\delta_{0}$ foi escolhido, asseguram que, necessariamente,

$$
\lim _{t \rightarrow-\infty} d\left(\xi^{(1)}(t), \Xi_{i_{0}, 0}\right)=0
$$

e como $d\left(\xi^{(1)}(0), \Xi_{i_{0}, 0}\right)=\delta,(G 1)$ e $(G 2)$ obrigam que

$$
\lim _{t \rightarrow \infty} d\left(\xi^{(1)}(t), \Xi_{i_{1}, 0}\right)=0
$$

para um certo invariante isolado $\Xi_{i_{1}, 0} \in \boldsymbol{\Xi}_{0}$ com $i_{1} \neq i_{0}$.

Agora, como $\xi_{r}^{(1)}(t) \underset{r \rightarrow \infty}{\rightarrow} \xi^{(1)}(t)$ uniformemente para $t$ em compactos de $\mathbb{R}$, de (3.2.5) deduz-se que para cada $m \in \mathbb{N}\left(\operatorname{com} \frac{1}{m}<\delta\right)$ podemos encontrar $r_{m} \in \mathbb{N}$ e $t_{m} \in \mathbb{R}$ tais que

$$
d\left(\xi_{r}^{(1)}\left(t_{m}\right), \Xi_{i_{1}, 0}\right)<\frac{1}{m} \text { sempre que } r \geq r_{m}
$$

e de (3.2.3), analogamente ao caso anterior, sgue-se a existência de $t_{m}^{\prime}>t_{m}$ de modo que

$$
d\left(\xi_{r_{m}}^{(1)}(t), \Xi_{i_{1}, 0}\right)<\delta \text { para todo } t \in\left[t_{m}, t_{m}^{\prime}\right)
$$

$\mathrm{e}$

$$
d\left(\xi_{r_{m}}^{(1)}\left(t_{m}^{\prime}\right), \Xi_{i_{1}, 0}\right)=\delta .
$$

Como no caso anterior, temos $t_{m}^{\prime}-t_{m} \rightarrow \infty$ e podemos supor a existência de uma solução global $\xi^{(2)}: \mathbb{R} \rightarrow X$ para $T_{0}(\cdot)$, tal que, definindo $\xi_{m}^{(2)}(t):=\xi_{r_{m}}\left(t+t_{m}^{\prime}\right)$ com $t \in\left[-\left(t_{m}^{\prime}-t_{m}\right), \infty\right)$, segue-se que $\xi_{m}^{(2)}(t) \underset{m \rightarrow \infty}{\rightarrow} \xi^{(2)}(t)$ uniformemente para $t$ em compactos da reta.

Pela maneira com a qual se obtém $\xi^{(2)}: \mathbb{R} \rightarrow X$ devemos ter

$$
d\left(\xi^{(2)}(t), \Xi_{i_{1}, 0}\right) \leq \delta \text { sempre que } t \leq 0,
$$

e a propriedade $(G 1)$ que possui o semigrupo de tipo gradiente $T_{0}(\cdot)$ conjuntamente com a maneira com a qual $\delta_{0}$ foi escolhido, implicam que, necessariamente,

$$
\lim _{t \rightarrow-\infty} d\left(\xi^{(2)}(t), \Xi_{i_{1}, 0}\right)=0
$$


e sendo $d\left(\xi^{(2)}(0), \Xi_{i_{1}, 0}\right)=\delta,(G 1)$ e $(G 2)$ obrigam que

$$
\lim _{t \rightarrow \infty} d\left(\xi^{(2)}(t), \Xi_{i_{2}, 0}\right)=0
$$

para algum invariante isolado $\Xi_{i_{2}, 0} \in \boldsymbol{\Xi}_{0}$ com $i_{2} \neq i_{1}$.

Observemos agora que, se ocorresse que $i_{2}=i_{0}$, haveríamos obtido uma estrutura homoclínica no atrator do semigrupo de tipo gradiente $T_{0}(\cdot)$ associada a $\boldsymbol{\Xi}_{0}$, o que evidentemente não é possível. Logo, podemos repetir todo o raciocínio que realizamos acima, o qual deverá parar após um número finito de etapas, pois o conjunto $\boldsymbol{\Xi}_{0}$ é finito e, como vimos, no último passo nos veremos obrigados a encontrar uma estrutura homoclínica no atrator de $T_{0}(\cdot)$ associada a $\boldsymbol{\Xi}_{0}$, o que é uma contradição e assim nossa afirmação inicial é correta.

De manera inteiramente análoga a que fizemos acima, simplesmente trocando o sentido para onde se move o tempo, podemos provar a seguinte afirmação:

Existe $\eta_{2} \in\left(0, \eta_{1}\right]$ (onde $\eta_{1}$ cumpre o que acabamos de provar na análise anterior) tal que para $\eta \in\left[0, \eta_{2}\right]$ se $\xi_{\eta}: \mathbb{R} \rightarrow X$ é solução global para $T_{\eta}(\cdot)$, então existe $t_{\eta} \in \mathbb{R}$ de modo que

$$
\sup _{t \leq t_{\eta}} d\left(\xi_{\eta}(t), \bigcup_{i=1}^{n} \Xi_{i, 0}\right)<\delta,
$$

onde $\delta>0$ se obtém, como antes, a partir da hipótese (b), então, da maximalidade dos invariantes, conclui-se que

$$
\lim _{t \rightarrow-\infty} d\left(\xi_{\eta}(t), \bigcup_{i=1}^{n} \Xi_{i, \eta}\right)=0
$$

o que irá estabelecer, junto com o caso anterior, a estabilidade de $(G 1)$ terminando a primeira parte da demonstração.

Agora, provemos que existe $\eta_{0} \in\left(0, \eta_{2}\right]\left(\eta_{2}>0\right.$ sendo tal que a estabilidade de $(G 1)$ verifica-se, pelo que provamos anteriormente) tal que para todo $\eta \in\left[0, \eta_{0}\right]$ o atrator do semigrupo $T_{\eta}(\cdot)$ não possui estruturas homoclínicas associada a $\boldsymbol{\Xi}_{\eta}$.

De fato, porque caso contrário, usando o fato de que os conjuntos $\boldsymbol{\Xi}_{\eta}$ são todos finitos possuindo o mesmo número de elementos, conclui-se a existência de uma sequência $\eta_{k} \rightarrow 0^{+}$, uma sequência $\left(\boldsymbol{\Xi}_{k}^{\prime}\right)_{k \in \mathbb{N}}$ de coleções de conjuntos com $\boldsymbol{\Xi}_{k}^{\prime}:=$ $\left\{\Xi_{l_{1}, \eta_{k}}, \Xi_{l_{2}, \eta_{k}}, \cdots, \Xi_{l_{p}, \eta_{k}}\right\} \subset \boldsymbol{\Xi}_{\eta_{k}}$ para todo natural $k$, e uma sequência de conjuntos de soluções $\left\{\xi_{k, j}: \mathbb{R} \rightarrow X: j=1,2, \cdots, p\right\}_{k \in \mathbb{N}}$ tais que para cada natural $k$ e cada $j=1,2, \cdots, p, \xi_{k, j}$ é solução global de $T_{\eta_{k}}(\cdot)$ satisfazendo, para cada natural $k$ e $j=1,2, \cdots, p$,

$$
\lim _{t \rightarrow-\infty} d\left(\xi_{k, j}(t), \Xi_{l_{j}, \eta_{k}}\right)=0 \text { e } \lim _{t \rightarrow \infty} d\left(\xi_{k, j}(t), \Xi_{l_{(j+1)}, \eta_{k}}\right)=0,
$$


onde para cada $k$ colocamos $\Xi_{l_{(p+1)}, \eta_{k}}:=\Xi_{l_{1}, \eta_{k}}$.

Passando a uma subsequência, se necessário, podemos supor que para cada natural $k$ e cada $j=1,2, \cdots, p$ existe um real $t_{k}^{(j)}$ de maneira que

$$
d\left(\xi_{k, j}\left(t_{k}^{(j)}\right), \Xi_{l_{j}, 0}\right)<\frac{1}{k}
$$

e como podemos supor $\Xi_{l_{j}, \eta_{k}} \neq \Xi_{l_{(j+1)}, \eta_{k}}$ para todo $k$ e todo $j$, vemos que existe também $t_{k}^{(j) \prime}>t_{k}^{(j)}$ de modo que

$$
d\left(\xi_{k, j}(t), \Xi_{l_{j}, 0}\right)<\delta \text { toda vez que } t \in\left[t_{k}^{(j)}, t_{k}^{(j) \prime}\right)
$$

e

$$
d\left(\xi_{k, j}\left(t_{k}^{(j) \prime}\right), \Xi_{l_{j}, 0}\right)=\delta .
$$

E assim vemos que, desta forma, acabamos de criar todas as condições necessárias para podermos repetir o mesmo argumento que usamos para concluir a estabilidade de $(G 1)$ e com isto o teorema fica demonstrado. 


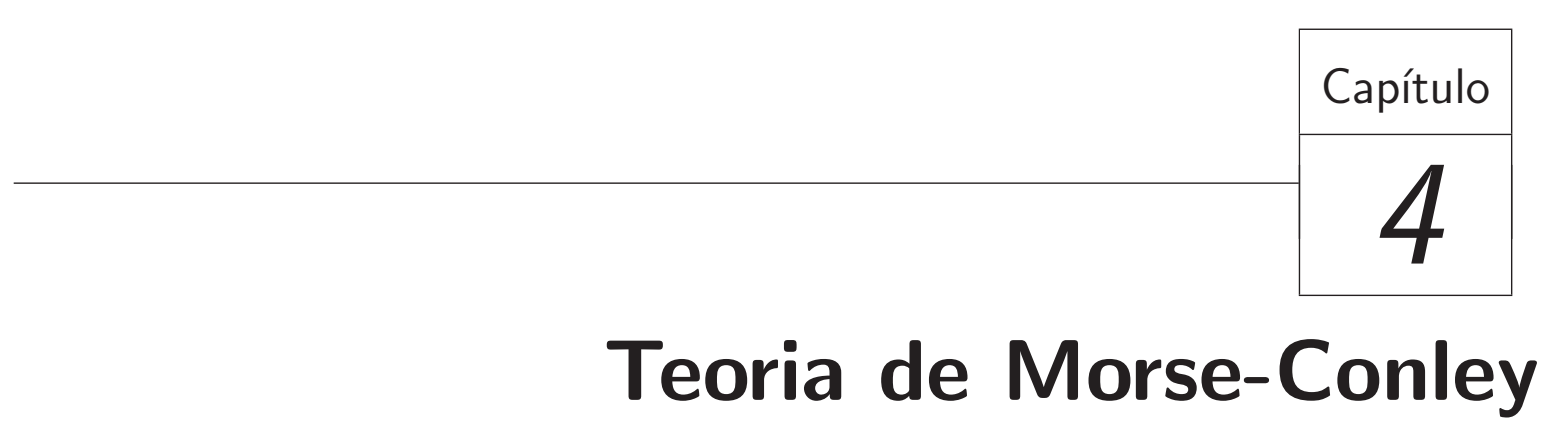

Neste capítulo demonstramos a equivalência entre os dois conceitos que estudamos nos dois últimos capítulos, isto é, que as noções de semigrupo gradiente e de semigrupo de tipo gradiente coincidem. Então, como uma consequência do Teorema 3.2.4, obteremos que o conceito de semigrupo gradiente é estável por perturbação, um resultado desconhecido até o momento.

Além disso, forneceremos condições que assegurem que as funções de Lyapunov correspondentes sejam também estáveis por perturbação.

Para levar a cabo esta tarefa introduzimos alguns aspectos da teoria de Morse, inspirados nos conceitos que se encontram em [12].

\subsection{Pares atrator-repulsor}

A noção de atrator local e repulsor complementar, que a seguir definimos, é o elemento decisivo que nos permite construir as funções de Lyapunov para os semigrupos de tipo gradiente.

Definição 4.1.1. Seja $\{T(t): t \geq 0\}$ um semigrupo em um espaço métrico $X$ possuindo atrator global $\mathcal{A}$. Diz-se que um subconjunto $A \subset \mathcal{A}$ é um atrator local para $T(\cdot)$ quando existe $\varepsilon>0$ de maneira que $\omega\left(\mathcal{O}_{\varepsilon}(A)\right)=A$.

Se $A \subset \mathcal{A}$ é um atrator local, seu repulsor complementar, indicado por $A^{*}$, é o conjunto definido por

$$
A^{*}:=\{x \in \mathcal{A}: \omega(x) \cap A=\varnothing\} .
$$

Nestas condições, o par $\left(A, A^{*}\right)$ chama-se um par atrator-repulsor. 
Se $\left(A, A^{*}\right)$ é um par atrator-repulsor, então $A$ e $A^{*}$ são disjuntos.

Observemos que, quando $A$ é um atrator local, então seu repulsor complementar, $A^{*}$, é também fechado e invariante. Com efeito, seja $\left(x_{n}\right)_{n \in \mathbb{N}}$ uma sequência de pontos de $A^{*}$ convergindo para um ponto $x \in X$. Claramente $x \in \mathcal{A}$, porque $\mathcal{A}$ é fechado, então se $x$ não pertencesse a $A^{*}$, por definição, existiria um ponto $z \in \omega(x) \cap A$, portanto existiria $\left(t_{n}\right)_{n \in \mathbb{N}}$, uma sequência de números positivos, com $t_{n} \rightarrow \infty$ de modo que $z=\lim _{n \rightarrow \infty} T\left(t_{n}\right) x$. Então, escolhendo $\varepsilon>0$ tal que $\omega\left(\mathcal{O}_{\varepsilon}(A)\right)=A$ e $N \in \mathbb{N}$ tal que $T\left(t_{N}\right) x \stackrel{n \rightarrow \infty}{\in} \mathcal{O}_{\varepsilon}(A)$, a continuidade do operador $T\left(t_{N}\right): X \rightarrow X$ forneceria a existência de um natural $n_{0}$ de forma que $T\left(t_{N}\right) x_{n} \in \mathcal{O}_{\varepsilon}(A)$ para todo $n \geq n_{0}$, logo

$$
\omega\left(x_{n}\right)=\omega\left(T\left(t_{N}\right) x_{n}\right) \subset \omega\left(\mathcal{O}_{\varepsilon}(A)\right)=A \text { para todo } n \geq n_{0},
$$

o que contrariaria o fato de que $x_{n} \in A^{*}$ para todo natural $n$, mostrando que $A^{*}$ é fechado.

Para ver a invariância de $A^{*}$, sejam $x \in A^{*}$ e $t \geq 0$ quaisquer, então, por um lado, $\omega(x) \cap A=\varnothing$, donde vem, do fato de que $\omega(x)=\omega(T(t) x)$, que $\omega(T(t) x) \cap A=\varnothing$, o que significa que $T(t) x \in A^{*}$. Reciprocamente, dado $t \geq 0$, seja $z \in A^{*}$, então, pela invariância do atrator global $\mathcal{A}$, $z$ é da forma $z=T(t) x$ para algum $x \in \mathcal{A}$ e pela mesma razão de antes, $\omega(x)=\omega(z)$, portanto, $x \in A^{*}$ e assim $z \in T(t) A^{*}$, como queríamos.

Observemos também, que $A$ é um atrator local se, e somente se, é um conjunto compacto invariante atraindo $\mathcal{O}_{\varepsilon}(A)$ para algum $\varepsilon>0$. De fato, se $A$ é um atrator local, então, pela própria definição, existe $\varepsilon>0$ tal que $A=\omega\left(\mathcal{O}_{\varepsilon}(A)\right)$ e assim, $A$ é compacto invariante atraindo $\mathcal{O}_{\varepsilon}(A)$, simplesmente como consequência das conclusões do Lema 1.2.6. Reciprocamente, suponhamos que $A$ é compacto invariante atraindo $\mathcal{O}_{\varepsilon}(A)$, para algum $\varepsilon>0$, daí, dado $\delta>0$ segue a existência de $t(\delta)>0$ tal que

$$
T(t) \mathcal{O}_{\varepsilon}(A) \subset \mathcal{O}_{\delta}(A) \text { sempre que } t \geq t(\delta),
$$

donde vemos que $\omega\left(\mathcal{O}_{\varepsilon}(A)\right) \subset \overline{\mathcal{O}_{\delta}(A)}$, para todo $\delta>0$, e por isso

$$
\omega\left(\mathcal{O}_{\varepsilon}(A)\right) \subset \bigcap_{\delta>0} \overline{\mathcal{O}_{\delta}(A)}
$$

mas, sendo $A$ um conjunto fechado, é fácil comprovar que $\bigcap_{\delta>0} \overline{\mathcal{O}_{\delta}(A)}=A$, o que implica $\omega\left(\mathcal{O}_{\varepsilon}(A)\right) \subset A$. Agora, como $A$ é fechado invariante temos, pelo Lema 1.2.8, que $A=\omega(A)$, e como $A \subset \mathcal{O}_{\varepsilon}(A)$ temos $\omega(A) \subset \omega\left(\mathcal{O}_{\varepsilon}(A)\right)$, completando a explicação.

Do que analisamos acima segue, evidentemente, que um atrator global é também um atrator local. 
Devemos observar que nossa definição de atrator local é ligeiramente diferente da que se encontra em [12], uma vez que aqui consideramos, como vizinhanças do conjunto $A$ cujo $\omega$-limite é o próprio $A$, abertos legítimos do espaço $X$ e não somente restritos ao atrator $\mathcal{A}$, como se estuda em [12] e [20], onde o espaço de fase é suposto compacto. Entretanto, mostraremos a seguir que nossa definição, de certa forma, coincide com a dada em [12].

O interesse em se considerar semigrupos em espaços de fases não compactos, reside no fato de que, desta forma, obtem-se conclusões da teoria de Morse que, em termos práticos, podem ser aplicadas ao estudo da dinâmica de EDP's, razão que motivou o início deste trabalho.

Para provarmos a equivalência entre nossa definição de atrator local e a dada em [12], o seguinte lema é um resultado chave, de fato, ele será crucial para todos os resultados que desenvolveremos daqui em diante no contexto dos sistemas dinâmicos autônomos.

Também, como havíamos dito antes, aqui não trabalhamos com a hipótese de que cada $T(t): X \rightarrow X$ é um homeomorfismo, o que ocorre em [12], por exemplo. Nesta direção, nosso próximo lema nos ajuda a contornar a ausência desta propriedade além de melhorar o item $a$ ) da Proposição 1.3 em [20].

Lema 4.1.2. Seja $\{T(t): t \geq 0\}$ um semigrupo num espaço métrico $X$ possuindo atrator global $\mathcal{A}$.

Se $A$ é um compacto invariante por $T(\cdot)$ e existe $\varepsilon>0$ tal que $A$ atrai o conjunto $\mathcal{A} \cap \mathcal{O}_{\varepsilon}(A)$ por meio de $T(\cdot)$, então dado $\delta \in(0, \varepsilon)$ existe $\delta^{\prime} \in(0, \delta)$ de modo que

$$
\gamma^{+}\left(\mathcal{O}_{\delta^{\prime}}(A)\right) \subset \mathcal{O}_{\delta}(A)
$$

Demonstração: Com efeito, suponhamos que esta conclusão não se verifique, então existem $\delta \in(0, \varepsilon)$ e sequências $\left(x_{j}\right)_{j \in \mathbb{N}}$, de pontos de $X$, e $\left(t_{j}\right)_{j \in \mathbb{N}}$ em $\mathbb{R}_{0}^{+}$, tais que $t_{j} \underset{j \rightarrow \infty}{\rightarrow} \infty$

$$
\begin{gathered}
d\left(x_{j}, A\right)<\frac{1}{j} \text { para todo } j, \\
d\left(T(t) x_{j}, A\right)<\delta \text { para todo } t \in\left[0, t_{j}\right) \text { e todo } j \in \mathbb{N}
\end{gathered}
$$

e

$$
d\left(T\left(t_{j}\right) x_{j}, A\right)=\delta \text { para todo } j \in \mathbb{N} .
$$

Agora, definindo, para cada natural $j, \xi_{j}:\left[-t_{j}, \infty\right) \rightarrow X$ por $\xi_{j}(t):=T\left(t+t_{j}\right) x_{j}$, usando as hipóteses de compacidade assintótica coletiva e de convergência uniforme em compactos para a família constante de semigrupos $\left(T_{\eta}\right)_{\eta \in[0,1]}, T_{\eta}=T$ para todo $\eta$, pelo Lema 3.2.3, existe uma solução global limitada $\xi_{0}: \mathbb{R} \rightarrow X$ para o semigrupo 
$\{T(t): t \geq 0\}$ e uma subsequência de $\left(\xi_{j}\right)_{j \in \mathbb{N}}$, que denotamos da mesma maneira, tal que para todo real $t, \xi_{0}(t)=\lim _{j \rightarrow \infty} \xi_{j}(t)$.

Por outro lado, dado $t<0$ para $j$ suficientemente grande temos

$$
d\left(\xi_{j}(t), A\right)<\delta
$$

donde conclui-se, após fazer $j \rightarrow \infty$, que para todo $t<0$

$$
d\left(\xi_{0}(t), A\right) \leq \delta
$$

e do fato de que $\delta=d\left(\xi_{j}(0), A\right)$ obtém-se $d\left(\xi_{0}(0), A\right)=\delta$.

Finalmente, como $\delta<\varepsilon$, obrigatoriamente $A$ atrai $K:=\left\{\xi_{0}(t): t \leq 0\right\} \subset \mathcal{A} \cap$ $\mathcal{O}_{\varepsilon}(A)$, e por isso, dado $\mu \in(0, \delta)$ existe um $t_{0}>0$ tal que $d(T(t) z, A)<\mu$ para todo $z \in K$ sempre que $t \geq t_{0}$, mas, em particular, para $z:=\xi_{0}\left(-t_{0}\right) \in K$ vem que

$$
\mu>d\left(T\left(t_{0}\right) z, A\right)=d\left(T\left(t_{0}\right) \xi_{0}\left(-t_{0}\right), A\right)=d\left(\xi_{0}(0), A\right)=\delta
$$

contradizendo $\mu<\delta$ e completando a prova.

Obviamente, se $A \subset \mathcal{A}$ é um atrator local para $T(\cdot)$ segundo a Definição 4.1.1, então $A$ satisfaz as hipóteses do resultado anterior e mais geralmente tem-se a

Observação 4.1.3. Se um invariante isolado limitado $A$ tem conjunto instável trivial, então ele satisfaz a conclusão do lema anterior, ou seja, se $A$ é um invariante isolado limitado com $W^{u}(A)=A$, então dado $\delta \in(0, \varepsilon)$ existe $\delta^{\prime} \in(0, \delta)$ tal que

$$
\gamma^{+}\left(\mathcal{O}_{\delta^{\prime}}(A)\right) \subset \mathcal{O}_{\delta}(A)
$$

Com efeito, basta repetir o início da demonstração do lema anterior e lembrar que o conjunto $\alpha$-limite de uma solução global limitada é invariante e atrai a solução quando $t \rightarrow-\infty$.

Provemos, no próximo resultado, a equivalência entre nossa definição de atrator local e a dada en [12].

Lema 4.1.4. Seja $\{T(t): t \geq 0\}$ um semigrupo em um espaço métrico $X$ possuindo atrator global $\mathcal{A}$. Seja também, para cada $t \geq 0, S(t):=\left.T(t)\right|_{\mathcal{A}}$. Graças à invariância de $\mathcal{A},\{S(t): t \geq 0\}$ resulta um semigrupo no espaço métrico $\mathcal{A}$.

Tem-se:

(i) Se $A \subset \mathcal{A}$ é um atrator local para $\{S(t): t \geq 0\}$ em $\mathcal{A}$ e $K \subset \mathcal{A}$ é um compacto disjunto de $A^{*}$, então $A$ atrai $K$ por $S(\cdot)$. 
(ii) Se $A \subset \mathcal{A}$ é um atrator local para $\{S(t): t \geq 0\}$ em $\mathcal{A}$, então também o é para $\{T(t): t \geq 0\}$ em $X$.

Demonstração: ( $i$ ) Seja $K$ um subconjunto compacto de $\mathcal{A}$ tal que $K \cap A^{*}=\varnothing$. Se $A$ não atraisse a $K$, existiria $\delta>0$ e uma sequência de números não negativos $\left(t_{j}\right)_{j \in \mathbb{N}}$, com $t_{j} \rightarrow \infty$, de maneira que

$$
\operatorname{dist}\left(S\left(t_{j}\right) K, A\right) \geq 2 \delta \text { para todo } j \in \mathbb{N},
$$

e por isto, existiria uma sequência $\left(x_{j}\right)_{j \in \mathbb{N}}$ de pontos de $K$ satisfazendo

$$
d\left(S\left(t_{j}\right) x_{j}, A\right) \geq \delta \text { para todo } j \in \mathbb{N} .
$$
$K$.

Podemos supor, passando a uma subsequência caso seja necessário, que $x_{j} \underset{j \rightarrow \infty}{\rightarrow} x \in$

Agora, como o conjunto $A$ satisfaz as hipóteses do lema anterior, seja $\delta^{\prime} \in(0, \delta)$ de modo que

$$
\gamma^{+}\left(\mathcal{O}_{\delta^{\prime}}(A)\right) \subset \mathcal{O}_{\delta}(A),
$$

logo, obrigatoriamente, $d\left(S(t) x_{j}, A\right) \geq \delta^{\prime}$ para todo $t \in\left[0, t_{j}\right]$ e todo $j \in \mathbb{N}$. Portanto, como $t_{j} \underset{j \rightarrow \infty}{\rightarrow} \infty$ e $x_{j} \underset{j \rightarrow \infty}{\rightarrow} x$, segue-se que para todo $t>0$

$$
d(S(t) x, A) \geq \delta^{\prime}
$$

A desigualdade anterior nos diz que $d(\omega(x), A) \geq \delta^{\prime}$, donde $\omega(x) \cap A=\varnothing$, e como $x \in K \subset \mathcal{A}$, resulta $x \in A^{*}$, contradizendo $K \cap A^{*}=\varnothing$ e completando a prova do item $(i)$.

(ii) Sendo $A$ e $A^{*}$ compactos disjuntos, existe $\delta>0$, suficientemente pequeno, de modo que $\mathcal{O}_{2 \delta}(A) \cap A^{*}=\varnothing$. Logo, usando o Lema 4.1.2, obtém-se a existência de $\delta^{\prime} \in(0, \delta)$ de maneira que

$$
\gamma^{+}\left(\mathcal{O}_{\delta^{\prime}}(A)\right) \subset \mathcal{O}_{\delta}(A),
$$

o que nos dá $\omega\left(\mathcal{O}_{\delta^{\prime}}(A)\right) \subset \overline{\mathcal{O}_{\delta}(A)} \cap \mathcal{A}$ e, portanto, $\omega\left(\mathcal{O}_{\delta^{\prime}}(A)\right) \cap A^{*}=\varnothing$.

Agora, aplicando a conclusão do item anterior deduz-se que $A$ atrai $\omega\left(\mathcal{O}_{\delta^{\prime}}(A)\right)$ por $S(\cdot)$ (e portanto por $T(\cdot)$ ), e como $A$ é fechado, da invariância de $\omega\left(\mathcal{O}_{\delta^{\prime}}(A)\right)$ por $T(\cdot)$ conclui-se que $\omega\left(\mathcal{O}_{\delta^{\prime}}(A)\right) \subset A$, por outro lado, o fato de que $A \subset \mathcal{O}_{\delta^{\prime}}(A)$ e o Lema 1.2.8 implicam que $A \subset \omega\left(\mathcal{O}_{\delta^{\prime}}(A)\right)$, ou seja, $A=\omega\left(\mathcal{O}_{\delta^{\prime}}(A)\right)$, mostrando que $A$ é também um atrator local para $T(\cdot)$ em $X$, como queríamos.

Nossas próximas considerações têm por objetivo estabelecer que, se $\left(A, A^{*}\right)$ é um par atrator-repulsor para $T(\cdot)$, então $T(\cdot)$ é um semigrupo de tipo gradiente com respeito à família $\boldsymbol{\Xi}:=\left\{A, A^{*}\right\}$. Para isso serão úteis os três resultados auxiliares seguintes: 
Lema 4.1.5. Sejam $\left(A, A^{*}\right)$ um par atrator-repulsor e $\xi: \mathbb{R} \rightarrow X$ uma solução global para um semigrupo $\{T(t): t \geq 0\}$ em um espaço métrico $X$ possuindo atrator global $\mathcal{A}$. Se existe $t_{0} \in \mathbb{R}$ de maneira que

$$
d\left(\xi(t), A^{*}\right)<\delta \text { para todo } t \leq t_{0},
$$

para um certo $\delta>0$ com $\mathcal{O}_{\delta}\left(A^{*}\right) \cap \mathcal{O}_{\delta}(A)=\varnothing$, então

$$
\lim _{t \rightarrow-\infty} d\left(\xi(t), A^{*}\right)=0
$$

Demonstração: Se a conclusão não fosse certa, existiriam $\varepsilon>0$ e uma sequência de números positivos $\left(t_{j}\right)_{j \in \mathbb{N}}, \operatorname{com} t_{j} \rightarrow \infty$, de modo que para todo natural $j$

$$
d\left(\xi\left(-t_{j}\right), A^{*}\right) \geq \varepsilon .
$$

Assim, o conjunto $\left\{\xi\left(-t_{j}\right): j \in \mathbb{N}\right\}$ estaria contido no compacto $K:=\{x \in \mathcal{A}$ : $\left.d\left(x, A^{*}\right) \geq \varepsilon\right\}$ que, pelo lema anterior, deve ser atraído por $A$, logo existiria $\tau>0$ com

$$
d(T(t) z, A)<\delta \text {, para todo } z \in K \text { e todo } t \geq \tau \text {. }
$$

Então, em particular, escolhendo $z:=\xi\left(-t_{j_{0}}\right) \in K$, onde $t_{j_{0}} \geq \tau-t_{0}$, teríamos

$$
d\left(\xi\left(t_{0}\right), A\right)=d\left(T\left(t_{j_{0}}+t_{0}\right) \xi\left(-t_{j_{0}}\right), A\right)=d\left(T\left(t_{j_{0}}+t_{0}\right) z, A\right)<\delta,
$$

o que contrariaria a escolha de $\delta$.

O resultado seguinte é uma adaptação de seu análogo encontrado em [20] (item (b) da Proposição 1.3).

Lema 4.1.6. Sejam $\left(A, A^{*}\right)$ um par atrator-repulsor para um semigrupo $\{T(t): t \geq 0\}$ num espaço métrico $X$ possuindo atrator global $\mathcal{A}$ e $F \subset \mathcal{A}$ um subconjunto fechado com $F \cap A=\varnothing$.

Então, dado $\varepsilon>0$ existe $t_{\varepsilon}>0$ tal que $d\left(x, A^{*}\right)<\varepsilon$ toda vez que $T(t) x \in F$ com $t \geq t_{\varepsilon}$ e $x \in \mathcal{A}$.

Demonstração: Com efeito, caso contrário, existem $\varepsilon>0$, uma sequência $\left(x_{j}\right)_{j \in \mathbb{N}}$ de pontos de $\mathcal{A}$ e uma sequência de números reais positivos $\left(t_{j}\right)_{j \in \mathbb{N}}$, com $t_{j} \rightarrow \infty$, tais que $T\left(t_{j}\right) x_{j} \in F$ mas $d\left(x_{j}, A^{*}\right) \geq \varepsilon$.

Em virtude da compacidade de $\mathcal{A}$ podemos supor que $x_{j} \underset{j \rightarrow \infty}{\rightarrow} x \in \mathcal{A}$ e, como $d\left(x, A^{*}\right) \geq \varepsilon$, devemos ter $\omega(x) \cap A \neq \varnothing$. Então, aplicando o Lema 4.1.2 é fácil ver que, dado $0<\delta<d(F, A)$ existem $\delta^{\prime} \in(0, \delta)$ e $\tau_{1}>0$ de modo que

$$
T\left(\tau_{1}\right) x \in \mathcal{O}_{\delta^{\prime}}(A)
$$


sendo que

$$
\gamma^{+}\left(\mathcal{O}_{\delta^{\prime}}(A)\right) \subset \mathcal{O}_{\delta}(A) .
$$

Pela continuidade de $T\left(\tau_{1}\right): X \rightarrow X$, existe um natural $j_{0}$ de maneira que $T\left(\tau_{1}\right) x_{j} \in \mathcal{O}_{\delta^{\prime}}(A)$ para todo $j \geq j_{0}$, logo, por (4.1.1), $T\left(t+\tau_{1}\right) x_{j} \in \mathcal{O}_{\delta}(A)$ para todo $t \geq 0$ e todo $j \geq j_{0}$ e, em particular, se $t_{j}>\tau_{1}$ com $j \geq j_{0}$ temos

$$
T\left(t_{j}\right) x_{j}=T\left(\left(t_{j}-\tau_{1}\right)+\tau_{1}\right) x_{j} \in \mathcal{O}_{\delta}(A),
$$

contradizendo que $T\left(t_{j}\right) x_{j} \in F$ dada a escolha do $\delta$, terminando assim a demonstração.

Lema 4.1.7. Seja $\left(A, A^{*}\right)$ um par atrator-repulsor para um semigrupo $\{T(t): t \geq 0\}$ em um espaço métrico $X$ possuindo atrator global $\mathcal{A}$.

Verificam-se as seguintes propriedades:

(i) $S e \xi: \mathbb{R} \rightarrow X$ é uma solução global limitada para $T(\cdot) \operatorname{com} \xi(0)=: x \notin\left(A \cup A^{*}\right)$, então

$$
\lim _{t \rightarrow-\infty} d\left(\xi(t), A^{*}\right)=0 e \lim _{t \rightarrow \infty} d(\xi(t), A)=0 .
$$

(ii) Para todo $x \in X$ tem-se que, ou $\lim _{t \rightarrow \infty} d(T(t) x, A)=0$ ou $\lim _{t \rightarrow \infty} d\left(T(t) x, A^{*}\right)=0$.

Demonstração: $(i)$ Com efeito, em primeiro lugar, como $x \notin A^{*}$, do item $(i)$ do Lema 4.1.4 deduz-se que $\lim _{t \rightarrow \infty} d(T(t) x, A)=0$, o que significa o mesmo que $\lim _{t \rightarrow \infty} d(\xi(t), A)=$ 0 .

Em segundo, pondo $F:=\{x\}$, vemos que $F \cap A=\varnothing$ e portanto, pelo lema anterior, dado $\varepsilon>0$ existe $t_{\varepsilon}>0$ tal que $d\left(z, A^{*}\right)<\varepsilon$ sempre que $T(t) z \in F$ com $t \geq t_{\varepsilon}$ e $z \in \mathcal{A}$, ou seja, sempre que $T(t) z=x \operatorname{com} z \in \mathcal{A}$ e $t \geq t_{\varepsilon}$. Em particular, para $t \leq-t_{\varepsilon}$, como $\xi(t) \in \mathcal{A}$ e $T(-t) \xi(t)=\xi(0)=x$ com $-t \geq t_{\varepsilon}$ segue-se $d\left(\xi(t), A^{*}\right)<\varepsilon$, donde conclui-se que $\lim _{t \rightarrow-\infty} d\left(\xi(t), A^{*}\right)=0$, estabelecendo o item $(i)$.

(ii) O caso em que $x \in \mathcal{A}$ segue do item anterior e da invariância dos conjuntos fechados $A$ e $A^{*}$. Suponhamos então que seja $x \notin \mathcal{A}$. Neste caso, sejam $0<\delta^{\prime}<\delta$ satisfazendo a conclusão do Lema 4.1.2, isto é,

$$
\gamma^{+}\left(\mathcal{O}_{\delta^{\prime}}(A)\right) \subset \mathcal{O}_{\delta}(A)
$$

com $\delta>0$ satisfazendo $\omega\left(\mathcal{O}_{\delta}(A)\right)=A$. Então, por um lado, se existe $t_{0}>0$ tal que $T\left(t_{0}\right) x \in \mathcal{O}_{\delta^{\prime}}(A)$, obrigatoriamente, $T(t) x \underset{t \rightarrow \infty}{\rightarrow} A$.

Por outro, se para todo $t>0$

$$
d(T(t) x, A) \geq \delta^{\prime}
$$


então $T(t) x \underset{t \rightarrow \infty}{\rightarrow} A^{*}$, porque de outro modo, existiria uma sequência de números positivos $\left(t_{j}\right)_{j \in \mathbb{N}}$, com $t_{j} \rightarrow \infty$, e um $\varepsilon>0$ tais que

$$
d\left(T\left(t_{j}\right) x, A^{*}\right) \geq \varepsilon \text { para todo } j \in \mathbb{N} .
$$

Agora, definindo para cada natural $j, \xi_{j}:\left[-t_{j}, \infty\right) \rightarrow X$ por $\xi_{j}(t):=T\left(t+t_{j}\right) x$, por aplicação do Lema 3.2.3, concluiríamos a existência de uma solução global $\xi_{0}$ : $\mathbb{R} \rightarrow X$ para $T(\cdot)$ tal que, poderiamos supor, $\xi_{0}(t)=\lim \xi_{j}(t)$ para todo real $t$. Donde seguiria de (4.1.3) que $d\left(\xi_{0}(0), A^{*}\right) \geq \varepsilon$, e graças ao Lema 4.1.4, dado que $\xi_{0}(0) \in \mathcal{A}$, deduziríamos que $\xi_{0}(t)=T(t) \xi_{0}(0) \underset{t \rightarrow \infty}{\rightarrow} A$. Mas (4.1.2) implicaria que para todo real $t, d\left(\xi_{0}(t), A\right) \geq \delta^{\prime}$, impedindo que a convergência $\xi_{0}(t) \underset{t \rightarrow \infty}{\rightarrow} A$ se satisfizesse e terminando a prova.

A propriedade dada em (ii) acima, não encontrada em [12] ou [20], desempenha um papel importante na prova da continuidade da "função de Lyapunov" em pontos de $X$ que não estão no atrator, como veremos na próxima seção.

Finalmente, unindo os três lemas anteriores obtém-se a seguinte:

Proposição 4.1.8. Seja $\left(A, A^{*}\right)$ um par atrator-repulsor para um semigrupo $\{T(t)$ : $t \geq 0\}$ em um espaço métrico $X$ possuindo atrator global $\mathcal{A}$. Então, $\boldsymbol{\Xi}:=\left\{A, A^{*}\right\}$ é uma família disjunta de conjuntos invariantes isolados tal que $\{T(t): t \geq 0\}$ é um semigrupo de tipo gradiente com respeito a ela.

Demonstração: Para ver que $\boldsymbol{\Xi}:=\left\{A, A^{*}\right\}$ é uma família disjunta de conjuntos invariantes isolados, em primeiro lugar, escolhemos $\delta>0$ de maneira que $\mathcal{O}_{\delta}\left(A^{*}\right) \cap$ $\mathcal{O}_{\delta}(A)=\varnothing$ e que $\omega\left(\mathcal{O}_{\delta}(A)\right)=A$.

Agora, por um lado, se $\Xi \subset \mathcal{O}_{\delta}(A)$ é um conjunto invariante, como $A$, em virtude do Lema 1.2.6, atrai $\mathcal{O}_{\delta}(A)$, deve também atrair $\Xi$, mas a invariância de $\Xi$ obriga $\Xi$ a estar contido em $A$, mostrando que $A$ é o conjunto invariante maximal em $\mathcal{O}_{\delta}(A)$, que em outras palavras, significa que $A$ é um invariante isolado.

Por outro, seja $\Xi \subset \mathcal{O}_{\delta}\left(A^{*}\right)$ um conjunto invariante. Se existe um ponto $x \in \Xi \backslash A^{*}$, como $\Xi \subset \mathcal{A}$, segue-se do Lema 4.1 .4 que $\lim _{t \rightarrow \infty} d(T(t) x, A)=0$, mas isto é impossível, levando em conta que $T(t) x \in \Xi \subset \mathcal{O}_{\delta}^{t \rightarrow \infty}\left(A^{*}\right)$ para todo $t \geq 0$, e $\delta>0$ é tal que $\mathcal{O}_{\delta}\left(A^{*}\right) \cap \mathcal{O}_{\delta}(A)=\varnothing$.

Para ver que $\{T(t): t \geq 0\}$ é um semigrupo de tipo gradiente com respeito a $\boldsymbol{\Xi}=$ $\left\{A, A^{*}\right\}$, basta aplicar a conclusão do item $(i)$ do Lema 4.1.7 para estabelecer tanto a propriedade $(G 1)$ quanto $(G 2)$ da Definição 3.1.1, completando a prova. 


\subsection{Função de Lyapunov para um par atrator-repulsor}

Nesta seção provamos o resultado central para a construção de uma função de Lyapunov para um semigrupo de tipo gradiente. Tal resultado assegura a existência de uma função contínua, com propriedades bastante particulares, associada a um par atrator-repulsor. A demonstração que daremos, ainda que esteja fortemente inspirada na que se encontra em [12], é distinta por várias razões. Estas distinções, em geral, se originam no fato de que não trabalhamos, necessariamente, com grupos em espaços de fases compactos.

Por outro lado, ainda que em [20] todas as propriedades das decomposições de Morse estejam apresentadas para semigrupos, lá não se encontra uma construção de qualquer tipo de função de Lyapunov para um par atrator-repulsor de um semigrupo.

Para cumprir nosso objetivo, é necessário introduzir os efeitos da função dada no lema a seguir.

Lema 4.2.1. Seja $\{T(t): t \geq 0\}$ um semigrupo em um espaço métrico $X:=(X, d)$ possuindo atrator global $\mathcal{A}$. A função $h: X \rightarrow \mathbb{R}$ definida por

$$
h(x):=\sup _{t \geq 0} d(T(t) x, \mathcal{A}), x \in X,
$$

está bem definida, é contínua e não crescente ao longo de soluções de $T(\cdot) \operatorname{com} h(x)=0$ se, e somente se, $x \in \mathcal{A}$.

Demonstração: Com efeito, como para todo $x \in X$ existe $t_{x}>0$ tal que $T(t) x \in$ $\mathcal{O}_{1}(\mathcal{A})$ sempre que $t \geq t_{x}$ e a função real $\left[0, t_{x}\right] \ni t \longmapsto d(T(t) x, \mathcal{A}) \in \mathbb{R}$ é contínua definida em um compacto, segue-se que a função $h$ está bem definida, ou seja, assume valores reais em todos os pontos de $X$.

Por outro lado, dado $\varepsilon>0$ seja $0<\varepsilon^{\prime}<\varepsilon$ tal que $\gamma^{+}\left(\mathcal{O}_{\varepsilon^{\prime}}(\mathcal{A})\right) \subset \mathcal{O}_{\varepsilon}(\mathcal{A})$, o que prova a continuidade de $h$ nos pontos de $\mathcal{A}$.

Consideremos agora $x_{0} \in X \backslash \mathcal{A}$ um ponto qualquer, então $h\left(x_{0}\right)>0$. Consideremos também $\mathcal{O}_{\mu}(\mathcal{A})$ para algum $0<\mu<h\left(x_{0}\right)$ e, pela continuidade da função distância ao conjunto $\mathcal{A}$, seja $V$ uma vizinhança limitada de $x_{0}$ tal que $d(x, \mathcal{A})>\mu$ sempre que $x \in V$. Finalmente, seja $\tau>0$ de modo que $\gamma_{\tau}^{+}(V) \subset \mathcal{O}_{\mu}(\mathcal{A})$.

Daí conclui-se facilmente a continuidade de $h$ em $x_{0}$, uma vez que para $x \in V$ temos $h(x)=\sup _{0 \leq t \leq \tau} d(T(t) x, \mathcal{A})$, e como a família de aplicações $\{T(t): 0 \leq t \leq \tau\}$ é equicontínua em todos os pontos de $V$ e a função distância ao conjunto $\mathcal{A}$ é uniformemente contínua, a função $\left.h\right|_{V}: V \rightarrow \mathbb{R}$ resulta contínua, completando a prova da continuidade de $h$. 
Para ver que $h$ é não crescente ao longo de soluções, dados $x \in X$ e $t_{1}>0$ tem-se

$$
\begin{gathered}
h\left(T\left(t_{1}\right) x\right)=\sup _{t \geq 0} d\left(T(t) T\left(t_{1}\right) x, \mathcal{A}\right)=\sup _{t \geq 0} d\left(T\left(t+t_{1}\right) x, \mathcal{A}\right)= \\
\sup _{t \geq t_{1}} d(T(t) x, \mathcal{A}) \leq \sup _{t \geq 0} d(T(t) x, \mathcal{A})=h(x),
\end{gathered}
$$

como queríamos demonstrar.

Com a ajuda deste último resultado e das ferramentas desenvolvidas na seção anterior, podemos provar o resultado fundamental deste capítulo, o qual está contido na seguinte proposição.

Proposição 4.2.2. Seja $\left(A, A^{*}\right)$ um par atrator-repulsor para um semigrupo $\{T(t): t \geq 0\}$ em um espaço métrico $X$ possuindo atrator global $\mathcal{A}$.

Existe uma função $f: X \rightarrow \mathbb{R}$ satisfazendo as quatro propriedades seguintes:

(i) $f: X \rightarrow \mathbb{R}$ é uma função contínua (em todo o espaço $X$ ).

(ii) $f: X \rightarrow \mathbb{R}$ é não crescente ao longo de soluções de $T(\cdot)$.

(iii) $f^{-1}(0)=A$ e $f^{-1}(1) \cap \mathcal{A}=A^{*}$.

(iv) Dado $x \in X$, tem-se $f(T(t) x)=f(x)$ para todo $t \geq 0$ se, e somente se, $x \in$ $\left(A \cup A^{*}\right)$.

Um função $f: X \rightarrow \mathbb{R}$ com as propriedades listadas acima denomina-se função de Lyapunov do par atrator-repulsor $\left(A, A^{*}\right)$.

Demonstração: Em primeiro lugar, observemos que sendo $A$ e $A^{*}$ fechados disjuntos relativos de $\mathcal{A}$ e sendo $\mathcal{A}$ um fechado de $X$, deduz-se que $A$ e $A^{*}$ são fechados disjuntos de $X$, donde a função de Uryshon canônica associada ao par de fechados disjuntos $\left(A, A^{*}\right), l: X \rightarrow[0,1]$, dada por

$$
l(x):=\frac{d(x, A)}{d(x, A)+d\left(x, A^{*}\right)}, x \in X
$$

está bem definida, é uniformemente contínua em $X$ (pois, pondo $d_{0}:=d\left(A, A^{*}\right)>$ 0 , é fácil comprovar que $|l(x)-l(y)| \leq \frac{2}{d_{0}} d(x, y)$, quaisquer que sejam $x$ e $y$ em $\left.X\right)$ e, além disso verifica-se $l^{-1}(0)=A$ e $l^{-1}(1)=A^{*}$.

Definindo agora a função $k: X \rightarrow \mathbb{R}$ por

$$
k(x):=\sup _{t \geq 0} l(T(t) x)
$$


provemos que $k: X \rightarrow \mathbb{R}$ é contínua, não crescente ao longo de soluções de $\{T(t): t \geq 0\}$, com imagem contida no intervalo $[0,1]$ e tal que $k^{-1}(0)=A$ e $k^{-1}(1) \cap \mathcal{A}=A^{*}$.

Com efeito, que a imagem de $k: X \rightarrow \mathbb{R}$ está contida no intervalo [0,1] segue do fato de que $l(T(t) x) \in[0,1]$ para todo $x \in X$ e todo $t \geq 0$.

Por outro lado, dados $x \in X$ e $0 \leq t_{1} \leq t_{2}$ vemos que

$$
\begin{gathered}
k\left(T\left(t_{1}\right) x\right)=\sup _{t \geq 0} l\left(T(t) T\left(t_{1}\right) x\right)=\sup _{t \geq 0} l\left(T\left(t+t_{1}\right) x\right)=\sup _{t \geq t_{1}} l(T(t) x) \\
\geq \sup _{t \geq t_{2}} l(T(t) x)=\sup _{t \geq 0} l\left(T\left(t+t_{2}\right) x\right)=k\left(T\left(t_{2}\right) x\right)
\end{gathered}
$$

provando que é não crescente ao longo de soluções.

Claramente, $k(A)=\{0\}$ e $k\left(A^{*}\right)=\{1\}$, simplesmente pelo fato de que $A$ e $A^{*}$ são invariantes e a pela definição de $l$.

Agora, seja $x \in X$ tal que $k(x)=0$, então $l(T(t) x)=0$ para todo $t \geq 0$. Em particular, $0=l(T(0) x)=l(x)$ e, portanto, $x \in A$, ou seja $k^{-1}(0) \subset A$, provando a igualdade $k^{-1}(0)=A$. Por outro lado, seja $x \in \mathcal{A}$ tal que $k(x)=1$, se $x \notin A^{*}$, então $\omega(x) \subset A$. Pela continuidade de $l$ e do fato de que $\omega(x)$ atrai $x$ pelo semigrupo, conclui-se que $\lim _{t \rightarrow \infty} l(T(t) x)=0$. Logo existe $t_{0}>0$ tal que $1=k(x)=\sup _{0 \leq t \leq t_{0}} l(T(t) x)$, portanto, existe $t^{\prime} \in\left[0, t_{0}\right] \operatorname{com} l\left(T\left(t^{\prime}\right) x\right)=1$, isto é, $T\left(t^{\prime}\right) x \in \bar{A}^{*}$, e por isso, $\omega(x)=\omega\left(T\left(t^{\prime}\right) x\right) \subset A^{*}$, contradizendo $\omega(x) \subset A$ e assim, se $k(x)=1$ para algum $x \in \mathcal{A}$, obrigatoriamente $x \in A^{*}$, resultando destes cálculos que $k^{-1}(1) \cap \mathcal{A} \subset A^{*}$ e, consequentemente, $k^{-1}(1) \cap \mathcal{A}=A^{*}$.

Provemos agora que, se $x \in \mathcal{A}$ e $k(T(t) x)=k(x)$ para todo $t \geq 0$ então $x \in$ $\left(A \cup A^{*}\right)$. Suponhamos, caso contrário, que isso não se verifica, ou seja, que existe $x \in \mathcal{A} \backslash\left(A \cup A^{*}\right) \operatorname{com} k(T(t) x)=k(x)$ para todo $t \geq 0$. Então, pelo item $(i)$ do Lema 4.1.7, $\omega(x) \subset A$. Da difinição de $k$ e do fato de que $\omega(x)$ atrai $x$ pelo semigrupo temos $k(x)=\lim _{t \rightarrow \infty} k(T(t) x)=0, \operatorname{logo} x$ deve pertencer a $A$, pois como vimos, $k^{-1}(0)=A$, o que está em contradição com o fato de que $x \notin\left(A \cup A^{*}\right)$.

Agora, provemos a continuidade da função $k: X \rightarrow \mathbb{R}$, dividindo a demonstração nos três seguintes casos:

Caso 1) Continuidade de $k: X \rightarrow \mathbb{R}$ nos pontos de $A^{*}$.

Como para todo $x \in X$ vale $l(x) \leq k(x) \leq 1$, dados $x_{0} \in A^{*}$ e $x \in X$, quaisquer, temos

$$
\left|k(x)-k\left(x_{0}\right)\right|=1-k(x) \leq 1-l(x),
$$

donde se vê que a continuidade de $k: X \rightarrow \mathbb{R}$ em $x_{0}$ segue diretamente da continuidade de $l: X \rightarrow \mathbb{R}$ neste ponto.

Caso 2) Continuidade de $k: X \rightarrow \mathbb{R}$ nos pontos de $A$. 
Neste caso, dado $\varepsilon>0$, pela continuidade da função $l: X \rightarrow \mathbb{R}$ no conjunto compacto $A$, seja $\delta>0$ de maneira que $l\left(\mathcal{O}_{\delta}(A)\right) \subset[0, \varepsilon)$. Pelo Lema 4.1.2, ao $\delta>0$ acima corresponde $\delta^{\prime} \in(0, \delta)$ tal que $\gamma^{+}\left(\mathcal{O}_{\delta^{\prime}}(A)\right) \subset \mathcal{O}_{\delta}(A)$, donde conclui-se que $k\left(\mathcal{O}_{\delta^{\prime}}(A)\right) \subset[0, \varepsilon]$, pois dado $x \in \mathcal{O}_{\delta^{\prime}}(A)$ tem-se que $T(t) x \in \mathcal{O}_{\delta}(A)$ para todo $t \geq 0$, logo $l(T(t) x) \in[0, \varepsilon)$ para todo $t \geq 0$ e assim $k(x)=\sup _{t \geq 0} l(T(t) x) \in[0, \varepsilon]$, o que estabelece a continuidade de $k$ nos pontos de $A$.

Caso 3) Continuidade de $k: X \rightarrow \mathbb{R}$ nos pontos de $X \backslash\left(A \cup A^{*}\right)$.

Dado $x_{0} \in X \backslash\left(A \cup A^{*}\right)$, usando o item (ii) do Lema 4.1.7 tem-se que,

$$
\text { ou } \lim _{t \rightarrow \infty} d\left(T(t) x_{0}, A\right)=0 \text { ou } \lim _{t \rightarrow \infty} d\left(T(t) x_{0}, A^{*}\right)=0 .
$$

Suponhamos primeiramente que se tenha $\lim _{t \rightarrow \infty} d\left(T(t) x_{0}, A^{*}\right)=0$. Então, em particular, $k\left(x_{0}\right)=1$. Agora, dado $\varepsilon>0$ escolhamos, pela continuidade da função $l: X \rightarrow \mathbb{R}$ no conjunto compacto $A^{*}$, uma vizinhança $V$ de $A^{*}$ em $X$ tal que $l(V) \subset(1-\varepsilon, 1]$. Seja também, $t_{0}>0$ de maneira que $T\left(t_{0}\right) x_{0} \in V$. Agora, pela continuidade da aplicação $T\left(t_{0}\right): X \rightarrow X$, seja $U$ uma vizinhança de $x_{0}$ tal que $T\left(t_{0}\right) U \subset V$, donde segue-se que para todo $x \in U$ tem-se $k(x)>1-\varepsilon$ (pois $T\left(t_{0}\right) x \in V$ e então $\left.1-\varepsilon<l\left(T\left(t_{0}\right) x\right) \leq k(x)\right)$, o que nos mostra a continuidade de $k$ em $x_{0}$ quando ocorrer $\lim _{t \rightarrow \infty} d\left(T(t) x_{0}, A^{*}\right)=0$.

Caso seja $\lim _{t \rightarrow \infty} d\left(T(t) x_{0}, A\right)=0$, como $x_{0} \in X \backslash\left(A \cup A^{*}\right)$, vem que $l\left(x_{0}\right)>0$. Então, seja $\delta>0$ tal que $l\left(\mathcal{O}_{\delta}(A)\right) \subset\left[0, \frac{l\left(x_{0}\right)}{2}\right)$ e, novamente pelo Lema 4.1.2, seja $\delta^{\prime} \in(0, \delta)$ de modo que $\gamma^{+}\left(\mathcal{O}_{\delta^{\prime}}(A)\right) \subset \mathcal{O}_{\delta}(A)$. Sejam também $t_{0}$ tal que $T(t) x_{0} \in \mathcal{O}_{\delta^{\prime}}(A)$ para todo $t \geq t_{0}$ e mais uma vez, pela continuidade da aplicação $T\left(t_{0}\right): X \rightarrow X$, seja $U_{1}$ uma vizinhança de $x_{0}$ em $X$ com $T\left(t_{0}\right) U_{1} \subset \mathcal{O}_{\delta^{\prime}}(A)$, donde segue-se que para todo $x \in U_{1}$ temos $T\left(t_{0}\right) x \in \mathcal{O}_{\delta^{\prime}}(A)$ e por isso tem-se $T(t) x \in \mathcal{O}_{\delta}(A)$ para todo $t \geq t_{0}$. Finalmente, seja, também pela continuidade de $l, U_{2}$ uma vizinhança de $x_{0}$ em $X$ tal que $l(x)>\frac{l\left(x_{0}\right)}{2}$ para todo $x \in U_{2}$ e ponhamos $U:=U_{1} \cap U_{2}$, logo para todo $x \in U$ temos $k(x)=\sup _{0 \leq t \leq t_{0}} l(T(t) x)$, pois para $x \in U, k(x) \geq l(x)>\frac{l\left(x_{0}\right)}{2}$ e $T(t) x \in \mathcal{O}_{\delta}(A)$ para todo $t \geq t_{0}$. Consequentemente $l(T(t) x)<\frac{l\left(x_{0}\right)}{2}<k(x)$ para todo $t \geq t_{0}$. E usando argumentos já utilizados anteriormente conclui-se que $\left.k\right|_{U}: U \rightarrow \mathbb{R}$ é contínua em $x_{0}$, o que termina a prova do terceiro caso e, portanto, conclui-se a prova da continuidade da função $k: X \rightarrow \mathbb{R}$.

Finalmente, seja $h: X \rightarrow \mathbb{R}$ a função definida por $h(x):=\sup _{t \geq 0} d(T(t) x, \mathcal{A}), x \in X$, dada no Lema 4.2.1. Nossa função $f: X \rightarrow \mathbb{R}$ buscada será dada por

$$
f(x):=k(x)+h(x), x \in X .
$$

A continuidade de $f: X \rightarrow \mathbb{R}$ segue simplesmente da continuidade de $k$, estudada acima, e da continuidade de $h$, dada no Lema 4.2.1, estabelecendo a propriedade $(i)$. 
O fato de que $f$ é não crescente ao longo de soluções segue do fato de que a mesma propriedade verifica-se tanto para $k$ quanto para $h$, provando $(i i)$.

Agora, claramente, $f(A)=\{0\}$ e se, reciprocamente, $f(x)=0$ para algum $x \in$ $X$ tem-se, em particular, que tanto $h(x)$ quanto $k(x)$ são nulos, por isso, $x \in \mathcal{A}$ e, pela correspondente propriedade para $k$ estudada anteriormente, vem que $x \in A$, provando que $f^{-1}(0)=A$. Por outro lado, como $\left.f\right|_{\mathcal{A}}=\left.k\right|_{\mathcal{A}}$ temos, pela correspondente propriedade de $k$, que $f$ é tal que $f^{-1}(1) \cap \mathcal{A}=k^{-1}(1) \cap \mathcal{A}=A^{*}$, terminando a prova do item $($ iii) .

Agora, a propriedade (iv) pode ser provada assim:

Seja $x \in X$ tal que $f(T(t) x)=f(x)$ para todo $t \geq 0$. Se $x \in \mathcal{A}$, esta última igualdade é a mesma que $k(T(t) x)=k(x)$. Como vimos acima, isto implica $x \in\left(A \cup A^{*}\right)$ e então não há o que provar. Suponhamos então que $x \in X \backslash \mathcal{A}$. Afirmamos que $\lim _{t \rightarrow \infty} d\left(T(t) x, A^{*}\right)=0$, porque se fosse $\lim _{t \rightarrow \infty} d(T(t) x, A)=0$, teríamos, em particular, que

$$
f(x)=\lim _{t \rightarrow \infty} f(T(t) x)=\lim _{t \rightarrow \infty} k(T(t) x)+\lim _{t \rightarrow \infty} h(T(t) x)=0+0=0
$$

obrigando $x$ a estar em $A \subset \mathcal{A}$, contrariando que $x \in X \backslash \mathcal{A}$, e assim é certo que

$$
\lim _{t \rightarrow \infty} d\left(T(t) x, A^{*}\right)=0
$$

Nestas condições, afirmamos também que $f(x)=1$, pois aplicando o mesmo raciocínio que nos deu (4.2.4), concluímos que

$$
f(x)=\lim _{t \rightarrow \infty} f(T(t) x)=\lim _{t \rightarrow \infty} k(T(t) x)+\lim _{t \rightarrow \infty} h(T(t) x)=1+0=1 .
$$

Mas, este fato é uma contradição, uma vez que, sendo $k(x) \geq k(T(t) x)$ para todo $t \geq$ 0, vem que $1=\lim _{t \rightarrow \infty} k(T(t) x) \leq k(x) \leq 1$, consequentemente $k(x)=1$. Entretanto, $f(x)=k(x)+h \stackrel{t \rightarrow \infty}{(x)}$, forçando $h(x)$ ser igual a zero, o que é um absurdo, pois estando $x$ fora de $\mathcal{A}$ devemos ter $0<d(x, \mathcal{A}) \leq h(x)$ e esta contradição nos faz concluir que, se $f(T(t) x)=f(x)$ para todo $t \geq 0$, então $x \in \mathcal{A}$ e portanto $x \in\left(A \cup A^{*}\right)$, completando a demonstração do item $(i v)$ e também da proposição.

Observemos que o papel da função $h: X \rightarrow \mathbb{R}$ (introduzida no Lema 4.2.1) na prova da última proposição é reconhecer os pontos de $X$ sobre os quais $f: X \rightarrow \mathbb{R}$ é constante sobre suas semiórbitas positivas, como sendo apenas os pontos de $\mathcal{A}$. Propriedade que a função $k: X \rightarrow \mathbb{R}$, sozinha, pode não possuir ${ }^{1}$.

\footnotetext{
${ }^{1}$ Com efeito, se existir $x \in X \backslash \mathcal{A}$ tal que $\lim _{t \rightarrow \infty} d\left(T(t) x, A^{*}\right)=0$, então $k(T(t) x)=k(x)$ para todo $t \geq 0, \operatorname{com} x \notin A \cup A^{*}$.
} 
Devemos destacar também que, a hipótese de que cada $T(t): X \rightarrow X$ é um homeomorfismo é fortemente usada na prova da continuidade da função de Lyapunov de um par atrator-repulsor que se encontra em [12]. Por exemplo, por meio desta hipótese, lá obtém-se que, dada uma vizinhança aberta $U$ de um atrator local $A$, tem-se imediatamente que $T(t) U$ é também uma vizinhança aberta de $A$ para todo $t$. Como neste trabalho não assumimos esta hipótese de grupo, usamos o Lema 4.1.2 para substituir esta propriedade como, por exemplo, na prova do Caso 2 acima.

Finalmente, notemos que a prova do Caso 3 da proposição anterior não aparece em [12], uma vez que lá o autor não considera a continuidade em pontos que estão fora do atrator $\mathcal{A}$, pois sua função de Lyapunov é definida apenas em conjuntos compactos invariantes e não em espaços de fases arbitrários como fazemos aqui. Nestas condições, para incluirmos a continuidade em pontos fora do atrator foi necessário estabelecer a conclusão (ii) do Lema 4.1.7.

\subsection{Decomposição de Morse}

Agora, apresentamos a noção de decomposição de Morse para um atrator de um semigrupo em um espaço métrico. Este conceito funciona como uma ponte que nos permite passar de um semigrupo de tipo gradiente para um semigrupo gradiente.

Comecemos observando que, se $A_{1}$ e $A_{2}$ são atratores locais para $T(\cdot) \operatorname{com} A_{1} \subset A_{2}$, então, simplesmente aplicando a definição de repulsor complementar, conclui-se que $A_{2}^{*} \subset A_{1}^{*}$.

Definição 4.3.1. Seja $\{T(t): t \geq 0\}$ um semigrupo em um espaço métrico $X$ possuindo atrator global $\mathcal{A}$.

Dada uma cadeia crescente formada por $n+1$ atratores locais da forma

$$
\varnothing=: A_{0} \subset A_{1} \subset \cdots \subset A_{n-1} \subset A_{n}:=\mathcal{A}
$$

e considerando a cadeia decrescente de seus repulsores complementares

$$
\varnothing=A_{n}^{*} \subset A_{n-1}^{*} \subset \cdots \subset A_{1}^{*} \subset A_{0}^{*}=\mathcal{A},
$$

pondo, para cada $j=1,2, \cdots, n, M_{j}:=A_{j} \cap A_{j-1}^{*}$, a n-upla ordenada

$$
\mathbf{D}:=\left(M_{1}, M_{2}, \cdots, M_{n}\right)
$$

chama-se uma decomposição de Morse do atrator $\mathcal{A}$ e os conjuntos $M_{j}$ 's chamamse os conjuntos de Morse desta decomposição. 
As considerações que seguem nos mostram como construir uma decomposição de Morse para o atrator de um semigrupo de tipo gradiente. Para isso, o lema a seguir desempenha um papel fundamental.

Lema 4.3.2. Sejam $\{T(t): t \geq 0\}$ um semigrupo em um espaço métrico $X$ de tipo gradiente com respeito à família disjunta $\Xi=\left\{\Xi_{1}, \Xi_{2}, \cdots, \Xi_{n}\right\}$ de conjuntos invariantes isolados limitados e $\mathcal{A}$ seu atrator global. Então, algum dos conjuntos invariantes isolados pertencentes a $\boldsymbol{\Xi}$ é um atrator local para $T(\cdot)$.

Demonstração: Com efeito, em primeiro lugar, afirmamos que existe $j=1,2, \cdots, n$ tal que para todo $\delta \in\left(0, \delta_{0}\right)$ existe $\delta^{\prime} \in(0, \delta)$ de maneira que

$$
\gamma^{+}\left(\mathcal{O}_{\delta^{\prime}}\left(\Xi_{j}\right)\right) \subset \mathcal{O}_{\delta}\left(\Xi_{j}\right)
$$

onde $\delta_{0}$ é tal que $\mathcal{O}_{\delta_{0}}\left(\Xi_{i}\right) \cap \mathcal{O}_{\delta_{0}}\left(\Xi_{r}\right)=\varnothing$ sempre que $i \neq r$ e $\Xi_{i}$ é o invariante maximal contido em $\mathcal{O}_{\delta_{0}}\left(\Xi_{i}\right)$ para $i=1,2, \cdots, n$.

Caso contrário, existiriam $\delta>0$ e para cada $j=1,2, \cdots, n$ uma sequência $\left(t_{k}^{(j)}\right)_{k \in \mathbb{N}}$ de números positivos e $\left(x_{k}^{(j)}\right)_{k \in \mathbb{N}}$ de pontos de $X$ tais que para todo $j$ e todo $k$

$$
\begin{gathered}
d\left(x_{k}^{(j)}, \Xi_{j}\right)<\frac{1}{k}, \\
d\left(T\left(t_{k}^{(j)}\right) x_{k}^{(j)}, \Xi_{j}\right)=\delta .
\end{gathered}
$$

$\mathrm{e}$

$$
d\left(T(t) x_{k}^{(j)}, \Xi_{j}\right)<\delta \text { sempre que } t \in\left[0, t_{k}^{(j)}\right)
$$

Então, pondo, para cada $j=1,2, \cdots, n$ e cada natural $k, \xi_{k}^{(j)}(t):=T\left(t+t_{k}^{(j)}\right) x_{k}^{(j)}$ para $t \in\left[-t_{k}^{(j)}, \infty\right)$, como $t_{k}^{(j)} \underset{k \rightarrow \infty}{\rightarrow} \infty$, pelo Lema 3.2.3, podemos supor que para cada $j=1,2, \cdots, n$ existe uma solução global $\xi_{j}: \mathbb{R} \rightarrow X$ para $T(\cdot)$ tal que $\xi_{k}^{(j)} \underset{k \rightarrow \infty}{\rightarrow} \xi_{j}$ uniformemente sobre compactos da reta e do fato de que $(T(\cdot), \boldsymbol{\Xi})$ é de tipo gradiente seguiria que para cada $j=1,2, \cdots, n$

$$
\lim _{t \rightarrow-\infty} d\left(\xi_{j}(t), \Xi_{j}\right)=0
$$

Logo, novamente pelo fato de que $(T(\cdot), \Xi)$ é de tipo gradiente, cada solução $\xi_{j}$ deveria convergir, quando $t \rightarrow \infty$, para algum invariante isolado em $\boldsymbol{\Xi}$, o que produziria uma estrutura homoclínica em $\mathcal{A}$ contradizendo que $(T(\cdot), \boldsymbol{\Xi})$ é de tipo gradiente, completando a prova de nossa afirmação.

Agora, levando em conta que nossa afirmação é correta, seja $\Xi_{j} \in \boldsymbol{\Xi}$ para o qual ela é válida. Então, dado $\delta \in\left(0, \delta_{0}\right)$ escolhamos $\delta^{\prime} \in(0, \delta)$ de maneira que

$$
\gamma^{+}\left(\mathcal{O}_{\delta^{\prime}}\left(\Xi_{j}\right)\right) \subset \mathcal{O}_{\delta}\left(\Xi_{j}\right)
$$


donde segue-se trivialmente que

$$
\omega\left(\mathcal{O}_{\delta^{\prime}}\left(\Xi_{j}\right)\right) \subset \overline{\mathcal{O}_{\delta}\left(\Xi_{j}\right)} \subset \mathcal{O}_{\delta_{0}}\left(\Xi_{j}\right)
$$

$\operatorname{logo}$, como $\Xi_{j}$ é o invariante maximal contido em $\mathcal{O}_{\delta_{0}}\left(\Xi_{j}\right)$ e sendo $\omega\left(\mathcal{O}_{\delta^{\prime}}\left(\Xi_{j}\right)\right)$ um conjunto invariante, resulta

$$
\omega\left(\mathcal{O}_{\delta^{\prime}}\left(\Xi_{j}\right)\right) \subset \Xi_{j}
$$

e como $\Xi_{j} \subset \omega\left(\mathcal{O}_{\delta^{\prime}}\left(\Xi_{j}\right)\right)$, obtém-se a igualdade $\omega\left(\mathcal{O}_{\delta^{\prime}}\left(\Xi_{j}\right)\right)=\Xi_{j}$, mostrando que $\Xi_{j}$ é um atrator local, como queríamos.

Seja $\{T(t): t \geq 0\}$ um semigrupo de tipo gradiente generalizado com respeito à família disjunta $\Xi=\left\{\Xi_{1}, \Xi_{2} \cdots, \Xi_{n}\right\}$ de conjuntos invariantes isolados e $\mathcal{A}$ seu atrator global. Pelo lema anterior, seja $\Xi_{\sigma(1)} \in \boldsymbol{\Xi}$ um atrator local para $T(\cdot)$ e $\Xi_{\sigma(1)}^{*}:=\{x \in$ $\left.\mathcal{A}: \omega(x) \cap \Xi_{\sigma(1)}=\varnothing\right\}$ seu repulsor complementar, então, como os elementos de $\boldsymbol{\Xi}$ são fechados invariantes e disjuntos entre si, segue-se que para todo $j \neq \sigma(1), \Xi_{j} \subset \Xi_{\sigma(1)}^{*}$.

Considerando $\left\{T_{1}(t): t \geq 0\right\}$ a restrição de $\{T(t): t \geq 0\}$ ao conjunto invariante $\Xi_{\sigma(1)}^{*}$, resulta imediatamente que $\left\{T_{1}(t): t \geq 0\right\}$ herda de $\{T(t): t \geq 0\}$ as propriedades que definem os semigrupos de tipo gradiente, ou seja, que $\left\{T_{1}(t): t \geq 0\right\}$ é de tipo gradiente no espaço métrico $\Xi_{\sigma(1)}^{*}$ com respeito à família finita disjunta $\left\{\Xi_{j}: j \neq \sigma(1)\right\}$ de invariantes isolados. Então, novamente por aplicação do lema anterior, seja $\Xi_{\sigma(2)} \in\left\{\Xi_{j}: j \neq \sigma(1)\right\}$ um atrator local para $\left\{T_{1}(t): t \geq 0\right\}$ em $\Xi_{\sigma(1)}^{*}$. Seja, por simplicidade de notação, $\Xi_{\sigma(2)}^{*}:=\left\{x \in \Xi_{\sigma(1)}^{*}: \omega(x) \cap \Xi_{\sigma(2)}=\varnothing\right\}$ o repulsor complementar de $\Xi_{\sigma(2)}$ em $\Xi_{\sigma(1)}^{*}$. Como antes, temos que $\Xi_{j} \subset \Xi_{\sigma(2)}^{*}$ sempre que $j \notin\{\sigma(1), \sigma(2)\}$ e existe $\Xi_{\sigma(3)} \in\left\{\Xi_{j}: j \notin\{\sigma(1), \sigma(2)\}\right\}$ um atrator local para a restrição, $\left\{T_{2}(t): t \geq 0\right\}$, de $\{T(t): t \geq 0\}$ ao conjunto invariante $\Xi_{\sigma(2)}^{*}$, o qual é de tipo gradiente em $\Xi_{\sigma(2)}^{*}$ com respeito à família $\left\{\Xi_{j}: j \notin\{\sigma(1), \sigma(2)\}\right\}$. Pondo $\Xi_{\sigma(3)}^{*}:=\left\{x \in \Xi_{\sigma(2)}^{*}: \omega(x) \cap \Xi_{\sigma(3)}=\varnothing\right\}$ para indicar o repulsor de $\Xi_{\sigma(3)}$ em $\Xi_{\sigma(2)}^{*}$, podemos prosseguir com o raciocínio até que se esgotem todos os elementos de $\boldsymbol{\Xi}$, donde, ao final, obtemos que $\Xi_{\sigma(1)}$ é um atrator local para $\{T(t): t \geq 0\}$ em $X$ e para cada $i \geq 2, \Xi_{\sigma(i)}$ é um atrator local para a restrição de $\{T(t): t \geq 0\}$ ao repulsor $\Xi_{\sigma(i-1)}^{*}$ (no sentido da construção acima).

Evidentemente, pondo $I_{n}:=\{1,2, \cdots, n\}$, a aplicação $\sigma: I_{n} \rightarrow I_{n}$, definida por meio do procedimento acima (portanto dependendo tanto do semigrupo $T(\cdot)$ quanto da família de conjuntos invariantes isolados $\boldsymbol{\Xi})$, é uma permutação do conjunto $I_{n}$, ou seja, uma bijeção do conjunto $I_{n}$ em si mesmo.

Sejam $\sigma: I_{n} \rightarrow I_{n}$ uma permutação como definida acima e $\mathbf{D}:=\left(\Xi_{\sigma(1)}, \Xi_{\sigma(2)}, \cdots, \Xi_{\sigma(n)}\right)$ a $n$-upla ordenada obtida reordenando-se os elementos do conjunto $\boldsymbol{\Xi}$ segundo a bijeção 
$\sigma$. Então, para toda solução global limitada $\xi: \mathbb{R} \rightarrow X$ com

$$
\lim _{t \rightarrow-\infty} d\left(\xi(t), \Xi_{\sigma(i)}\right)=0 \text { e } \lim _{t \rightarrow \infty} d\left(\xi(t), \Xi_{\sigma(j)}\right)=0,
$$

tem-se $i \geq j$.

Com efeito, observemos primeiramente que se $A$ é um atrator local para $T(\cdot)$ e $A^{*}$ seu repulsor complementar, então para toda solução global $\zeta: \mathbb{R} \rightarrow X \operatorname{com} \zeta(0) \in \Xi^{*}$, tem-se $\zeta(t) \in \Xi^{*}$ qualquer que seja $t$ real, simplesmente porque para todo real $t$ vale $\omega(\zeta(t))=\omega(\zeta(0))$.

Agora, notemos que se $j=1$ não há nada o que demonstrar.

Se $j=2$, necessariamente $\xi(0) \in \Xi_{\sigma(1)}^{*}$, porque, como $\xi(0) \in \mathcal{A}$, se $\xi(0) \notin \Xi_{\sigma(1)}^{*}$ então $\omega(\xi(0)) \cap \Xi_{\sigma(1)} \neq \varnothing$ e portanto $\omega(\xi(0)) \subset \Xi_{\sigma(1)}$, o que contradiz $\sigma(1) \neq \sigma(2)$ (ou seja, contradiz o fato de que $\sigma$ é uma bijeção). Desta forma, $\xi(0) \in \Xi_{\sigma(1)}^{*}$ e portanto, como observamos acima, $\xi(t) \in \Xi_{\sigma(1)}^{*}$ para todo $t \in \mathbb{R}$. Pela definição da permutação $\sigma, \Xi_{\sigma(k)} \subset \Xi_{\sigma(1)}^{*}$ para todo $k \geq 2, \log 0 i \geq 2=j$.

Para provar o caso geral, primeiro provemos que, se $j \geq 3,3 \leq k \leq j$, (4.3.5) se verifica e $\xi(0) \in \Xi_{\sigma(k-2)}^{*}$, então $\xi(0) \in \Xi_{\sigma(k-1)}^{*}$.

Observemos primeiramente que, se $k=3$ e $\xi(0) \in \Xi_{\sigma(1)}^{*} \operatorname{mas} \xi(0) \notin \Xi_{\sigma(2)}^{*}$ então, como $\xi(t) \in \Xi_{\sigma(1)}^{*}$ para todo real $t$ e $\Xi_{\sigma(2)}^{*}$ é o repulsor de $\Xi_{\sigma(2)}\left(\right.$ em $\left.\Xi_{\sigma(1)}^{*}\right)$ segue-se que $\omega(\xi(0)) \subset \Xi_{\sigma(2)}$ o que contradiz $j \geq 3$, já que $\omega(\xi(0)) \subset \Xi_{\sigma(j)}$ devido a (4.3.5).

Se $k>3, \xi(0) \in \Xi_{\sigma(k-2)}^{*} \operatorname{mas} \xi(0) \notin \Xi_{\sigma(k-1)}^{*}$, como $\xi(t) \in \Xi_{\sigma(k-2)}^{*}$ para todo $t$ real e $\Xi_{\sigma(k-1)}^{*}$ é o repulsor de $\Xi_{\sigma(k-1)}\left(\mathrm{em} \Xi_{\sigma(k-2)}^{*}\right)$ segue-se que $\omega(\xi(0)) \subset \Xi_{\sigma(k-1)}$, obrigando $\Xi_{\sigma(k-1)}=\Xi_{\sigma(j)}$, ou seja, $k-1=j$ contradizendo $k \leq j$.

Destes fatos resulta que se (4.3.5) se verifica, $3 \leq k \leq j$ e $\xi(0) \in \Xi_{\sigma(k-2)}^{*}$, então $\xi(0) \in \Xi_{\sigma(k-1)}^{*}$. Assim, em particular, vemos que $\xi(0) \in \Xi_{\sigma(j-1)}^{*}$ e portanto $\xi(t) \in \Xi_{\sigma(j-1)}^{*}$ qualquer que seja $t \in \mathbb{R}$ e como $\Xi_{\sigma(l)} \subset \Xi_{\sigma(j-1)}^{*}$ sempre que $l \geq j$, necessariamente $i \geq j$, estabelecendo o caso geral e a prova de nossa afimação.

A seguir provamos que a $n$-upla ordenada $\mathbf{D}=\left(\Xi_{\sigma(1)}, \Xi_{\sigma(2)}, \cdots, \Xi_{\sigma(n)}\right)$, obtida reordenando-se os elementos do conjunto $\boldsymbol{\Xi}$ por meio da permutação $\sigma$, como explicamos acima, determina uma decomposição de Morse para o atrator global $\mathcal{A}$ do semigrupo $T(\cdot)$ de tipo gradiente com respeito a $\boldsymbol{\Xi}$. Primeiro fixemos algumas notações.

Definamos:

$$
\begin{aligned}
A_{0}:=\varnothing, A_{1}:=\Xi_{\sigma(1)} \text { e para } j & =2,3, \cdots, n \\
A_{j} & :=A_{j-1} \cup W^{u}\left(\Xi_{\sigma(j)}\right) .
\end{aligned}
$$

Observemoe que, com estas definições, para $j=1,2, \cdots, n$

$$
A_{j}=\bigcup_{i=1}^{j} W^{u}\left(\Xi_{\sigma(i)}\right) \text {, }
$$


donde, em particular, obtém-se

$$
\varnothing=A_{0} \subset A_{1} \subset \cdots \subset A_{n}=\bigcup_{i=1}^{n} W^{u}\left(\Xi_{\sigma(i)}\right)=\mathcal{A} .
$$

O resultado a seguir pode ser provado usando-se o Teorema 1.8 em [20]. Contudo, resolvemos apresentar uma demonstração alternativa que explora as propriedades dos semigrupos de tipo gradiente.

Teorema 4.3.3. Sejam $\{T(t): t \geq 0\}$ um semigrupo em um espaço métrico $X$ de tipo gradiente com respeito à família finita disjunta $\Xi=\left\{\Xi_{1}, \Xi_{2}, \cdots, \Xi_{n}\right\}$ de conjuntos invariantes isolados limitados e $\mathcal{A}$ seu atrator global.

Sejam também, $\sigma: I_{n} \rightarrow I_{n}$ uma permutação obtida por meio do procedimento que explicamos acima e $\mathbf{D}=\left(\Xi_{\sigma(1)}, \Xi_{\sigma(2)}, \cdots, \Xi_{\sigma(n)}\right)$.

Então, para cada $j=0,1, \cdots, n$ o conjunto $A_{j}$ como definido em (4.3.6) é um atrator local para $T(\cdot)$ em $X$. Além disso, para todo $j=1,2, \cdots, n$ verifica-se que

$$
A_{j} \cap A_{j-1}^{*}=\Xi_{\sigma(j)}
$$

Ou seja, a reordenação $\mathbf{D}=\left(\Xi_{\sigma(1)}, \Xi_{\sigma(2)}, \cdots, \Xi_{\sigma(n)}\right)$ dos conjuntos invariantes isolados de $\boldsymbol{\Xi}$ é uma decomposição de Morse para o atrator global $\mathcal{A}$.

Demonstração: Em primeiro lugar, afirmamos que existe $\delta_{0}>0$ de maneira que para todo $j=1,2, \cdots, n-1$ vale

$$
A_{j} \cap \mathcal{O}_{\delta_{0}}\left(\bigcup_{i=j+1}^{n} \Xi_{\sigma(i)}\right)=\varnothing .
$$

Façamos a prova desta afirmação por indução em $j$.

O caso $j=1$ segue dos fatos de que $A_{1}=\Xi_{\sigma(1)}$ e que o conjunto $\Xi$ é uma família finita disjunta de invariantes isolados.

Suponhamos que a afirmação é certa para $j-1$ e a provemos para $j$. Caso nossa afirmação seja falsa para $j$, podemos encontrar uma sequência de pontos $\left(x_{k}\right)_{k \in \mathbb{N}}$ em $A_{j}$ tal que para todo natural $k$

$$
d\left(x_{k}, \bigcup_{i=j+1}^{n} \Xi_{\sigma(i)}\right)<\frac{1}{k}
$$

Do fato de que $\boldsymbol{\Xi}$ é finito podemos supor que, na verdade, para todo $k$ tem-se

$$
d\left(x_{k}, \Xi_{\sigma\left(i_{0}\right)}\right)<\frac{1}{k}
$$

para um certo $j+1 \leq i_{0} \leq n$ fixo. 
Como $A_{j}:=A_{j-1} \cup W^{u}\left(\Xi_{\sigma(j)}\right)$ e estamos supondo a afirmação válida para $j-1$, podemos também supor que $x_{k} \in W^{u}\left(\Xi_{\sigma(j)}\right)$ para todo natural $k$.

Assim, para cada $k \in \mathbb{N}$ existe uma solução global de $T(\cdot), \xi_{k}: \mathbb{R} \rightarrow X$ com $\xi_{k}(0)=x_{k}$, tal que

$$
\lim _{t \rightarrow-\infty} d\left(\xi_{k}(t), \Xi_{\sigma(j)}\right)=0 .
$$

Levando em conta (4.3.8) é possível, para cada natural $k$, encontrar um par de números reais $t_{k}^{\prime}$ e $t_{k}$, com $t_{k}^{\prime}<0 \leq t_{k}$, de maneira que

$$
d\left(\xi_{k}\left(t_{k}^{\prime}\right), \Xi_{\sigma\left(i_{0}\right)}\right)=\delta
$$

e

$$
d\left(\xi_{k}(t), \Xi_{\sigma\left(i_{0}\right)}\right)<\delta \text { sempre que } t \in\left(t_{k}^{\prime}, t_{k}\right]
$$

onde $\delta>0$ é tal que as $\delta$-vizinhanças dos elementos de $\boldsymbol{\Xi}$ são disjuntas entre si.

Então, definamos, para cada $k, \zeta_{k}: \mathbb{R} \rightarrow X$ pondo $\zeta_{k}(t):=\xi_{k}\left(t+t_{k}^{\prime}\right), t \in \mathbb{R}$.

Agora, observemos que, se (passando para uma subsequência se necessário) $t_{k}-$ $t_{k}^{\prime} \underset{k \rightarrow \infty}{\rightarrow} \infty$, então $d\left(\zeta_{k}(t), \Xi_{\sigma\left(i_{0}\right)}\right)<\delta$ para todo $t>0$ sempre que $k \in \mathbb{N}$ é suficientemente grande.

Por outro lado, se a sequência $\left(t_{k}-t_{k}^{\prime}\right)_{k \in \mathbb{N}}$ é limitada, podemos supor que existe $\tau_{0} \in \mathbb{R}$ de modo que $t_{k}-t_{k}^{\prime} \underset{k \rightarrow \infty}{\rightarrow} \tau_{0}$ e, por isso, a sequência $\left(t_{k}^{\prime}\right)_{k \in \mathbb{N}}$ deve também ser limitada pois, caso contrário, poderíamos supor $t_{k}^{\prime} \underset{k \rightarrow \infty}{\rightarrow}-\infty$ e portanto

$$
t_{k}^{\prime}=t_{k}-\left(t_{k}-t_{k}^{\prime}\right) \geq-\left(t_{k}-t_{k}^{\prime}\right) \underset{k \rightarrow \infty}{\rightarrow}-\tau_{0},
$$

contradizendo $\tau_{0} \in \mathbb{R}$. Assim, podemos assumir $t_{k \rightarrow \infty}^{\prime} \underset{k \rightarrow \infty}{\prime} t_{0}^{\prime}$

Agora, nestas condições, é fácil ver que a sequência $\left(\zeta_{k}\right)_{k \in \mathbb{N}}$ possui uma subsequência convergindo uniformemente sobre compactos da reta para uma solução global $\zeta^{(1)}: \mathbb{R} \rightarrow$ $X$ que não está contida em $\Xi_{\sigma\left(i_{0}\right)}$ e satisfaz

$$
\lim _{t \rightarrow \infty} d\left(\zeta^{(1)}(t), \Xi_{\sigma\left(i_{0}\right)}\right)=0^{2}
$$

\footnotetext{
${ }^{2}$ Isto pode ser visto assim:

(1) No caso onde $t_{k}-t_{k}^{\prime} \underset{k \rightarrow \infty}{\rightarrow} \infty$, temos que dado $t>0$ existe $k(t) \in \mathbb{N}$ tal que $0<t<t_{k}-t_{k}^{\prime}$ sempre que $k \geq k(t)$, isto é, $t_{k}^{\prime}<t+t_{k}^{\prime}<t_{k}$ sempre que $k \geq k(t)$ e por isso $d\left(\zeta_{k}(t), \Xi_{\sigma\left(i_{0}\right)}\right)<\delta$ sempre que $t>0$ e $k \geq k(t)$ donde, passando ao limite, obtém-se $d\left(\zeta^{(1)}(t), \Xi_{\sigma\left(i_{0}\right)}\right) \leq \delta$ toda vez que $t \geq 0$, e agora usando o fato de que o semigrupo é de tipo gradiente e a forma com a qual escolhemos $\delta>0$ concluímos que $\lim _{t \rightarrow \infty} d\left(\zeta^{(1)}(t), \Xi_{\sigma\left(i_{0}\right)}\right)=0$.

(2) No caso onde $t_{k}-t_{k}^{\prime} \underset{k \rightarrow \infty}{\rightarrow} \tau_{0}$, podemos supor $t_{k}^{\prime} \underset{k \rightarrow \infty}{\rightarrow} t_{0}^{\prime}$, e então, de $\zeta_{k}\left(-t_{k}^{\prime}\right)=\xi_{k}(0)=x_{k}$, graças a (4.3.8), resulta que $\zeta^{(1)}\left(-t_{0}^{\prime}\right) \in \Xi_{\sigma\left(i_{0}\right)}$, donde $\zeta^{(1)}(t) \in \Xi_{\sigma\left(i_{0}\right)}$ sempre que $t \geq-t_{0}^{\prime}$, e portanto, evidentemente, $\lim _{t \rightarrow \infty} d\left(\zeta^{(1)}(t), \Xi_{\sigma\left(i_{0}\right)}\right)=0$.

Os casos que seguem justificam-se analogamente.
} 
Sendo $(T(\cdot), \boldsymbol{\Xi})$ um semigrupo de tipo gradiente, obtém-se a existência de $\Xi_{\sigma\left(i_{1}\right)} \in$ $\Xi$, com $i_{1} \neq i_{0}$, de modo que

$$
\lim _{t \rightarrow-\infty} d\left(\zeta^{(1)}(t), \Xi_{\sigma\left(i_{1}\right)}\right)=0
$$

Pelo que demonstramos acima, obrigatoriamente $i_{1}>i_{0}$.

Por outro lado, para cada natural $r$ podemos encontrar um real $\tau_{r}$ de maneira que

$$
d\left(\zeta^{(1)}\left(\tau_{r}\right), \Xi_{\sigma\left(i_{1}\right)}\right)<\frac{1}{r} .
$$

Seja $\zeta_{r}^{(1)}: \mathbb{R} \rightarrow X$ dada por $\zeta_{r}^{(1)}(t):=\zeta^{(1)}\left(t+\tau_{r}\right), t \in \mathbb{R}$.

Levando em conta (4.3.10) concluímos que, para cada natural $r$, existe um par de números reais $s_{r}^{\prime}$ e $s_{r}$, com $s_{r}^{\prime}<0 \leq s_{r}$, de modo que

$$
d\left(\zeta_{r}^{(1)}\left(s_{r}^{\prime}\right), \Xi_{\sigma\left(i_{1}\right)}\right)=\delta
$$

e

$$
d\left(\zeta_{r}^{(1)}(t), \Xi_{\sigma\left(i_{1}\right)}\right)<\delta \text { sempre que } t \in\left(s_{r}^{\prime}, s_{r}\right],
$$

logo, definindo, para cada $r \in \mathbb{N}, \phi_{r}: \mathbb{R} \rightarrow X$ por $\phi_{r}(t):=\zeta_{r}^{(1)}\left(t+s_{r}^{\prime}\right), t \in \mathbb{R}$, como antes, podemos supor a existência de uma solução $\zeta^{(2)}: \mathbb{R} \rightarrow X$ que não está contida em $\Xi_{\sigma\left(i_{1}\right)}$ de maneira que $\phi_{r} \underset{r \rightarrow \infty}{\rightarrow} \zeta^{(2)}$ uniformemente sobre compactos de $\mathbb{R}$, com

$$
\lim _{t \rightarrow \infty} d\left(\zeta^{(2)}(t), \Xi_{\sigma\left(i_{1}\right)}\right)=0
$$

e, novamente pelo fato de que $(T(\cdot), \boldsymbol{\Xi})$ satisfaz $(G 1)$ e pelo que provamos com relação a ordem determinada pela permutação $\sigma: I_{n} \rightarrow I_{n}$, conclui-se a existência de um elemento $\Xi_{\sigma\left(i_{2}\right)} \in \boldsymbol{\Xi}$, com $i_{2}>i_{1}$, de modo que $\lim _{t \rightarrow-\infty} d\left(\zeta^{(2)}(t), \Xi_{\sigma\left(i_{2}\right)}\right)=0$.

Agora, observando que o conjunto $\Xi$ é finito, este raciocínio deverá parar em um número finito de etapas e na etapa final estaremos obrigados a ter, ou uma estrutura homoclínica em $\mathcal{A}$ associada a $\boldsymbol{\Xi}$ ou contradizer a ordem definida por $\sigma: I_{n} \rightarrow I_{n}$, as quais são duas situações impossíveis, terminando a prova de nossa afirmação.

Por outro lado, observemos que (4.3.7) também nos diz que para o $\delta_{0}>0$, obtido acima, tem-se para cada $j=1,2, \cdots, n-1$ que

$$
\left(\bigcup_{i=j+1}^{n} \Xi_{\sigma(i)}\right) \cap \mathcal{O}_{\delta_{0}}\left(A_{j}\right)=\varnothing
$$

Agora, afirmamos que para todo $j=1,2, \cdots, n$ e todo $\delta \in\left(0, \delta_{0}\right)$ existe $\delta^{\prime} \in(0, \delta)$ com

$$
\gamma^{+}\left(\mathcal{O}_{\delta^{\prime}}\left(A_{j}\right)\right) \subset \mathcal{O}_{\delta}\left(A_{j}\right)
$$


Com efeito, em primeiro lugar, o caso em que $j=n$ vemos que $A_{n}=\bigcup_{i=1}^{n} W^{u}\left(\Xi_{\sigma(i)}\right)=$ $\mathcal{A}$ e portanto a afirmação resulta verdadeira graças ao Lema 4.1.2.

Suponhamos agora que a afirmação seja falsa para um certo $j=1,2, \cdots, n-1$, logo existem $\delta \in\left(0, \delta_{0}\right)$ e sequências $\left(t_{k}\right)_{k \in \mathbb{N}}$ de números positivos com $t_{k} \rightarrow \infty$ e $\left(x_{k}\right)_{k \in \mathbb{N}}$ de pontos de $X$ tais que

$$
\begin{gathered}
d\left(x_{k}, A_{j}\right)<\frac{1}{k}, \\
d\left(T\left(t_{k}\right) x_{k}, A_{j}\right)=\delta .
\end{gathered}
$$

e

$$
d\left(T(t) x_{k}, A_{j}\right)<\delta \text { sempre que } t \in\left[0, t_{k}\right) .
$$

Daí, por argumentos usuais, obtém-se uma solução global $\xi_{0}: \mathbb{R} \rightarrow X$ para $T(\cdot)$ satisfazendo

$$
d\left(\xi_{0}(t), A_{j}\right) \leq \delta \text { para todo } t \leq 0
$$

com

$$
d\left(\xi_{0}(0), A_{j}\right)=\delta
$$

Como $(T(\cdot), \boldsymbol{\Xi})$ satisfaz $(G 1)$ existe $\Xi_{\sigma(i)} \in \boldsymbol{\Xi}$ tal que

$$
\lim _{t \rightarrow-\infty} d\left(\xi_{0}(t), \Xi_{\sigma(i)}\right)=0
$$

e como $\delta \in\left(0, \delta_{0}\right)$, onde $\delta_{0}$ satisfaz (4.3.11), levando em conta (4.3.12), resulta que $i \leq j$, mostrando que $\xi_{0}(0) \in W^{u}\left(\Xi_{\sigma(i)}\right) \subset A_{j}$, o que contradiz (4.3.13) e por isso nossa segunda afirmação também é correta.

Nestas condições, dado qualquer $j=1,2, \cdots, n$, levando em conta que nossa segunda afirmação é certa, fixemos $\delta \in\left(0, \delta_{0}\right)$ e $\delta^{\prime} \in(0, \delta)$ de modo que

$$
\gamma^{+}\left(\mathcal{O}_{\delta^{\prime}}\left(A_{j}\right)\right) \subset \mathcal{O}_{\delta}\left(A_{j}\right)
$$

o que implica

$$
\omega\left(\mathcal{O}_{\delta^{\prime}}\left(A_{j}\right)\right) \subset \overline{\mathcal{O}_{\delta}\left(A_{j}\right)}
$$

logo se $x \in \omega\left(\mathcal{O}_{\delta^{\prime}}\left(A_{j}\right)\right)$, devido a invariância de $\omega\left(\mathcal{O}_{\delta^{\prime}}\left(A_{j}\right)\right)$, existe uma solução global $\xi: \mathbb{R} \rightarrow X \operatorname{com} \xi(0)=x$ de modo que $\xi(t) \in \omega\left(\mathcal{O}_{\delta^{\prime}}\left(A_{j}\right)\right)$ para todo real $t$. Logo $\xi(t) \in \overline{\mathcal{O}_{\delta}\left(A_{j}\right)} \subset \mathcal{O}_{\delta_{0}}\left(A_{j}\right)$ para todo $t$ real, donde vemos, segundo a escolha de $\delta_{0}$ em conjunto com a propriedade $(G 1)$, que obrigatoriamente $\xi(t) \in A_{j}$ para todo $t$ real, e em particular $x=\xi(0) \in A_{j}$, provando que $\omega\left(\mathcal{O}_{\delta^{\prime}}\left(A_{j}\right)\right) \subset A_{j}$.

Reciprocamente, simplesmente pela invariância de $A_{j}$, concluímos que $A_{j} \subset \omega\left(\mathcal{O}_{\delta^{\prime}}\left(A_{j}\right)\right)$, mostrando que $A_{j}=\omega\left(\mathcal{O}_{\delta^{\prime}}\left(A_{j}\right)\right)$, ou seja, que $A_{j}$ é um atrator local para $T(\cdot)$ em $X$. 
Finalmente, para provar que para todo $j=1,2, \cdots, n$ tem-se a igualdade $\Xi_{\sigma(j)}=$ $A_{j} \cap A_{j-1}^{*}$, observemos que para cada $j$

$$
A_{j}=\bigcup_{i=1}^{j} W^{u}\left(\Xi_{\sigma(i)}\right)
$$

portanto, dado $x \in A_{j} \cap A_{j-1}^{*}$ obtém-se a existência de uma solução global $\xi: \mathbb{R} \rightarrow X$, passando pelo ponto $x$, que deve cumprir

$$
\lim _{t \rightarrow-\infty} d\left(\xi(t), \bigcup_{i=1}^{j} \Xi_{\sigma(i)}\right)=0 \text { e } \lim _{t \rightarrow \infty} d\left(\xi(t), \bigcup_{i=j}^{n} \Xi_{\sigma(i)}\right)=0,
$$

sendo a convergência indicada à direita devida à propriedade $(G 1)$ em conjunto com o fato de que $x \in A_{j-1}^{*}$ e a da esquerda devida ao fato de que $x \in \bigcup_{i=1}^{j} W^{u}\left(\Xi_{\sigma(i)}\right)$.

Pela maneira com a qual estão ordenados os elementos da $n$-upla $\mathbf{D}$, de acordo com a permutação $\sigma: I_{n} \rightarrow I_{n}$, a única maneria de (4.3.14) ser verdadeiro é quando

$$
\lim _{t \rightarrow-\infty} d\left(\xi(t), \Xi_{\sigma(j)}\right) \text { e } \lim _{t \rightarrow \infty} d\left(\xi(t), \Xi_{\sigma(j)}\right)
$$

mostrando que $x \in \Xi_{\sigma(j)}$, porque $\xi$ não pode ser uma solução homoclínica, assim $A_{j} \cap A_{j-1}^{*} \subset \Xi_{\sigma(j)}$.

Reciprocamente, se $x \in \Xi_{\sigma(j)}$ então, como $\Xi_{\sigma(j)}$ é fechado e invariante, segue-se que $\omega(x) \subset \Xi_{\sigma(j)}$, donde, em virtude da separação dada em (4.3.11), $\omega(x) \cap A_{j-1}=\varnothing$, portanto $x \in A_{j-1}^{*}$ e como $\Xi_{\sigma(j)} \subset A_{j}$ deduz-se que $x \in A_{j}$, completando a prova do teorema.

Terminamos esta seção provando a proposição abaixo, que é de grande ajuda quando usamos a decomposição de Morse, dada no teorema anterior, com a função de Lyapunov dos pares atrator-repulsor correspondentes, a fim de construir uma função de Lyapunov para os semigrupos de tipo gradiente, como explicamos na próxima seção.

Proposição 4.3.4. Seja $\{T(t): t \geq 0\}$ um semigrupo em um espaço métrico $X$ possuindo atrator global $\mathcal{A}$. Então, seja qual for a decomposição de Morse $\mathbf{D}=\left(M_{1}, \cdots, M_{n}\right)$ de $\mathcal{A}$, com $M_{j}:=A_{j} \cap A_{j-1}^{*}$ para $j=1, \cdots, n$, tem-se

$$
\bigcup_{j=1}^{n} M_{j}=\bigcap_{j=0}^{n}\left(A_{j} \cup A_{j}^{*}\right)
$$

onde

$$
\varnothing=A_{0} \subset A_{1} \subset \cdots \subset A_{n-1} \subset A_{n}=\mathcal{A},
$$


é a cadeia crescente de atratores locais e

$$
\varnothing=A_{n}^{*} \subset A_{n-1}^{*} \subset \cdots \subset A_{1}^{*} \subset A_{0}^{*}=\mathcal{A}
$$

é a cadeia decrescente de seus repulsores correspondentes.

Demonstração: Com efeito, se $x \in \bigcup_{j=1}^{n} M_{j}$, consideremos $j_{0} \in\{1,2, \cdots, n\}$ tal que $x \in M_{j_{0}}=A_{j_{0}} \cap A_{\left(j_{0}-1\right)}^{*}$, então $x \in A_{j_{0}} \subset A_{\left(j_{0}+1\right)} \subset \cdots \subset A_{n}$ e $x \in A_{\left(j_{0}-1\right)}^{*} \subset A_{\left(j_{0}-2\right)}^{*} \subset$ $\cdots \subset A_{0}^{*}, \log \mathrm{O}$

$$
\begin{gathered}
x \in\left(\bigcap_{j=j_{0}}^{n} A_{j}\right) \cap\left(\bigcap_{j=0}^{j_{0}-1} A_{j}^{*}\right) \subset\left[\bigcap_{j=j_{0}}^{n}\left(A_{j} \cup A_{j}^{*}\right)\right] \cap\left[\bigcap_{j=0}^{j_{0}-1}\left(A_{j} \cup A_{j}^{*}\right)\right]= \\
\bigcap_{j=0}^{n}\left(A_{j} \cup A_{j}^{*}\right),
\end{gathered}
$$

demonstrando a inclusão $\bigcup_{j=1}^{n} M_{j} \subset \bigcap_{j=0}^{n}\left(A_{j} \cup A_{j}^{*}\right)$.

Reciprocamente, se $x \in \bigcap_{j=0}^{n}\left(A_{j} \cup A_{j}^{*}\right)$, pondo $K:=\{0,1,2, \cdots, n\}$, definamos

$$
I:=\left\{i \in K: x \in A_{i}\right\} \text { e } J:=\left\{i \in K: x \in A_{i}^{*}\right\}
$$

Daí resulta imediatamente que $I \cup J=\{0,1,2, \cdots, n\}$ com $I \cap J=\varnothing$.

Claramente, os dois conjuntos $I$ e $J$ são não vazios, então pondo $i:=\min I$ resulta que $I=\{i, i+1, i+2, \cdots, n\}$ e $J=\{0,1, \cdots, i-1\}$, logo, em particular, $x \in A_{i}$ e $x \in A_{i-1}^{*}$, isto é, $x \in A_{i} \cap A_{i-1}^{*}=M_{i}$, donde conclui-se a inclusão que faltava $\bigcap_{j=0}^{n}\left(A_{j} \cup A_{j}^{*}\right) \subset \bigcup_{j=1}^{n} M_{j}$, completando a prova da proposição.

\subsection{Equivalência entre as noções de semigrupos gradi- ente e de tipo gradiente}

Terminemos o capítulo estabelecendo nosso principal resultado no contexto dos semigrupos, que consiste em provar a equivalência entre os dois conceitos de sistemas dinâmicos autônomos que estudamos até agora, isto é, a equivalência entre as noções de sistemas gradientes e sistemas de tipo gradiente.

O fato surpreendente nesta construção é que a função de Lyapunov é obtida como uma consequência da dinâmica do sistema, evidenciando assim a "rigidez" que possui a 
dinâmica dos semigrupos gradientes. Além disso, também neste capítulo, provamos que a função de Lyapunov pode ser construída de modo que seja diferenciável ao longo de soluções e, mais tarde, no próximo capítulo, que esta função é, sob condições bastante razoáveis, estável por perturbação.

Teorema 4.4.1. Seja $\{T(t): t \geq 0\}$ um semigrupo num espaço métrico $X$ possuindo atrator global $\mathcal{A}$ e família disjunta $\Xi=\left\{\Xi_{1}, \Xi_{2}, \cdots, \Xi_{n}\right\}$ de conjuntos invariantes isolados limitados.

Então, $\{T(t): t \geq 0\}$ é um semigrupo gradiente generalizado com respeito à $\boldsymbol{\Xi}$ se, e somente se, é de tipo gradiente generalizado com respeito à $\boldsymbol{\Xi}$.

Demonstração: Como vimos na Proposição 2.2.2, todo semigrupo gradiente generalizado é de tipo gradiente generalizado com respeito à mesma família de invariantes isolados, então precisamos apenas provar que todo semigrupo de tipo gradiente é, efetivamente, um semigrupo gradiente.

Com efeito, suponhamos que $(T(\cdot), \boldsymbol{\Xi})$ seja um semigrupo de tipo gradiente e seja $\sigma: I_{n} \rightarrow I_{n}$ uma permutação do conjunto $I_{n}$ de maneira que a $n$-upla ordenada

$$
\mathbf{D}:=\left(\Xi_{\sigma(1)}, \Xi_{\sigma(2)}, \cdots, \Xi_{\sigma(n)}\right)
$$

determine, segundo o Teorema 4.3.3, uma decomposição de Morse para o atrator $\mathcal{A}$.

Nestas condições, sejam

$$
\varnothing=A_{0} \subset A_{1} \subset \cdots \subset A_{n-1} \subset A_{n}=\mathcal{A},
$$

a cadeia de atratores locais e

$$
\varnothing=A_{n}^{*} \subset A_{n-1}^{*} \subset \cdots \subset A_{1}^{*} \subset A_{0}^{*}=\mathcal{A}
$$

a de seus repulsores correspondentes, de modo que para cada $j=1,2, \cdots, n$

$$
\Xi_{\sigma(j)}=A_{j} \cap A_{j-1}^{*} \text {. }
$$

Agora, sejam, para cada $j=0,1,2, \cdots, n, f_{j}: X \rightarrow \mathbb{R}$ a função de Lyapunov associada ao par atrator-repulsor $\left(A_{j}, A_{j}^{*}\right)$, de acordo com a Proposição $4.2 .2^{3}$, e definamos $V: X \rightarrow \mathbb{R}$ como

$$
V(x):=\sum_{j=0}^{n} f_{j}(x), x \in X .
$$

Afirmamos que $V: X \rightarrow \mathbb{R}$, assim definida, cumpre todas as condições da Definição 3.1.1.

\footnotetext{
${ }^{3}$ Nos casos especiais $\left(A_{0}, A_{0}^{*}\right)=(\varnothing, \mathcal{A})$ e $\left(A_{n}, A_{n}^{*}\right)=(\mathcal{A}, \varnothing)$, convencionamos que $f_{0}(x):=1+h(x)$ e $f_{n}(x):=h(x)$, para todo $x \in X$, sendo $h: X \rightarrow \mathbb{R}$ a função dada no Lema 4.2.1
} 
De fato, em primeiro lugar, é evidente que $V$ é contínua, por ser uma soma finita de funções contínuas e é também não crescente ao longo de soluções de $T(\cdot)$ porque assim o são cada uma das funções $f_{j}: X \rightarrow \mathbb{R}$.

Agora, suponhamos que $x \in X$ seja tal que $V(T(t) x)=V(x)$ para todo $t \geq 0$. Então, como cada $f_{j}: X \rightarrow \mathbb{R}$ é não crescente ao longo de soluções de $T(\cdot)$, esta situação implica que, para cada $j=0,1,2, \cdots, n, f_{j}(T(t) x)=f_{j}(x)$ sempre que $t \geq 0$ e, portanto, a Proposição 4.2 .2 nos diz que para cada $j=0,1,2, \cdots, n, x$ deve pertencer a $A_{j} \cup A_{j}^{*}$, consequentemente $x \in \bigcap_{j=0}^{n}\left(A_{j} \cup A_{j}^{*}\right)$, mas a Proposição 4.3.4 afirma que $\bigcap_{j=0}^{n}\left(A_{j} \cup A_{j}^{*}\right)=\bigcup_{j=1}^{n} \Xi_{j}$, o que nos dá $x \in \Xi_{j}$ para algum $j=1,2, \cdots, n$, estabelecendo a propriedade (iii) da Definição 3.1.1.

Finalmente, para provar a propriedade $(i v)$ fixemos um conjunto invariante qualquer $\Xi_{\sigma(k)} \in \boldsymbol{\Xi}$ e um ponto $x \in \Xi_{\sigma(k)}$. Devido ao fato de que $\Xi_{\sigma(k)}=A_{k} \cap A_{k-1}^{*}$ junto com (4.4.16) e (4.4.17), tem-se

$$
x \in A_{k} \subset A_{k+1} \subset \cdots \subset A_{n}
$$

$\mathrm{e}$

$$
x \in A_{k-1}^{*} \subset A_{k-2}^{*} \subset \cdots \subset A_{0}^{*},
$$

donde segue, também pela Proposição 4.2.2, que $f_{j}(x)=0$ se $j=k, k+1, \cdots, n$ e $f_{j}(x)=1$ se $j=0,1, \cdots, k-2, k-1$, e então

$$
V(x)=\sum_{j=0}^{n} f_{j}(x)=\sum_{j=0}^{k-1} f_{j}(x)+\sum_{j=k}^{n} f_{j}(x)=\sum_{j=0}^{k-1} 1+\sum_{j=k}^{n} 0=k,
$$

completando a demonstração.

Observação 4.4.2. É importante notarmos que a função de Lyapunov $V: X \rightarrow \mathbb{R}$ obtida no teorema anterior é tal que, para $k=1,2, \cdots, n, V(x)=k$ qualquer que seja $x \in \Xi_{\sigma(k)}$.

Provamos a seguir, com base na Proposição 4.2.2 e utilizando-se algumas idéias encontradas em [12], que é possível obter uma função de Lyapunov para um par atratorrepulsor, que é diferenciável ao longo de soluções de $T(\cdot)$, donde, repetindo todos os passos da prova do teorema anterior, concluímos a existência de uma função de Lyapunov para os semigrupos de tipo gradiente que é diferenciável ao longo de soluções. 
Proposição 4.4.3. Seja $\left(A, A^{*}\right)$ um par atrator-repulsor para um semigrupo $\{T(t)$ : $t \geq 0\}$ em um espaço métrico $(X, d)$ possuindo atrator global $\mathcal{A}$.

Então, existe uma função $g: X \rightarrow \mathbb{R}$ satisfazendo todas as propriedades enunciadas na Proposição 4.2.2 e, além disso, sendo decrescente ao longo de soluções de $T(\cdot)$ por pontos de $X \backslash\left(A \cup A^{*}\right)$ e diferenciável ao longo de soluções.

Demonstração: Com efeito, seja $g: X \rightarrow \mathbb{R}$ a função dada por

$$
g(x):=\int_{0}^{\infty} e^{-t} f(T(t) x) d t, x \in X
$$

onde $f: X \rightarrow \mathbb{R}$ é a função de Lyapunov associada ao par $\left(A, A^{*}\right)$ dada pela Proposição 4.2 .2 .

Observemos que para todo $x \in X$ esta integral é convergente, porque a função $f$ é não crescente ao longo de soluções e $\int_{0}^{\infty} e^{-t} d t<\infty$, donde a função $g: X \rightarrow \mathbb{R}$ está bem definida.

Provemos que $g: X \rightarrow \mathbb{R}$ possui as propriedades enunciadas na proposição.

Em primeiro lugar, a continuidade de $g$ pode-se provar assim:

Por um lado, usando a definição de $f$, vemos que $f(x) \leq 1+h(x)$ para todo $x \in X$, onde $h: X \rightarrow \mathbb{R}$ é a função definida no Lema 4.2.1. Dado $x_{0} \in X$, seja $B$ uma vizinhança limitada de $x_{0}$ e, usando o fato de que $T(\cdot)$ possui atrator global, seja $t_{1}>0$ de modo que $\gamma_{t_{1}}^{+}(B) \subset \mathcal{O}_{1}(\mathcal{A})$. Então pondo

$$
M_{B}:=\sup \left\{f(T(t) x): x \in B, t \geq t_{1}\right\}
$$

resulta que $M_{B}$ é finito, podendo ser nulo.

Por outro lado, dado $\varepsilon>0$, graças à convergência da integral $\int_{0}^{\infty} e^{-t} d t$, escolhamos $t_{2} \geq t_{1}$ tal que

$$
\int_{t_{2}}^{\infty} e^{-t} d t<\frac{\varepsilon}{4\left(M_{B}+1\right)}
$$

Agora, como a família $\left\{T(t): t \in\left[0, t_{2}\right]\right\}$ é equicontínua no ponto $x_{0}$ e $f$ é contínua, é fácil ver que existe $\delta>0$ tal que se $x \in X$ satisfaz $d\left(x, x_{0}\right)<\delta$ então

$$
\int_{0}^{t_{2}} e^{-t}\left|f(T(t) x)-f\left(T(t) x_{0}\right)\right| d t \leq \frac{\varepsilon}{2}
$$

donde, levando em conta (4.4.18) e a definição de $M_{B}$, vemos que para todo $x \in B$ com $d\left(x, x_{0}\right)<\delta$ verifica-se

$$
\begin{gathered}
\left|g(x)-g\left(x_{0}\right)\right| \leq \\
\int_{0}^{t_{2}} e^{-t}\left|f(T(t) x)-f\left(T(t) x_{0}\right)\right| d t+2 M_{B} \int_{t_{2}}^{\infty} e^{-t} d t \leq \frac{\varepsilon}{2}+\frac{\varepsilon}{2}=\varepsilon,
\end{gathered}
$$


o que justifica a continuidade de $g$ no ponto $x_{0}$, terminando a prova de continuidade de $g$ nos pontos de $X$.

Claramente, $g: X \rightarrow \mathbb{R}$ é não crescente ao longo de soluções de $\{T(t): t \geq 0\}$, simplesmente porque assim o é $f: X \rightarrow \mathbb{R}$ e o processo de integração é monôtono crescente.

Agora, se $x \in A$ temos que $T(t) x \in A$ para todo $t \geq 0$, logo, pela correspondente propriedade de $f, f(T(t) x)=0$ para todo $t \geq 0$, mostrando que $g(x)=0$. Analogamente, se $x \in A^{*}$ temos que $T(t) x \in A^{*}$ para todo $t \geq 0$, portanto $f(T(t) x)=1$ para todo $t \geq 0$, implicando que $g(x)=\int_{0}^{\infty} e^{-t} \cdot 1 d t=1$. Destes fatos vemos que $g(A)=\{0\}$ e $g\left(A^{*}\right)=\{1\}$.

Reciprocamente, se $x \in X$ é tal que $0=g(x)=\int_{0}^{\infty} e^{-t} f(T(t) x) d t$, então $e^{-t} f(T(t) x)=0$ para todo $t \geq 0$ o que dá $f(T(t) x)=0$ para todo $t \geq 0$, logo $x \in A$, graças propriedades da função $f$ dadas na Proposição 4.2.2, mostrando que $g^{-1}(0)=A$. Se agora $x \in \mathcal{A}$ é tal que $g(x)=1$, deve existir $t^{\prime} \geq 0$ tal que $f\left(T\left(t^{\prime}\right) x\right)=1$, pois, de outro modo, $f(T(t) x)<1$ para todo $t \geq 0$, e por isto, $g(x)=\int_{0}^{\infty} e^{-t} f(T(t) x) d t<\int_{0}^{\infty} e^{-t} \cdot 1 d t=1$, contradizendo a escolha de $x$. Daí, se $t^{\prime} \geq 0$ for tal que $f\left(T\left(t^{\prime}\right) x\right)=1$, teremos, pelas propriedades da função $f$, que $T\left(t^{\prime}\right) x \in A^{*}$, donde $x \in A^{*}\left(\right.$ pois $\omega(x)=\omega\left(T\left(t^{\prime}\right) x\right)$ ) e $\operatorname{assim} g^{-1}(1) \cap \mathcal{A} \subset A^{*}$, ou seja, $g^{-1}(1) \cap \mathcal{A}=A^{*}$, provando a propriedade (iii) da Proposição 4.2.2.

Dado $x \in X \backslash\left(A \cup A^{*}\right)$ provemos que $f$ é estritamente decrescente ao longo da solução por $x$. Com efeito, dado $t>0$ temos que

$$
\begin{gathered}
g(T(t) x)-g(x)=\int_{0}^{\infty} e^{-s} f(T(s) T(t) x) d s-\int_{0}^{\infty} e^{-s} f(T(s) x) d s \\
=\int_{0}^{\infty} e^{-s}[f(T(s+t) x)-f(T(s) x)] d s,
\end{gathered}
$$

donde vemos que se $g(T(t) x)-g(x)=0$ então, como $f(T(s+t) x)-f(T(s) x) \leq 0$ para todo $s \geq 0$, obrigatoriamente tem-se $f(T(s+t) x)-f(T(s) x)=0$ para todo $s \geq 0$. Em particular, $f(T(t) x)=f(x)$ e por isto, $f(T(t) x)=f(T(s) x)=f(x)$ para todo $s \in[0, t]$. Repetindo este raciocínio, do fato de que $t>0$, conclui-se que $f(T(s) x)=f(x)$ para todo $s \geq 0$ o que obriga, pela Proposição 4.2.2, $x \in\left(A \cup A^{*}\right)$, que é uma contradição com a maneira com a qual $x$ foi escolhido e daí conclui-se que $g(T(t) x)<g(x)$.

Finalmente, para ver a diferenciabilidade ao longo de soluções, dados $x \in X, t_{0}>0$ e $t \in \mathbb{R}$ com $t \neq 0$ e $t+t_{0}>0$, um cálculo simples nos dá

$$
\frac{g\left(T\left(t_{0}+t\right) x\right)-g\left(T\left(t_{0}\right) x\right)}{t}=
$$




$$
\frac{e^{t_{0}}}{t}\left[\left(e^{t}-1\right) \int_{t_{0}+t}^{\infty} e^{-s} f(T(s) x) d s-\int_{t_{0}}^{t_{0}+t} e^{-s} f(T(s) x) d s\right],
$$

donde vê-se, fazendo $t \rightarrow 0$, que o quociente anterior converge para

$$
e^{t_{0}} \int_{t_{0}}^{\infty} e^{-s} f(T(s) x) d s-f\left(T\left(t_{0}\right) x\right) \leq 0,
$$

completando a demonstração.

Os resultados obtidos neste capítulo nos permite enunciar condições suficientes que assegurem a estabilidade dos semigrupos gradientes por perturbação.

Corolário 4.4.4. Seja $\left\{T_{\eta}(\cdot): t \geq 0\right\}_{\eta \in[0,1]}$ uma família de semigrupos num espaço métrico $X$ satisfazendo as condições do Teorema de Carvalho e Langa. Então, existe $\eta_{0}>0$ tal que para todo $\eta \in\left[0, \eta_{0}\right]$ o semigrupo $T_{\eta}(\cdot)$ é um semigrupo gradiente com respeito a família $\boldsymbol{\Xi}_{\eta}$, possuindo função de Lyapunov diferenciável ao longo de soluções. 


\section{$-\frac{\pi}{5}$ \\ Estabilidade da função de Lyapunov}

Neste capítulo, estudamos a estabilidade por perturbação da função de Lyapunov dos semigrupos de tipo gradiente que construímos no capítulo anterior. Para isso, é necessário entender as noções de semicontinuidade superior e inferior como definimos a seguir.

\subsection{Continuidade da família de atratores}

Definição 5.1.1. Seja $\left(A_{\eta}\right)_{\eta \in[0,1]}$ uma família de subconjuntos em um espaço métrico $X$ com distância $d: X \times X \rightarrow \mathbb{R}$.

Diz-se que esta família é semicontínua superiormente (s.c.s.) em $\eta=0$ quando

$$
\lim _{\eta \rightarrow 0^{+}} \operatorname{dist}\left(A_{\eta}, A_{0}\right)=0 .
$$

Por outro lado, diz-se que ela é semicontinua inferiormente (s.c.i.) em $\eta=0$ quando

$$
\lim _{\eta \rightarrow 0^{+}} \operatorname{dist}\left(A_{0}, A_{\eta}\right)=0 .
$$

Finalmente, $\left(A_{\eta}\right)_{\eta \in[0,1]}$ diz-se contínua em $\eta=0$ quando é semicontínua superior $e$ inferiormente em $\eta=0$, ou em símbolos, quando

$$
\lim _{\eta \rightarrow 0^{+}} d_{H}\left(A_{\eta}, A_{0}\right)=0 .
$$

Dadas estas definições é possível estender, com demonstração semelhante, a conclusão do Lema 4.1.2 para famílias de atratores locais, mais precisamente tem-se o seguinte resultado. 
Lema 5.1.2. Seja $\left\{T_{\eta}(t): t \geq 0\right\}_{\eta \in(0,1]}$ uma família coletivamente assintoticamente compacta de semigrupos em um espaço métrico $X$ convergindo uniformemente sobre compactos ao semigrupo $\left\{T_{0}(t): t \geq 0\right\}$, de modo que, para cada $\eta \in[0,1],\left\{T_{\eta}(t): t \geq 0\right\}$ possui atrator global $\mathcal{A}_{\eta}$ com $\bigcup_{\eta \in[0,1]} \mathcal{A}_{\eta}$ limitada em $X$.

Seja também $\left(A_{\eta}\right)_{\eta \in[0,1]}$ uma família de subconjuntos de $X$ tal que cada $A_{\eta}$ é subconjunto de $\mathcal{A}_{\eta}$ e (supõe-se apenas que) $A_{0}$ é um atrator local para o semigrupo $\left\{T_{0}(t): t \geq 0\right\}$ com $\omega\left(\mathcal{O}_{\varepsilon}\left(A_{0}\right)\right)=A_{0}$, para um certo número positivo $\varepsilon$.

Nestas condições, se $\left(A_{\eta}\right)_{\eta \in[0,1]}$ é contínua em $\eta=0$, então dado $\delta \in(0, \varepsilon)$ existem $\delta^{\prime} \in(0, \delta)$ e $\eta_{0}>0$ tais que para todo $\eta \in\left[0, \eta_{0}\right]$ vale

$$
\gamma_{\eta}^{+}\left(\mathcal{O}_{\delta^{\prime}}\left(A_{\eta}\right)\right) \subset \mathcal{O}_{\delta}\left(A_{\eta}\right)
$$

onde, por simplicidade, para cada $\eta \in[0,1], \gamma_{\eta}^{+}\left(\mathcal{O}_{\delta^{\prime}}\left(A_{\eta}\right)\right)$ representa a semiórbita positiva do conjunto $\mathcal{O}_{\delta^{\prime}}\left(A_{\eta}\right)$ segundo o semigrupo $\left\{T_{\eta}(t): t \geq 0\right\}$.

Demonstração: Com efeito, se esta conclusão não fosse válida, existiriam $\delta>0 \mathrm{e}$ sequências $\left(x_{j}\right)_{j \in \mathbb{N}}$ em $X,\left(\eta_{j}\right)_{j \in \mathbb{N}}$ em $[0,1]$ e $\left(t_{j}\right)_{j \in \mathbb{N}}$ em $\mathbb{R}$ tais que, $\eta_{j} \underset{j \rightarrow \infty}{\rightarrow} 0^{+}, t_{j} \underset{j \rightarrow \infty}{\rightarrow} \infty$, $d\left(x_{j}, A_{\eta_{j}}\right)<\frac{1}{j}$ para todo $j$,

$$
d\left(T_{\eta_{j}}(t) x_{j}, A_{\eta_{j}}\right)<\delta \text { para todo } t \in\left[0, t_{j}\right) \text { e todo } j \in \mathbb{N}
$$

$\mathrm{e}$

$$
d\left(T_{\eta_{j}}\left(t_{j}\right) x_{j}, A_{\eta_{j}}\right)=\delta \text { para todo } j \in \mathbb{N} .
$$

Então, definindo, para cada natural $j, \xi_{j}:\left[-t_{j}, \infty\right) \rightarrow X$ por $\xi_{j}(t):=T_{\eta_{j}}\left(t+t_{j}\right) x_{j}$, usando as hipóteses de compacidade assintótica coletiva e de convergência uniforme em compactos, do Lema 3.2.3 seguiria a existência de uma solução global limitada $\xi_{0}: \mathbb{R} \rightarrow X$ para o semigrupo $\left\{T_{0}(t): t \geq 0\right\}$ e uma subsequência de $\left(\xi_{j}\right)_{j \in \mathbb{N}}$, que indicaríamos com a mesma notação, tal que para todo real $t, \xi_{0}(t)=\lim _{j \rightarrow \infty} \xi_{j}(t)$.

Por outro lado, dado $t<0$ para todo $j$ suficientemente grande teríamos

$$
d\left(\xi_{j}(t), A_{0}\right) \leq d\left(\xi_{j}(t), A_{\eta_{j}}\right)+\operatorname{dist}\left(A_{\eta_{j}}, A_{0}\right),
$$

donde obteríamos, pela semicontinuidade superior de $\left(A_{\eta}\right)_{\eta \in[0,1]}$, que para todo $t<0$

$$
d\left(\xi_{0}(t), A_{0}\right) \leq \delta
$$

e de $\delta=d\left(\xi_{j}(0), A_{\eta_{j}}\right) \leq d\left(\xi_{j}(0), A_{0}\right)+\operatorname{dist}\left(A_{0}, A_{\eta_{j}}\right)$, pela semicontinuidade inferior de $\left(A_{\eta}\right)_{\eta \in[0,1]}$, obteríamos $d\left(\xi_{0}(0), A_{0}\right)=\delta$.

Mas, como $\delta<\varepsilon, A_{0}$ atrairia o conjunto $K:=\left\{\xi_{0}(t): t \leq 0\right\}$, contradizendo $d\left(\xi_{0}(0), A_{0}\right)=\delta$ e completando a prova. 
A seguir provamos que a semicontinuidade superior da família de repulsores pode ser obtida como uma consequência da semicontinuidade inferior dos atratores locais correspondentes.

Observemos que o problema dual, ou seja, o problema de se deduzir a semicontuidade inferior da família dos repulsores a partir da superior dos atratores, é uma questão para a qual ainda não sabemos a resposta.

Lema 5.1.3. Seja $\left\{T_{\eta}(t): t \geq 0\right\}_{\eta \in[0,1]}$ uma família coletivamente assintoticamente compacta de semigrupos num espaço métrico $X$ convergindo uniformemente em compactos ao semigrupo $\left\{T_{0}(t): t \geq 0\right\}$, tal que, para cada $\eta \in[0,1],\left\{T_{\eta}(t): t \geq 0\right\}$ possui atrator global $\mathcal{A}_{\eta}$ de modo que $\bigcup_{\eta \in[0,1]} \mathcal{A}_{\eta}$ é limitada em $X$.

Seja também, $\left(A_{\eta}\right)_{\eta \in[0,1]}$ uma família de subconjuntos de $X$ tal que cada $A_{\eta}$ é um atrator local para o semigrupo $\left\{T_{\eta}(t): t \geq 0\right\}$ e seja $\left(A_{\eta}^{*}\right)_{\eta \in[0,1]}$ a família de seus repulsores complementares correspondentes.

Suponhamos que existam $\mu>0$ e um indice $\tilde{\eta}>0$ tais que $d\left(A_{\eta}, A_{\eta}^{*}\right) \geq \mu$ sempre que $\eta \in[0, \tilde{\eta}]$, e além disso que a família $\left(\mathcal{A}_{\eta}\right)_{\eta \in[0,1]}$, dos atratores globais, seja contínua em $\eta=0$.

Nestas condições:

Se $\left(A_{\eta}\right)_{\eta \in[0,1]}$ é contínua em $\eta=0$, então $\left(A_{\eta}^{*}\right)_{\eta \in[0,1]}$ é semicontínua superiormente em $\eta=0$.

Demonstração: Com efeito, suponhamos que $\lim _{\eta \rightarrow 0^{+}} \operatorname{dist}\left(A_{0}, A_{\eta}\right)=0$ mas que não se tenha $\lim _{\eta \rightarrow 0^{+}} \operatorname{dist}\left(A_{\eta}^{*}, A_{0}^{*}\right)=0$. Daí resulta a existência de um $\varepsilon>0$ e uma sequência $\left(\eta_{j}\right)_{j \in \mathbb{N}}$ em $(0,1] \operatorname{com} \eta_{j} \underset{j \rightarrow \infty}{\rightarrow} 0^{+}$tais que

$$
\operatorname{dist}\left(A_{\eta_{j}}^{*}, A_{0}^{*}\right) \geq \varepsilon \text { para todo } j \in \mathbb{N} \text {. }
$$

Donde segue a existência de uma sequência $\left(x_{j}\right)_{j \in \mathbb{N}}$ de pontos de $X \operatorname{com} x_{j} \in A_{\eta_{j}}^{*} \subset$ $\mathcal{A}_{\eta_{j}}$ e $d\left(x_{j}, A_{0}^{*}\right)>\frac{\varepsilon}{2}$ para todo $j$.

Como a família de atratores globais $\left(\mathcal{A}_{\eta}\right)_{\eta \in[0,1]}$ é contínua em $\eta=0$, podemos supor, passando a uma subsequência caso seja necessário, que $x_{j} \underset{j \rightarrow \infty}{\rightarrow} x_{0}$ para algum $x_{0} \in \mathcal{A}_{0}$, e então devemos ter $d\left(x_{0}, A_{0}^{*}\right) \geq \frac{\varepsilon}{2}$, o que nos dá $\omega\left(x_{0}\right) \subset A_{0}$.

Por um lado, seja $0<\delta<\frac{\mu}{2}$ e, pelo lema anterior, associado a ele consideremos $\delta^{\prime} \in(0, \delta)$ e $\eta_{0} \in(0, \tilde{\eta}]$ tais que

$$
\gamma_{\eta}^{+}\left(\mathcal{O}_{\delta^{\prime}}\left(A_{\eta}\right)\right) \subset \mathcal{O}_{\delta}\left(A_{\eta}\right)
$$

sempre que $\eta \in\left[0, \eta_{0}\right]$. 
Como $\omega\left(x_{0}\right) \subset A_{0}$, existe $t_{0}>0$ tal que $T_{0}\left(t_{0}\right) x_{0} \in \mathcal{O}_{\frac{\delta^{\prime}}{4}}\left(A_{0}\right)$.

Por outro, da semicontinuidade inferior de $\left(A_{\eta}\right)_{\eta \in[0,1]}$ obtém-se a existência de $\eta_{1} \in$ $\left(0, \eta_{0}\right]$ tal que para todo $\eta \in\left[0, \eta_{1}\right]$ tem-se

$$
A_{0} \subset \mathcal{O}_{\frac{\delta^{\prime}}{2}}\left(A_{\eta}\right)
$$

Mas, da hipótese de convergência dos semigupos, conclui-se a existência de um $\eta_{2} \in\left(0, \eta_{1}\right]$ para o qual $T_{\eta_{j}}\left(t_{0}\right) x_{j} \in \mathcal{O}_{\frac{\delta^{\prime}}{2}}\left(A_{0}\right)$ para todo natural $j$ com $\eta_{j} \in\left[0, \eta_{2}\right]$.

Agora, de (5.1.2) resulta $\mathcal{O}_{\frac{\delta^{\prime}}{2}}\left(A_{0}\right) \subset \mathcal{O}_{\delta^{\prime}}\left(A_{\eta}\right)$ sempre que $\eta \in\left[0, \eta_{2}\right]$, donde se infere $T_{\eta_{j}}\left(t_{0}\right) x_{j} \in \mathcal{O}_{\delta^{\prime}}\left(A_{\eta_{j}}\right)$ toda vez que $\eta_{j} \in\left[0, \eta_{2}\right]$.

Finalmente, (5.1.1) nos diz que sempre que $\eta_{j} \in\left[0, \eta_{2}\right]$, temos

$$
\gamma_{\eta_{j}}^{+}\left(T_{\eta_{j}}\left(t_{0}\right) x_{j}\right) \subset \mathcal{O}_{\delta}\left(A_{\eta_{j}}\right)
$$

$\log \mathrm{O}$

$$
\omega_{\eta_{j}}\left(x_{j}\right) \subset \overline{\mathcal{O}_{\delta}\left(A_{\eta_{j}}\right)}
$$

sempre que $\eta_{j} \in\left[0, \eta_{2}\right]$ (onde, evidentemente, $\omega_{\eta_{j}}\left(x_{j}\right)$ representa o conjunto $\omega$-limite do ponto $x_{j}$ segundo o semigrupo $\left.\left\{T_{\eta_{j}}(t): t \geq 0\right\}\right)$, mas, da invariância de $A_{\eta_{j}}^{*}$ por $\left\{T_{\eta_{j}}(t): t \geq 0\right\}$, conclui-se que $\omega_{\eta_{j}}\left(x_{j}\right) \subset A_{\eta_{j}}^{*}$, contradizendo a escolha de $\delta<\frac{\mu}{2} \mathrm{e}$ provando assim o lema.

\subsection{Convergência das funções de Lyapunov}

Provemos agora a convergência uniforme sobre compactos das funções de Lyapunov dos semigrupos de tipo gradiente.

Proposição 5.2.1. Seja $\left\{T_{\eta}(t): t \geq 0\right\}_{\eta \in[0,1]}$ uma família coletivamente assintoticamente compacta de semigrupos num espaço métrico $X$ convergindo uniformemente em compactos ao semigrupo $\left\{T_{0}(t): t \geq 0\right\}$, de maneira que cada $\left\{T_{\eta}(t): t \geq 0\right\}$ possui atrator global $\mathcal{A}_{\eta}$.

Seja também, $\left(A_{\eta}\right)_{\eta \in[0,1]}$ uma família de subconjuntos de $X$ tal que cada $A_{\eta}$ é um atrator local para o semigrupo $\left\{T_{\eta}(t): t \geq 0\right\}$ e seja $\left(A_{\eta}^{*}\right)_{\eta \in[0,1]}$ a família de seus repulsores complementares correspondentes.

Suponhamos que a família de atratores locais $\left(A_{\eta}\right)_{\eta \in[0,1]}$, a família de seus repulsores $\left(A_{\eta}^{*}\right)_{\eta \in[0,1]}$ e a família $\left(\mathcal{A}_{\eta}\right)_{\eta \in[0,1]}$ de atratores globais sejam contínuas em $\eta=0$.

Finalmente, sejam, para cada $\eta \in[0,1], f_{\eta}: X \rightarrow \mathbb{R}$ a função de Lyapunov associada ao par atrator-repulsor $\left(A_{\eta}, A_{\eta}^{*}\right)$ como está definida na Proposição 4.2.2.

Nestas condições, $f_{\eta} \underset{\eta \rightarrow 0^{+}}{\rightarrow} f_{0}$ uniformemente sobre compactos de $X$.

Em outras palavras, se os pares atrator-repulsor se comportam continuamente, então as funções de Lyapunov destes pares também se comportam continuamente. 
Demonstração: Com efeito, em primeiro lugar, para cada $\eta \in[0,1]$ a função de Lyapunov $f_{\eta}: X \rightarrow \mathbb{R}$ é definida por

$$
f_{\eta}(x):=h_{\eta}(x)+k_{\eta}(x), x \in X
$$

onde para cada $x \in X$,

$$
\begin{gathered}
h_{\eta}(x):=\sup _{t \geq 0} d\left(T_{\eta}(t) x, \mathcal{A}_{\eta}\right) \\
k_{\eta}(x):=\sup _{t \geq 0} l_{\eta}\left(T_{\eta}(t) x\right) \text { e } l_{\eta}(x):=\frac{d\left(x, A_{\eta}\right)}{d\left(x, A_{\eta}\right)+d\left(x, A_{\eta}^{*}\right)} .
\end{gathered}
$$

Façamos a demonstração em três etapas:

Etapa 1: $l_{\eta} \underset{\eta \rightarrow 0^{+}}{\rightarrow} l_{0}$ uniformemente em $X$.

Com efeito, simplesmente aplicando a desigualdade triangular para a semidistância de Hausdorff vemos que para todo $\eta \in[0,1]$ e todo $x \in X$ tem-se

$$
\left|d\left(x, A_{\eta}\right)-d\left(x, A_{0}\right)\right| \leq d_{H}\left(A_{\eta}, A_{0}\right)
$$

e

$$
\left|d\left(x, A_{\eta}^{*}\right)-d\left(x, A_{0}^{*}\right)\right| \leq d_{H}\left(A_{\eta}^{*}, A_{0}^{*}\right) .
$$

Dados $\eta \in[0,1]$ e $x \in X$ escrevamos

$$
l_{\eta}(x)-l_{0}(x)=\frac{d\left(x, A_{\eta}\right) d\left(x, A_{0}^{*}\right)-d\left(x, A_{0}\right) d\left(x, A_{\eta}^{*}\right)}{\left[d\left(x, A_{\eta}\right)+d\left(x, A_{\eta}^{*}\right)\right]\left[d\left(x, A_{0}\right)+d\left(x, A_{0}^{*}\right)\right]}
$$

e agora, somando e subtraindo o termo $d\left(x, A_{0}\right) d\left(x, A_{0}^{*}\right)$, a igualdade acima fica

$$
l_{\eta}(x)-l_{0}(x)=\frac{\left[d\left(x, A_{\eta}\right)-d\left(x, A_{0}\right)\right] d\left(x, A_{0}^{*}\right)+d\left(x, A_{0}\right)\left[d\left(x, A_{0}^{*}\right)-d\left(x, A_{\eta}^{*}\right)\right]}{\left[d\left(x, A_{\eta}\right)+d\left(x, A_{\eta}^{*}\right)\right]\left[d\left(x, A_{0}\right)+d\left(x, A_{0}^{*}\right)\right]} .
$$

Como $d\left(A_{0}, A_{0}^{*}\right)>2 \mu$ para algum $\mu>0$, não é difícil provar, levando em conta a continuidade das famílias de atratores locais e seus repulsores, a existência de $\tilde{\eta} \in(0,1]$ de maneira que para todo $\eta \in[0, \tilde{\eta}]$ temos $d\left(A_{\eta}, A_{\eta}^{*}\right)>\mu$. Daí, pelo que fizemos acima, conclui-se que para todo $x \in X$ e todo $\eta \in[0, \tilde{\eta}]$

$$
\begin{gathered}
\left|l_{\eta}(x)-l_{0}(x)\right| \leq \\
\left|\frac{\left[d\left(x, A_{\eta}\right)-d\left(x, A_{0}\right)\right] d\left(x, A_{0}^{*}\right)+d\left(x, A_{0}\right)\left[d\left(x, A_{0}^{*}\right)-d\left(x, A_{\eta}^{*}\right)\right]}{\left[d\left(x, A_{\eta}\right)+d\left(x, A_{\eta}^{*}\right)\right]\left[d\left(x, A_{0}\right)+d\left(x, A_{0}^{*}\right)\right]}\right| \leq \\
\frac{1}{d\left(x, A_{\eta}\right)+d\left(x, A_{\eta}^{*}\right)}\left[d_{H}\left(A_{\eta}, A_{0}\right)+d_{H}\left(A_{\eta}^{*}, A_{0}^{*}\right)\right] \leq \\
\frac{1}{\mu}\left[d_{H}\left(A_{\eta}, A_{0}\right)+d_{H}\left(A_{\eta}^{*}, A_{0}^{*}\right)\right]
\end{gathered}
$$


portanto, para todo $x \in X$ e todo $\eta \in[0, \tilde{\eta}]$ de (5.2.3) e (5.2.4) segue-se que

$$
\left|l_{\eta}(x)-l_{0}(x)\right| \leq \frac{1}{\mu}\left[d_{H}\left(A_{\eta}, A_{0}\right)+d_{H}\left(A_{\eta}^{*}, A_{0}^{*}\right)\right]
$$

donde conclui-se a convergência $l_{\eta} \underset{\eta \rightarrow 0^{+}}{\rightarrow} l_{0}$ uniforme em $X$, simplesmente aplicando a continuidade das famílias $\left(A_{\eta}\right)_{\eta \in[0,1]}$ e $\left(A_{\eta}^{*}\right)_{\eta \in[0,1]}$ em $\eta=0$.

Etapa 2: $k_{\eta} \underset{\eta \rightarrow 0^{+}}{\rightarrow} k_{0}$ uniformemente em compactos de $X$.

Com efeito, dado um ponto qualquer $x \in X$ consideremos os seguintes três casos:

Caso $1: T_{0}(t) x \underset{t \rightarrow \infty}{\rightarrow} A_{0}$ com $l_{0}(x)>0$. Neste caso, escolhamos $0<\theta<l_{0}(x)$.

Pela continuidade da função $l_{0}: X \rightarrow \mathbb{R}$, seja $\sigma_{1}>0$ tal que $l_{0}\left(\mathcal{O}_{\sigma_{1}}(x)\right) \subset\left(\frac{\theta}{2}, 1\right]$ e, pela etapa anterior, $\eta_{0} \in(0,1]$ tal que $l_{\eta}\left(\mathcal{O}_{\sigma_{1}}(x)\right) \subset(\theta, 1]$ para todo $\eta \in\left[0, \eta_{0}\right]$.

Por um lado, usando mais uma vez a continuidade de $l_{0}: X \rightarrow \mathbb{R}$, dado $0<\alpha<\frac{\theta}{2}$ seja $\delta>0$ tal que $l_{0}\left(\mathcal{O}_{\delta}\left(A_{0}\right)\right) \subset[0, \alpha)$.

Por outro, sejam, segundo o Lema 5.1.2, $\delta^{\prime} \in\left(0, \frac{\delta}{2}\right)$ e $\eta_{1} \in\left(0, \eta_{0}\right]$ tais que para cada $\eta \in\left[0, \eta_{1}\right]$ temos

$$
\gamma_{\eta}^{+}\left(\mathcal{O}_{\delta^{\prime}}\left(A_{\eta}\right)\right) \subset \mathcal{O}_{\frac{\delta}{2}}\left(A_{\eta}\right)
$$

Agora, pela semicontinuidade inferior de $\left(A_{\eta}\right)_{\eta \in[0,1]}$ em $\eta=0$, seja $\eta_{2} \in\left(0, \eta_{1}\right]$ tal que para cada $\eta \in\left[0, \eta_{2}\right]$ vale

$$
A_{0} \subset \mathcal{O}_{\frac{\delta^{\prime}}{2}}\left(A_{\eta}\right)
$$

Pelo fato de que $T_{0}(t) x \underset{t \rightarrow \infty}{\rightarrow} A_{0}$, seja $t_{0}>0$ tal que $T_{0}\left(t_{0}\right) x \in \mathcal{O}_{\frac{\delta^{\prime}}{4}}\left(A_{0}\right) \mathrm{e}$ usando a continuidade do operador $T_{0}\left(t_{0}\right): X \rightarrow X$, escolhamos $\sigma_{2} \in\left(0, \sigma_{1}\right]$ de maneira que $T_{0}\left(t_{0}\right)\left(\mathcal{O}_{\sigma_{2}}(x)\right) \subset \mathcal{O}_{\frac{\delta^{\prime}}{4}}\left(A_{0}\right)$. Da hipótese de convergência dos semigrupos é possível encontrar $\sigma_{3} \in\left(0, \sigma_{2}\right]$ e $\eta_{3} \in\left(0, \eta_{2}\right]$ de modo que para todo $\eta \in$ $\left[0, \eta_{3}\right]$ tem-se $T_{\eta}\left(t_{0}\right)\left(\mathcal{O}_{\sigma_{3}}(x)\right) \subset \mathcal{O}_{\frac{\delta^{\prime}}{2}}\left(A_{0}\right)$, donde, levando em conta (5.2.6), obtémse $T_{\eta}\left(t_{0}\right)\left(\mathcal{O}_{\sigma_{3}}(x)\right) \subset \mathcal{O}_{\delta^{\prime}}\left(A_{\eta}\right)$ sempre que $\eta \in\left[0, \eta_{3}\right]$ e de (5.2.5) conclui-se que

$$
\gamma_{\eta}^{+}\left(T_{\eta}\left(t_{0}\right)\left(\mathcal{O}_{\sigma_{3}}(x)\right)\right) \subset \mathcal{O}_{\frac{\delta}{2}}\left(A_{\eta}\right) \text { sempre que } \eta \in\left[0, \eta_{3}\right]
$$

Observemos agora que, da convergência $l_{\eta} \underset{\eta \rightarrow 0^{+}}{\rightarrow} l_{0}$ uniforme em $X$, obtém-se $\eta_{4} \in$ $\left(0, \eta_{3}\right]$ com a propriedade de que para cada $\eta \in\left[0, \eta_{4}\right] l_{\eta}\left(\mathcal{O}_{\delta}\left(A_{0}\right)\right) \subset[0,2 \alpha)$ e da semicontinuidade superior de $\left(A_{\eta}\right)_{\eta \in[0,1]}$ em $\eta=0$ obtém-se a existência de $\eta_{5} \in\left(0, \eta_{4}\right]$ tal que toda vez que $\eta \in\left[0, \eta_{5}\right]$ tem-se $A_{\eta} \subset \mathcal{O}_{\frac{\delta}{2}}\left(A_{0}\right)$ e portanto $\mathcal{O}_{\frac{\delta}{2}}\left(A_{\eta}\right) \subset \mathcal{O}_{\delta}\left(A_{0}\right)$ sempre que $\eta \in\left[0, \eta_{5}\right]$. Logo $l_{\eta}\left(\mathcal{O}_{\frac{\delta}{2}}\left(A_{\eta}\right)\right) \subset[0,2 \alpha)$ sempre que $\eta \in\left[0, \eta_{5}\right]$, donde segue-se de (5.2.7) que para cada $\eta \in\left[0, \eta_{5}\right]$ e cada $z \in \mathcal{O}_{\sigma_{3}}(x) \subset \mathcal{O}_{\sigma_{1}}(x)$ vale

$$
\sup _{t \geq t_{0}} l_{\eta}\left(T_{\eta}(t) z\right) \leq 2 \alpha<\theta<l_{\eta}(z) \leq k_{\eta}(z)
$$


mostrando que $k_{\eta}(z)=\sup _{0 \leq t \leq t_{0}} l_{\eta}\left(T_{\eta}(t) z\right)$ para todo $\eta \in\left[0, \eta_{5}\right]$ e todo $z \in \mathcal{O}_{\sigma_{3}}(x)$.

Finalmente, dado $\varepsilon>0$, pela conclusão da Etapa 1 , existe $\eta_{6} \in\left(0, \eta_{5}\right]$ tal que para todo $z \in X$

$$
\left|l_{\eta}(z)-l_{0}(z)\right|<\frac{\varepsilon}{2} \text { sempre que } \eta \in\left[0, \eta_{6}\right]
$$

pela continuidade uniforme da função $l_{0}: X \rightarrow \mathbb{R}$, consideremos $\beta>0$ tal que para $z, z^{\prime} \in X \operatorname{com} d\left(z, z^{\prime}\right)<\beta$ tem-se $\left|l_{0}(z)-l_{0}\left(z^{\prime}\right)\right|<\frac{\varepsilon}{2}$ e usando a convergência dos semigrupos podemos escolher $\eta_{7} \in\left(0, \eta_{6}\right]$ e $\sigma_{4} \in\left(0, \sigma_{3}\right]$ tais que

$$
\sup _{z \in \mathcal{O}_{\sigma_{4}}(x)} \sup _{0 \leq t \leq t_{0}} d\left(T_{\eta}(t) z, T_{0}(t) z\right)<\beta
$$

Logo para todo $z \in \mathcal{O}_{\sigma_{4}}(x)$, todo $t \in\left[0, t_{0}\right]$ e todo $\eta \in\left[0, \eta_{7}\right]$

$$
\begin{gathered}
\left|l_{\eta}\left(T_{\eta}(t) z\right)-l_{0}\left(T_{0}(t) z\right)\right| \leq \\
\left|l_{\eta}\left(T_{\eta}(t) z\right)-l_{0}\left(T_{\eta}(t) z\right)\right|+\left|l_{0}\left(T_{\eta}(t) z\right)-l_{0}\left(T_{0}(t) z\right)\right|<\varepsilon,
\end{gathered}
$$

donde facilmente vemos que

$$
\sup _{z \in \mathcal{O}_{\sigma_{4}}(x)}\left|k_{\eta}(z)-k_{0}(z)\right| \leq \varepsilon \text { sempre que } \eta \in\left[0, \eta_{7}\right]
$$

onde $\sigma_{4}>0$ e $\eta_{7}>0$ dependem apenas de $x \in X$ e de $\varepsilon>0$ dados.

Caso $2: l_{0}(x)=0$. Nestas condições, em primeiro lugar, observamos que $x \in A_{0}$, $\operatorname{logo} k_{0}(x)=0$.

Dado $\varepsilon>0$ escolhamos, pela continuidade de $l_{0}: X \rightarrow \mathbb{R}, \delta>0$ tal que $l_{0}\left(\mathcal{O}_{\delta}\left(A_{0}\right)\right) \subset$ $\left[0, \frac{\varepsilon}{4}\right)$.

Agora, da convergência uniforme em $X$ de $\left(l_{\eta}\right)_{\eta \in[0,1]}$ para $l_{0}$ obtém-se a existência de $\eta_{0} \in(0,1]$ tal que

$$
l_{\eta}\left(\mathcal{O}_{\delta}\left(A_{0}\right)\right) \subset\left[0, \frac{\varepsilon}{2}\right) \text { para cada } \eta \in\left[0, \eta_{0}\right]
$$

Usando a semicontinuidade superior de $\left(A_{\eta}\right)_{\eta \in[0,1]}$ em $\eta=0$ conclui-se a existência de $\eta_{1} \in\left(0, \eta_{0}\right]$ de maneira que para todo $\eta \in\left[0, \eta_{1}\right]$ temos $A_{\eta} \subset \mathcal{O}_{\frac{\delta}{2}}\left(A_{0}\right)$, donde vem que $\mathcal{O}_{\frac{\delta}{2}}\left(A_{\eta}\right) \subset \mathcal{O}_{\delta}\left(A_{0}\right)$ sempre que $\eta \in\left[0, \eta_{1}\right]$ e de (5.2.9) conclui-se que para todo $\eta \in\left[0, \eta_{1}\right]$

$$
l_{\eta}\left(\mathcal{O}_{\frac{\delta}{2}}\left(A_{\eta}\right)\right) \subset\left[0, \frac{\varepsilon}{2}\right) .
$$

Pelo Lema 5.1.2, sejam também $\eta_{2} \in\left(0, \eta_{1}\right]$ e $\delta^{\prime} \in\left(0, \frac{\delta}{2}\right)$ tais que

$$
\gamma_{\eta}^{+}\left(\mathcal{O}_{\delta^{\prime}}\left(A_{\eta}\right)\right) \subset \mathcal{O}_{\frac{\delta}{2}}\left(A_{\eta}\right) \text { para todo } \eta \in\left[0, \eta_{2}\right]
$$


Finalmente, considerando a semicontinuidade inferior de $\left(A_{\eta}\right)_{\eta \in[0,1]}$ em $\eta=0$, seja $\eta_{3} \in\left(0, \eta_{2}\right]$ de maneira que

$$
A_{0} \subset \mathcal{O}_{\frac{\delta^{\prime}}{2}}\left(A_{\eta}\right) \text { para todo } \eta \in\left[0, \eta_{3}\right]
$$

donde conclui-se de (5.2.12) e de (5.2.11) que para todo $\eta \in\left[0, \eta_{3}\right]$, todo $t \geq 0$ e todo $z \in \mathcal{O}_{\frac{\delta^{\prime}}{2}}\left(A_{0}\right) \subset \mathcal{O}_{\delta^{\prime}}\left(A_{\eta}\right)$ tem-se $T_{\eta}(t) z \in \mathcal{O}_{\frac{\delta}{2}}\left(A_{\eta}\right)$ e levando em conta $(5.2 .10)$ deduz-se que para todo $\eta \in\left[0, \eta_{3}\right]$ e todo $z \in \mathcal{O}_{\frac{\delta^{\prime}}{2}}\left(A_{0}\right)$ vale

$$
k_{\eta}(z)=\sup _{t \geq 0} l_{\eta}\left(T_{\eta}(t) z\right) \leq \frac{\varepsilon}{2}
$$

donde vemos, em particular, que

$$
\sup _{z \in \mathcal{O}_{\frac{\delta^{\prime}}{2}}\left(A_{0}\right)}\left|k_{\eta}(z)-k_{0}(z)\right| \leq \varepsilon \text { para todo } \eta \in\left[0, \eta_{3}\right]
$$

com $\delta^{\prime}>0$ e $\eta_{3}>0$ dependendo somente de $\varepsilon>0$ e de $A_{0}$.

Caso $3: T_{0}(t) x \underset{t \rightarrow \infty}{\rightarrow} A_{0}^{*}$. Neste caso temos $k_{0}(x)=1$.

Pela continuidade da função $l_{0}: X \rightarrow \mathbb{R}$, dado $\varepsilon>0$ seja $\delta>0$ tal que

$$
l_{0}\left(\mathcal{O}_{\delta}\left(A_{0}^{*}\right)\right) \subset\left(1-\frac{\varepsilon}{4}, 1\right]
$$

e usando a convergência $l_{\eta} \underset{\eta \rightarrow 0^{+}}{\rightarrow} l_{0}$ uniforme em $X$ escolhamos $\eta_{0} \in(0,1]$ tal que

$$
l_{\eta}\left(\mathcal{O}_{\delta}\left(A_{0}^{*}\right)\right) \subset\left(1-\frac{\varepsilon}{2}, 1\right] \text { para todo } \eta \in\left[0, \eta_{0}\right]
$$

Por outro lado, consideremos $t_{0}>0$ tal que $T_{0}\left(t_{0}\right) x \in \mathcal{O}_{\frac{\delta}{2}}\left(A_{0}^{*}\right)$ e, pela continuidade do operador $T_{0}\left(t_{0}\right): X \rightarrow X$, escolhamos $\sigma_{1}>0$ tal que $T_{0}\left(t_{0}\right)\left(\mathcal{O}_{\sigma_{1}}(x)\right) \subset \mathcal{O}_{\frac{\delta}{2}}\left(A_{0}^{*}\right)$. Usando a hipótese de convergência dos semigrupos, sejam $\eta_{1} \in\left(0, \eta_{0}\right]$ e $\sigma_{2} \in\left(0, \sigma_{1}\right]$ tais que $T_{\eta}\left(t_{0}\right)\left(\mathcal{O}_{\sigma_{2}}(x)\right) \subset \mathcal{O}_{\delta}\left(A_{0}^{*}\right)$ para todo $\eta \in\left[0, \eta_{1}\right]$.

Finalmente, levando em conta (5.2.14), obtém-se $l_{\eta}\left(T_{\eta}\left(t_{0}\right)\left(\mathcal{O}_{\sigma_{2}}(x)\right)\right) \subset\left(1-\frac{\varepsilon}{2}, 1\right]$ para todo $\eta \in\left[0, \eta_{1}\right]$, donde vemos que para todo $z \in \mathcal{O}_{\sigma_{2}}(x)$ e para todo $\eta \in\left[0, \eta_{1}\right]$ tem-se $1-\frac{\varepsilon}{2}<l_{\eta}\left(T_{\eta}\left(t_{0}\right) z\right) \leq k_{\eta}(z) \leq 1$, daí infere-se que $\left|k_{\eta}(z)-k_{0}(z)\right| \leq \varepsilon$ para cada $\eta \in\left[0, \eta_{1}\right]$ e cada $z \in \mathcal{O}_{\sigma_{2}}(x)$, provando que

$$
\sup _{z \in \mathcal{O}_{\sigma_{2}}(x)}\left|k_{\eta}(z)-k_{0}(z)\right| \leq \varepsilon \text { sempre que } \eta \in\left[0, \eta_{1}\right]
$$

onde $\sigma_{2}>0$ e $\eta_{1}>0$ dependem somente de $A_{0}^{*}$ e do $\varepsilon>0$ dado.

Agora, unindo as conclusões obtidas nos casos 1, 2 e 3 podemos concluir a Etapa 2 da seguinte maneira: 
Dados $K \subset X$ um subconjunto compacto de $X$ e $\varepsilon>0$, das desigualdades (5.2.8), (5.2.13) e (5.2.15) e a compacidade de $K$, obtém-se um subconjunto aberto $U=$ $U(\varepsilon, K) \subset X$ com $K \subset U$ e um índice $\eta^{\prime}=\eta^{\prime}(\varepsilon, K)>0$ de maneira que

$$
\sup _{z \in U}\left|k_{\eta}(z)-k_{0}(z)\right| \leq \varepsilon \text { sempre que } \eta \in\left[0, \eta^{\prime}\right]
$$

donde conclui-se facilmente que $\lim _{\eta \rightarrow 0^{+}} \sup _{z \in K}\left|k_{\eta}(z)-k_{0}(z)\right|=0$, completando a prova da Etapa 2.

Etapa 3: $h_{\eta} \underset{\eta \rightarrow 0^{+}}{\rightarrow} h_{0}$ uniformemente em compactos de $X$.

Com efeito, dado $x \in X$, consideremos os seguintes dois casos:

Caso $1: d\left(x, \mathcal{A}_{0}\right)>0$. Neste caso, dado $\alpha>0$ com $0<\alpha<d\left(x, \mathcal{A}_{0}\right)$ sejam, pelo Lema 5.1.2, $\alpha^{\prime} \in(0, \alpha)$ e $\eta_{0} \in(0,1]$ tais que para todo $\eta \in\left[0, \eta_{0}\right]$

$$
\gamma_{\eta}^{+}\left(\mathcal{O}_{\alpha^{\prime}}\left(\mathcal{A}_{\eta}\right)\right) \subset \mathcal{O}_{\alpha}\left(\mathcal{A}_{\eta}\right)
$$

Escolhamos $t_{0}>0$ tal que $T_{0}\left(t_{0}\right) x \in \mathcal{O}_{\frac{\alpha^{\prime}}{4}}\left(\mathcal{A}_{0}\right)$ e pela continuidade do operador $T_{0}\left(t_{0}\right): X \rightarrow X$ seja $\sigma_{1}>0$ tal que $T_{0}\left(t_{0}\right)\left(\mathcal{O}_{\sigma_{1}}^{4}(x)\right) \subset \mathcal{O}_{\frac{\alpha^{\prime}}{4}}\left(\mathcal{A}_{0}\right)$.

Agora, graças à convergência dos semigrupos, sejam $\eta_{1} \in\left(0, \eta_{0}\right]$ e $\sigma_{2} \in\left(0, \sigma_{1}\right]$ tais que $T_{\eta}\left(t_{0}\right)\left(\mathcal{O}_{\sigma_{2}}(x)\right) \subset \mathcal{O}_{\frac{\alpha^{\prime}}{2}}\left(\mathcal{A}_{0}\right)$ para cada $\eta \in\left[0, \eta_{1}\right]$ e pela semicontinuidade inferior da família $\left(\mathcal{A}_{\eta}\right)_{\eta \in[0,1]}$ em $\eta=0$, seja $\eta_{2} \in\left(0, \eta_{1}\right]$ tal que $\mathcal{A}_{0} \subset \mathcal{O}_{\frac{\alpha^{\prime}}{2}}\left(\mathcal{A}_{\eta}\right)$ para todo $\eta \in\left[0, \eta_{2}\right], \log 0 \mathcal{O}_{\frac{\alpha^{\prime}}{2}}\left(\mathcal{A}_{0}\right) \subset \mathcal{O}_{\alpha^{\prime}}\left(\mathcal{A}_{\eta}\right)$ toda vez que $\eta \in\left[0, \eta_{2}\right]$, donde vemos que para todo $\eta \in\left[0, \eta_{2}\right]^{2}$ tem-se $T_{\eta}\left(t_{0}\right)\left(\mathcal{O}_{\sigma_{2}}(x)\right) \subset \mathcal{O}_{\alpha^{\prime}}\left(\mathcal{A}_{\eta}\right)$ e de (5.2.16) obtém-se $\gamma_{\eta}^{+}\left(T_{\eta}\left(t_{0}\right)\left(\mathcal{O}_{\sigma_{2}}(x)\right)\right) \subset \mathcal{O}_{\alpha}\left(\mathcal{A}_{\eta}\right)$ para todo $\eta \in\left[0, \eta_{2}\right]$, donde

$$
\sup _{t \geq t_{0}} d\left(T_{\eta}(t) z, \mathcal{A}_{\eta}\right) \leq \alpha \text { para todo } \eta \in\left[0, \eta_{2}\right] \text { e todo } z \in \mathcal{O}_{\sigma_{2}}(x) .
$$

Por outro lado, é fácil ver que para todo $z \in X$ e todo $\eta \in[0,1]$ temos

$$
\left|d\left(z, \mathcal{A}_{\eta}\right)-d\left(z, \mathcal{A}_{0}\right)\right| \leq d_{H}\left(\mathcal{A}_{\eta}, \mathcal{A}_{0}\right)
$$

então, podemos escolher $\eta_{3} \in\left(0, \eta_{2}\right]$ e $\sigma_{3} \in\left(0, \sigma_{2}\right]$ tais que $d\left(z, \mathcal{A}_{\eta}\right)>\alpha$ para todo $\eta \in\left[0, \eta_{3}\right]$ e todo $z \in \mathcal{O}_{\sigma_{3}}(x)$, donde, levando em conta (5.2.17), obtém-se

$$
\sup _{t \geq t_{0}} d\left(T_{\eta}(t) z, \mathcal{A}_{\eta}\right) \leq \alpha<d\left(z, \mathcal{A}_{\eta}\right) \text { para todo } \eta \in\left[0, \eta_{3}\right] \text { e todo } z \in \mathcal{O}_{\sigma_{3}}(x)
$$

mostrando que $h_{\eta}(z)=\sup _{0 \leq t \leq t_{0}} d\left(T_{\eta}(t) z, \mathcal{A}_{\eta}\right)$ para cada $\eta \in\left[0, \eta_{3}\right]$ e cada $z \in \mathcal{O}_{\sigma_{3}}(x)$.

Observemos agora que para todo $z \in X$, todo $\eta \in[0,1]$ e todo $t \geq 0$ tem-se

$$
\left|d\left(T_{\eta}(t) z, \mathcal{A}_{\eta}\right)-d\left(T_{0}(t) z, \mathcal{A}_{0}\right)\right| \leq d_{H}\left(\mathcal{A}_{\eta}, \mathcal{A}_{0}\right)+d\left(T_{\eta}(t) z, T_{0}(t) z\right),
$$


donde conclui-se, levando em conta o que fizemos acima, que para todo $\eta \in\left[0, \eta_{3}\right]$

$$
\sup _{z \in \mathcal{O}_{\sigma_{3}}(x)}\left|h_{\eta}(z)-h_{0}(z)\right| \leq d_{H}\left(\mathcal{A}_{\eta}, \mathcal{A}_{0}\right)+\sup _{z \in \mathcal{O}_{\sigma_{3}}(x)} \sup _{0 \leq t \leq t_{0}} d\left(T_{\eta}(t) z, T_{0}(t) z\right) .
$$

Assim, é fácil ver que, dado $\varepsilon>0$ existem $\sigma \in\left(0, \sigma_{3}\right]$ e $\eta_{4} \in\left(0, \eta_{3}\right]$ tais que

$$
\sup _{z \in \mathcal{O}_{\sigma}(x)}\left|h_{\eta}(z)-h_{0}(z)\right| \leq \varepsilon, \text { sempre que } \eta \in\left[0, \eta_{4}\right] .
$$

Caso $2: d\left(x, \mathcal{A}_{0}\right)=0$, isto é, $x \in \mathcal{A}_{0}$.

Neste caso, dado $\varepsilon>0$, novamente aplicando o Lema 5.1.2, sejam $\varepsilon^{\prime} \in\left(0, \frac{\varepsilon}{2}\right)$ e $\eta_{0} \in(0,1]$ tais que para todo $\eta \in\left[0, \eta_{0}\right]$

$$
\gamma_{\eta}^{+}\left(\mathcal{O}_{\varepsilon^{\prime}}\left(\mathcal{A}_{\eta}\right)\right) \subset \mathcal{O}_{\frac{\varepsilon}{2}}\left(\mathcal{A}_{\eta}\right)
$$

Por outro lado, usando a semicontinuidade inferior da família $\left(\mathcal{A}_{\eta}\right)_{\eta \in[0,1]}$ em $\eta=0$, seja $\eta_{1} \in\left(0, \eta_{0}\right]$ tal que $\mathcal{A}_{0} \subset \mathcal{O}_{\frac{\varepsilon^{\prime}}{2}}\left(\mathcal{A}_{\eta}\right)$ sempre que $\eta \in\left[0, \eta_{1}\right]$. Portanto, $\mathcal{O}_{\frac{\varepsilon^{\prime}}{2}}\left(\mathcal{A}_{0}\right) \subset$ $\mathcal{O}_{\varepsilon^{\prime}}\left(\mathcal{A}_{\eta}\right)$ sempre que $\eta \in\left[0, \eta_{1}\right]$, e assim (5.2.19) nos diz que

$$
\gamma_{\eta}^{+}\left(\mathcal{O}_{\frac{\varepsilon^{\prime}}{2}}\left(\mathcal{A}_{0}\right)\right) \subset \mathcal{O}_{\frac{\varepsilon}{2}}\left(\mathcal{A}_{\eta}\right), \text { toda vez que } \eta \in\left[0, \eta_{1}\right]
$$

donde segue-se que $h_{\eta}(z)=\sup _{t \geq 0} d\left(T_{\eta}(t) z, \mathcal{A}_{\eta}\right) \leq \frac{\varepsilon}{2}$, sempre que $\eta \in\left[0, \eta_{1}\right]$ e $z \in$ $\mathcal{O}_{\frac{\varepsilon^{\prime}}{2}}\left(\mathcal{A}_{0}\right)$, concluindo-se que

$$
\sup _{z \in \mathcal{O}_{\frac{\varepsilon^{\prime}}{2}}\left(\mathcal{A}_{0}\right)}\left|h_{\eta}(z)-h_{0}(z)\right| \leq \varepsilon, \text { sempre que } \eta \in\left[0, \eta_{1}\right]
$$

Vemos assim que, dado $\varepsilon>0$, cada ponto $x$ de $X$ possui uma vizinhança $\mathcal{O}_{\sigma}(x)$, com $\sigma=\sigma(\varepsilon, x)>0$ e associado a esta vizinhança existe um índice $\eta^{\prime}=\eta^{\prime}(\varepsilon, x)>0$ de modo que

$$
\sup _{z \in \mathcal{O}_{\sigma}(x)}\left|h_{\eta}(z)-h_{0}(z)\right| \leq \varepsilon, \text { toda vez que } \eta \in\left[0, \eta^{\prime}\right]
$$

donde conclui-se a convergência $h_{\eta} \underset{\eta \rightarrow 0^{+}}{\rightarrow} h_{0}$, uniforme sobre compactos de $X$, da mesma maneira que fizemos no final da Etapa 2, completando a demonstração da proposição.

Observação 5.2.2. Seja $\left\{T_{\eta}(t): t \geq 0\right\}_{\eta \in[0,1]}$ uma família de semigrupos em um espaço métrico $X:=(X, d)$ satisfazendo as hipóteses do Teorema 3.2 .4 (de Carvalho e Langa) com família finita disjunta de conjuntos invariantes isolados dada por $\Xi_{\eta}:=\left\{\Xi_{1, \eta}, \cdots, \Xi_{n, \eta}\right\}$. 
Conforme foi estabelecido naquele Teorema, para cada $\eta$ suficientemente pequeno $\left\{T_{\eta}(t): t \geq 0\right\}$ é um semigrupo de tipo gradiente generalizado com respeito à família $\boldsymbol{\Xi}_{\eta}$ e, pelo Teorema 4.3.3, cada família $\boldsymbol{\Xi}_{\eta}$ determina (após uma possível reordenação que, a priori, pode depender de $\eta$ ) uma decomposição de Morse para o atrator $\mathcal{A}_{\eta}$.

Seja $\sigma: I_{n} \rightarrow I_{n}$ uma permutação (fixada) tal que, segundo o Teorema 4.3.3, a $n$-upla ordenada $\mathbf{D}_{0}:=\left(\Xi_{\sigma(1), 0}, \Xi_{\sigma(2), 0}, \cdots, \Xi_{\sigma(n), 0}\right)$ é uma decomposição de Morse para o atrator $\mathcal{A}_{0}$ do semigrupo limite $T_{0}(\cdot)$ e consideremos, para as demais famílias $\boldsymbol{\Xi}_{\eta}$, a mesma reordenação determinada por $\sigma: I_{n} \rightarrow I_{n}$, definindo, para $\eta \in(0,1]$, a $n$-upla ordenada $\mathbf{D}_{\eta}:=\left(\Xi_{\sigma(1), \eta}, \Xi_{\sigma(2), \eta}, \cdots, \Xi_{\sigma(n), \eta}\right)$.

Consideremos agora, imitando a construção em (4.3.6), para $\eta \in(0,1]$ o conjunto $A_{0, \eta}:=\varnothing$ e para cada $j=1,2, \cdots, n$

$$
A_{j, \eta}:=\bigcup_{i=1}^{j} W_{\eta}^{u}\left(\Xi_{\sigma(i), \eta}\right) .
$$

Então, para $\eta>0$ suficientemente pequeno, $A_{j, \eta}$ é, para todo $j=1,2, \cdots, n$, um atrator local para $T_{\eta}(\cdot)$ com

$$
\Xi_{\sigma(j), \eta}=A_{j, \eta} \cap A_{j-1, \eta}^{*}
$$

para todo $j=1,2, \cdots, n^{1}$.

Em outras palavras, a reordenação da família $\Xi_{0}$, definida por $\sigma: I_{n} \rightarrow I_{n}$, determinando a decomposição de Morse $\mathbf{D}_{0}=\left(\Xi_{\sigma(1), 0}, \Xi_{\sigma(2), 0}, \cdots, \Xi_{\sigma(n), 0}\right)$ do atrator $\mathcal{A}_{0}$ também determina, para $\eta>0$ suficientemente pequeno, uma decomposição de Morse $\mathbf{D}_{\eta}=\left(\Xi_{\sigma(1), \eta}, \Xi_{\sigma(2), \eta}, \cdots, \Xi_{\sigma(n), \eta}\right)$ para o atrator $\mathcal{A}_{\eta}$ com conjuntos de Morse sendo, precisamente, os elementos da família $\boldsymbol{\Xi}_{\eta}$. Em particular, esta decomposição de Morse se comporta continuamente.

Além disso, para $\eta$ suficientemente pequeno, aplicando diretamente a definição de repulsor complementar, vemos facilmente que a cadeia correspondente de repulsores vem dada por:

$$
\begin{aligned}
& A_{n, \eta}^{*}:=\varnothing \text { e para cada } j=0,1, \cdots, n-1 \\
& A_{j, \eta}^{*}:=\bigcup_{i=j+1}^{n} W_{\eta}^{s}\left(\Xi_{\sigma(i), \eta}\right),
\end{aligned}
$$

onde aqui, estes conjuntos estáveis estão sendo considerados restritos a seus atratores globais correspondentes, ou seja, para todo $\eta \in[0,1]$ e todo $j=1,2, \cdots, n$

$$
W_{\eta}^{s}\left(\Xi_{j, \eta}\right):=\left\{x \in \mathcal{A}_{\eta}: \lim _{t \rightarrow \infty} d\left(T_{\eta}(t) x, \Xi_{j, \eta}\right)=0\right\} .
$$

\footnotetext{
${ }^{1}$ Veja a Seção 7.5 para uma demonstração destes fatos no contexto mais geral de perturbações não autônomas de semigrupos gradientes.
} 
Sendo assim, qualquer condição suficiente para que os conjuntos estáveis (restritos aos atratores globais) e instáveis se comportem continuamente será também, segundo o que vimos na Proposição 5.2.1 em conjunto com o Teorema 4.4.1, uma condição suficiente para garantir a convergência uniforme em compactos das funções de Lyapunov dos semigrupos de tipo gradiente. 


\section{Níveis de energia de um semigrupo gradiente}

Neste último capítulo da teoria autônoma construímos uma nova decomposição de Morse para o atrator de um semigrupo gradiente, que nos proporciona novas informações sobre o comportamento assintótico das soluções do sistema, no sentido de classificar invariantes isolados que possuem características dinâmicas semelhantes, estabelecendo uma ordem ou um sentido para o deslocamento das soluções. Tal decomposição está baseada no conceito que batizamos como "níveis de energia", os quais, ainda que se possam definir para conjuntos invariantes isolados quaisquer, simplesmente trocando as notações, restringiremos nossa análise ao contexto dos conjuntos finitos de pontos de equílibrio.

Deixemos registrado, contudo, que os conteúdos discutidos neste capítulo podem ser omitidos pelo leitor, não trazendo prejuizo algum ao entendimento do restante do texto.

\subsection{Estrutura dos níveis}

Definição 6.1.1. Seja $\{T(t): t \geq 0\}$ um semigrupo em um espaço métrico $X$ possuindo atrator global $\mathcal{A}$ e um número finito de pontos de equilíbrio $\mathcal{E}:=\left\{z_{1}^{*}, \cdots, z_{p}^{*}\right\}$. Definimos:

(1) Dados $z^{*}$ e $w^{*}$ em $\mathcal{E}$ escreve-se $z^{*} \succ w^{*}$, e neste caso diz-se que $z^{*}$ segue $w^{*}$ ou ainda que $w^{*}$ precede $z^{*}$, quando existe uma solução global nãa constante 
$\xi: \mathbb{R} \rightarrow X$ para $\{T(t): t \geq 0\}$ satisfazendo

$$
\lim _{t \rightarrow-\infty} \xi(t)=z^{*} e \lim _{t \rightarrow \infty} \xi(t)=w^{*}
$$

(2) Os conjuntos:

$$
\begin{aligned}
& \mathcal{M}_{1}:=\left\{z^{*} \in \mathcal{E}: \text { para todo } w^{*} \in \mathcal{E}, \text { se } z^{*} \succ w^{*} \text { então } w^{*}=z^{*}\right\} \\
& \mathcal{M}_{2}:=\left\{z^{*} \in \mathcal{E}: \text { para todo } w^{*} \in \mathcal{E}, \text { se } z^{*} \succ w^{*} \text { então } w^{*} \in \mathcal{M}_{1}\right\}
\end{aligned}
$$

Analogamente para todo natural $k$

$$
\mathcal{M}_{k+1}:=\left\{z^{*} \in \mathcal{E}: \text { para todo } w^{*} \in \mathcal{E}, \text { se } z^{*} \succ w^{*} \text { então } w^{*} \in \mathcal{M}_{k}\right\}
$$

Observemos que $\mathcal{M}_{k} \subset \mathcal{M}_{k+1}$ qualquer que seja $k \in \mathbb{N}$.

(3) Nas condições de (2) acima, para cada natural $k$, o k-ésimo nível de energia de $\{T(t): t \geq 0\}$ é o conjunto $\mathcal{N}_{k}$ dado por

$$
\begin{gathered}
\mathcal{N}_{1}:=\mathcal{M}_{1} e \\
\mathcal{N}_{k}:=\mathcal{M}_{k} \backslash \mathcal{M}_{k-1} \text { quando } k \geq 2 .
\end{gathered}
$$

Devemos observar que, nas condições acima, existe um natural $q$ tal que $\mathcal{N}_{k}=\varnothing$ sempre que $k>q$, em outras palavras, $\mathcal{M}_{k}=\mathcal{M}_{q}$ sempre que $k>q$. Pois, caso contrário, estaríamos com uma quantidade infinita de pontos de equilíbrio. Desta forma, de agora em diante, consideramos como níveis de energía, somente os conjuntos (potencialmente não triviais) $\mathcal{N}_{1}, \cdots, \mathcal{N}_{n}$, onde

$$
n:=\min \left\{q \in \mathbb{N}: \mathcal{M}_{k}=\mathcal{M}_{q} \text { para todo } k>q\right\},
$$

os quais serão fixados, de uma vez por todas, para referências futuras ao longo do capítulo.

Notemos que, para um semigrupo arbitrário, é possível que um certo ponto de equilíbrio $z^{*}$ não pertença a nenhum dos níveis de energia como acima definidos. Este é o caso quando, por exemplo, o ponto $z^{*}$ figura em uma estrutura homoclínica que possui mais do que um vértice.

Finalmente, observemos que se $z^{*}$ e $w^{*}$ são pontos de equilíbrio distintos em um mesmo nível $\mathcal{N}_{i}$, então não pode ocorrer $z^{*} \succ w^{*}$, dado que para $i \geq 2$, como $z^{*} \in \mathcal{M}_{i}$, a condição $z^{*} \succ w^{*}$ implicaria $w^{*} \in \mathcal{M}_{i-1}$, contradizendo $\mathcal{N}_{i}=\mathcal{M}_{i} \backslash \mathcal{M}_{i-1}$. O caso $i=1$ é evidente. 
Das observações anteriores, surge a questão de tentar determinar quais semigrupos têm a propriedade de possuir seu conjunto de pontos de equilíbrio escrito como a reunião de seus níveis de energia. Do que acabamos de ver acima, uma condição necessária para que um semigrupo tenha esta propriedade, é que seu atrator não possua estruturas homoclínicas. Então, é natural pensar que ela se verifica para os semigrupos de tipo gradiente e, de fato, estes semigrupos têm seus níveis de energia bem definidos, como nos mostra o seguinte resultado:

Lema 6.1.2. Se $\{T(t): t \geq 0\}$ é um semigrupo de tipo gradiente, então todo $z^{*} \in \mathcal{E}$ está em $\mathcal{N}_{i}$ para algum $i=1, \cdots, n$, onde $n \geq 1$ é o número máximo de níveis de energia não vazios de $\{T(t): t \geq 0\}$.

Demonstração: Com efeito, suponhamos primeiramente que $\mathcal{N}_{1}$ seja vazio e fixemos $z_{l_{1}}^{*} \in \mathcal{E}$ qualquer. Então, como $z_{l_{1}}^{*} \notin \mathcal{N}_{1}$, pela definição de $\mathcal{N}_{1}$, existe $z_{l_{2}}^{*} \in \mathcal{E}$ com $z_{l_{1}}^{*} \succ z_{l_{2}}^{*}$ e $z_{l_{2}}^{*} \neq z_{l_{1}}^{*}$. Analogamente, $z_{l_{2}}^{*} \notin \mathcal{N}_{1}$, donde existe $z_{l_{3}}^{*} \in \mathcal{E}$ com $z_{l_{2}}^{*} \succ z_{l_{3}}^{*}$ e $z_{l_{3}}^{*} \neq z_{l_{2}}^{*}$. Também $z_{l_{3}}^{*} \neq z_{l_{1}}^{*}$, porque, de outro modo, teríamos a estrutura homoclínica $z_{l_{1}}^{*} \succ z_{l_{2}}^{*} \succ z_{l_{1}}^{*}$, contradizendo a propriedade $(G 2)$. Prosseguindo analogamente, levando em conta que $\mathcal{E}$ é um conjunto finito, este procedimento deverá parar em um número finito de passos. Assim, na etapa final chegaremos à conclusão de que, ou o ponto de equilíbrio $z_{l_{k}}^{*}$, que lá aparecer, formará uma estrutura homoclínica com alguns dos pontos $z_{l_{i}}^{*}$ 's que apareceram nos passos anteriores, ou este ponto está em $\mathcal{N}_{1}$, as quais são duas situações contraditórias e, portanto, tem-se $\mathcal{N}_{1} \neq \varnothing$.

Se $\mathcal{E}=\mathcal{N}_{1}$, então acabamos. Caso contrário, tem-se também $\mathcal{N}_{2}$ não vazio. De fato, se $\mathcal{N}_{2}$ é vazio, fixado $z_{l_{1}}^{*} \in \mathcal{E} \backslash \mathcal{N}_{1}, z_{l_{1}}^{*}$ não pertence a $\mathcal{N}_{2}$, portanto existe $z_{l_{2}}^{*} \in \mathcal{E} \backslash \mathcal{N}_{1}$ com $z_{l_{1}}^{*} \succ z_{l_{2}}^{*}$ e, por $(G 2), z_{l_{2}}^{*} \neq z_{l_{1}}^{*}$. Da mesma forma, também $z_{l_{2}}^{*} \notin \mathcal{N}_{2}$, consequentemente existe $z_{l_{3}}^{*} \in \mathcal{E} \backslash \mathcal{N}_{1}$ com $z_{l_{2}}^{*} \succ z_{l_{3}}^{*}$ e, além disso, por $(G 2)$ temos $z_{l_{3}}^{*} \neq z_{l_{2}}^{*}$ e $z_{l_{3}}^{*} \neq z_{l_{1}}^{*}$. Continuando com o raciocínio chegaremos a uma contradição análoga à que ocorreu na prova da primeira parte acima, donde segue-se que $\mathcal{N}_{2} \neq \varnothing$.

Caso $\mathcal{E}=\mathcal{N}_{1} \cup \mathcal{N}_{2}=\mathcal{M}_{2}$, acabou. Se isto não ocorre, então também $\mathcal{N}_{3} \neq \varnothing$. De fato, tomando $z_{l_{1}}^{*} \in \mathcal{E} \backslash\left(\mathcal{N}_{1} \cup \mathcal{N}_{2}\right)=\mathcal{E} \backslash \mathcal{M}_{2}$ arbitrário, podemos, de forma semelhante, repetir o argumento precedente, daí seremos novamente levados a uma contradição e, portanto, a concluir que $\mathcal{N}_{3} \neq \varnothing$.

Continuando com a idéia, a mesma deverá terminar após o número finito $n \in$ $\{1, \cdots, p\}$ de passos, onde teremos estudado todas as possibilidades para os elementos de $\mathcal{E}$, completando a demonstração.

Destaquemos agora algumas das propriedades que possuem os níveis de energia de um semigrupo gradiente. 
Observemos, em primeiro lugar que, na demonstração do Lema 6.1.2, em particular, ficou provado que para um semigrupo de tipo gradiente seu nível de energia $\mathcal{N}_{1}$ é sempre não vazio e, de fato, ele está formado por aqueles pontos de equilíbrio que não possuem soluções globais emanando deles, mais precisamente temos:

Definição 6.1.3. Um ponto de equilíbrio $z^{*}$ de um semigrupo $\{T(t): t \geq 0\}$ chamase assintoticamente estável, quando é possível encontrar $\varepsilon>0$ tal que para todo $x \in X \operatorname{com} d\left(x, z^{*}\right)<\varepsilon$ tem-se $T(t) x \rightarrow z^{*}$ quando $t \rightarrow \infty$.

Lema 6.1.4. Se $\{T(t): t \geq 0\}$ é um semigrupo gradiente, então todos os seus pontos de equilíbrio em $\mathcal{N}_{1}$ são assintoticamente estáveis. Além disso, se n é como no Lema 6.1.2 e é maior do que ou igual a 2 , dado $2 \leq i \leq n$, para cada $z^{*} \in \mathcal{N}_{i}$ existe $\delta=\delta\left(z^{*}\right)>0$ tal que se $d\left(x, z^{*}\right)<\delta$ então, ou

$$
d\left(T(t) x, z^{*}\right) \underset{t \rightarrow \infty}{\rightarrow} 0
$$

ou

$$
d\left(T(t) x, \mathcal{M}_{i-1}\right) \underset{t \rightarrow \infty}{\rightarrow} 0 .
$$

Demonstração: Com efeito, seja $z^{*} \in \mathcal{N}_{1}$ um equilíbrio que não seja assintoticamente estável, então para cada natural $k$ podemos encontrar $x_{k} \in X \operatorname{com} d\left(x_{k}, z^{*}\right)<\frac{1}{k}$ de maneira que $T(t) x_{k}$ não converge para $z^{*}$ quando $t \rightarrow \infty$. Assim, sendo $\{T(t): t \geq 0\}$ um semigrupo gradiente, para cada $k$ tem-se

$$
\lim _{t \rightarrow \infty} d\left(T(t) x_{k}, \mathcal{E} \backslash\left\{z^{*}\right\}\right)=0,
$$

Então, para cada $k$ podemos encontrar $t_{k}>0$ tal que $d\left(T(t) x_{k}, z^{*}\right)<\delta$ para $0 \leq t<t_{k}$ e $d\left(T\left(t_{k}\right) x_{k}, z^{*}\right)=\delta$, onde $\delta>0$ é tal que as $\delta$-vizinhanças dos pontos de $\mathcal{E}$ são disjuntos entre si. Nestas condições temos $t_{k} \rightarrow \infty$, pois do contrário, podemos supor $t_{k} \rightarrow t_{0}$, o que nos leva à contradição $d\left(z^{*}, z^{*}\right)=d\left(T\left(t_{0}\right) z^{*}, z^{*}\right)=\delta$. Assim, definindo $\xi_{k}:\left[-t_{k}, \infty\right) \rightarrow X$ por $\xi_{k}(t)=T\left(t+t_{k}\right) x_{k}, t \in\left[-t_{k}, \infty\right)$, do Lema 3.2.3 segue-se que $\left(\xi_{k}\right)_{k \in \mathbb{N}}$ possui uma subsequência, que indicamos com a mesma notação, convergindo uniformemente sobre compactos da reta para uma solução global $\xi: \mathbb{R} \rightarrow$ $X$ de $\{T(t): t \geq 0\}$.

Como $\xi_{k}(t) \in \mathcal{O}_{\delta}\left(z^{*}\right)$ para todo $t \leq 0$ e todo $k \geq k(t)$, para um certo $k(t) \in \mathbb{N}$, segue-se também que $\xi(t) \in \mathcal{O}_{\delta}\left(z^{*}\right)$ para todo $t \leq 0$, e sendo $\{T(t): t \geq 0\}$ de tipo gradiente resulta que $\xi(t) \underset{t \rightarrow-\infty}{\rightarrow} z^{*}$. Por outro lado, $\xi$ é solução não constante porque $\xi(0)=\lim _{k \rightarrow \infty} T\left(t_{k}\right) x_{k}=x \operatorname{com} d\left(z^{*}, x\right)=\delta$. Como $z^{*} \in \mathcal{N}_{1}$, tem-se $\xi(t) \underset{t \rightarrow \infty}{\rightarrow} z^{*}$, portanto $\xi$ é uma solução homoclínica para $\{T(t): t \geq 0\}$, o que contradiz $(G 2)$. Logo, todo ponto de equilíbrio em $\mathcal{N}_{1}$ é assintoticamente estável. 
Agora, suponhamos que $n$ seja maior do que 1 no Lema 6.1 .2 e que $z^{*} \in \mathcal{N}_{2}$ é tal que nossa afirmação não se verifica, então para cada natural $k$ podemos encontrar $x_{k} \in X$ com $d\left(x_{k}, z^{*}\right)<\frac{1}{k}$ tal que $T(t) x_{k}$ não converge para $z^{*}$ quando $t \rightarrow \infty$ e além disso

$$
d\left(T(t) x_{k}, \mathcal{N}_{1}\right) \geq \varepsilon \text { qualquer que seja } t \geq 0,
$$

onde $\varepsilon>0$ é o da estabilidade assintótica comum a todos os elementos de $\mathcal{N}_{1}$ obtida no caso anterior. Analogamente ao que fizemos acima e com a mesma justificativa, para cada $k$ seja $t_{k}>0$ tal que $d\left(T(t) x_{k}, z^{*}\right)<\delta$ para $0 \leq t<t_{k}$ e $d\left(T\left(t_{k}\right) x_{k}, z^{*}\right)=\delta$ qualquer que seja $k$, onde $\delta>0$ é como antes, mas agora satisfazendo também $\delta<$ $\varepsilon$. Portanto $t_{k} \rightarrow \infty$. Definindo $\xi_{k}:\left[-t_{k}, \infty\right) \rightarrow X$ por $\xi_{k}(t)=T\left(t+t_{k}\right) x_{k}, t \in$ $\left[-t_{k}, \infty\right)$. Resulta que $\left(\xi_{k}\right)_{k \in \mathbb{N}}$ possui subsequência, que indicamos da mesma maneira, convergindo uniformemente em compactos da reta a uma solução global $\xi: \mathbb{R} \rightarrow X$ de $\{T(t): t \geq 0\}$. Como $\xi_{k}(t) \in \mathcal{O}_{\delta}\left(z^{*}\right)$ para todo $t \leq 0$ e todo $k$ suficientemente grande, segue-se também que $\xi(t) \in \mathcal{O}_{\delta}\left(z^{*}\right)$ para todo $t \leq 0$, e sendo $\{T(t): t \geq 0\}$ um semigrupo gradiente, resulta que $\xi(t) \underset{t \rightarrow-\infty}{\rightarrow} z^{*}$. Também, $\xi$ é não constante e como $z^{*} \in \mathcal{N}_{2}$, por definição, existe $\tau>0$ de modo que $\xi(t) \in \mathcal{O}_{\delta}\left(\mathcal{N}_{1}\right)$ sempre que $t \geq \tau$. E é fácil ver que este fato contradiz (6.1.1).

Da mesma maneira provam-se as afirmações correspondentes aos casos $3 \leq i \leq n$, recordando que, na prova de cada caso toma-se o menor dos raios das vizinhanças dos níveis obtidos nas etapas anteriores satisfazendo as correspondentes propriedades e o lema fica assim demonstrado.

O lema precedente pode ser usado para provar o resultado abaixo, o qual é, simplesmente, outra maneira de interpretá-lo.

Lema 6.1.5. Sejam $\{T(t): t \geq 0\}$ um semigrupo de tipo gradiente num espaço métrico $X$ e $x \in X$ um ponto qualquer. Se $T(t) x \rightarrow z^{*}$ quando $t \rightarrow \infty e z^{*} \in \mathcal{N}_{i}$ para algum $i=1, \cdots, n$, então existe $\delta=\delta(x)>0$ tal que, para todo $z \in X \operatorname{com} d(z, x)<\delta$ tem-se

$$
d\left(T(t) z, \mathcal{M}_{i}\right) \underset{t \rightarrow \infty}{\rightarrow} 0 .
$$

Demonstração: Usando o lema anterior, para cada $k=1, \cdots, n$, encontra-se facilmente um $\varepsilon=\varepsilon(k)>0$ com a seguinte propriedade:

Se $w^{*} \in \mathcal{M}_{k}$ e $d\left(w, w^{*}\right)<\varepsilon$ então $d\left(T(t) w, \mathcal{M}_{k}\right) \rightarrow 0$ quando $t \rightarrow \infty$.

Portanto, se a conclusão do lema não é correta, existe uma sequência $\left(x_{j}\right)_{j \in \mathbb{N}}$ em $X$ $\operatorname{com} x_{j} \underset{j \rightarrow \infty}{\rightarrow} x$ tal que

$$
d\left(T(t) x_{j}, \mathcal{M}_{i}\right) \geq \varepsilon \text { quaisquer que sejam } t \geq 0 \text { e } j \in \mathbb{N}
$$


onde o $\varepsilon>0$ depende de $k$ como acima.

Assim, fixando $t \geq 0$ em (6.1.2) e fazendo $j \rightarrow \infty$ obtém-se

$$
d\left(T(t) x, \mathcal{M}_{i}\right) \geq \varepsilon \text { qualquer que seja } t \geq 0,
$$

o que evidentemente contradiz $T(t) x \rightarrow z^{*}$ quando $t \rightarrow \infty$ porque $z^{*} \in \mathcal{N}_{i}$.

Observação 6.1.6. Observemos que, se $n$ é como no Lema 6.1.2, então para todo $z^{*} \in \mathcal{N}_{n}$ não pode existir solução global limitada não constante $\xi: \mathbb{R} \rightarrow X$ para $\{T(t): t \geq 0\}$ com $\lim _{t \rightarrow \infty} \xi(t)=z^{*}$.

Com efeito, isto é óbvio para o caso em que $n=1$, se for $n \geq 2$ e $\xi$ é uma tal solução, sendo $\{T(t): t \geq 0\}$ um semigrupo gradiente existe $w^{*} \in \mathcal{E}$ tal que $\lim _{t \rightarrow-\infty} \xi(t)=w^{*}$. Do Lema $6.1 .2, w^{*} \in \mathcal{N}_{i}$ para algum $1 \leq i \leq n$ e da definição dos níveis de equilíbrio deve-se ter $z^{*} \in \mathcal{M}_{i-1}$, o que contradiz $z^{*} \in \mathcal{N}_{n}$.

Mostremos agora que os níveis, como definimos, também constituem uma decomposição de Morse para o atrator de um semigrupo de tipo gradiente.

Teorema 6.1.7. Sejam $\{T(t): t \geq 0\}$ um semigrupo de tipo gradiente com respeito ao conjunto finito de pontos estacionários

$$
\mathcal{E}=\left\{z_{1}^{*}, z_{2}^{*}, \cdots, z_{p}^{*}\right\}
$$

e $\mathcal{A}$ seu atrator global. Se

$$
\mathcal{N}:=\left\{\mathcal{N}_{1}, \mathcal{N}_{2}, \cdots, \mathcal{N}_{n}\right\}
$$

é a família formada pelos níveis de energia de $T(\cdot)$, então $\mathcal{N}$ determina uma decomposição de Morse para o atrator $\mathcal{A}$ de $T(\cdot)$.

Demonstração: Utilizaremos o Teorema 4.3.3.

Para isso, em primeiro lugar, observemos que, como $T(\cdot)$ não possui soluções homoclínicas associadas a $\mathcal{E}$ e não pode haver conexão entre pontos distintos de um mesmo nível, cada nível $\mathcal{N}_{j}$ é um conjunto invariante isolado e, claramente, os elementos de $\mathcal{N}$ formam uma família finita disjunta, logo os elementos de $\mathcal{N}$ formam uma família finita disjunta de conjuntos invariantes isolados. Provemos $T(\cdot)$ é também de tipo gradiente com respeito a ela.

Com efeito, como $T(\cdot)$ é de tipo gradiente com respeito a $\mathcal{E}$, o par $(T(\cdot), \mathcal{N})$ herda a propriedade $(G 1)$ da Definição 3.1.1. Para ver que também satisfaz $(G 2)$, sejam $\mathcal{N}_{l_{1}}, \mathcal{N}_{l_{2}}, \cdots, \mathcal{N}_{l_{k}}$ elementos de $\mathcal{N}$ e $\left\{\xi_{j}: \mathbb{R} \rightarrow X: 1 \leq j \leq k\right\}$ soluções globais não triviais formando uma estrutura homoclínica associadas a $\mathcal{N}$. Das propriedades 
do semigrupo de tipo gradiente $(T(\cdot), \mathcal{E})$ segue-se que, para cada $j=1, \cdots, n$ existe $z_{l_{j}}^{*} \in \mathcal{N}_{j}$ de maneira que

$$
\xi_{j}(t) \underset{t \rightarrow-\infty}{\rightarrow} z_{l_{j}}^{*} \text { e } \xi_{j}(t) \underset{t \rightarrow \infty}{\rightarrow} z_{l_{(j+1)}^{*}}^{*}
$$

o que nos dá uma contradição pois, pela definição de estrutura homoclínica, $\mathcal{N}_{l_{(k+1)}}:=$ $\mathcal{N}_{l_{1}}$ e daí segue-se que $z_{l_{1}}^{*}$ e $z_{l_{(k+1)}}^{*}$ pertencem a $\mathcal{N}_{l_{1}}$ o que contradiz o fato de que o nível $\mathcal{N}_{l_{k}}$ deveria ser mais baixo do que o nível $\mathcal{N}_{l_{1}}$, de acordo com a definição dos níveis, completando a prova.

Segue, do teorema anterior, que toda a teoria que desenvolvemos no Capítulo 4, para famílias arbitrárias de conjuntos invariantes isolados, pode ser aplicada no caso particular em que a família de conjuntos invariantes isolados vem dada pela família dos níveis de energia. Resumindo, tem-se o seguinte resultado:

Corolário 6.1.8. Sejam $\{T(t): t \geq 0\}$ um semigrupo de tipo gradiente com respeito ao conjunto finito de pontos estacionários

$$
\mathcal{E}=\left\{z_{1}^{*}, z_{2}^{*}, \cdots, z_{p}^{*}\right\}
$$

e $\mathcal{A}$ seu atrator global.

Se $\mathcal{N}:=\left\{\mathcal{N}_{1}, \mathcal{N}_{2}, \cdots, \mathcal{N}_{n}\right\}$ é a família formada pelos níveis de energia de $T(\cdot)$, então $(T(\cdot), \mathcal{N})$ é um semigrupo gradiente possuindo função de Lyapunov $V: X \rightarrow \mathbb{R}$ diferenciável ao longo de soluções de $T(\cdot)$ e que, além disso, satisfaz $V\left(z^{*}\right)=k$ sempre que $z^{*} \in \mathcal{N}_{k}$, para todo $k=1, \cdots, n$.

Demonstração: Levando em conta tudo o que fizemos até o momento, o único ponto que devemos demonstrar é que a $n$-upla ordenada $\mathbf{D}:=\left(\mathcal{N}_{1}, \mathcal{N}_{2}, \cdots, \mathcal{N}_{n}\right)$ é uma decomposição de Morse para o atrator $\mathcal{A}$.

Com efeito, como todos os pontos em $\mathcal{N}_{1}$ são assintoticamente estáveis, temos $W^{u}\left(\mathcal{N}_{1}\right)=\mathcal{N}_{1}$ o que nos dá, graças à Observação 4.1.3, que para todo $\delta>0$ existe $\delta^{\prime} \in(0, \delta)$ tal que

$$
\gamma^{+}\left(\mathcal{O}_{\delta^{\prime}}\left(\mathcal{N}_{1}\right)\right) \subset \mathcal{O}_{\delta}\left(\mathcal{N}_{1}\right)
$$

Como $\mathcal{N}_{1}$ é um invariante isolado, resulta que $\omega\left(\mathcal{O}_{\delta}\left(\mathcal{N}_{1}\right)\right)=\mathcal{N}_{1}$, mostrando que $\mathcal{N}_{1}$ é um atrator local.

Agora, é fácil ver que $W^{u}\left(\mathcal{N}_{2}\right) \cap \mathcal{N}_{1}^{*}=\mathcal{N}_{2}$. Portanto, para todo $\delta>0$ existe $\delta^{\prime} \in(0, \delta)$ tal que

$$
\gamma^{+}\left(\mathcal{O}_{\delta^{\prime}}\left(\mathcal{N}_{2}\right)\right) \cap \mathcal{N}_{1}^{*} \subset \mathcal{O}_{\delta}\left(\mathcal{N}_{2}\right) \cap \mathcal{N}_{1}^{*}
$$

Daí resulta que $\omega\left(\mathcal{O}_{\delta}\left(\mathcal{N}_{2}\right) \cap \mathcal{N}_{1}^{*}\right)=\mathcal{N}_{2}$, dizendo que $\mathcal{N}_{2}$ é um atrator local para a restrição do semigrupo $T(\cdot)$ ao invariante $\mathcal{N}_{1}^{*}$ (repulsor complementar do atrator local $\left.\mathcal{N}_{1}\right)$. 
Proseguindo analogamente até $n$, a conclusão do corolário seguirá do Teorema 4.3.3 e da construção que o antecede, terminando a demonstração.

\subsection{Perturbação de níveis}

No que segue, estudamos o comportamento da estrutura dos níveis de energia de um semigrupo gradiente com respeito a perturbações autônomas pequenas. Para isso, usamos algumas das técnicas desenvolvidas em [8], sob certas condições adicionais bastante razoáveis. Mais precisamente, sabemos do Capítulo 3 que o conceito de semigrupo de tipo gradiente é estável por perturbação, donde segue-se que os semigrupos perturbados continuam, pelo Lema 6.1.2, possuindo conjunto de equilíbrios escrito como a reunião de seus níveis de energia. Nestas condições, provaremos que, por exemplo, quando as conexões entre os pontos de equilíbrio do semigrupo limite são mantidas por perturbação, os níveis de energia dos semigrupos perturbados se mantêm em número e, além disso, se comportam continuamente.

O seguinte lema nos mostra a estrutura que têm os pontos de equilíbrio dos semigrupos perturbados. Ele constitui uma extensão do Lema 6.1.4 para o caso de famílias de semigrupos de tipo gradiente e é útil na prova do resultado sobre perturbação de níveis.

Lema 6.2.1. Suponhamos que a família $\left\{T_{\eta}(t): t \geq 0\right\} \eta \in[0,1]$ de semigrupos de tipo gradiente esteja nas condições do Teorema 3.2.4.

Sejam $z_{0}^{*} \in \mathcal{N}_{1,0} e\left(z_{\eta}^{*}\right)_{\eta \in[0,1]}$ a única família com $z_{\eta}^{*} \in \mathcal{E}_{\eta}$, para todo $\eta \in[0,1]$, tal que $z_{\eta}^{*} \underset{\eta \rightarrow 0^{+}}{\rightarrow} z_{0}^{*}$, então existem $\delta>0$ e $\eta_{1} \in(0,1]$ de modo que, qualquer que seja $\eta \in\left[0, \eta_{1}\right]$, se $x \in X$ é tal que $d\left(x, z_{\eta}^{*}\right)<\delta$ então $T_{\eta}(t) x \rightarrow z_{\eta}^{*}$ quando $t \rightarrow \infty$.

Além disso, para $i \geq 2$ se $z_{0}^{*} \in \mathcal{N}_{i, 0}$ e $\left(z_{\eta}^{*}\right)_{\eta \in[0,1]}$ é a única família com $z_{\eta}^{*} \in \mathcal{E}_{\eta}$ tal que $z_{\eta}^{*} \underset{\eta \rightarrow 0^{+}}{\rightarrow} z_{0}^{*}$, então existem $\delta>0$ e $\eta_{i} \in(0,1]$ de maneira que para qualquer $\eta \in\left[0, \eta_{i}\right]$, se $x \in X$ satisfaz $d\left(x, z_{\eta}^{*}\right)<\delta$, então ou $T_{\eta}(t) x \rightarrow z_{\eta}^{*}$ quando $t \rightarrow \infty$, ou $d\left(T_{\eta}(t) x, \mathcal{M}_{(i-1), \eta}\right) \rightarrow 0$ quando $t \rightarrow \infty$.

Demonstração: Para o caso em que $z_{0}^{*} \in \mathcal{N}_{1,0}$, suponhamos que a conclusão não se verifique, donde segue-se a existência de sequências $\eta_{k} \rightarrow 0^{+}$em $(0,1],\left(x_{k}\right)_{k \in \mathbb{N}}$ em $X$ e $\left(z_{k}^{*}\right)_{k \in \mathbb{N}}$ com $z_{k}^{*} \in \mathcal{E}_{\eta_{k}}$ e $d\left(x_{k}, z_{k}^{*}\right)<\frac{1}{k}$ tais que $T_{\eta_{k}}(t) x_{k}$ não converge a $z_{k}^{*}$ quando $t \rightarrow \infty$, para todo natural $k$.

Fixemos $\delta_{0}>0$ tal que $\mathcal{O}_{\delta_{0}}\left(z_{i, \eta}^{*}\right) \cap \mathcal{O}_{\delta_{0}}\left(z_{j, \eta}^{*}\right)=\varnothing$ para $i \neq j$ e $\eta$ suficientemente pequeno. Então, como cada $\left\{T_{\eta}(t): t \geq 0\right\}$ é de tipo gradiente, temos $d\left(T_{\eta_{k}}(t) x_{k}, \mathcal{E}_{\eta_{k}} \backslash\left\{z_{k}^{*}\right\}\right) \underset{t \rightarrow \infty}{\rightarrow} 0$ e podemos, portanto, para $k$ suficientemente grande, 
encontrar $\tau_{k}>0$ de modo que

$$
\begin{gathered}
d\left(T_{\eta_{k}}(t) x_{k}, z_{k}^{*}\right)<\delta_{0} \text { para } t \in\left[0, \tau_{k}\right) \text { e } \\
d\left(T_{\eta_{k}}\left(\tau_{k}\right) x_{k}, z_{k}^{*}\right)=\delta_{0} .
\end{gathered}
$$

Da convergência $T_{\eta} \underset{\eta \rightarrow 0^{+}}{\rightarrow} T_{0}$ uniforme sobre compactos de $[0, \infty) \times X$ e de (6.2.4) é fácil ver que $\tau_{k} \underset{k \rightarrow \infty}{\rightarrow} \infty$. Consideremos então, para cada $k$, a aplicação $\xi_{k}:\left[-\tau_{k}, \infty\right) \rightarrow$ $X$ dada por $\xi_{k}(t):=T_{\eta_{k}}\left(t+\tau_{k}\right) x_{k} t \in\left[-\tau_{k}, \infty\right)$. Usando argumentos já usuais e (6.2.3) concluímos a existência de uma solução global limitada $\xi_{0}: \mathbb{R} \rightarrow X$, para $\left\{T_{0}(t): t \geq 0\right\}$, tal que $\lim _{k \rightarrow \infty} \xi_{k}(t)=\xi_{0}(t)$ para todo $t \in \mathbb{R}$ e $\lim _{t \rightarrow-\infty} \xi_{0}(t)=z_{0}^{*}$. Mas, estando $z_{0}^{*}$ em $\mathcal{N}_{1,0}$ com $\left\{T_{0}(t): t \geq 0\right\}$ de tipo gradiente, $\xi_{0}$ deve ser constante com $\xi_{0}(t)=z_{0}^{*}$ para todo $t$ real, contradizendo o fato de que $d\left(\xi_{0}(0), z_{0}^{*}\right)=\delta_{0}$, que resulta de (6.2.4) após fazer $k \rightarrow \infty$, e assim nossa afirmação está correta.

Para a demonstração dos demais casos procede-se analogamente ao que fizemos na prova do Lema 6.1.4.

Com a ajuda deste último lema podemos provar o resultado sobre perturbação de níveis.

Teorema 6.2.2. Além das hipóteses do lema anterior, sejam $\mathcal{N}_{1, \eta}, \cdots, \mathcal{N}_{n(\eta), \eta}$ para $\eta \in(0,1]$ os níveis de energia do semigrupo de tipo gradiente $\left\{T_{\eta}(t): t \geq 0\right\}$ e suponhamos que, sempre que $\left(\eta_{k}\right)_{k \in \mathbb{N}}$ é uma sequência em $[0,1]$ com $\eta_{k} \underset{k \rightarrow \infty}{\rightarrow} 0^{+} e$ $\left(z_{\eta_{k}}^{*}\right)_{k \in \mathbb{N}}$ são tais que, para algum $i \in \bigcap_{\eta \in[0,1]}\{1,2, \cdots, n(\eta)\}, z_{\eta_{k}}^{*} \in \mathcal{N}_{i, \eta_{k}}$ para todo natural $k$ e $z_{\eta_{k}}^{*} \underset{k \rightarrow \infty}{\rightarrow} z_{0}^{*}$ então $z_{0}^{*} \in \mathcal{N}_{i, 0}$.

Nestas condições, se n é o número máximo de níveis de energia para $\left\{T_{0}(t): t \geq 0\right\}$, sendo estes $\mathcal{N}_{1,0}, \cdots, \mathcal{N}_{n, 0}$, então existe $\eta^{*} \in(0,1]$ tal que para todo $\eta \in\left(0, \eta^{*}\right]$ o semigrupo $\left\{T_{\eta}(t): t \geq 0\right\}$ possui exatamente $n$ níveis de energia, $\mathcal{N}_{1, \eta}, \cdots, \mathcal{N}_{n, \eta}$ (ou seja, $n(\eta)=n$ para todo $\left.\eta \in\left[0, \eta^{*}\right]\right)$, os quais se comportam continuamente quando $\eta \rightarrow 0^{+}$, isto é, para todo $i=1,2, \cdots, n$ tem-se

$$
d_{H}\left(\mathcal{N}_{i, \eta}, \mathcal{N}_{i, 0}\right) \underset{\eta \rightarrow 0^{+}}{\rightarrow} 0
$$

Demonstração: Com efeito, digamos que os níveis de energia do semigrupo limite estejam dados por $\mathcal{N}_{i, 0}=\left\{z_{l_{1}, 0}^{(i) *}, \cdots, z_{l_{k(i)}, 0}^{(i) *}\right\}$ para $i=1,2, \cdots, n$. Definindo para cada $\eta \in(0,1]$ e cada $i=1,2, \cdots, n$ o conjunto $\mathcal{H}_{i, \eta}:=\left\{z_{l_{1}, \eta}^{(i) *}, \cdots, z_{l_{k(i)}, \eta}^{(i) *}\right\}$, diretamente das hipóteses do Teorema 3.2.4, tem-se para cada $i=1,2, \cdots, n, d_{H}\left(\mathcal{H}_{i, \eta}, \mathcal{N}_{i, 0}\right) \underset{\eta \rightarrow 0^{+}}{\rightarrow} 0$. É claro que os conjuntos $\mathcal{H}_{i, \eta}$ 's são os candidatos naturais a níveis de energia dos 
semigrupos perturbados $T_{\eta}(\cdot)$, portanto, provemos que, efetivamente, $\mathcal{H}_{i, \eta}=\mathcal{N}_{i, \eta}$, para $i=1,2, \cdots, n$ e $\eta$ suficientemente pequeno.

De fato, por um lado, para $i=1$ consideremos a família $\left(z_{l_{1}, \eta}^{(1) *}\right)_{\eta \in(0,1]}$ formada pelos primeiros elementos dos conjuntos $\mathcal{H}_{1, \eta}$. Afirmamos que existe $\theta_{1} \in(0,1]$ tal que $z_{l_{1}, \eta}^{(1) *} \in \mathcal{N}_{1, \eta}$ para todo $\eta \in\left(0, \theta_{1}\right]$. Caso contrário, podemos encontrar uma sequência $\eta_{k} \rightarrow 0^{+}$e soluções globais não constantes $\xi_{k}: \mathbb{R} \rightarrow X$ para $\left\{T_{\eta_{k}}(t): t \geq 0\right\}$ tais que $\lim _{t \rightarrow-\infty} \xi_{k}(t)=z_{l_{1}, \eta_{k}}^{(1) *}$ e $\lim _{t \rightarrow \infty} \xi_{k}(t)=w_{k}^{*}$, para certos equilíbrios $w_{k}^{*} \in \mathcal{E}_{\eta_{k}}$ com $w_{k}^{*} \neq z_{l_{1}, \eta_{k}}^{(1) *}$ para todo $k$.

Escolhamos agora, para cada natural $k$, um número real $\tau_{k}<0$ de modo que $d\left(\xi_{k}(t), z_{l_{1}, \eta_{k}}^{(1) *}\right)<\delta_{0}$ para todo $t<\tau_{k} \mathrm{e}$

$$
d\left(\xi_{k}\left(\tau_{k}\right), z_{l_{1}, \eta_{k}}^{(1) *}\right)=\delta_{0}
$$

onde $\delta_{0}>0$ é tal que $\mathcal{O}_{\delta_{0}}\left(z_{i, \eta}^{*}\right) \cap \mathcal{O}_{\delta_{0}}\left(z_{j, \eta}^{*}\right)=\varnothing$ para $i \neq j$ e $\eta$ suficientemente pequeno.

Definindo para cada $k, \zeta_{k}: \mathbb{R} \rightarrow X$ por $\zeta_{k}(t):=\xi_{k}\left(t+\tau_{k}\right) t \in \mathbb{R}$, por argumentos usuais, obtém-se $\zeta_{0}: \mathbb{R} \rightarrow X$ solução global de $\left\{T_{0}(t): t \geq 0\right\}$ satisfazendo $\lim _{t \rightarrow-\infty} \zeta_{0}(t)=$ $z_{l_{1}, 0}^{(1) *} \in \mathcal{N}_{1,0}$. Da definição de $\mathcal{N}_{1,0}$ segue-se que $\zeta_{0}$ deve ser solução constante, donde $\zeta_{0}(t)=z_{l_{1}, 0}^{(1) *}$ para todo $t \in \mathbb{R}$. Mas isto contradiz $d\left(\zeta_{0}(0), z_{l_{1}, 0}^{(1) *}\right)=\delta_{0}$, que resulta de (6.2.5) após fazer $k \rightarrow \infty$. Repetindo este raciocínio para $j=2, \cdots, k(1)$ obteremos $\eta_{1} \in(0,1]$ tal que $z_{l_{j}, \eta}^{(1) *} \in \mathcal{N}_{1, \eta}$ para $j=1, \cdots, k(1)$ e $\eta \in\left(0, \eta_{1}\right]$, isto é, $\mathcal{H}_{1, \eta} \subset \mathcal{N}_{1, \eta}$ para todo $\eta \in\left(0, \eta_{1}\right]$.

Por outro lado, existe $\eta_{1}^{\prime} \in\left(0, \eta_{1}\right]$ tal que $\mathcal{N}_{1, \eta} \subset \mathcal{H}_{1, \eta}$ sempre que $\eta \in\left(0, \eta_{1}^{\prime}\right]$. Porque, caso contrário, existiriam uma sequência $\eta_{k} \rightarrow 0^{+}$e, para cada natural $k$, um ponto de equilíbrio $z_{\eta_{k}}^{*} \in \mathcal{N}_{1, \eta_{k}} \backslash \mathcal{H}_{1, \eta_{k}}$ tal que a sequência $\left(z_{\eta_{k}}^{*}\right)_{k \in \mathbb{N}}$ convergiria para $z_{0}^{*}$ quando $k \rightarrow \infty$. Entretanto, das hipóteses, deveriamos ter $z_{0}^{*} \in \mathcal{N}_{1,0}$, o que contraria $z_{\eta_{k}}^{*} \notin \mathcal{H}_{1, \eta_{k}}$ qualquer que seja $k$. Unindo este último fato ao anteriormente demonstrado, conclui-se que $\mathcal{H}_{1, \eta}=\mathcal{N}_{1, \eta}$ para $\eta \in\left(0, \eta_{1}^{\prime}\right]$.

Agora, mostremos que existe $\eta_{2} \in\left(0, \eta_{1}^{\prime}\right]$ tal que $\mathcal{H}_{2, \eta} \subset \mathcal{M}_{2, \eta}$ sempre que $\eta \in\left(0, \eta_{2}\right]$. Antes de fazer a prova, notemos que se esta afirmação é correta, pelo resultado já provado acima para o nível um, teremos, na verdade, que $\mathcal{H}_{2, \eta} \subset \mathcal{M}_{2, \eta} \backslash \mathcal{N}_{1, \eta}=\mathcal{N}_{2, \eta}$, uma vez que $\mathcal{H}_{2, \eta}$ é disjunto de $\mathcal{H}_{1, \eta}=\mathcal{N}_{1, \eta}$ para todo $\eta \in\left(0, \eta_{2}\right]$.

Para provar a existência de um tal $\eta_{2}$, tomemos a família $\left(z_{l_{1}, \eta}^{(2) *}\right)_{\eta \in\left(0, \eta_{1}\right]}$. Afirmamos que existe $\theta_{2} \in\left(0, \eta_{1}\right]$ tal que $z_{l_{1}, \eta}^{(2) *} \in \mathcal{M}_{2, \eta}$ para todo $\eta \in\left(0, \theta_{2}\right]$. De outro modo, (recordando o caso anterior, temos $z_{l_{1}, \eta}^{(2) *} \notin \mathcal{N}_{1, \eta}=\mathcal{H}_{1, \eta}$ para todo $\eta$ suficientemente pequeno) obtém-se uma sequência $\eta_{k} \rightarrow 0^{+}$e correspondentes soluções globais não constantes $\xi_{k}: \mathbb{R} \rightarrow X$ para $\left\{T_{\eta_{k}}(t): t \geq 0\right\}$ tais que $\lim _{t \rightarrow-\infty} \xi_{k}(t)=z_{l_{1}, \eta_{k}}^{(2) *}$ e $\lim _{t \rightarrow \infty} \xi_{k}(t)=w_{k}^{*}$, com $w_{k}^{*} \notin \mathcal{M}_{1, \eta_{k}}=\mathcal{N}_{1, \eta_{k}}$ e, além disso, $w_{k}^{*} \neq z_{l_{1}, \eta_{k}}^{(2) *}$ para todo $k$. Como na etapa anterior e 
com o mesmo $\delta_{0}$ lá considerado, sejam, para cada $k, \tau_{k}<0$ tal que $d\left(\xi_{k}(t), z_{l_{1}, \eta_{k}}^{(2) *}\right)<\delta_{0}$ para todo $t<\tau_{k}$ e $d\left(\xi_{k}\left(\tau_{k}\right), z_{l_{1}, \eta_{k}}^{(2) *}\right)=\delta_{0}$.

Sejam, de acordo com o Lema $6.2 .1, \delta>0$ e $\bar{\eta}_{1} \in\left(0, \eta_{1}^{\prime}\right]$ tais que a estabilidade assintótica dos elementos de $\mathcal{N}_{1, \eta}$ se verifica em $\mathcal{O}_{\delta}\left(\mathcal{N}_{1, \eta}\right)$ sempre que $\eta \in\left[0, \bar{\eta}_{1}\right]$. Então, é claro que

$$
d\left(\xi_{k}(t), \mathcal{N}_{1, \eta_{k}}\right) \geq \delta \text {, para todo } t \in \mathbb{R} \text { e todo } k \in \mathbb{N} \text {. }
$$

Definindo as soluções $\zeta_{k}: \mathbb{R} \rightarrow X$ por $\zeta_{k}(t):=\xi_{k}\left(t+\tau_{k}\right) t \in \mathbb{R}$, novamente obtemos uma solução global $\zeta_{0}: \mathbb{R} \rightarrow X$ de $\left\{T_{0}(t): t \geq 0\right\}$ tal que $\lim _{k \rightarrow \infty} \zeta_{k}(t)=\zeta_{0}(t)$ para todo $t \in \mathbb{R}$ com $\lim _{t \rightarrow-\infty} \zeta_{0}(t)=z_{l_{1}, 0}^{(2) *} \in \mathcal{N}_{2,0}$ e, consequentemente, $\lim _{t \rightarrow \infty} \zeta_{0}(t)=w_{0}^{*}$ para algum $w_{0}^{*} \in \mathcal{N}_{1,0}$. Fixemos $\tau>0$ de maneira que $d\left(\zeta_{0}(\tau), w_{0}^{*}\right)<\frac{\delta}{2}$, daí segue a existência de $k_{0} \in \mathbb{N}$ tal que $d\left(\xi_{k}\left(\tau+\tau_{k}\right), \mathcal{N}_{1, \eta_{k}}\right)<\delta$ para todo $k \geq k_{0}$, o que naturalmente contradiz (6.2.6) acima e a afirmação segue.

O argumento anterior pode ser repetido para todo $j=2, \cdots, k(2)$ e, após ser efetuado, obteremos $\eta_{2} \in(0,1]$ tal que $\mathcal{H}_{2, \eta} \subset \mathcal{M}_{2, \eta}$ sempre que $\eta \in\left(0, \eta_{2}\right]$, como queríamos.

Analogamente ao que fizemos no caso do nível um, conseguiremos $\eta_{2}^{\prime} \in\left(0, \eta_{2}\right]$ tal que $\mathcal{N}_{2, \eta} \subset \mathcal{H}_{2, \eta}$ sempre que $\eta \in\left(0, \eta_{2}^{\prime}\right]$, donde conclui-se que $\mathcal{H}_{2, \eta}=\mathcal{N}_{2, \eta}$ para todos os $\eta \in\left(0, \eta_{2}^{\prime}\right]$.

Finalmente, repetindo os argumentos realizados anteriormente para os índices restantes $i=3, \cdots, n$ e observando que $\mathcal{E}_{\eta}=\mathcal{H}_{1, \eta} \cup \cdots \cup \mathcal{H}_{n, \eta}$ para cada $\eta$, nosso resultado fica demonstrado.

O seguinte resultado nos mostra uma condição bastante razoável para se verificar a validez de uma das hipóteses exigida no teorema anterior (a saber, a que é adicional às hipóteses do Teorema 3.2.4). Ele afirma que, quando as conexões entre quaisquer dois pontos de equilíbrio de um semigrupo de tipo gradiente são mantidas por perturbação, então a condição (adicional) se verifica e, consequentemente, a conclusão do teorema anterior também.

Mais precisamente, consideremos $\left\{T_{\eta}(t): t \geq 0\right\}_{\eta \in[0,1]}$ uma família de semigrupos de tipo gradiente tais que, para cada $\eta \in[0,1], T_{\eta}(\cdot)$ possui atrator global $\mathcal{A}_{\eta}$ e conjunto de equilíbrios $\mathcal{E}_{\eta}:=\left\{z_{1, \eta}^{*}, \cdots, z_{p, \eta}^{*}\right\}$. Nestas condições suponhamos que:

(H) Para todo par $z^{*}$ e $w^{*}$ de pontos de $\mathcal{E}_{0} \operatorname{com} z^{*} \succ w^{*}$, se $z_{\eta}^{*}$ e $w_{\eta}^{*}$ estão em $\mathcal{E}_{\eta}$ e são tais que $z_{\eta}^{*} \underset{\eta \rightarrow 0^{+}}{\rightarrow} z^{*}$ e $w_{\eta}^{*} \underset{\eta \rightarrow 0+}{\rightarrow} w^{*}$, então $z_{\eta}^{*} \succ w_{\eta}^{*}$ para todo $\eta$ suficientemente pequeno.

Temos o seguinte critério. 
Teorema 6.2.3. Suponhamos, adicionalmente às hipóteses do Teorema 3.2.4, que a condição $(H)$ acima se verifique para uma família de semigrupos $\left\{T_{\eta}(t): t \geq 0\right\}_{\eta \in[0,1]}$.

Mantendo as mesmas notações do Teorema 6.2.2, se $\left(\eta_{k}\right)_{k \in \mathbb{N}}$ é uma sequência em $[0,1] \operatorname{com} \eta_{k} \underset{k \rightarrow \infty}{\rightarrow} 0^{+}$e para algum $i \in \bigcap_{\eta \in[0,1]}\{1,2, \cdots, n(\eta)\}\left(z_{\eta_{k}}^{*}\right)_{k \in \mathbb{N}}$ é uma sequência com $z_{\eta_{k}}^{*} \in \mathcal{N}_{i, \eta_{k}}$ para todo $k$ e $z_{\eta_{k}}^{*} \underset{k \rightarrow \infty}{\rightarrow} z^{*}$ para algum $z^{*} \in \mathcal{E}_{0}$, então $z^{*} \in \mathcal{N}_{i, 0}$.

Demonstração: Com efeito, se fosse $i=1$ e $z^{*}$ não pertencesse a $\mathcal{N}_{1,0}$, existiria $w^{*} \in \mathcal{E}_{0} \operatorname{com} w^{*} \neq z^{*}$ tal que $z^{*} \succ w^{*}$. Seja então, $\left(w_{\eta_{k}}^{*}\right)_{k \in \mathbb{N}}$ a única sequência com $w_{\eta_{k}}^{*} \in \mathcal{E}_{\eta_{k}}$, para todo $k$, tal que $w_{\eta_{k}}^{*} \underset{k \rightarrow \infty}{\rightarrow} w^{*}$. Pela hipótese $(H)$ temos $z_{\eta_{k}}^{*} \succ$ $w_{\eta_{k}}^{*}$ para todo $k$ suficientemente grande, mas, como $z_{\eta_{k}}^{*} \in \mathcal{N}_{1, \eta_{k}}$ para cada natural $k$, obrigatoriamente $z_{\eta_{k}}^{*}=w_{\eta_{k}}^{*}$, donde se infere que $z^{*}=w^{*}$, o que é obviamente uma contradição. Daí temos que o teorema é válido para $i=1$, e se unimos este fato ao que fizemos na prova da primeira parte do Teorema 6.2.2, obteremos a existência de $\eta_{1} \in(0,1]$ tal que os conjuntos $\mathcal{H}_{1, \eta}$, como definidos em tal teorema, coincidem com os conjuntos $\mathcal{N}_{1, \eta}$ para $\eta \in\left(0, \eta_{1}\right]$.

Agora, se tivermos $i=2$ mas com $z^{*}$ não pertencente a $\mathcal{N}_{2,0}=\mathcal{M}_{2,0} \backslash \mathcal{N}_{1,0}$ teremos, em primeiro lugar, que tampouco $z^{*}$ pode estar em $\mathcal{N}_{1,0}$, porque se $z^{*} \in \mathcal{N}_{1,0}$, como vimos acima $\mathcal{N}_{1, \eta}=\mathcal{H}_{1, \eta}$ e por isto $d_{H}\left(\mathcal{N}_{1, \eta}, \mathcal{N}_{1,0}\right) \underset{\eta \rightarrow 0^{+}}{\rightarrow} 0$, o que obriga $z_{\eta_{k}}^{*}$ a estar em $\mathcal{N}_{1, \eta_{k}}$ para todo $k$ suficientemente grande, contradizendo $z_{\eta_{k}}^{*} \in \mathcal{N}_{2, \eta_{k}}$ para todo $k$.

Nestas condições, $z^{*} \in \mathcal{N}_{3,0} \cup \mathcal{N}_{4,0} \cup \cdots \cup \mathcal{N}_{n, 0}$ e portanto podemos encontrar $w^{*} \in \mathcal{E}_{0}$ com $z^{*} \succ w^{*}$ tal que $w^{*}$ não está em $\mathcal{N}_{1,0}$. Seja $\left(w_{\eta_{k}}^{*}\right)_{k \in \mathbb{N}}$ a única sequência com $w_{\eta_{k}}^{*} \in \mathcal{E}_{\eta_{k}}$, para todo $k$, tal que $w_{\eta_{k}}^{*} \underset{k \rightarrow \infty}{\rightarrow} w^{*}$. Pela hipótese $(H)$ devemos ter $z_{\eta_{k}}^{*} \succ$ $w_{\eta_{k}}^{*}$ para todo $k$ suficientemente grande, mas como $z_{\eta_{k}}^{*} \in \mathcal{N}_{2, \eta_{k}}$ para cada natural $k$, necessariamente $w_{\eta_{k}}^{*} \in \mathcal{N}_{1, \eta_{k}}$ para cada $k$, mas da parte já provada acima reultaria que $w^{*}$ deveria estar em $\mathcal{N}_{1,0}$, contradizendo sua escolha e provando o caso $i=2$. Agora, unindo este resultado ao que fizemos na prova da segunda parte do Teorema 6.2.2, obteremos a existência de $\eta_{2} \in\left(0, \eta_{1}\right]$ tal que os conjuntos $\mathcal{H}_{2, \eta}$, como definidos no teorema anterior, coincidem com os conjuntos $\mathcal{N}_{2, \eta}$ para $\eta \in\left(0, \eta_{1}\right]$, donde vem, em particular, que $d_{H}\left(\mathcal{N}_{2, \eta}, \mathcal{N}_{2,0}\right) \underset{\eta \rightarrow 0^{+}}{\rightarrow} 0$.

Consideremos agora o caso $i=3$ e, novamente por contradição, suponhamos que $z^{*}$ não está em $\mathcal{N}_{3,0}$. Por motivo análogo ao considerado no caso anterior, tem-se, além disso, que $z^{*}$ não está em $\mathcal{N}_{1,0} \cup \mathcal{N}_{2,0}=\mathcal{M}_{2,0}$, pois como $d_{H}\left(\mathcal{N}_{i, \eta}, \mathcal{N}_{i, 0}\right) \underset{\eta \rightarrow 0^{+}}{\rightarrow} 0$ para $i=1$ e 2, se $z^{*} \in \mathcal{N}_{1,0} \cup \mathcal{N}_{2,0}$ teriamos que $z_{\eta_{k}}^{*} \in \mathcal{N}_{1, \eta_{k}} \cup \mathcal{N}_{2, \eta_{k}}$ para todo $k$ suficientemente grande, o que contradiz $z_{\eta_{k}}^{*} \in \mathcal{N}_{3, \eta_{k}}$ para todo $k$.

Assim, $z^{*} \in \mathcal{N}_{4,0} \cup \cdots \cup \mathcal{N}_{n, 0}$ e portanto pode-se encontrar $w^{*} \in \mathcal{E}_{0}$ com $z^{*} \succ w^{*}$ tal que $w^{*}$ não está em $\mathcal{M}_{2,0}$. Analogamente aos casos anteriores, seja $\left(w_{\eta_{k}}^{*}\right)_{k \in \mathbb{N}}$ a sequência com $w_{\eta_{k}}^{*} \in \mathcal{E}_{\eta_{k}}$, para todo $k$, tal que $w_{\eta_{k}}^{*} \underset{k \rightarrow \infty}{\rightarrow} w^{*}$. Segundo a hipótese $(H)$ 
temos $z_{\eta_{k}}^{*} \succ w_{\eta_{k}}^{*}$ para todo $k$ suficientemente grande, mas sendo $z_{\eta_{k}}^{*} \in \mathcal{N}_{3, \eta_{k}}$ para cada natural $k$, necessariamente $w_{\eta_{k}}^{*} \in \mathcal{M}_{2, \eta_{k}}$ para cada $k$, mas das partes já demonstradas anteriormente teríamos que $w^{*}$ deveria estar em $\mathcal{N}_{1,0} \cup \mathcal{N}_{2,0}=\mathcal{M}_{2,0}$, contradizendo a maneira com a qual foi escolhido e demonstrando o caso $i=3$.

O procedimento para se fazer os demais casos está claro do que acabamos de fazer e o mesmo deve terminar após um número finito de etapas, porque o conjunto $\bigcap_{\eta \in[0,1]}\{1,2, \cdots, n(\eta)\}$ é finito, completando a prova. 



\section{Capítulo}

7

\section{Decomposição de Morse não autônoma}

Neste capítulo apresentamos, de forma introdutória, alguns dos principais conceitos relacionados à noção de processo de evolução não autônomo e estabelecemos suas propriedades mais elementares, com o objetivo de estender a maioria dos resultados prévios, também para o caso não autônomo, principalmente as noções de decomposição de Morse e função de Lyapunov.

Pretendemos aqui, discutir algumas das principais diferenças que existem entre as duas naturezas de sistemas dinâmicos, enfatizando que no caso não autônomo é necessário, muitas vezes, impor certas restrições sobre o comportamento do sistema a fim de que se possa estabelecer, de forma consistente, as propriedades mais naturais.

Vimos nos capítulos anteriores que, partindo da decomposição de Morse, adequadamente definida, para um semigrupo de tipo gradiente, foi possível construir uma função de Lyapunov para ele e então demonstrar que os mesmos são semigrupos gradientes. Contudo, para o caso não autônomo não existe, até o momento, um conceito semelhante de "função de Lyapunov", o que existem são os processos de evolução de tipo gradiente (veja a Definição 7.2.4 abaixo) e, como veremos a seguir, podemos repetir, fazendo as devidas adaptações, muitos dos argumentos que fizemos para o caso autônomo e, desta forma, obter uma definição apropriada de função de Lyapunov para processos de evolução e, além disso, fornecer condições para que os processos de tipo gradiente possuam uma tal função. Entretanto, como mencionamos acima, para a construção que faremos a seguir será necessário impor algumas restrições sobre a dinâmica de certos 
objetos associados ao processo. Não obstante, na Seção 7.5, apresentamos uma classe de exemplos onde se encontram satisfeitas as restrições exigidas na teoria abstrata, completando assim a discusão.

Muitos dos resultados que desenvolvemos neste capítulo convertem seus análogos dos capítulos anteriores em casos particulares mas, acreditamos que, tratando os dois casos separadamente, a exposição se torna mais simples, além de refletir o fato de que o desenvolvimento da teoria não autônoma é, em muitos aspectos, inspirado na teoria autônoma. Além disso, a teoria autônoma é mais intuitiva e a não autônoma requer uma abordágem mais técnica, o que no caso autônomo não faz-se necessário.

Para levar a cabo nossa tarefa, faz-se necessário a introdução de mais algumas definições e notações.

\subsection{Processos de evolução}

Neste capítulo trabalharemos com sistemas dinâmicos segundo a definição abaixo.

Definição 7.1.1. Seja $X$ um espaço métrico. Um processo de evolução em $X$, ou simplesmente um processo, é uma família a dois parâmetros $\{T(t, s): t \geq s \in \mathbb{R}\}$ de aplicações contínuas de $X$ em si mesmo, que as vezes denota-se simplesmente por $T(\cdot, \cdot)$, satisfazendo as seguintes três propriedades:

(i) $T(t, t)=I$, qualquer que seja o real $t$, sendo $I: X \rightarrow X$ a identidade de $X$.

(ii) $T(t, \tau) T(\tau, s)=T(t, s)$, sempre que $t \geq \tau \geq s$ são reais dados.

(iii) Pondo $\mathcal{P}:=\left\{(t, s) \in \mathbb{R}^{2}: t \geq s\right\}$, a aplicação $\mathcal{P} \times X \ni((t, s), x) \mapsto T(t, s) x \in$ $X$ é contínua, onde o conjunto $\mathcal{P} \times X$ está dotado da topologia produto.

Processos de evolução são também chamados de sistemas dinâmicos não autônomos e aqui, usaremos indistintamente as duas nomenclaturas. Também, as notações $\{T(t, s): t \geq s\}$ e $T(\cdot, \cdot)$ serão, por simplicidade, adotadas para indicar o processo de evolução $\{T(t, s): t \geq s \in \mathbb{R}\}$.

Observemos que, se $\{S(t): t \geq 0\}$ é um semigrupo em $X$, a ele está associado o processo de evolução $\{T(t, s): t \geq s\}$ dado por

$$
T(t, s):=S(t-s) \text { para } t \geq s .
$$

É fácil ver que a família $T(\cdot, \cdot)$, definida acima, satisfaz as condições $(i),($ ii $)$ e $($ iii $)$ da Definição 7.1.1. Reciprocamente, se $\{T(t, s): t \geq s\}$ é um processo de evolução tal que $T(t, s)=T(t-s, 0)$ sempre que $t \geq s$, resulta imediatamente que a família $\{S(t): t \geq 0\}$, definida por

$$
S(t):=T(t, 0), \text { para } t \geq 0,
$$


é um semigrupo em $X$.

Em ambos os casos acima, diz-se que $\{T(t, s): t \geq s\}$ é um processo de evolução autônomo.

Segue-se daí que, o fato crucial que distingue as duas naturezas de sistemas dinâmicos é a dependência explícita do tempo inicial na evolução dos estados. Em outras palavras, no caso autônomo a evolução de um estado $x \in X$ no intervalo de tempo $[s, t]$ depende somente do tempo decorrido $t-s$, enquanto que este não é o caso, em geral, para sistemas não autônomos.

Uma aplicação $\xi: \mathbb{R} \rightarrow X$ chama-se uma solução global para o processo $T(\cdot, \cdot)$ quando

$$
T(t, s) \xi(s)=\xi(t) \text { toda vez que } t \geq s
$$

ou seja, quando o processo $T(\cdot, \cdot)$ é o responsável por fazer evoluir os valores que $\xi$ assume.

Como antes, se $\xi: \mathbb{R} \rightarrow X$ é uma solução global de $T(\cdot, \cdot)$ então $\xi$ é contínua. De fato, fixados $t_{0} \in \mathbb{R}$ e $\delta>0$ se $t$ é um real qualquer em $\left(t_{0}-\delta, t_{0}+\delta\right)$, da definição de solução, podemos escrever

$$
d\left(\xi(t), \xi\left(t_{0}\right)\right)=d\left(T(t, s) \xi(s), T\left(t_{0}, s\right) \xi(s)\right)
$$

onde $s$ é escolhido menor do que $t_{0}-\delta$, donde a continuidade de $\xi$ segue da propriedade (iii) acima.

Pretendemos, a seguir, definir um conceito de "atrator" para processos de evolução que seja, em algum sentido, uma extensão do conceito de atrator global previamente estudado para os semigrupos nos capítulos anteriores. Para o caso não autônomo, a noção de atração que adotamos aqui é a em sentido pullback, sem mencionar nenhuma outra (para outras noções de atração veja [6]).

Comecemos definindo o que se entende por família invariante.

Definição 7.1.2. Sejam $\{T(t, s): t \geq s\}$ um processo de evolução e $A:=\{A(t): t \in$ $\mathbb{R}\}$ uma família de subconjuntos de $X$.

Diz-se que A é uma família invariante (pelo processo $T(\cdot, \cdot)$ ) quando para todos os reais $t \geq s$ tem-se

$$
T(t, s) A(s)=A(t)
$$

Quando $A=\{A(t): t \in \mathbb{R}\}$ é uma família invariante, o conjunto

$$
\Gamma:=\bigcup_{t \in \mathbb{R}} A(t),
$$

chama-se o traço de A. 
Aqui, como é evidente, podemos observar que existe uma diferença entre a noção de invariância para os casos autônomo e não autônomo. No primeiro caso, tinhamos um conjunto $A$ fixado, enquanto que no segundo, temos uma família a um parâmetro de conjuntos, e assim podemos verificar que a segunda é mais fraca do que a primeira no sentido de que, se $A$ é um conjunto invariante por um semigrupo $T(\cdot)$, tem-se que, definindo para cada real $t, A(t):=A$, a família constante $\{A(t): t \in \mathbb{R}\}$ é invariante pelo processo $\{T(t-s): t \geq s\}$ segundo a Definição 7.1.2, mas, não é verdade em geral, que se uma família $\left\{A^{\prime}(t): t \in \mathbb{R}\right\}$ é invariante por $\{T(t-s): t \geq s\}$, no sentido da Definição 7.1.2, então $A^{\prime}(t)=A^{\prime}$ para todo real $t$ e um certo subconjunto fixo $A^{\prime}$. Com efeito, tomando $\xi: \mathbb{R} \rightarrow X$ uma solução global não constante de $\{T(t-s)$ : $t \geq s\}$ (supondo, evidentemente, que uma tal solução existe), resulta que a família de conjuntos unitários $\{\{\xi(t)\}: t \in \mathbb{R}\}$ é invariante segundo a Definição 7.1.2, mas não é uma famíla constante.

Observemos que é possível provar, para famílias invariantes, um resultado análogo à Proposição 1.1.2, mais precisamente tem-se a

Proposição 7.1.3. Sejam $\{T(t, s): t \geq s\}$ um processo de evolução e $A:=\{A(t): t \in$ $\mathbb{R}\}$ uma família de subconjuntos de $X$. Então, A é invariante se, e somente se, para cada real s e cada ponto $x \in A(s)$ existe uma solução global $\xi: \mathbb{R} \rightarrow X$ de $T(\cdot, \cdot)$ com $\xi(s)=x$ e $\xi(t) \in A(t)$ para todo real $t$.

Demonstração: Semelhante à prova da Proposição 1.1.2.

Agora definimos a noção de atração em sentido pullback como segue.

Definição 7.1.4. Dado $t \in \mathbb{R}$, diz-se que um subconjunto $A(t)$ de $X$ atrai um subconjunto $B$ de $X$ em sentido pullback e no instante $t \in \mathbb{R}$ quando

$$
\lim _{s \rightarrow-\infty} \operatorname{dist}(T(t, s) B, A(t))=0
$$

ou, dito de outra forma, quando para todo $\varepsilon>0$, existe um real $s_{0}=s_{0}(B, \varepsilon) \leq t$ tal que

$$
T(t, s) B \subset \mathcal{O}_{\varepsilon}(A(t)) \text { sempre que } s \leq s_{0} .
$$

Finalmente, introduzimos o conceito de atrator pullback.

Definição 7.1.5. Seja $\{T(t, s): t \geq s\}$ um processo de evolução em um espaço métrico $X$. Uma familia $\mathcal{A}:=\{\mathcal{A}(t): t \in \mathbb{R}\}$ de subconjuntos de $X$ chama-se um atrator pullback para $T(\cdot, \cdot)$ quando cumpre as seguintes quatro condições:

(i) Cada $\mathcal{A}(t)$ é um subconjunto compacto de $X$. 
(ii) A família $\mathcal{A}$ é invariante pelo processo $T(\cdot, \cdot)$.

(iii) Para cada número real t o conjunto $\mathcal{A}(t)$ atrai cada um dos subconjuntos limitados de $X$ em sentido pullback e no instante $t$.

(iv) Se $\mathcal{B}:=\{\mathcal{B}(t): t \in \mathbb{R}\}$ é uma familia de subconjuntos fechados de $X$ tal que, para cada número real $t, \mathcal{B}(t)$ atrai cada um dos limitados de $X$ em sentido pullback e no instante $t$, então $\mathcal{A}(t) \subset \mathcal{B}(t)$ para todo real $t$.

Façamos agora alguns comentários acerca das definições anteriores.

Em primeiro lugar, a diferença evidente entre a noção de atrator pullback e a de atrator autônomo é o fato de que, aqui, o atrator não é, necessariamente, composto por um único conjunto fixo e a idéia que está por trás da atração pullback é que, para cada instante fixado $t$ tem-se o controle das evoluções dos estados até o tempo final $t$ sempre que se toma instantes iniciais $s$ (infinitamente) grandes negativos, enquanto que o atrator autônomo fornece o controle das evoluções para tempos finais (infinitamente) grandes positivos.

Observemos, além disso, que no caso pullback foi necessário impor a condição de minimalidade $(i v)$ porque, assim, pode-se garantir a unicidade do atrator pullback. Com efeito, se $\{\mathcal{A}(t): t \in \mathbb{R}\}$ e $\left\{\mathcal{A}^{\prime}(t): t \in \mathbb{R}\right\}$ fossem dois atratores pullback para um mesmo processo $T(\cdot, \cdot)$ então, dupla aplicação de $(i v)$ nos daria $\mathcal{A}(t)=\mathcal{A}^{\prime}(t)$ para todo $t \in \mathbb{R}$.

Por último, demonstremos que a noção de atrator pullback é uma extensão da de atrator global ou, em termos formais, que um semigrupo $\{T(t): t \geq 0\}$ em $X$ possui atrator global $\mathcal{A}$ se, e somente se, o processo $\{T(t-s): t \geq s\}$, a ele associado, possui atrator pullback $\{\mathcal{A}(t): t \in \mathbb{R}\}$. E, nestas condições, temos $\mathcal{A}(t)=\mathcal{A}$ para todo $t \in \mathbb{R}$.

De fato, suponhamos que o semigrupo $\{T(t): t \geq 0\}$ possua atrator global $\mathcal{A}$ e definamos, para cada real $t$, o conjunto $\mathcal{A}(t):=\mathcal{A}$. Afirmamos que $\{\mathcal{A}(t): t \in \mathbb{R}\}$ é o atrator pullback para o processo $\{T(t-s): t \geq s\}$. Com efeito, a compacidade de cada $\mathcal{A}(t)$ segue da compacidade de $\mathcal{A}$ e, pela invariância de $\mathcal{A}$ por $T(\cdot)$, vemos que $T(t-s) \mathcal{A}(s)=T(t-s) \mathcal{A}=\mathcal{A}=\mathcal{A}(t)$ sempre que $t \geq s$. Também conclui-se, de forma óbvia, que para todo real $t$ e todo limitado $B$ vale

$$
\lim _{s \rightarrow-\infty} \operatorname{dist}(T(t-s) B, \mathcal{A}(t))=\lim _{\tau \rightarrow \infty} \operatorname{dist}(T(\tau) B, \mathcal{A})=0 .
$$

Agora, para ver satisfeita a condição $(i v)$ seja $\mathcal{B}:=\{\mathcal{B}(t): t \in \mathbb{R}\}$ uma família de subconjuntos fechados de $X$ tal que, para cada real $t, \mathcal{B}(t)$ atrai todos os limitados de $X$ em sentido pullback e no instante $t$, então, em particular, para cada $t, \mathcal{B}(t)$ atrai o 
conjunto limitado $\mathcal{A}$ que, por sua invariância por $T(\cdot)$, nos mostra que

$$
0=\lim _{s \rightarrow-\infty} \operatorname{dist}(T(t-s) \mathcal{A}, \mathcal{B}(t))=\lim _{s \rightarrow-\infty} \operatorname{dist}(\mathcal{A}, \mathcal{B}(t))=\operatorname{dist}(\mathcal{A}, \mathcal{B}(t))
$$

e, como sabemos, $\operatorname{dist}(\mathcal{A}, \mathcal{B}(t))=0$ implica $\mathcal{A} \subset \overline{\mathcal{B}(t)}=\mathcal{B}(t)$, ou seja, $\mathcal{A}(t)=\mathcal{A} \subset \mathcal{B}(t)$ para todo $t$.

Reciprocamente, suponhamos que $\{\mathcal{A}(t): t \in \mathbb{R}\}$ seja o atrator pullback para o processo $\{T(t-s): t \geq s\}$. Fixado um $t^{\prime} \in \mathbb{R}$ qualquer provemos que $\mathcal{A}^{\prime}:=\mathcal{A}\left(t^{\prime}\right)$ é o atrator global para $\{T(t): t \geq 0\}$. Com efeito, comecemos estudando a atração. Para isso, seja $B$ um subconjunto limitado de $X$, da atração pullback em $t^{\prime}$, resulta que

$$
\begin{gathered}
\lim _{\tau \rightarrow \infty} \operatorname{dist}\left(T(\tau) B, \mathcal{A}^{\prime}\right)=\lim _{\tau \rightarrow \infty} \operatorname{dist}\left(T\left(t^{\prime}-\left(t^{\prime}-\tau\right)\right) B, \mathcal{A}\left(t^{\prime}\right)\right)= \\
\lim _{s \rightarrow-\infty} \operatorname{dist}\left(T\left(t^{\prime}-s\right) B, \mathcal{A}\left(t^{\prime}\right)\right)=0 .
\end{gathered}
$$

Daí, vemos que $\{T(t): t \geq 0\}$ é dissipativo e assintoticamente compacto $^{1}$, donde podemos aplicar o Teorema 1.3.1 para concluir que $\{T(t): t \geq 0\}$ possui atrator global $\mathcal{A}$. Logo, de 7.1.1 e da invariância de $\mathcal{A}$, tem-se $\mathcal{A} \subset \mathcal{A}^{\prime}$ e, pelo que provamos no primeiro caso, conclui-se também que $\mathcal{A}^{\prime}=\mathcal{A}\left(t^{\prime}\right) \subset \mathcal{A}$, e nossa afirmação fica provada.

\subsection{Processos de evolução de tipo gradiente}

A partir de agora, nos restringiremos ao estudo dos processos de evolução de tipo gradiente (veja [8]), que é o principal objeto de estudo deste capítulo. Para introduzilos, devemos fazer mais algumas definições pertinentes. Comecemos pela noção de invariância isolada, que no caso não autônomo assume a seguinte forma:

Definição 7.2.1. Se $A=\{A(t): t \in \mathbb{R}\}$ é uma família invariante por $T(\cdot, \cdot)$, diz-se que A é uma família invariante isolada quando existe $\delta>0$ tal que se $\xi: \mathbb{R} \rightarrow X$ é uma solução global para $T(\cdot, \cdot) \operatorname{com} \xi(t) \in \mathcal{O}_{\delta}(A(t))$, para todo $t \in \mathbb{R}$, então $\xi(t) \in A(t)$ para todo real $t$.

Um conjunto finito $\mathbf{A}:=\left\{A_{1}, \cdots, A_{n}\right\}$ de famílias invariantes chama-se um conjunto finito de famílias invariantes isoladas quando cada família $A_{j}$ é uma família invariante isolada e existe $\delta>0$ tal que para todo $t \in \mathbb{R}$ tem-se $\mathcal{O}_{\delta}\left(A_{j}(t)\right) \cap$ $\mathcal{O}_{\delta}\left(A_{i}(t)\right)=\varnothing$ sempre que $i \neq j$.

\footnotetext{
${ }^{1}$ Com efeito, sejam $\left(t_{j}\right)_{j \in \mathbb{N}}$ uma sequência em $\mathbb{R}^{+}$com $t_{j} \rightarrow \infty$ e $\left(x_{j}\right)_{j \in \mathbb{N}}$ uma sequência limitada em $X$. Segue-se que o conjunto limitado $B:=\left\{x_{j}: j \in \mathbb{N}\right\}$ é atraído por $\mathcal{A}^{\prime}$, donde, em particular, $\lim _{j \rightarrow \infty} \operatorname{dist}\left(T\left(t_{j}\right) B, \mathcal{A}^{\prime}\right)=0$. Assim, para cada natural $k$ existe $j_{k}$ tal que $d\left(T\left(t_{j_{k}}\right) x_{j_{k}}, \mathcal{A}^{\prime}\right) \leq$ $\operatorname{dist}\left(T\left(t_{j_{k}}\right) B, \mathcal{A}^{\prime}\right)<\frac{1}{k}$ e, por isso, para cada $k$ existe $y_{k} \in \mathcal{A}^{\prime} \operatorname{com} d\left(T\left(t_{j_{k}}\right) x_{j_{k}}, y_{k}\right)<\frac{1}{k}$ e daí concluise, pela compacidade de $\mathcal{A}^{\prime}$, que a subsequência $\left(T\left(t_{j_{k}}\right) x_{j_{k}}\right)_{k \in \mathbb{N}}$ de $\left(T\left(t_{j}\right) x_{j}\right)_{j \in \mathbb{N}}$ possui subsequência convergente.
} 
No caso de semigrupos de tipo gradiente o conceito de estrutura homoclínica desempenha um papel fundamental, entretanto, ainda que para processos não autônomos tenhamos uma extensão deste conceito, a noção que se mostra mais eficiente é a de recorrência por cadeia, que a seguir definimos (Veja [12], para uma versão autônoma ligeiramente diferente mas que leva o mesmo nome, e [8] para a que aqui apresentamos):

Definição 7.2.2. Seja $\{T(t, s): t \geq s\}$ um processo de evolução em um espaço métrico $X$ possuindo atrator pullback $\mathcal{A}=\{\mathcal{A}(t): t \in \mathbb{R}\}$ e um conjunto finito de famílias invariantes isoladas $\mathbf{A}=\left\{A_{1}, \cdots, A_{n}\right\}$ tais que $A_{j}(t) \subset \mathcal{A}(t)$ para cada $t \in \mathbb{R}$ e cada $j=1, \cdots, n$.

Se $\delta>0$ é como na (segunda parte da) Definição 7.2.1, dadas uma família invariante $A \in \mathbf{A}$ e um $\varepsilon_{0} \in(0, \delta)$, para $\varepsilon \in\left(0, \varepsilon_{0}\right)$, uma $\varepsilon$-cadeia de A para $A$ (com $k \in \mathbb{N}$ "vértices") é uma coleção finita de indices $\left\{i_{1}, \cdots, i_{k}\right\} \subset\{1, \cdots, n\}$ juntamente com uma coleção de números reais $s_{j}, \tau_{j}, t_{j}$, tais que $s_{j} \leq \tau_{j} \leq t_{j}$ para $j=1,2, \cdots k$, e uma coleção de pontos $\left\{x_{j} \in X: j=1,2, \cdots, k\right\}$, satisfazendo

$$
\begin{gathered}
x_{j} \in \mathcal{O}_{\epsilon}\left(A_{i_{j}}\left(s_{j}\right)\right), T\left(\tau_{j}, s_{j}\right) x_{j} \notin \mathcal{O}_{\epsilon_{0}}\left(\bigcup_{i=1}^{n} A_{i}\left(\tau_{i}\right)\right) e \\
T\left(t_{j}, s_{j}\right) x_{j} \in \mathcal{O}_{\epsilon}\left(A_{i_{(j+1)}}\left(t_{i}\right)\right), \text { para } j=1,2, \cdots, k,
\end{gathered}
$$

onde $A_{i_{(k+1)}}:=A_{1}:=A$.

Uma família invariante $A \in \mathbf{A}$ chama-se recorrente por cadeia, quando existe $\varepsilon_{0} \in(0, \delta)$ e uma $\varepsilon$-cadeia de A para A para todo $\varepsilon \in\left(0, \varepsilon_{0}\right)$.

Observação 7.2.3. Observemos que a introdução do $\varepsilon_{0}$ na definição anterior é necessária simplesmente para cobrir o caso em que $k=1$, uma vez que, quando $k \geq 2$, é automaticamente verdade que a evolução de um ponto $x_{j}$ deve deixar a vizinhança $\mathcal{O}_{\epsilon_{0}}\left(\bigcup_{i=1}^{n} A_{i}(t)\right)$, para algum instante $t$, enquanto vai de uma vizinhança de uma "seção" de um conjunto invariante isolado para outra.

O conceito de sistema dinâmico de tipo gradiente já pode ser definido também para o caso não autônomo.

Definição 7.2.4. Seja $\{T(t, s): t \geq s\}$ um processo de evolução em um espaço métrico $X$, possuindo atrator pullback $\mathcal{A}=\{\mathcal{A}(t): t \in \mathbb{R}\}$ e um conjunto finito de famílias invariantes isoladas $\Xi=\left\{\Xi_{1}, \cdots, \Xi_{n}\right\}$ tais que $\Xi_{j}(t) \subset \mathcal{A}(t)$ para cada $t \in \mathbb{R}$ e cada $j=1, \cdots, n$.

Diz-se que $\{T(t, s): t \geq s\}$ é um processo de evolução de tipo gradiente com respeito $\grave{a} \Xi$ quando verifica as seguintes duas propriedades: 
(H1) Para toda solução global $\xi: \mathbb{R} \rightarrow X \operatorname{com} \xi(t) \in \mathcal{A}(t)$ para todo real $t$, existem familias invariantes $\Xi_{i}, \Xi_{j} \in \boldsymbol{\Xi}$ tais que

$$
\lim _{t \rightarrow-\infty} d\left(\xi(t), \Xi_{i}(t)\right)=0 \text { e } \lim _{t \rightarrow \infty} d\left(\xi(t), \Xi_{j}(t)\right)=0 .
$$

(H2) Não existe família invariante $\Xi \in \Xi$ que seja recorrente por cadeia.

Como mencionamos anteriormente, para o caso dos processos tem-se também a noção de estrutura homoclínica, que está fortemente relacionada ao conceito de recorrência por cadeia como definido acima (veja [8] para maiores detalhes).

Definição 7.2.5. Seja $\{T(t, s): t \geq s\}$ um processo de evolução num espaço métrico $X$ e $\boldsymbol{\Xi}=\left\{\Xi_{1}, \cdots, \Xi_{n}\right\}$ um conjunto finito de familias invariantes isoladas.

Uma estrutura homoclínica associada a $\boldsymbol{\Xi}$ é um subconjunto $\left\{\Xi_{i_{1}}, \cdots, \Xi_{i_{k}}\right\}$ de $\Xi$ em conjunto com uma coleção de soluções globais $\left\{\xi_{1}, \cdots, \xi_{k}\right\}$ tais que para $j=$ $1,2, \cdots, k$

$$
\lim _{t \rightarrow-\infty} d\left(\xi_{j}(t), \Xi_{i_{j}}(t)\right)=0 \text { e } \lim _{t \rightarrow \infty} d\left(\xi_{j}(t), \Xi_{i_{(j+1)}}(t)\right)=0
$$

onde $\Xi_{i_{(k+1)}}:=\Xi_{i_{1}}$.

Terminemos a seção apresentando a noção de conjuntos instáveis, que é bastante útil quando se deseja descrever o comportamento assintótico dos processos de evolução. Analogamente ao que fizemos para os semigrupos, é por meio deles que construímos nossos "conjuntos de Morse" também no caso dos processos não autônomos.

Definição 7.2.6. Se $\Xi=\{\Xi(t): t \in \mathbb{R}\}$ é uma família invariante para o processo $T(\cdot, \cdot)$, seu conjunto instável, denotado por $W^{u}(\Xi)$, é o conjunto dado

$$
\begin{gathered}
W^{u}(\Xi):=\{(t, x) \in \mathbb{R} \times X \text { : existe uma solução global } \xi: \mathbb{R} \rightarrow X \\
\left.\operatorname{com} \xi(t)=x e \lim _{s \rightarrow-\infty} d(\xi(s), \Xi(s))=0\right\} .
\end{gathered}
$$

Para cada real t, a seção do conjunto instável de $\Xi$ no instante t é o conjunto dado por

$$
W^{u}(\Xi)(t):=\left\{x \in X:(x, t) \in W^{u}(\Xi)\right\}
$$

\subsection{Decomposição de Morse não autônoma}

No caso dos semigrupos, a decomposição de Morse é o elemento fundamental na construção das funções de Lyapunov. Para definir este conceito, de forma consistente com o contexto autônomo, comecemos estendendo a noção de atrator local, que no caso não autônomo assume a seguinte forma: 
Definição 7.3.1. Seja $\{T(t, s): t \geq s\}$ um processo de evolução num espaço métrico $X$ possuindo atrator pullback $\{\mathcal{A}(t): t \in \mathbb{R}\}$. Diz-se que uma familia invariante isolada $A:=\{A(t): t \in \mathbb{R}\}$, com $A(t) \subset \mathcal{A}(t)$ para cada real $t$, é um atrator local (não autônomo) para $T(\cdot, \cdot)$ quando $W^{u}(A)(t)=A(t)$ para cada real $t$.

Se $A=\{A(t): t \in \mathbb{R}\}$ é um atrator local para $T(\cdot, \cdot)$, seu repulsor complementar é a família de subconjuntos $A^{*}:=\left\{A^{*}(t): t \in \mathbb{R}\right\}$, onde para cada real $t$

$$
A^{*}(t):=\{x \in \mathcal{A}(t): d(T(\tau, t) x, A(\tau)) \text { não converge a zero quando } \tau \rightarrow \infty\} .
$$

É óbvio que, para todo real $t, A(t) \cap A^{*}(t)=\varnothing$.

Provemos que esta última definição coincide com a Definição 4.1.1 no caso de processos autônomos, isto é, que se $\{T(t): t \geq 0\}$ é um semigrupo em $X$ possuindo atrator global $\mathcal{A}$, então um conjunto $A \subset \mathcal{A}$ é um atrator local no sentido da Definição 4.1.1 se, e somente se, a família constante $\{A(t): A(t):=A$ para todo $t \in \mathbb{R}\}$ é um atrator local para o processo $\{T(t-s): t \geq s\}$ segundo a Definição 7.3.1.

Com efeito, se $A \subset \mathcal{A}$ é um atrator local em sentido autônomo, é claro que a família $\{A(t): A(t):=A$ para todo $t \in \mathbb{R}\}$ é invariante com $A(t)=A \subset \mathcal{A}=\mathcal{A}(t)$ para todo real $t$ (recordando que, neste caso, o atrator pullback para $\{T(t-s): t \geq s\}$ é constante e dado por $\{\mathcal{A}(t): \mathcal{A}(t):=\mathcal{A}$ para todo $t \in \mathbb{R}\})$. Agora, é claro que, para cada $t$, temos $W^{u}(A)(t)=W^{u}(A)$ (onde o conjunto instável da direita deve ser entendido como no sentido do item (a) da Definição 2.2.3 e o da esquerda no sentido da Definição 7.2.6) e então, se $\varepsilon>0$ é tal que $\omega\left(\mathcal{O}_{\varepsilon}(A)\right)=A, \delta \in(0, \varepsilon)$ e $\delta^{\prime} \in(0, \delta)$ tais que

$$
\gamma^{+}\left(\mathcal{O}_{\delta^{\prime}}(A)\right) \subset \mathcal{O}_{\delta}(A)
$$

e $\xi: \mathbb{R} \rightarrow X$ uma solução global com $\lim _{t \rightarrow-\infty} d(\xi(t), A)=0$ segue-se que, para algum $t_{0} \in \mathbb{R}, d(\xi(t), A)<\delta^{\prime}$ sempre que $t \leq t_{0}$, donde conclui-se que $\xi(t)$ deverá pertencer a $\mathcal{O}_{\delta}(A)$ para todo real $t, \log 0 \gamma(\xi) \subset \omega\left(\mathcal{O}_{\varepsilon}(A)\right)=A$, mostrando que $W^{u}(A) \subset A$ e como a inclusão contrária é evidente da invariância de $A$, levando em conta a Proposição 1.1.2, conclui-se que $W^{u}(A)(t)=A=A(t)$ para todo $t$.

Por outro lado, o fato de que $\omega\left(\mathcal{O}_{\varepsilon}(A)\right)=A=A(t)$ para todo $t$, obriga a família $\{A(t): A(t):=A$ para todo $t \in \mathbb{R}\}$ a ser uma família isolada (isso se pode ver realizando-se um argumento semelhante ao feito acima) e assim conclui-se que $\{A(t)$ : $A(t):=A$ para todo $t \in \mathbb{R}\}$ é um atrator local não autônomo para $\{T(t-s): t \geq s\}$. Além disso, observemos que nestas condições, a família $\{A(t): A(t)=A, t \in \mathbb{R}\}$ não pode ser recorrente por cadeia, pois se assim o fosse, seria possível, por meio de argumentos habituais, construir uma solução global, não completamente dentro de $A$, que para trás convergiria para $A$, o que é absurdo porque o conjunto instável de $A$ é trivial. 
Reciprocamente, suponhamos que $\{A(t): A(t):=A$ para todo $t \in \mathbb{R}\}$ seja um atrator local para o processo $\{T(t-s): t \geq s\}$ no sentido da Definição 7.3.1.

Por um lado, pelo fato de que $\{A(t): t \in \mathbb{R}\}$ é invariante isolada em sentido não autônomo, conclui-se a existência de $\varepsilon>0$ tal que $A$ é o invariante maximal (em sentido autônomo) contido em $\mathcal{O}_{\varepsilon}(A)$, ou seja, segue-se que $A$ é invariante isolado em sentido autônomo (a invariância de $A$ conclui-se do fato de que $\{A(t): A(t):=A$ para todo $t \in \mathbb{R}\}$ é invariante pelo processo e é uma família constante).

Por outro, pelos fatos de que $W^{u}(A)(t)=W^{u}(A)$ e $A(t)=A$ para todo $t$, segue-se que $W^{u}(A)=A$, e daí conclui-se facilmente que, dado $\delta \in\left(0, \frac{\varepsilon}{2}\right)$ existe $\delta^{\prime} \in(0, \delta)$ de maneira que

$$
\gamma^{+}\left(\mathcal{O}_{\delta^{\prime}}(A)\right) \subset \mathcal{O}_{\delta}(A)
$$

donde $\omega\left(\mathcal{O}_{\delta^{\prime}}(A)\right) \subset \overline{\mathcal{O}_{\delta}(A)} \subset \mathcal{O}_{\varepsilon}(A)$ e então, pela maximalidade de $A$ em $\mathcal{O}_{\varepsilon}(A)$ obtém-se que $\omega\left(\mathcal{O}_{\delta^{\prime}}(A)\right) \subset A$ e a inclusão que falta resulta imediata da invariância de $A$, completando a prova da afirmação.

Vimos, no caso autônomo, que se $A$ é um atrator local para um dado semigrupo, então é quase imediato que seu repulsor complementar $A^{*}$ é um conjunto fechado de $X$. Contudo, no caso não autônomo a situação não é tão simples. Com relação a este fato, observemos apenas que se $A=\{A(t): t \in \mathbb{R}\}$ é um atrator local para o processo de evolução $\{T(t, s): t \geq s\}, A^{*}=\left\{A^{*}(t): t \in \mathbb{R}\right\}$ seu repulsor complementar e existe $\varepsilon>0$ tal que $\mathcal{A}(t) \cap \mathcal{O}_{\varepsilon}(A(t)) \cap \mathcal{O}_{\varepsilon}\left(A^{*}(t)\right)=\varnothing$ para todo real $t$, então $A^{*}(t)$ é um conjunto fechado para todo real $t$.

Com efeito, suponhamos que essa hipótese de separação se verifique e consideremos $\left(x_{n}\right)_{n \in \mathbb{N}}$ uma sequência de pontos de $X \operatorname{com} x_{n} \in A^{*}(s)$, para todo natural $n$ e um certo real $s$ fixado, tal que $x_{n} \rightarrow x \in \mathcal{A}(s) \backslash A^{*}(s)$, daí segue-se que $\lim _{t \rightarrow \infty} d(T(t, s) x, A(t))=0$ então, fixemos $t>s$ tal que $d(T(t, s) x, A(t))<\varepsilon$ e, pela continuidade da aplicação $T(t, s): X \rightarrow X, n_{0} \in \mathbb{N}$ tal que $d\left(T(t, s) x_{n_{0}}, A(t)\right)<\varepsilon$, mas, pela invariância de $A^{*}$ (confira a proposição abaixo) deveríamos ter $T(t, s) x_{n_{0}} \in A^{*}(t)$, contradizendo $\mathcal{A}(t) \cap \mathcal{O}_{\varepsilon}(A(t)) \cap \mathcal{O}_{\varepsilon}\left(A^{*}(t)\right)=\varnothing$.

Não obstante, podemos concluir facilmente a invariância do repulsor complementar $A^{*}$, como prova a seguinte proposição:

Proposição 7.3.2. Se $A=\{A(t): t \in \mathbb{R}\}$ é um atrator local para $\{T(t, s): t \geq s\}$, então seu repulsor complementar, $A^{*}=\left\{A^{*}(t): t \in \mathbb{R}\right\}$, é uma família invariante.

Demonstração: Com efeito, fixados $t \geq s$, dado $w \in A^{*}(s)$ se $T(t, s) w \notin A^{*}(t)$, por definição,

$$
\lim _{\tau \rightarrow \infty} d(T(\tau, t) T(t, s) w, A(\tau))=0
$$


isto é,

$$
\lim _{\tau \rightarrow \infty} d(T(\tau, s) w, A(\tau))=0
$$

dizendo que $w \notin A^{*}(s)$, o que é contrário à escolha de $w$. Este argumento nos diz que $T(t, s) A^{*}(s) \subset A^{*}(t)$.

Reciprocamente, se $z \in A^{*}(t) \subset \mathcal{A}(t)=T(t, s) \mathcal{A}(s)$, seja $w$ um ponto qualquer de $\mathcal{A}(s)$ tal que $z=T(t, s) w$, segue-se imediatamente que $w \in A^{*}(s)$, porque, caso contrário,

$$
\begin{gathered}
0=\lim _{\tau \rightarrow \infty} d(T(\tau, s) w, A(\tau))=\lim _{\tau \rightarrow \infty} d(T(\tau, t) T(t, s) w, A(\tau))= \\
\lim _{\tau \rightarrow \infty} d(T(\tau, t) z, A(\tau)),
\end{gathered}
$$

$\operatorname{logo} z \notin A^{*}(t)$, contradizendo sua escolha, mostrando que $T(t, s) A^{*}(s) \supset A^{*}(t)$ e completando a prova.

Como destacamos anteriormente, o Lema 4.1.2 foi decisivo na prova da continuidade da função de Lyapunov para os pares atrator-repulsor dos semigrupos. A seguir, apresentamos sua reformulação não autônoma restrita ao atrator pullback, que neste contexto não autônomo também desempenha papel de destaque.

Lema 7.3.3. Seja $A=\{A(t): t \in \mathbb{R}\}$ um atrator local não recorrente por cadeia para o processo de evolução $\{T(t, s): t \geq s\}$ possuindo atrator pullback $\mathcal{A}=\{\mathcal{A}(t): t \in \mathbb{R}\}$, tal que para algum $\varepsilon>0$

$$
\mathcal{A}(t) \cap \mathcal{O}_{\varepsilon}(A(t)) \cap \mathcal{O}_{\varepsilon}\left(A^{*}(t)\right)=\varnothing \text {, para todo real } t .
$$

Então, para cada $\delta \in(0, \varepsilon)$ existe $\delta^{\prime} \in(0, \delta)$ de modo que

$$
T(t, s)\left(\mathcal{A}(s) \cap \mathcal{O}_{\delta^{\prime}}(A(s))\right) \subset \mathcal{A}(t) \cap \mathcal{O}_{\delta}(A(t)), \text { toda vez que } t \geq s .
$$

Demonstração: Com efeito, caso contrário, poderíamos encontrar $\delta>0$, uma sequência $\left(x_{j}\right)_{j \in \mathbb{N}}$ de pontos de $X$ e sequências $\left(s_{j}\right)_{j \in \mathbb{N}}$ e $\left(\tau_{j}\right)_{j \in \mathbb{N}}$ de números reais, tais que

$$
\begin{gathered}
s_{j} \leq \tau_{j} \text { qualquer que seja } j \in \mathbb{N}, \\
x_{j} \in \mathcal{A}\left(s_{j}\right) \text { para todo natural } j, \\
d\left(x_{j}, A\left(s_{j}\right)\right)<\frac{1}{j} \text { e } d\left(T\left(\tau_{j}, s_{j}\right) x_{j}, A\left(\tau_{j}\right)\right) \geq \delta \text { também para todo } j .
\end{gathered}
$$

Então, do fato de que $d\left(x_{j}, A\left(s_{j}\right)\right)<\frac{1}{j}$ para todo $j$, poderíamos escolher $j_{0} \in \mathbb{N}$ com $d\left(x_{j}, A\left(s_{j}\right)\right)<\frac{1}{j}<\varepsilon$ para todo $j \geq j_{0}$, portanto, em particular, $x_{j} \notin A^{*}\left(s_{j}\right)$ sempre 
que $j \geq j_{0}$, donde seguiria a existência de uma sequência de números reais $\left(t_{j}\right)_{j \in \mathbb{N}}$ com $t_{j} \geq \tau_{j} \mathrm{e}$

$$
d\left(T\left(t_{j}, s_{j}\right) x_{j}, A\left(t_{j}\right)\right)<\frac{1}{j} \text { para todo } j \geq j_{0} .
$$

Mas esta condição não poderia se verificar, porque a mesma significa que $A$ é recorrente por cadeia, o que contrariaria as hipóteses.

Podemos demonstrar que a propriedade dada no último resultado é suficiente para caracterizar os atratores locais não recorrentes por cadeia. Mais precisamente, temos a seguinte proposição:

Proposição 7.3.4. Seja $A=\{A(t): t \in \mathbb{R}\}$ uma familia invariante isolada para o processo de evolução $\{T(t, s): t \geq s\}$, possuindo atrator pullback $\{\mathcal{A}(t): t \in \mathbb{R}\}$, tal que $A(t) \subset \mathcal{A}(t)$, para cada $t \in \mathbb{R}$.

Se a propriedade de que, para cada $\delta>0$ existe $\delta^{\prime} \in(0, \delta)$ com

$$
T(t, s)\left(\mathcal{A}(s) \cap \mathcal{O}_{\delta^{\prime}}(A(s))\right) \subset \mathcal{A}(t) \cap \mathcal{O}_{\delta}(A(t)), \text { toda vez que } t \geq s,
$$

se verifica, então $A=\{A(t): t \in \mathbb{R}\}$ é um atrator local não recorrente por cadeia para $\{T(t, s): t \geq s\}$.

Demonstração: Em primeiro lugar, por hipótese, $A$ é uma família invariante isolada.

Em segundo, pela invariância de $A, A(t) \subset W^{u}(A)(t)$ para cada real $t$, e se $\xi$ : $\mathbb{R} \rightarrow X$ é uma solução global de $T(\cdot, \cdot)$ com $\lim _{s \rightarrow-\infty} d(\xi(s), A(s))=0$, seja $\delta>0$ tal que a invariância maximal de $A$ se verifique nas vizinhanças $\mathcal{O}_{\delta}(A(t))$ e escolhamos $\delta^{\prime} \in(0, \delta)$ com

$$
T(t, s)\left(\mathcal{A}(s) \cap \mathcal{O}_{\delta^{\prime}}(A(s))\right) \subset \mathcal{A}(t) \cap \mathcal{O}_{\delta}(A(t)) \text {, sempre que } t \geq s .
$$

Agora, tomemos $s_{\delta^{\prime}} \in \mathbb{R}$ tal que $d(\xi(s), A(s))<\delta^{\prime}$ toda vez que $s \leq s_{\delta}, \log$ o $d(\xi(t), A(t))<$ $\delta$ para todo real $t$, ou seja, $\xi(t) \in \mathcal{O}_{\delta}(A(t))$ para todo real $t$ e, pela maximalidade de $A$, resulta que $\xi(t) \in A(t)$ para todo $t \in \mathbb{R}$, donde $W^{u}(A)(t) \subset A(t)$ para todo $t$, dizendo que $A$ é um atrator local.

Finalmente, sejam $\delta>0$, sequências de números reais $\left(s_{j}\right)_{j \in \mathbb{N}},\left(t_{j}\right)_{j \in \mathbb{N}}$ e $\left(\tau_{j}\right)_{j \in \mathbb{N}}$, uma sequência $\left(x_{j}\right)_{j \in \mathbb{N}}$ de pontos de $X$, tais que, para todo natural $j$

$$
\begin{gathered}
x_{j} \in \mathcal{A}\left(s_{j}\right), \\
s_{j} \leq t_{j} \leq \tau_{j}, \\
d\left(x_{j}, A\left(s_{j}\right)\right)<\frac{1}{j}, d\left(T\left(t_{j}, s_{j}\right) x_{j}, A\left(t_{j}\right)\right) \geq \delta \text { e } d\left(T\left(\tau_{j}, s_{j}\right) x_{j}, A\left(\tau_{j}\right)\right)<\frac{1}{j},
\end{gathered}
$$


mas, pelas hipóteses, podemos escolher $\delta^{\prime} \in(0, \delta)$ tal que

$$
T(t, s)\left(\mathcal{A}(s) \cap \mathcal{O}_{\delta^{\prime}}(A(s))\right) \subset \mathcal{A}(t) \cap \mathcal{O}_{\delta}(A(t)) \text {, tada vez que } t \geq s
$$

e além disso, escolher $j_{0} \in \mathbb{N}$ com $d\left(x_{j}, A\left(s_{j}\right)\right)<\frac{1}{j}<\delta^{\prime}$ sempre que $j \geq j_{0}$, logo

$$
d\left(T\left(t_{j}, s_{j}\right) x_{j}, A\left(t_{j}\right)\right) \geq \delta
$$

não pode ser verdade para $j \geq j_{0}$, o que nos diz que $A=\{A(t): t \in \mathbb{R}\}$ não pode ser recorrente por cadeia, completando a prova.

Para ilustrar o poder do Lema 7.3.3 provemos o seguinte.

Lema 7.3.5. Seja $A=\{A(t): t \in \mathbb{R}\}$ um atrator local para $\{T(t, s): t \geq s\}$ satisfazendo as hipóteses do Lema 7.3.3.

Se, para algum real $t, K \subset \mathcal{A}(t)$ é um conjunto compacto com $K \cap A^{*}(t)=\varnothing$, então

$$
\lim _{\tau \rightarrow \infty} \operatorname{dist}(T(\tau, t) K, A(\tau))=0
$$

Demonstração: Com efeito, caso contrário, existiriam sequências $\left(\tau_{j}\right)_{j \in \mathbb{N}}$, de números reais com $\tau_{j} \rightarrow \infty,\left(x_{j}\right)_{j \in \mathbb{N}}$ em $K, \operatorname{com} x_{j} \rightarrow x_{0} \in K$, e $\delta>0$ de maneira que

$$
d\left(T\left(\tau_{j}, t\right) x_{j}, A\left(\tau_{j}\right)\right) \geq \delta, \text { para todo natural } j .
$$

Então, pelo Lema 7.3.3, poderíamos escolher $\delta^{\prime} \in(0, \delta)$ tal que

$$
T(s, r)\left(\mathcal{A}(r) \cap \mathcal{O}_{\delta^{\prime}}(A(r))\right) \subset \mathcal{A}(s) \cap \mathcal{O}_{\delta}(A(s)) \text { sempre que } s \geq r .
$$

Assim, de (7.3.3) resultaria que

$$
d\left(T(s, t) x_{j}, A(s)\right) \geq \delta^{\prime} \text { para todo } s \in\left[t, \tau_{j}\right] \text { e todo natural } j .
$$

Pelo fato de que $\tau_{j} \rightarrow \infty$, usando (7.3.4), teríamos

$$
d\left(T(s, t) x_{0}, A(s)\right) \geq \delta^{\prime} \text { para todo } s \geq t
$$

o que nos diria que $x_{0} \in A^{*}(t)$, uma vez que $x_{0} \in K \subset \mathcal{A}(t)$, contradizendo $K \cap A^{*}(t)=$ $\varnothing$ e completando a prova.

Com as ferramentas que construímos até agora é possível definir, de forma precisa, o conceito de decomposição de Morse para processos. 
Definição 7.3.6. Seja $\{T(t, s): t \geq s\}$ um processo de evolução em $X$ possuindo atrator pullback $\{\mathcal{A}(t): t \in \mathbb{R}\}$.

Sejam também $A_{0}=\left\{A_{0}(t): t \in \mathbb{R}\right\}, A_{1}=\left\{A_{1}(t): t \in \mathbb{R}\right\}, \cdots, A_{n}=\left\{A_{n}(t): t \in\right.$ $\mathbb{R}\}, n+1$ atratores locais para o processo $\{T(t, s): t \geq s\}$ tais que, para cada real $t$, tem-se a cadeia crescente de inclusões

$$
\varnothing=A_{0}(t) \subset A_{1}(t) \subset \cdots \subset A_{n}(t)=\mathcal{A}(t) .
$$

Para cada real t e $j=1,2, \cdots, n$, seja

$$
M_{j}(t):=A_{j}(t) \cap A_{j-1}^{*}(t) .
$$

Nestas condiçôes, a n-upla ordenada $\mathbf{D}:=\left(M_{1}, M_{2}, \cdots, M_{n}\right)$ chama-se uma decomposição de Morse (não autônoma) para o atrator pullback $\{\mathcal{A}(t): t \in \mathbb{R}\}$ e cada conjunto $M_{j}(t)$ diz-se um conjunto de Morse (no instante $t$ ) desta decomposição.

Observemos que, se $A_{0}=\left\{A_{0}(t): t \in \mathbb{R}\right\}, A_{1}=\left\{A_{1}(t): t \in \mathbb{R}\right\}, \cdots, A_{n}=\left\{A_{n}(t)\right.$ : $t \in \mathbb{R}\}$ são $n+1$ atratores locais com

$$
\varnothing=A_{0}(t) \subset A_{1}(t) \subset \cdots \subset A_{n}(t)=\mathcal{A}(t),
$$

para cada real $t$, como no caso autônomo, simplesmente aplicando a definição de repulsor complementar, temos

$$
\varnothing=A_{n}^{*}(t) \subset A_{n-1}^{*}(t) \subset \cdots \subset A_{0}^{*}(t)=\mathcal{A}(t),
$$

também para todo $t$.

Descrevemos a seguir, inspirados na construção realizada para os semigrupos de tipo gradiente, a construção de uma decomposição de Morse para o atrator pullback de um processo de evolução de tipo gradiente apresentando a construção da coleção crescente de atratores locais, partindo da coleção de famílias invariantes isoladas do processo dado.

Para realizar esta tarefa o próximo resultado, que é a versão não autônoma do Lema 4.3.2, será de fundamental importância.

Lema 7.3.7. Seja $\{T(t, s): t \geq s\}$ um processo de evolução de tipo gradiente com respeito ao conjunto $\Xi=\left\{\Xi_{1}, \cdots, \Xi_{n}\right\}$ de familias invariantes isoladas. Então, para algum $i=1,2, \cdots, n, \Xi_{i}$ é um atrator local.

Demonstração: Em primeiro lugar, observemos que cada um dos $\Xi_{i}$ 's é uma família invariante isolada, simplesmente pela definição de processo de evolução de tipo gradiente, então devemos apenas demonstrar que existe algum $i=1,2, \cdots, n$ para o qual $W^{u}\left(\Xi_{i}\right)(t)=\Xi_{i}(t)$ para todo real $t$. 
De fato, porque caso contrário, para cada $i=1,2, \cdots, n$ existiria uma solução global $\xi_{i}: \mathbb{R} \rightarrow X \operatorname{com} \xi_{i}(t) \in \mathcal{A}(t)$ para todo real $t$ e $\xi_{i}\left(s_{i}\right) \notin \Xi_{i}\left(s_{i}\right)$ para algum real $s_{i}$ de maneira que $\lim _{t \rightarrow-\infty} d\left(\xi_{i}(t), \Xi_{i}(t)\right)=0$. Uma vez que quando $t \rightarrow \infty, \xi_{i}$ deve convergir para algum dos elementos de $\boldsymbol{\Xi}$, estes fatos produziriam uma estrutura homoclínica associada a $\boldsymbol{\Xi}$ e, portanto, uma família invariante $\Xi_{j} \in \boldsymbol{\Xi}$ recorrente por cadeia, o que não é possível.

Seja $\{T(t, s): t \geq s\}$ um processo de evolução de tipo gradiente com respeito ao conjunto finito de famílias invariantes isoladas $\boldsymbol{\Xi}=\left\{\Xi_{1}, \cdots, \Xi_{n}\right\}$.

Graças ao lema anterior, para algum $\sigma(1) \in I_{n}=\{1,2, \cdots, n\}, \Xi_{\sigma(1)} \in \Xi$ é um atrator local para $T(\cdot, \cdot)$. Seja $\Xi_{\sigma(1)}^{*}$ seu repulsor complementar como dado na Definição 7.3.1, então cada um dos conjuntos $\Xi_{i}(s)$ 's, com $i \neq \sigma(1)$ e $s \in \mathbb{R}$, está contido em $\Xi_{\sigma(1)}^{*}(s)$ e assim, para qualquer $z \in \mathcal{A}(t) \backslash\left(\Xi_{\sigma(1)}(t) \cup \Xi_{\sigma(1)}^{*}(t)\right)$ e qualquer que seja a solução global $\xi: \mathbb{R} \rightarrow X \operatorname{com} \xi(s) \in \mathcal{A}(s)$ para todo real $s$ e $\xi(t)=z$, temos

$$
\lim _{s \rightarrow-\infty} d\left(\xi(s), \Xi_{\sigma(1)}^{*}(s)\right)=0 \text { e } \lim _{s \rightarrow \infty} d\left(\xi(s), \Xi_{\sigma(1)}(s)\right)=0 .
$$

Por outro lado, podemos repetir o procedimento realizado na prova do último lema para concluir a existência de $\Xi_{\sigma(2)} \in \boldsymbol{\Xi}$, com $\sigma(2) \neq \sigma(1)$, tal que

$$
W^{u}\left(\Xi_{\sigma(2)}\right)(t) \cap \Xi_{\sigma(1)}^{*}(t)=\Xi_{\sigma(2)}(t) \text { qualquer que seja o real } t \text {. }
$$

Definamos, abusando um pouco da notação, para cada real $t$, o conjunto:

$\Xi_{\sigma(2)}^{*}(t):=\left\{x \in \Xi_{\sigma(1)}^{*}(t): d\left(T(\tau, t) x, \Xi_{\sigma(2)}(\tau)\right)\right.$ não converege para zero quando $\left.\tau \rightarrow \infty\right\}$.

Desta forma, para cada real $t$ e $i \notin\{\sigma(1), \sigma(2)\}$ temos $\Xi_{i}(t) \subset \Xi_{\sigma(2)}^{*}(t)$ e, analogamente ao que fizemos antes, conclui-se a existência de $\Xi_{\sigma(3)} \in \boldsymbol{\Xi}$, com $\sigma(3) \notin$ $\{\sigma(1), \sigma(2)\}$ tal que $W^{u}\left(\Xi_{\sigma(3)}\right)(t) \cap \Xi_{\sigma(2)}^{*}(t)=\Xi_{\sigma(3)}(t)$ para todo $t$ e definir $\Xi_{\sigma(3)}^{*}$ exatamente como em (7.3.5) acima, ou seja

$\Xi_{\sigma(3)}^{*}(t):=\left\{x \in \Xi_{\sigma(2)}^{*}(t): d\left(T(\tau, t) x, \Xi_{\sigma(3)}(\tau)\right)\right.$ não converege para zero quando $\left.\tau \rightarrow \infty\right\}$.

Continuando com este argumento até que todas as famílias invariantes sejam consideradas, obteremos, como no caso dos semigrupos, uma permutação do conjunto $I_{n}$, $\sigma: I_{n} \rightarrow I_{n}$, de forma que $\Xi_{\sigma(1)}$ é um atrator local para $\{T(t, s): t \geq s\}$ e para $i=2, \cdots, n$

$$
W^{u}\left(\Xi_{\sigma(i)}\right)(t) \cap \Xi_{\sigma(i-1)}^{*}(t)=\Xi_{\sigma(i)}(t) \text { para todo real } t,
$$

onde para $i=2, \cdots, n$

$\Xi_{\sigma(i)}^{*}(t):=\left\{x \in \Xi_{\sigma(i-1)}^{*}(t): d\left(T(\tau, t) x, \Xi_{\sigma(i)}(\tau)\right)\right.$ não converege para zero quando $\left.\tau \rightarrow \infty\right\}$. 
Ainda neste caso, por abuso de linguagem, diz-se que a família $\Xi_{\sigma(i)}^{*}=\left\{\Xi_{\sigma(i)}^{*}(t)\right.$ : $t \in \mathbb{R}\}$ é o repulsor associado a (ou complementar de) $\Xi_{\sigma(i)}$, para $i=2,3, \cdots, n$.

Levando em conta a construção acima podemos estabelecer o:

Lema 7.3.8. Nas condições da construção acima (ou seja, fixada uma permutação $\sigma: I_{n} \rightarrow I_{n}$ como acabamos de construir), se uma solução global $\xi: \mathbb{R} \rightarrow X$ é tal que

$$
\lim _{t \rightarrow-\infty} d\left(\xi(t), \Xi_{\sigma(i)}(t)\right)=0 \text { e } \lim _{t \rightarrow \infty} d\left(\xi(t), \Xi_{\sigma(j)}(t)\right)
$$

então $i \geq j$.

Demonstração: Com efeito, em primeiro lugar, observemos que se $A=\{A(t): t \in$ $\mathbb{R}\}$ é um atrator local para $\{T(t, s): t \geq s\}$ e $A^{*}=\left\{A^{*}(t): t \in \mathbb{R}\right\}$ seu repulsor complementar, então para toda solução global $\zeta: \mathbb{R} \rightarrow X \operatorname{com} \zeta(s) \in A^{*}(s)$, para algum real $s$, é fácil ver que $\zeta(t) \in A^{*}(t)$ para todo $t$ real.

Agora, observemos que o caso onde $j=1 \mathrm{em}$ (7.3.6) não ha nada o que fazer. Se $j=2$ e (7.3.6) se verifica então, pela definição de $\Xi_{\sigma(1)}^{*}$, temos $\xi(t) \in \Xi_{\sigma(1)}^{*}(t)$ para todo real $t$, logo, se $i<j=2$, ou seja, $i=1$, então $\xi(t) \in W^{u}\left(\Xi_{\sigma(1)}\right)(t)=\Xi_{\sigma(1)}(t)$ para todo $t$, o que contradiz $\Xi_{\sigma(1)}^{*}(t) \cap \Xi_{\sigma(1)}(t)=\varnothing$ para todo $t$, portanto $i$ deve ser maior do que ou igual a $j$.

Para ver o caso geral, suponhamos que $j \geq 3$ e que (7.3.6) se verifica. Neste caso não é difícil ver que $\xi(t) \in \Xi_{\sigma(j-1)}^{*}(t)$ para cada $t$ (para isso, veja a prova que fizemos quando provamos o resultado análogo para semigrupos de tipo gradiente no Capítulo 4) $\log$, se fosse $i<j$, pela definição dos repulsores, teríamos $\Xi_{\sigma(j-1)}^{*}(t) \subset \Xi_{\sigma(i-1)}^{*}(t)$ para todo $t$, donde $\xi(t) \in W^{u}\left(\Xi_{\sigma(i)}\right)(t) \cap \Xi_{\sigma(i-1)}^{*}(t)=\Xi_{\sigma(i)}(t)$ para todo $t$, assim, pelo fato de que as famílias em $\boldsymbol{\Xi}$ são isoladas, deveriamos ter $i=j$, e com esta contradição o lema está provado.

Provamos a seguir que, fixando uma permutação $\sigma: I_{n} \rightarrow I_{n}$, obtida por meio do procedimento que explicamos linhas acima, o conjunto $\left\{\Xi_{1}, \cdots, \Xi_{n}\right\}$, sob hipóteses bastante razoáveis, dá lugar a decomposição de Morse $\mathbf{D}:=\left(\Xi_{\sigma(1)}, \Xi_{\sigma(2)}, \cdots, \Xi_{\sigma(n)}\right)$ do atrator pullback $\{\mathcal{A}(t): t \in \mathbb{R}\}$, escolhendo-se convenientemente a sequência $A_{0}(t) \subset$ $A_{1}(t) \subset A_{2}(t) \subset \ldots \subset A_{n}(t)$ de atratores locais:

Com efeito, imitando a construção do caso autônomo, definimos para cada $t \in \mathbb{R}$, $A_{0}(t):=\varnothing, A_{1}(t):=\Xi_{\sigma(1)}(t)$ e para $j=2,3, \cdots, n$

$$
A_{j}(t):=A_{j-1}(t) \cup W^{u}\left(\Xi_{\sigma(j)}\right)(t)=\bigcup_{i=1}^{j} W^{u}\left(\Xi_{\sigma(i)}\right)(t) .
$$


É claro, do fato de que $T(\cdot, \cdot)$ é de tipo gradiente com respeito à família $\boldsymbol{\Xi}$, que $A_{n}(t)=$ $\mathcal{A}(t)$ para todo $t$ e podemos provar o seguinte importante resultado:

Teorema 7.3.9. Sejam $\{T(t, s): t \geq s\}$ um processo de evolução de tipo gradiente com respeito ao conjunto de famílias invariantes isoladas $\boldsymbol{\Xi}=\left\{\Xi_{1}, \cdots, \Xi_{n}\right\}$ e $\sigma: I_{n} \rightarrow I_{n}$ uma permutação obtida por meio do procedimento que explicamos acima.

Se existir um número real positivo $\delta$ tal que para todo $t \in \mathbb{R}$ e todo $j=1,2, \cdots, n-1$

$$
\mathcal{O}_{\delta}\left(A_{j}(t)\right) \cap\left(\bigcup_{i=j+1}^{n} \Xi_{\sigma(i)}(t)\right)=\varnothing,
$$

então, para cada $j=0,1, \cdots, n$, a família $A_{j}:=\left\{A_{j}(t): t \in \mathbb{R}\right\}$, com $A_{j}(t)$ definido em (7.3.7), é um atrator local para $\{T(t, s): t \geq s\}$ e, além disso, tem-se

$$
\Xi_{\sigma(j)}(t)=A_{j}(t) \cap A_{j-1}^{*}(t), \text { para todo real } t \text { e } j=1, \cdots, n .
$$

Consequentemente, $\mathbf{D}=\left(\Xi_{\sigma(1)}, \Xi_{\sigma(2)}, \cdots, \Xi_{\sigma(n)}\right)$ é uma decomposição de Morse para o atrator pullback $\{\mathcal{A}(t): t \in \mathbb{R}\}$.

Demonstração: Com efeito, é claro da definição que cada $A_{j}$ é uma família invariante e então, para todo $j$ e todo $t, A_{j}(t) \subset W^{u}\left(A_{j}\right)(t)$.

Por outro lado, se $x \in W^{u}\left(A_{j}\right)(t)$, existe uma solução global $\xi: \mathbb{R} \rightarrow X$ com $\xi(t)=x$ e $\lim _{s \rightarrow-\infty} d\left(\xi(s), A_{j}(s)\right)=0$ e, como $\{T(t, s): t \geq s\}$ é um processo de tipo gradiente, existe $k=1,2, \cdots, n$ de maneira que $\lim _{s \rightarrow-\infty} d\left(\xi(s), \Xi_{\sigma(k)}(s)\right)=0$. Mas, graças a (7.3.8), tem-se $k \leq j$, e assim $x \in W^{u}\left(\Xi_{\sigma(k)}\right)(t)$ com $k \leq j$, portanto $x \in A_{j}(t)$ e daí conclui-se que $W^{u}\left(A_{j}\right)(t) \subset A_{j}(t)$. Unindo isso ao que observamos acima, resulta que $W^{u}\left(A_{j}\right)(t)=A_{j}(t)$ para todo real $t$.

Agora, seja $\delta>0$ como em (7.3.8). Se $\xi: \mathbb{R} \rightarrow X$ é uma solução global tal que $\xi(t) \in \mathcal{O}_{\delta}\left(A_{j}(t)\right)$ para todo real $t$, segue-se, em particular, que $\lim _{s \rightarrow-\infty} d\left(\xi(s), \Xi_{\sigma(k)}(s)\right)=0$ para algum $k \leq j$, então $\xi(t) \in W^{u}\left(\Xi_{\sigma(k)}\right)(t) \subset A_{j}(t)$ para cada $t$, por isso $A_{j}$ é uma família invariante isolada para cada $j$.

Nestas condições, acabamos de provar que $A_{j}$ é um atrator local para o processo $\{T(t, s): t \geq s\}$ para cada $j=1,2, \cdots, n$.

Para provar a igualdade em (7.3.9), observemos, por um lado, que $\Xi_{\sigma(j)}(t) \subset A_{j}(t) \cap$ $A_{j-1}^{*}(t)$, para todo número real $t$ e $j=1,2, \cdots, n$, pois $\Xi_{\sigma(j)}(t) \subset A_{j}(t)$, simplesmente pela definição dos $A_{j}$ 's e se $x \in \Xi_{\sigma(j)}(t)$, qualquer que seja a solução $\xi: \mathbb{R} \rightarrow X$ com $\xi(t)=x$ tem-se $\xi(s) \in \Xi_{\sigma(j)}(s)$ toda vez que $s \geq t$ e assim (também graças a (7.3.8)) $\lim _{s \rightarrow \infty} d\left(\xi(s), A_{j-1}(s)\right)$ não pode ser zero, donde obtém-se $x \in A_{j-1}^{*}(t)$.

Por outro lado, dado $x \in A_{j}(t) \cap A_{j-1}^{*}(t)$ existe uma solução global $\xi: \mathbb{R} \rightarrow X$ com $\xi(t)=x$ de modo que $\lim _{s \rightarrow-\infty} d\left(\xi(s), \Xi_{\sigma(k)}(s)\right)$ para algum $k \leq j$. Como $\{T(t, s): t \geq s\}$ 
é de tipo gradiente, existe $i=1,2, \cdots, n$ tal que $\lim _{s \rightarrow \infty} d\left(\xi(s), \Xi_{\sigma(i)}(s)\right)$, mas do fato de que $x \in A_{j-1}^{*}(t)$ obtém-se que $\lim _{s \rightarrow \infty} d\left(\xi(s), A_{j-1}(s)\right)$ não é zero, então $i$ não pode ser menor do que ou igual a $j-1$, isto é, $i \geq j$, logo pela maneira com a qual a permutação $\sigma: I_{n} \rightarrow I_{n}$ foi construída, a única maneira destes dois fatos ocorrerem simultaneamente é quando $k=i=j$, e então, como $\xi$ não pode ser uma solução homoclínica, devemos ter $\xi(s) \in \Xi_{\sigma(j)}(s)$ para todo $s$, em particular, $x=\xi(t) \in \Xi_{\sigma(j)}(t)$ e a inclusão $A_{j}(t) \cap A_{j-1}^{*}(t) \subset \Xi_{\sigma(j)}(t)$ é também verdadeira, comprovando a igualdade $A_{j}(t) \cap A_{j-1}^{*}(t)=\Xi_{\sigma(j)}(t)$ e terminando a prova do teorema.

\subsection{Função de Lyapunov para um processo de evolução de tipo gradiente}

Utilizando as ferramentas construídas na seção anterior estabelecemos a existência de uma função de Lyapunov para os processos de evolução de tipo gradiente (veja a Definição 2.1.3 para o caso dos semigrupos).

As definições de função de Lyapunov não autônoma e de processo de evolução gradiente são dadas a seguir.

Definição 7.4.1. Diz-se que um processo de evolução $\{T(t, s): t \geq s\}$ possuindo atrator pullback $\{\mathcal{A}(t): t \in \mathbb{R}\}$ e conjunto finito $\boldsymbol{\Xi}=\left\{\Xi_{1}, \cdots, \Xi_{n}\right\}$ de familias invariantes isoladas com $\Xi_{j}(t) \subset \mathcal{A}(t)$ para todo $t \in \mathbb{R}$ e todo $j=1,2, \cdots, n$, é um processo de evolução gradiente com respeito $\boldsymbol{a} \Xi$ quando existe uma função $V: \mathbb{R} \times X \rightarrow \mathbb{R}$ satisfazendo as seguintes quatro propriedades:

(i) Para cada ponto $x \in X$ e cada $t \in \mathbb{R}$ a função real de uma variável real $[0, \infty) \ni$ $r \mapsto V(r+t, T(r+t, t) x) \in \mathbb{R}$ é não crescente.

(ii) Dados $t \in \mathbb{R}$ e $x \in \mathcal{A}(t), V(r+t, T(r+t, t) x)=V(t, x)$ para todo $r \geq 0$ se, e somente se, $x \in \bigcup_{i=1}^{n} \Xi_{i}(t)$.

(iii) Para cada $t \in \mathbb{R}$, a função real $V_{t}: \mathcal{A}(t) \rightarrow \mathbb{R}$, dada por $V_{t}(x):=V(t, x)$ para $x \in \mathcal{A}(t)$, é contínua (em $\mathcal{A}(t))$.

(iv) Para todo $i=1,2, \cdots, n, V$ é constante sobre o conjunto $\{(t, x) \in \mathbb{R} \times X: x \in$ $\left.\Xi_{i}(t)\right\}$.

Uma função $V: \mathbb{R} \times X \rightarrow \mathbb{R}$ satisfazendo as condições acima chama-se uma função de Lyapunov para o processo gradiente $\{T(t, s): t \geq s\}$ com respeito ao conjunto $\Xi$. 
Devemos observar que a definição de função de Lyapunov não autônoma, dada acima, estende a noção correspondente do caso autônomo no seguinte sentido:

Se $W: X \rightarrow \mathbb{R}$ é uma função de Lyapunov para um semigrupo $S(\cdot)$ possuindo atrator global $\mathcal{A}$ e com respeito à família finita disjunta $\mathbf{E}=\left\{E_{1}, \cdots, E_{n}\right\}$ de conjuntos invariantes isolados (veja a Definição 2.1.3) então, como é fácil ver, a função $V$ : $\mathbb{R} \times X \rightarrow \mathbb{R}$ dada por

$$
V(t, x):=W(x),(t, x) \in \mathbb{R} \times X
$$

é uma função de Lyapunov, no sentido da definição acima, para o processo $\{T(t, s)$ : $T(t, s):=S(t-s), t \geq s\}$ com respeito ao conjunto finito $\Xi=\left\{\Xi_{1}, \cdots, \Xi_{n}\right\}$ de famílias invariantes isoladas, onde $\Xi_{i}(t):=E_{i}$ para todo real $t$ e $i=1,2, \cdots, n$.

$\mathrm{Na}$ prova do próximo lema, que estabelece um tipo de continuidade das famílias invariantes $\left(A, A^{*}\right)$, seguindo as idéias encontradas em [10], utilizamos um resultado cuja demonstração será omitida, trata-se do Lema 2.10 en [10] e cuja demonstração é semelhante a prova do Lema 3.2.3. A leitura de sua demonstração pode ser omitida sem riscos ao entendimento dos demais resultados deste capítulo.

Lema 7.4.2. Seja $\{T(t, s): t \geq s\}$ um processo de evolução em um espaço métrico $X$ possuindo atrator pullback $\{\mathcal{A}(t): t \in \mathbb{R}\}$ e $\left(A, A^{*}\right)$ um par atrator-repulsor.

Então, para cada $t_{0} \in \mathbb{R}$ fixo

$$
\lim _{t \rightarrow t_{0}} d_{H}\left(A(t), A\left(t_{0}\right)\right)=0 \text { e } \lim _{t \rightarrow t_{0}} d_{H}\left(A^{*}(t), A^{*}\left(t_{0}\right)\right)=0 .
$$

Demonstração: Façamos somente a prova de que $\lim _{t \rightarrow t_{0}} d_{H}\left(A(t), A\left(t_{0}\right)\right)=0$, uma vez que a correspondente propriedade para o repulsor faz-se de modo semelhante.

Comecemos provando a semicontinuidade superior quando $t$ tende a $t_{0}$, isto é,

$$
\lim _{t \rightarrow t_{0}} \operatorname{dist}\left(A(t), A\left(t_{0}\right)\right)=0
$$

Com efeito, se isto não ocorre, existem $\varepsilon>0$ e uma sequência de números reais $\left(t_{j}\right)_{j \in \mathbb{N}} \operatorname{com} t_{j} \underset{j \rightarrow \infty}{\rightarrow} t_{0}$ tais que

$$
\operatorname{dist}\left(A\left(t_{j}\right), A\left(t_{0}\right)\right)>\varepsilon \text { para todo natural } j \text {. }
$$

Donde conclui-se, simplesmente pela definição da semidistância de Hausdorff, a existência de uma sequência $\left(x_{j}\right)_{j \in \mathbb{N}}$ de pontos de $X$ com $x_{j} \in A\left(t_{j}\right)$ e

$$
d\left(x_{j}, A\left(t_{0}\right)\right) \geq \varepsilon \text { para todo natural } j .
$$

Por outro lado, pela invariância da família $\{A(t): t \in \mathbb{R}\}$, para cada natural $j$, podemos escolher uma solução global $\xi_{j}: \mathbb{R} \rightarrow X$ do processo $T(\cdot, \cdot)$ tal que $\xi_{j}\left(t_{j}\right)=x_{j}$ 
e $\xi_{j}(t) \in A(t)$ para todo real $t$ e não é difícil ver (confira o Lema 7.5.3 na próxima seção) que a sequência $\left(\xi_{j}\right)_{j \in \mathbb{N}}$ possui uma subsequência, que indicaremos com a mesma notação, e existe uma solução global $\xi: \mathbb{R} \rightarrow X$ do processo $T(\cdot, \cdot)$ tal que $\lim _{j \rightarrow \infty} \xi_{j}(t)=$ $\xi(t)$ uniformemente para $t$ em compactos da reta. Daí conclui-se que

$$
d\left(x_{j}, \xi_{j}\left(t_{0}\right)\right) \leq d\left(\xi_{j}\left(t_{j}\right), \xi\left(t_{j}\right)\right)+d\left(\xi\left(t_{j}\right), \xi\left(t_{0}\right)\right)+d\left(\xi\left(t_{0}\right), \xi_{j}\left(t_{0}\right)\right) \underset{j \rightarrow \infty}{\rightarrow} 0 .
$$

Mas, $d\left(x_{j}, \xi_{j}\left(t_{0}\right)\right) \geq \inf _{x \in A\left(t_{0}\right)} d\left(x_{j}, x\right)=d\left(x_{j}, A\left(t_{0}\right)\right) \geq \varepsilon$ para todo natural $j$, contradizendo a convergência acima e estabelecendo (7.4.11).

De forma inteiramente análoga demonstra-se a semicontinuidade inferior, ou seja, que

$$
\lim _{t \rightarrow t_{0}} \operatorname{dist}\left(A\left(t_{0}\right), A(t)\right)=0
$$

completando a prova.

Com a ajuda do último lema podemos provar o principal resultado deste capítulo, o qual constitui uma versão não autônoma da Proposição 4.2.2, provada para semigrupos.

Proposição 7.4.3. Seja $\left(A, A^{*}\right)$ um par atrator-repulsor para um processo de evolução $\{T(t, s): t \geq s\}$ num espaço métrico $X$ possuindo atrator pullback $\{\mathcal{A}(t): t \in \mathbb{R}\}$.

Suponhamos que exista $\varepsilon_{0}>0$ com

$$
\mathcal{O}_{\varepsilon_{0}}(A(t)) \cap \mathcal{O}_{\varepsilon_{0}}\left(A^{*}(t)\right)=\varnothing \text {, para todo real } t
$$

e que o atrator local $A=\{A(t): t \in \mathbb{R}\}$ nãa seja recorrente por cadeia.

Então, existe uma função $k: \mathbb{R} \times X \rightarrow \mathbb{R}$ satisfazendo as seguintes quatro propriedades:

(i) Para cada ponto $x \in X$ e cada real $t \in \mathbb{R}$ a função real $[0, \infty) \ni r \mapsto k(r+$ $t, T(r+t, t) x) \in \mathbb{R}$ é não crescente.

(ii) Se, para cada real $t, k_{t}: X \rightarrow \mathbb{R}$ é a função dada por $k_{t}(x):=k(t, x)$, tem-se $k_{t}^{-1}(0)=A(t)$ e $k_{t}^{-1}(1) \cap \mathcal{A}(t)=A^{*}(t)$.

(iii) Dados $t \in \mathbb{R}$ e $x \in \mathcal{A}(t)$, se $k(r+t, T(r+t, t) x)=k(t, x)$ para todo $r \geq 0$, então $x \in A(t) \cup A^{*}(t)$.

(iv) $\left.k_{t}\right|_{\mathcal{A}(t)}: \mathcal{A}(t) \rightarrow \mathbb{R}$ é contínua em $\mathcal{A}(t)$, para cada $t \in \mathbb{R}$.

Demonstração: Em primeiro lugar, fazendo a convenção $d(x, \varnothing)=1$, associado ao $\operatorname{par}\left(A, A^{*}\right)$ definamos a função $l: \mathbb{R} \times X \rightarrow[0,1]$ por

$$
l(t, x):=\frac{d(x, A(t))}{d(x, A(t))+d\left(x, A^{*}(t)\right)}, \text { para }(t, x) \in \mathbb{R} \times X .
$$


Levando em conta (7.4.12) segue-se que $l$ está bem definida com $l(\mathbb{R} \times X) \subset[0,1]$. Provemos que ela é contínua em ambas as duas variáveis $(t, x)$ e, para cada real $t, l_{t}$ é uniformemente contínua em $X$ independentemente de $t$ (em outras palavras, que a família de funções $\left\{l_{t}: X \rightarrow[0,1]: t \in \mathbb{R}\right\}$ é uniformemente equicontínua onde, para cada real $t, l_{t}: X \rightarrow \mathbb{R}$ está dada por $\left.l_{t}(x):=l(t, x), x \in X\right)$.

Com efeito, graças a (7.4.12), tem-se

$$
d_{0}:=\inf _{t \in \mathbb{R}}\left\{\inf \left\{d(x, y): x \in A(t), y \in A^{*}(t)\right\}\right\} \geq \varepsilon_{0}>0,
$$

donde segue-se facilmente, assim como foi feito na prova da Proposição 4.2.2, que $|l(t, x)-l(t, y)| \leq \frac{2}{d_{0}} d(x, y)$, quaisquer que sejam os pontos $x$ e $y$ em $X$ e $t$ real.

Agora, para $(t, x)$ e $\left(t_{0}, x_{0}\right)$ em $\mathbb{R} \times X$ temos

$$
\begin{gathered}
\left|l(t, x)-l\left(t_{0}, x_{0}\right)\right| \leq \\
\frac{d_{H}\left(A(t), A\left(t_{0}\right)\right) d\left(x_{0}, A^{*}\left(t_{0}\right)\right)+d_{H}\left(A^{*}(t), A^{*}\left(t_{0}\right)\right) d\left(x_{0}, A\left(t_{0}\right)\right)}{\left[d(x, A(t))+d\left(x, A^{*}(t)\right)\right]\left[d\left(x_{0}, A\left(t_{0}\right)\right)+d\left(x_{0}, A^{*}\left(t_{0}\right)\right)\right]}+ \\
\frac{\left|d\left(x, A\left(t_{0}\right)\right)-d\left(x_{0}, A\left(t_{0}\right)\right)\right| d\left(x_{0}, A^{*}\left(t_{0}\right)\right)+\left|d\left(x, A^{*}\left(t_{0}\right)\right)-d\left(x_{0}, A^{*}\left(t_{0}\right)\right)\right| d\left(x_{0}, A\left(t_{0}\right)\right)}{\left[d(x, A(t))+d\left(x, A^{*}(t)\right)\right]\left[d\left(x, A\left(t_{0}\right)\right)+d\left(x_{0}, A^{*}\left(t_{0}\right)\right)\right]}
\end{gathered}
$$

donde, em virtude do Lema 7.4.2 e da continuidade da função distância de um ponto a um conjunto, obtém-se a continuidade da função $l: \mathbb{R} \times X \rightarrow[0,1]$ no ponto $\left(t_{0}, x_{0}\right)$.

Além disso, como se vê facilmente, $l_{t}^{-1}(0)=A(t)$ e $l_{t}^{-1}(1)=A^{*}(t)$.

A função satisfazendo as propriedades buscadas será a função $k: \mathbb{R} \times X \rightarrow \mathbb{R}$ dada por

$$
k(t, x):=\sup _{r \geq 0} l(r+t, T(r+t, t) x),(t, x) \in \mathbb{R} \times X .
$$

Mostremos que, efetivamente, a função $k: \mathbb{R} \times X \rightarrow \mathbb{R}$ possui as quatro propriedades enunciadas na proposição.

Observemos que, $k(\mathbb{R} \times X) \subset[0,1]$ pois $l(r+t, T(r+t, t) x) \in[0,1]$ para todo $x \in X$, $t \in \mathbb{R}$ e $r \geq 0$.

Para vermos que dados $t \in \mathbb{R}$ e $x \in X$, a função $[0, \infty) \ni r \mapsto k(r+t, T(r+t, t) x) \in$ $[0,1]$ é não crescente basta observarmos que, se $0 \leq r_{1} \leq r_{2}$ temos

$$
\begin{aligned}
& k\left(r_{2}+t, T\left(r_{2}+t, t\right) x\right)=\sup _{r \geq 0} l\left(r+r_{2}+t, T\left(r+r_{2}+t, t\right) x\right) \\
& =\sup _{r \geq r_{2}} l(r+t, T(r+t, t) x) \\
& \leq \sup _{r \geq r_{1}} l(r+t, T(r+t, t) x) \\
& =\sup _{r \geq 0} l\left(r+r_{1}+t, T\left(r+r_{1}+t, t\right) x\right)=k\left(r_{1}+t, T\left(r_{1}+t, t\right) x\right) .
\end{aligned}
$$

Está claro da definição de $k$ e da invariância de $A$ e $A^{*}$ que $k_{t}(A(t))=\{0\}$ e $k_{t}\left(A^{*}(t)\right)=\{1\}$. Agora, se $x \in X$ é tal que $k(t, x)=0$, então $l(r+t, T(r+t, t) x)=0$ 
para todos os $r \geq 0$. Em particular, $0=l(t, x)$, e assim, $x \in A(t)$, donde conclui-se que, $k_{t}^{-1}(0) \subset A(t)$ o que nos mostra a igualdade $k_{t}^{-1}(0)=A(t)$. Por outro lado, se $x \in \mathcal{A}(t)$ fosse tal que $k(x)=1$ mas $x \notin A^{*}(t)$, teríamos que $\lim _{r \rightarrow \infty} d(T(r+t, t) x, A(r+t))=0$. Da definição de $l$ obteríamos que $\lim _{r \rightarrow \infty} l(r+t, T(r+t, t) x)=0$. Então, existiria $r_{0}>0$ de modo que $1=k(t, x)=\sup _{0 \leq r \leq r} l(r+t, T(r+t, t) x)$. A continuidade da função real $[0, \infty) \ni r \mapsto l(r+t, T(r+t, t) x) \in[0,1]$ implicaria, por sua vez, a existência de $r^{\prime} \in\left[0, r_{0}\right]$ tal que $l\left(r^{\prime}+t, T\left(r^{\prime}+t, t\right) x\right)=1$, consequentemente $T\left(r^{\prime}+t, t\right) x \in$ $A^{*}\left(r^{\prime}+t\right)$ o que contrariaria $\lim _{r \rightarrow \infty} l(T(r+t, t) x, A(r+t))=0$, e assim, se $k(t, x)=1$ para algum $t \in \mathbb{R}$ e algum $x \stackrel{r \rightarrow \infty}{\in} \mathcal{A}(t)$ necessariamente $x \in A^{*}(t)$. Deste fato conclui-se que $k_{t}^{-1}(1) \cap \mathcal{A}(t) \subset A^{*}(t)$ e então que $k_{t}^{-1}(1) \cap \mathcal{A}(t)=A^{*}(t)$.

Provemos agora que, se $x \in \mathcal{A}(t)$ e $k(r+t, T(r+t, t) x)=k(t, x)$ para todo $r \geq 0$ então $x \in A(t) \cup A^{*}(t)$. Se $x \notin A(t) \cup A^{*}(t)$, então $\lim _{r \rightarrow \infty} d(T(r+t, t) x, A(r+t))=0$ e da definição de $k$ temos $k(t, x)=\lim _{r \rightarrow \infty} k(r+t, T(r+t, t) x)=0$. Dado que $k_{t}^{-1}(0)=A(t)$, $x$ deveria pertencer a $A(t)$ o que contradiz sua escolha.

Neste ponto, fixemos um número real $t$ e provemos a continuidade da função $k_{t}$ : $\mathcal{A}(t) \rightarrow \mathbb{R}$ em $\mathcal{A}(t)$

Como o que ocorreu no caso autônomo, aqui também dividimos a prova em três casos:

Caso 1) Continuidade de $k_{t}: \mathcal{A}(t) \rightarrow \mathbb{R}$ nos pontos de $A^{*}(t)$.

Uma vez que $l(t, x) \leq k(t, x) \leq 1$, para todo $t \in \mathbb{R}$ e todo $x \in X$, dados $x_{0} \in A^{*}(t)$ e $x \in X$ temos

$$
\left|k(t, x)-k\left(t, x_{0}\right)\right|=1-k(t, x) \leq 1-l(t, x),
$$

e isto em conjunto com a continuidade de $l: \mathbb{R} \times X \rightarrow \mathbb{R}$ em $\left(t, x_{0}\right)$ garantem a continuidade de $k_{t}: \mathcal{A}(t) \rightarrow \mathbb{R}$ em $x_{0}$.

Caso 2) Continuidade de $\left.k_{t}\right|_{\mathcal{A}(t)}: \mathcal{A}(t) \rightarrow \mathbb{R}$ nos pontos de $A(t)$.

Da equicontinuidade da família de funções $\left\{l_{t}: X \rightarrow \mathbb{R}: t \in \mathbb{R}\right\}$ (mais precisamente o fato de que $|l(t, x)-l(t, y)| \leq \frac{2}{d_{0}} d(x, y)$, quaisquer que sejam os pontos $x$ e $y$ em $X$ e $t$ real), dado $\varepsilon>0$, existe $\delta>0$ tal que $l_{s}\left(\mathcal{O}_{\delta}(A(s))\right) \subset[0, \varepsilon)$ para todo real $s$. Agora, o Lema 7.3.3 garante a existência de $\delta^{\prime} \in(0, \delta)$ tal que $T(r+t, t)\left(\mathcal{A}(t) \cap \mathcal{O}_{\delta^{\prime}}(A(t))\right) \subset$ $\mathcal{A}(r+t) \cap \mathcal{O}_{\delta}(A(r+t))$, sempre que $r \geq 0$ donde conlui-se que $l(r+t, T(r+t, t) x) \in[0, \varepsilon)$ para todo real $t$ sempre que $r \geq 0$ e $x \in \mathcal{A}(t) \cap \mathcal{O}_{\delta^{\prime}}(A(t))$, daí $k_{t}\left(\mathcal{A}(t) \cap \mathcal{O}_{\delta^{\prime}}(A(t))\right) \subset$ $[0, \varepsilon]$, o que nos proporciona a continuidade de $\left.k_{t}\right|_{\mathcal{A}(t)}: \mathcal{A}(t) \rightarrow \mathbb{R}$ nos pontos de $A(t)$.

Caso 3) Continuidade de $\left.k_{t}\right|_{\mathcal{A}(t)}: \mathcal{A}(t) \rightarrow \mathbb{R}$ nos pontos fora de $A(t) \cup A^{*}(t)$.

Dado $x \in \mathcal{A}(t) \backslash\left(A(t) \cup A^{*}(t)\right)$, uma vez que $x \notin A^{*}(t)$ segue-se que $\lim _{r \rightarrow \infty} d(T(r+$ $t, t) x, A(r+t))=0$ e como $x \notin A(t)$ tem-se $l(t, x)>0$. É fácil ver, do fato de que $|l(t, x)-l(t, y)| \leq \frac{2}{d_{0}} d(x, y)$, para todos $x, y \in X$ e todo real $t$, que podemos escolher 
$\delta>0$ de maneira que $l_{s}\left(\mathcal{O}_{\delta}(A(s))\right) \subset\left[0, \frac{l(t, x)}{2}\right)$ para todo real $s$ e, graças ao Lema 7.3.3, podemos encontrar $\delta^{\prime} \in(0, \delta)$ tal que

$$
T(r+t, t)\left(\mathcal{A}(t) \cap \mathcal{O}_{\delta^{\prime}}(A(t))\right) \subset \mathcal{A}(r+t) \cap \mathcal{O}_{\delta}(A(r+t)) \text { para todo } r \geq 0 .
$$

Do fato de que $\lim _{r \rightarrow \infty} d(T(r+t, t) x, A(r+t))=0$, conluímos a existência de $\sigma>0$ com a propriedade de que $T(r+t, t) x \in \mathcal{O}_{\delta^{\prime}}(A(r+t))$, toda vez que $r \geq \sigma$. Por um lado, pela continuidade de $T(\sigma+t, t): X \rightarrow X$, existe uma vizinhança $U_{1}$ de $x$ em $X$ tal que $T(\sigma+t, t) U_{1} \subset \mathcal{O}_{\delta^{\prime}}(A(\sigma+t))$. Então, para todo $z \in \mathcal{A}(t) \cap U_{1}$ temos $T(\sigma+t, t) z \in \mathcal{O}_{\delta^{\prime}}(A(\sigma+t))$ donde segue-se, levando em conta (7.4.13), que $T(r+t, t) z \in$ $\mathcal{A}(r+t) \cap \mathcal{O}_{\delta}(A(r+t))$ sempre que $r \geq \sigma$ e $z \in U_{1}$ e por isso $\sup _{r \geq \sigma} l(r+t, T(r+t, t) z) \leq$ $\frac{l(t, x)}{2}$ sempre que $z \in U_{1}$.

Finalmente, pela continuidade de $l_{t}$, seja $U_{2}$ uma vizinhança de $x$ em $X$ de maneira que $l(t, z)>\frac{l(t, x)}{2}$ para todo $z \in U_{2}$ e definamos $U:=U_{1} \cap U_{2} \cap \mathcal{A}(t)$, então para todo $z \in U$ temos $k(t, z)=\sup _{0 \leq r \leq \sigma} l(r+t, T(r+t, t) z)$, donde obtemos a continuidade de $\left.k_{t}\right|_{\mathcal{A}(t)}$ em $\mathcal{A}(t)$ da mesma maneira que concluímos a continuidade da função " $k$ " que aparece na Proposição 4.2.2 e assim esta resulta demonstrada.

A prova do próximo resultado é similar à da última proposição e segue as mesmas idéias da demonstração da Proposição 4.2.2. Ele nos traz um caso especial onde se verifica a continuidade, em ambas as duas variáveis, da função de Lyapunov de um par atrator-repulsor (não autônomo).

Observemos que o último resultado nos diz apenas que a função de Lyapunov do par atrator-repulsor $\left(A, A^{*}\right)$ é contínua, somente, na seção $\mathcal{A}(t)$ do atrator pullback no instante fixado $t$.

Proposição 7.4.4. Seja $\left(A, A^{*}\right)$ um par atrator-repulsor para um processo de evolução $\{T(t, s): t \geq s\}$ num espaço métrico $X$ possuindo atrator pullback $\{\mathcal{A}(t): t \in \mathbb{R}\}$. Suponhamos que para algum $\varepsilon_{0}>0$

$$
\mathcal{O}_{\varepsilon_{0}}(A(t)) \cap \mathcal{O}_{\varepsilon_{0}}\left(A^{*}(t)\right)=\varnothing, \text { para cada } t \in \mathbb{R} .
$$

Suponhamos também que se verifiquem as seguintes duas condições:

(a) A conclusão do Lema 7.3.3 é válida considerando-se vizinhanças de A em X. Em símbolos, que para cada $\delta>0$ existe $\delta^{\prime} \in(0, \delta)$ tal que

$$
T(r+t, t)\left(\mathcal{O}_{\delta^{\prime}}(A(t))\right) \subset \mathcal{O}_{\delta}(A(r+t)), \text { para todo } r \geq 0 \text { e todo } t \in \mathbb{R} .
$$

(b) Para cada $t \in \mathbb{R}$ e $x \in X \backslash\left(A(t) \cup A^{*}(t)\right)$ tem-se que

$$
\text { ou } \lim _{r \rightarrow \infty} d(T(r+t, t) x, A(r+t))=0 \text { ou } \lim _{r \rightarrow \infty} d\left(T(r+t, t) x, A^{*}(r+t)\right)=0 \text {. }
$$


Nestas condições, a função $k: \mathbb{R} \times X \rightarrow \mathbb{R}$, definida na Proposição \%.4.3, é contínua (em ambas as variáveis $(t, x)$ e em todos os pontos de $\mathbb{R} \times X$ ).

Demonstração: De fato, dado $\left(t_{0}, x_{0}\right) \in \mathbb{R} \times X$, como na Proposição 7.4.3, dividimos esta prova em três situações:

Caso 1) $x_{0} \in A^{*}\left(t_{0}\right)$.

Como $l(t, x) \leq k(t, x) \leq 1$, para todo $t \in \mathbb{R}$ e $x \in X$ podemos escrever

$$
\left|k(t, x)-k\left(t_{0}, x_{0}\right)\right|=1-k(t, x) \leq 1-l(t, x)
$$

Isto e a continuidade em ambas as variáveis da função $l: \mathbb{R} \times X \rightarrow \mathbb{R}$ (estabelecida na proposição anterior) em $\left(t_{0}, x_{0}\right)$ implicam a continuidade de $k: \mathbb{R} \times X \rightarrow \mathbb{R}$ em $\left(t_{0}, x_{0}\right)$.

Caso 2) $x_{0} \in A\left(t_{0}\right)$. De

$$
|l(t, x)-l(t, z)| \leq \frac{2}{\varepsilon_{0}} d(x, z), \text { para todos } x, z \in X \text { e } t \in \mathbb{R},
$$

dado $\varepsilon>0$, existe $\delta>0$ de maneira que $l\left(s, \mathcal{O}_{\delta}(A(s))\right) \subset[0, \varepsilon)$ para todo $s \in \mathbb{R}$.

Por outro lado, pela hipótese $(a)$, existe $\delta^{\prime} \in(0, \delta)$ tal que $T(r+t, t)\left(\mathcal{O}_{\delta^{\prime}}(A(t))\right) \subset$ $\mathcal{O}_{\delta}(A(r+t))$, para todo $r \geq 0$ e $t \in \mathbb{R}$, donde conclui-se que $k\left(t, \mathcal{O}_{\delta^{\prime}}(A(t))\right) \subset[0, \varepsilon]$ para cada real $t$.

Agora, pelo Lema 7.4.2, seja $\delta^{\prime \prime}>0$ de modo que $A\left(t_{0}\right) \subset \mathcal{O}_{\frac{\delta^{\prime}}{2}}(A(t))$, e por isto $\mathcal{O}_{\frac{\delta^{\prime}}{2}}\left(A\left(t_{0}\right)\right) \subset \mathcal{O}_{\delta^{\prime}}(A(t))$, toda vez que $\left|t-t_{0}\right|<\delta^{\prime \prime}$. Portanto, para $\left|t-t_{0}\right|<\delta^{\prime \prime} \mathrm{e}$ $d\left(x, x_{0}\right)<\frac{\delta^{\prime}}{2}$ tem-se $k(t, x) \leq \varepsilon$. O que dá a continuidade de $k: \mathbb{R} \times X \rightarrow \mathbb{R}$ no ponto $\left(t_{0}, x_{0}\right)$ quando $x_{0} \in A\left(t_{0}\right)$.

Caso 3) $x_{0} \in X \backslash\left(A\left(t_{0}\right) \cup A^{*}\left(t_{0}\right)\right)$.

Neste caso, graças à hipótese $(b)$, tem-se que

$$
\text { ou } \lim _{r \rightarrow \infty} d\left(T\left(r+t_{0}, t_{0}\right) x_{0}, A\left(r+t_{0}\right)\right)=0 \text { ou } \lim _{r \rightarrow \infty} d\left(T\left(r+t_{0}, t_{0}\right) x_{0}, A^{*}\left(r+t_{0}\right)\right)=0 \text {. }
$$

Primeiramente, suponhamos que seja $\lim _{r \rightarrow \infty} d\left(T\left(r+t_{0}, t_{0}\right) x_{0}, A\left(r+t_{0}\right)\right)=0$, como $x_{0} \notin A^{*}\left(t_{0}\right)$ segue-se que $l\left(t_{0}, x_{0}\right)>0$. Escolhamos então, por (7.4.14), $\delta>0$ de modo que $l\left(s, \mathcal{O}_{\delta}(A(s))\right) \subset\left[0, \frac{l\left(t_{0}, x_{0}\right)}{2}\right)$ para todo $s \in \mathbb{R}$ e, pela hipótese $(a)$, escolhamos, além disso, $\delta^{\prime} \in(0, \delta)$ tal que $T(r+t, t)\left(\mathcal{O}_{\delta^{\prime}}(A(t))\right) \subset \mathcal{O}_{\delta}(A(r+t))$, sempre que $r \geq 0$.

Levando em conta $\lim _{r \rightarrow \infty} d\left(T\left(r+t_{0}, t_{0}\right) x_{0}, A\left(r+t_{0}\right)\right)=0$, existe $\sigma>0$ com a propriedade de que $T\left(\sigma+t_{0}, t_{0}\right) x_{0} \in \mathcal{O}_{\frac{\delta^{\prime}}{2}}\left(A\left(\sigma+t_{0}\right)\right)$. Pela continuidade do processo $\{T(t, s): t \geq s\}$ e pelo Lema 7.4.2, existem uma vizinhança $U_{1}$ de $x_{0}$ em $X$ e $\delta^{\prime \prime}>0$ tais que $T(\sigma+t, t) U_{1} \subset \mathcal{O}_{\frac{\delta^{\prime}}{2}}\left(A\left(\sigma+t_{0}\right)\right) \subset \mathcal{O}_{\delta^{\prime}}(A(\sigma+t))$ toda vez que $\left|t-t_{0}\right|<\delta^{\prime \prime}$. Consequentemente, para todo $x \in U_{1}$ e todo $t \in\left(t_{0}-\delta^{\prime \prime}, t_{0}+\delta^{\prime \prime}\right)$, temos $T(\sigma+t, t) x \in \mathcal{O}_{\delta^{\prime}}(A(\sigma+t))$, logo $T(r+t, t) x \in \mathcal{O}_{\delta}(A(r+t))$ sempre que $r \geq \sigma$, $x \in U_{1}$ e $t \in\left(t_{0}-\delta^{\prime \prime}, t_{0}+\delta^{\prime \prime}\right)$. 
Agora, pela continuidade de $l: \mathbb{R} \times X \rightarrow \mathbb{R}$, sejam $U_{2}$ uma vizinhança de $x_{0}$ em $X$ e $0<\eta<\delta^{\prime \prime}$ tais que $l(t, x)>\frac{l\left(t_{0}, x_{0}\right)}{2}$ para todo $x \in U_{2}$ e todo $\left|t-t_{0}\right|<\eta$ e ponhamos $U:=U_{1} \cap U_{2}$. Daí segue-se que, para todo $x \in U$ e todo $t$ com $\left|t-t_{0}\right|<\eta$, tem-se $k(t, x)=\sup _{0 \leq r \leq \sigma} l(r+t, T(r+t, t) x)$.

Então, dado $\varepsilon>0$, de (7.4.14), existe $\mu_{1}>0$ tal que

$$
l(s, z)-\frac{\varepsilon}{2}<l(s, w)<l(s, z)+\frac{\varepsilon}{2},
$$

toda vez que $d(z, w)<\mu_{1}$ e $s \in \mathbb{R}$ é qualquer.

Por outro lado, mais uma vez pela continuidade do processo $T(\cdot, \cdot)$, é possível encontrar $\mu_{2} \in(0, \eta)$ tal que $d\left(T(r+t, t) x, T\left(r+t_{0}, t_{0}\right) x_{0}\right)<\mu_{1}$ sempre que $\left|t-t_{0}\right|<\mu_{2}$ e $d\left(x, x_{0}\right)<\mu_{2}$, com $r \in[0, \sigma]$.

Assim, se escolhemos $\mu_{2}>0$ suficientemente pequeno de forma que $d\left(x, x_{0}\right)<\mu_{2}$ implique $x \in U$, obtemos, de (7.4.15), que para $d\left(x, x_{0}\right)<\mu_{2}$ e $\left|t-t_{0}\right|<\mu_{2}$

$$
l\left(r+t_{0}, T\left(r+t_{0}, t_{0}\right) x_{0}\right)-\frac{\varepsilon}{2}<l(r+t, T(r+t, t) x)<l\left(r+t_{0}, T\left(r+t_{0}, t_{0}\right) x_{0}\right)+\frac{\varepsilon}{2}
$$

e por isto,

$\sup _{0 \leq r \leq \sigma} l\left(r+t_{0}, T\left(r+t_{0}, t_{0}\right) x_{0}\right)-\frac{\varepsilon}{2} \leq \sup _{0 \leq r \leq \sigma} l(r+t, T(r+t, t) x) \leq \sup _{0 \leq r \leq \sigma} l\left(r+t_{0}, T\left(r+t_{0}, t_{0}\right) x_{0}\right)+\frac{\varepsilon}{2}$, ou seja,

$$
k\left(t_{0}, x_{0}\right)-\frac{\varepsilon}{2} \leq k(t, x) \leq k\left(t_{0}, x_{0}\right)+\frac{\varepsilon}{2} .
$$

O que dá a continuidade de $k$ em pontos $\left(t_{0}, x_{0}\right)$ com $x_{0} \in X \backslash\left(A\left(t_{0}\right) \cup A^{*}\left(t_{0}\right)\right)$ e $\lim _{r \rightarrow \infty} d\left(T\left(r+t_{0}, t_{0}\right) x_{0}, A\left(r+t_{0}\right)\right)=0$.

Finalmente, se $\lim _{r \rightarrow \infty} d\left(T\left(r+t_{0}, t_{0}\right) x_{0}, A^{*}\left(r+t_{0}\right)\right)=0$ então $k\left(t_{0}, x_{0}\right)=1$, e dado $\varepsilon>0$ podemos escolher $\delta>0$ tal que $l\left(s, \mathcal{O}_{\delta}\left(A^{*}(s)\right)\right) \subset(1-\varepsilon, 1]$ para todo real $s$. Fixemos $\sigma>0 \operatorname{com} T\left(\sigma+t_{0}, t_{0}\right) x_{0} \in \mathcal{O}_{\frac{\delta}{2}}\left(A^{*}\left(\sigma+t_{0}\right)\right)$.

Pela continuidade do processo $\{T(t, s): t \geq s\}$ e pelo Lema 7.4.2, existem uma vizinhança $U$ de $x_{0}$ em $X$ e $\delta^{\prime}>0$ tais que $T(\sigma+t, t) U \subset \mathcal{O}_{\frac{\delta}{2}}\left(A^{*}\left(\sigma+t_{0}\right)\right) \subset \mathcal{O}_{\delta}\left(A^{*}(\sigma+t)\right)$ sempre que $\left|t-t_{0}\right|<\delta^{\prime}$, portanto, $1-\varepsilon<l(\sigma+t, T(\sigma+t, t) x) \leq k(t, x) \leq 1$ toda vez que $x \in U$ e $\left|t-t_{0}\right|<\delta^{\prime}$ completando a prova da proposição.

O seguinte lema traz o análogo não autônomo do Lema 4.2.1 que, como antes, nos ajudará a estender as propriedades da função $k$, dada no último resultado, para todo o espaço de fase $X$.

Lema 7.4.5. Seja $\{T(t, s): t \geq s\}$ um processo de evolução num espaço métrico $X$, possuindo atrator pullback $\{\mathcal{A}(t): t \in \mathbb{R}\}$ tal que para cada $x \in X$ e cada $t \in \mathbb{R}$

$$
\lim _{r \rightarrow \infty} d(T(r+t, t) x, \mathcal{A}(r+t))=0,
$$


e que para todo $\delta>0$ existe $\delta^{\prime} \in(0, \delta)$ de modo que

$$
T(r+t, t)\left(\mathcal{O}_{\delta^{\prime}}(\mathcal{A}(t))\right) \subset \mathcal{O}_{\delta}(\mathcal{A}(r+t)) \text {, para todo } r \geq 0 \text { e } t \in \mathbb{R} .
$$

Então, a função $h: \mathbb{R} \times X \rightarrow \mathbb{R}$ definida por

$$
h(t, x):=\sup _{r \geq 0} d(T(r+t, t) x, \mathcal{A}(r+t)),(t, x) \in \mathbb{R} \times X,
$$

é contínua (nas duas variáveis) e para cada $(t, x) \in \mathbb{R} \times X$, a função real $[0, \infty) \ni r \mapsto$ $h(r+t, T(r+t, t) x) \in \mathbb{R}$ é não crescente com $h(t, x)=0$ se, e somente se, $x \in \mathcal{A}(t)$.

Demonstração: Com efeito, a prova do fato de que a função $[0, \infty) \ni r \mapsto h(r+$ $t, T(r+t, t) x) \in \mathbb{R}$ é não crescente para cada $x \in X$ e $t \in \mathbb{R}$, é inteiramente análoga à prova da mesma propriedade da função $k: \mathbb{R} \times X \rightarrow \mathbb{R}$ dada na Proposição 7.4.3 e a prova da continuidade pode-se fazer, utilizando as duas hipóteses (7.4.16) e (7.4.17), da mesma maneira que fizemos os Casos 2) e 3) na prova da continuidade de $k$ na Proposição 7.4.4.

Nas condições dos dois últimos resultados e usando as idéias desenvolvidas no caso autônomo podemos melhorar a conclusão da Proposição 7.4.3 demonstrando o seguinte teorema.

Teorema 7.4.6. Seja $\{T(t, s): t \geq s\}$ um processo de evolução em um espaço métrico $X$, possuindo atrator pullback $\{\mathcal{A}(t): t \in \mathbb{R}\}$ tal que para cada $x \in X$ e cada $t \in \mathbb{R}$

$$
\lim _{r \rightarrow \infty} d(T(r+t, t) x, \mathcal{A}(r+t))=0
$$

e para todo $\delta>0$ existe $\delta^{\prime} \in(0, \delta)$ tal que

$$
T(r+t, t)\left(\mathcal{O}_{\delta^{\prime}}(\mathcal{A}(t))\right) \subset \mathcal{O}_{\delta}(\mathcal{A}(r+t)), \text { para todo } r \geq 0 \text { e } t \in \mathbb{R} .
$$

Seja também $\left(A, A^{*}\right)$ um par atrator-repulsor para $\{T(t, s): t \geq s\}$ com

$$
\mathcal{O}_{\varepsilon}(A(t)) \cap \mathcal{O}_{\varepsilon}\left(A^{*}(t)\right)=\varnothing, \text { para todo } t \in \mathbb{R},
$$

e algum $\varepsilon>0$ e, além disso, satisfazendo as seguintes condições:

(a) A conclusão do Lema 7.3.3 é válida considerando-se vizinhanças de $A$ em $X$, isto é, para cada $\delta>0$ existe $\delta^{\prime} \in(0, \delta)$ tal que

$$
T(r+t, t)\left(\mathcal{O}_{\delta^{\prime}}(A(t))\right) \subset \mathcal{O}_{\delta}(A(r+t)) \text {, para todo } r \geq 0 \text { e } t \in \mathbb{R} .
$$

(b) Para cada $t \in \mathbb{R}$ e $x \in X \backslash\left(A(t) \cup A^{*}(t)\right)$ tem-se que

$$
\text { ou } \lim _{r \rightarrow \infty} d(T(r+t, t) x, A(r+t))=0 \text { ou } \lim _{r \rightarrow \infty} d\left(T(r+t, t) x, A^{*}(r+t)\right)=0 .
$$

Então, existe uma função $f: \mathbb{R} \times X \rightarrow \mathbb{R}$ possuindo as seguintes quatro propriedades: 
(i) Para cada par $(t, x) \in \mathbb{R} \times X$, a função $[0, \infty) \ni r \mapsto f(r+t, T(r+t, t) x) \in \mathbb{R}$ é não crescente.

(ii) Se $t \in \mathbb{R}$ e $f_{t}: X \rightarrow \mathbb{R}$ é definida por $f_{t}(x):=f(t, x)$, para $x \in X$, então $f_{t}^{-1}(0)=A(t)$ e $f_{t}^{-1}(1) \cap \mathcal{A}(t)=A^{*}(t)$.

(iii) Dados $t \in \mathbb{R}$ e $x \in X$, se $f(r+t, T(r+t, t) x)=f(t, x)$ para todo $r \geq 0$, então $x \in A(t) \cup A^{*}(t)$.

(iv) $f: \mathbb{R} \times X \rightarrow \mathbb{R}$ é contínua (em ambas as variáveis).

Demonstração: Com efeito, sejam $k: \mathbb{R} \times X \rightarrow \mathbb{R}$ a função dada na Proposição 7.4.4 e $h: \mathbb{R} \times X \rightarrow \mathbb{R}$ a dada no Lema 7.4.5. Definimos $f: \mathbb{R} \times X \rightarrow \mathbb{R}$ por

$$
f(t, x):=k(t, x)+h(t, x),(t, x) \in \mathbb{R} \times X .
$$

Segue-se, imediatamente das propriedades de $h$ e $k$, que $f$ é contínua (nas duas variáveis) e que, para cada $(t, x) \in \mathbb{R} \times X$, a função $[0, \infty) \ni r \mapsto f(r+t, T(r+t, t) x) \in \mathbb{R}$ é não crescente.

Para provar (ii), primeiramente seja $x \in A(t) \subset \mathcal{A}(t)$ para um certo número real fixado $t$, então $h(t, x)=0$ e, pela definição de $k: \mathbb{R} \times X \rightarrow \mathbb{R}$, temos $k(t, x)=0$. Portanto, $f(t, x)=0$ e por isto $A(t) \subset f_{t}^{-1}(0)$. Reciprocamente, se $x \in X$ é tal que $f_{t}(x)=0$, então $h(t, x)=0$ e $k(t, x)=0$, donde $x \in \mathcal{A}(t)$ e por (ii) na Proposição 7.4.3 $x \in A(t)$. O que prova que $f_{t}^{-1}(0) \subset A(t)$. Por outro lado, se $x \in A^{*}(t) \subset \mathcal{A}(t)$ tem-se $h(t, x)=0$, consequentemente $f(t, x)=k(t, x)+h(t, x)=k(t, x)=1$, pelas propriedades de $k$. Daí conclui-se que $A^{*}(t) \subset f^{-1}(1) \cap \mathcal{A}(t)$. Agora, seja $x \in \mathcal{A}(t)$ com $f(t, x)=1$, donde $h(t, x)=0$ e $k(t, x)=1$ portanto $x \in A^{*}(t)$ e a prova de $(i i)$ está completa.

A demonstração de (iii) pode-se realizar assim: Seja $(t, x) \in \mathbb{R} \times X$ com

$$
f(r+t, T(r+t, t) x)=f(t, x) \text { para todo } r \geq 0 .
$$

Se $x \in \mathcal{A}(t)$ temos $h(r+t, T(r+t, t) x)=0$ para todo $r \geq 0$ e, então, $k(r+t, T(r+$ $t, t) x)=k(t, x)$ para todo $r \geq 0$ logo, graças a $($ iii $)$ na Proposição 7.4.3, a conclusão segue.

Agora, provemos que a única maneira de (7.4.18) ocorrer é quando $x \in \mathcal{A}(t)$. Com efeito, se $x \in X \backslash \mathcal{A}(t)$ então $\lim d\left(T(r+t, t) x, A^{*}(r+t)\right)=0$, porque, se este não é o

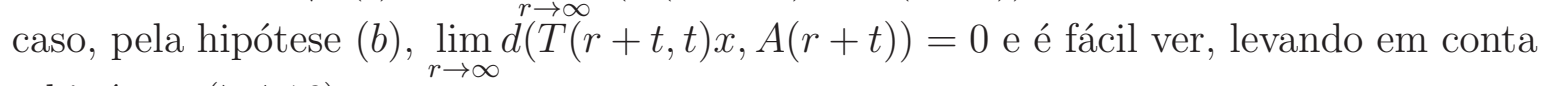
a hipótese (7.4.16), que

$$
\begin{aligned}
f(t, x) & =\lim _{r \rightarrow \infty} f(r+t, T(r+t, t) x) \\
& =\lim _{r \rightarrow \infty} k(r+t, T(r+t, t) x)+\lim _{r \rightarrow \infty} h(r+t, T(r+t, t) x)=0,
\end{aligned}
$$


assim, como $k$ e $h$ são ambas funções não negativas, devemos ter $k(t, x)=0$ e $x \in A(t)$, o que contradiz o fato de que $x \in X \backslash \mathcal{A}(t)$. Daí conclui-se que $\lim _{r \rightarrow \infty} d\left(T(r+t, t) z, A^{*}(r+\right.$ $t))=0$ é certo. Agora, utilizando-se o mesmo raciocínio usado em (7.4.19), obtém-se

$$
\begin{aligned}
f(t, x) & =\lim _{r \rightarrow \infty} f(r+t, T(r+t, t) x) \\
& =\lim _{r \rightarrow \infty} k(r+t, T(r+t, t) x)+\lim _{r \rightarrow \infty} h(r+t, T(r+t, t) x)=1+0=1,
\end{aligned}
$$

mas isso não pode ser verdade, porque como $k(t, x) \geq k(r+t, T(r+t, t) x)$ sempre que $r \geq 0$ deveríamos ter $1 \geq k(t, x) \geq \lim _{r \rightarrow \infty} k(r+t, T(r+t, t) x)=1$, portanto $h(t, x)=0 \mathrm{e}$ $k(t, x)=1$, e assim $x \in A^{*}(t)$, contradizendo $x \in X \backslash \mathcal{A}(t)$ e a prova está completa.

Observemos que, assim como no caso autônomo, o papel da função $h: \mathbb{R} \times X \rightarrow \mathbb{R}$, na prova do teorema acima, é o de reconhecer os pontos de $X$ que fazem com que $f$ estacione, ou seja, pontos $x$ tais que $f(r+t, T(r+t, t) x)=f(t, x)$ para todo $r \geq 0$ e um certo real $t$, como sendo apenas os pontos da seção $\mathcal{A}(t)$ do atrator pullback. Propriedade que a função $k$, sozinha, poderia não possuir.

Finalmente, reunindo-se todos os resultados que desenvolvemos até agora, podemos enunciar o próximo teorema cuja demonstração é análoga à do Teorema 4.4.1 do Capítulo 4.

Teorema 7.4.7. Seja $\{T(t, s): t \geq s\}$ um processo de evolução de tipo gradiente com respeito ao conjunto finito de famílias invariantes isoladas $\boldsymbol{\Xi}=\left\{\Xi_{1}, \cdots, \Xi_{n}\right\}$ limitadas possuindo atrator pullback $\mathcal{A}=\{\mathcal{A}(t): t \in \mathbb{R}\}$. Seja também, $\sigma: I_{n} \rightarrow I_{n}$ uma permutação tal que a n-upla ordenada $\mathbf{D}=\left(\Xi_{\sigma(1)}, \Xi_{\sigma(2)}, \cdots, \Xi_{\sigma(n)}\right)$ é, segundo a hipótese (S1) abaixo, uma decomposição de Morse para o atrator $\mathcal{A}$, segundo o Teorema 7.3.9.

Seja, para $j=1,2, \cdots, n$ e $t \in \mathbb{R}, A_{j}(t)=\bigcup_{i=1}^{j} W^{u}\left(\Xi_{\sigma(i)}\right)(t)$.

Suponhamos que as seguintes condições estejam satisfeitas:

(S1) Existe $\varepsilon_{0}>0$ tal que $\mathcal{O}_{\varepsilon_{0}}\left(A_{j}(t)\right) \cap \mathcal{O}_{\varepsilon_{0}}\left(A_{j}^{*}(t)\right)=\varnothing$, para todo $j=1,2, \cdots, n$ e $t \in \mathbb{R}$,

(S2) Dado $\delta>0$, existe $\delta^{\prime} \in(0, \delta)$ tal que

$$
T(r+t, t)\left(\mathcal{A}(t) \cap \mathcal{O}_{\delta^{\prime}}\left(A_{j}(t)\right)\right) \subset \mathcal{A}(r+t) \cap \mathcal{O}_{\delta}\left(A_{j}(r+t)\right)
$$

para todo $r \geq 0, j=1,2, \cdots, n$ e $t \in \mathbb{R}$.

Então, existe uma função de Lyapunov $V: \mathbb{R} \times X \rightarrow \mathbb{R}$ com as propriedades (i)-(iii) da Definição 7.4.1 de tal forma que $V\left(t, \Xi_{\sigma(i)}(t)\right)=\{i\}$, para todo $t \in \mathbb{R}$ e $i=1, \cdots, n$. 
Além disso, se (S2) é válido para vizinhanças dos $A_{j}{ }^{\prime}$ s em $X$, as hipóteses sobre o atrator pullback exigidas no Lema 7.4 .5 estão satisfeitas e dados $(t, x) \in \mathbb{R} \times X$ e $j=1,2, \cdots, n \lim _{r \rightarrow \infty} d\left(T(r+t, t) x, A_{j}(r+t) \cup A_{j}^{*}(r+t)\right)=0$, então a função de Lyapunov $V: \mathbb{R} \times X \rightarrow \mathbb{R}$ pode ser tomada contínua em ambas as variáveis.

\subsection{Perturbação não autônoma de um semigrupo gra- diente}

Como vimos na seção anterior, a fim de se desenvolver, comparando com o caso dos semigrupos, a teoria de Morse e, consequentemente, construir as funções de Lyapunov para os processos não autônomos, fez-se necessário impor certas restrições sobre o comportamento de alguns objetos relacionados ao sistema. Nestas condições, esta seção tem como objetivo exibir uma classe de exemplos onde se encontram satisfeitas estas restrições e, portanto, onde os resultados anteriores possam ser aplicados. Esta classe é formada pelas perturbações não autônomas pequenas de semigrupos gradientes.

Comecemos apresentando o Teorema 3.9 em [8], cuja prova será aqui omitida por ser análoga à prova do Teorema 3.2.4 dada no Capítulo 3.

Assinalemos que, ainda que tudo o que fazemos nesta seção esteja restrito ao caso onde as famílias invariantes isoladas consideradas sejam dadas por soluções globais e a família de invariantes isolados do semigrupo limite sendo um conjunto finito de pontos de equilíbrios, não existe diferença alguma (que não seja puramente relacionada a notação) entre as provas que apresentamos neste caso e a do caso onde se consideram famílias invariantes isoladas mais gerais. Escolhemos fazer este caso "mais simples" somente por questões de notação.

Teorema 7.5.1 (Carvalho e Langa). Seja $X$ um espaço métrico e, para cada $\eta \in[0,1]$, $\left\{T_{\eta}(t, s): t \geq s\right\}$ um processo de evolução em $X$ possuindo atrator pullback $\left\{\mathcal{A}_{\eta}(t)\right.$ : $t \in \mathbb{R}\}$.

Suponhamos que estejam satisfeitas as seguintes condições:

(a) $\overline{\bigcup_{\eta \in[0,1]} \bigcup_{t \in \mathbb{R}} \mathcal{A}_{\eta}(t)}$ é compacto.

(b) $\left\{T_{0}(t, s): t \geq s\right\}$ é um processo de evolução autônomo com $T_{0}(t, s)=S(t-s)$ para todo $t \geq s$ e um certo semigrupo $\{S(t): t \geq 0\}$ de tipo gradiente com respeito ao conjunto finito de pontos de equílibrio $\mathcal{E}_{0}:=\left\{z_{1,0}^{*}, \cdots, z_{n, 0}^{*}\right\}$. 
(c) Para cada $\eta \in(0,1],\left\{T_{\eta}(t, s): t \geq s\right\}$ possui $n$ soluções globais isoladas ${ }^{2} \xi_{i, \eta}^{*}$ : $\mathbb{R} \rightarrow X, i=1,2, \cdots, n, \eta \in(0,1]$, tais que $\lim _{\eta \rightarrow 0+} \sup _{1 \leq i \leq n} \sup _{t \in \mathbb{R}} d\left(\xi_{i, \eta}^{*}(t), z_{i, 0}^{*}\right)=0$.

(d) Para cada compacto $K \subset \mathbb{R}^{+} \times X$

$$
\lim _{\eta \rightarrow 0^{+}} \sup _{s \in \mathbb{R}} \sup _{(t, x) \in K} d\left(T_{\eta}(t+s, s) x, T_{0}(t+s, s) x\right)=0 .
$$

(e) Existe uma constante $\mu>0$ tal que, se $\xi_{\eta}: \mathbb{R} \rightarrow X$ é uma solução global limitada de $\left\{T_{\eta}(t, s): t \geq s\right\}$ tal que para algum $t_{0} \in \mathbb{R}$ e algum $i=1, \cdots, n$ $\sup _{t \leq t_{0}} d\left(\xi_{\eta}(t), \xi_{i, \eta}^{*}(t)\right)<\mu\left(\operatorname{resp} . \sup _{t \geq t_{0}} d\left(\xi_{\eta}(t), \xi_{i, \eta}^{*}(t)\right)<\mu\right)$, então $\lim _{t \rightarrow-\infty} d\left(\xi_{\eta}(t), \xi_{i, \eta}^{*}(t)\right)=$ 0 (resp. $\left.\lim _{t \rightarrow \infty} d\left(\xi_{\eta}(t), \xi_{i, \eta}^{*}(t)\right)=0\right)$.

Então, existe $\eta_{0} \in(0,1]$ tal que para cada $\eta \in\left(0, \eta_{0}\right],\left\{T_{\eta}(t, s): t \geq s\right\}$ é um processo de evolução de tipo gradiente com respeito ao conjunto finito de soluções globais isoladas $\mathcal{E}_{\eta}=\left\{\xi_{1, \eta}^{*}, \cdots, \xi_{n, \eta}^{*}\right\}$.

A seguir trabalhamos com famílias de processos que satisfazem uma certa condição de compacidade coletiva no infinito, mais precisamente, famílias que verificam a condição da seguinte definição:

Definição 7.5.2. Uma família $\left\{T_{\eta}(t, s): t \geq s\right\}_{\eta \in[0,1]}$ de processos de evolução em um espaço métrico $X$ diz-se coletivamente assintoticamente compacta, quando a sequência $\left(T_{\eta_{k}}\left(t_{k}+\tau_{k}, \tau_{k}\right) x_{k}\right)_{k \in \mathbb{N}}$ possui subsequência convergente toda vez que $\left(\eta_{k}\right)_{k \in \mathbb{N}}$ é uma sequência em $[0,1]$, com $\eta_{k} \underset{k \rightarrow \infty}{\rightarrow} 0,\left(x_{k}\right)_{k \in \mathbb{N}}$ uma sequência limitada de pontos de $X e\left(t_{k}\right)_{k \in \mathbb{N}} e\left(\tau_{k}\right)_{k \in \mathbb{N}}$ sequências em $\mathbb{R}$ com $t_{k} \underset{k \rightarrow \infty}{\rightarrow} \infty$.

O seguinte lema técnico é crucial para estabelecer nossos próximos resultados, (confira o Lema 3.1 em [11] para uma prova, a qual é semelhante à prova do Lema 3.2.3 do Capítulo 3).

Lema 7.5.3. Seja $X$ um espaço métrico e $\left\{T_{\eta}(t, s): t \geq s\right\}_{\eta \in[0,1]}$ uma família de processos de evolução em $X$ possuindo atrator pullback $\left\{\mathcal{A}_{\eta}(t): t \in \mathbb{R}\right\}_{\eta \in[0,1]}$, satisfazendo as condições $(a),(b)$ e $(d)$ do Teorema 7.5.1.

Sejam também $\left(\eta_{k}\right)_{k \in \mathbb{N}}$ uma sequência em $[0,1]$ com $\eta_{k} \underset{k \rightarrow \infty}{\rightarrow} 0 e\left(t_{k}\right)_{k \in \mathbb{N}}$ uma sequência de números reais, então:

\footnotetext{
${ }^{2}$ Isto é, cada $\xi_{i, \eta}^{*}: \mathbb{R} \rightarrow X$ é uma solução global para o processo $T_{\eta}(\cdot, \cdot)$ e a família invariante dada por $\Xi_{i, \eta}:=\left\{\Xi_{i, \eta}(t): t \in \mathbb{R}\right\}, \Xi_{i, \eta}(t):=\left\{\xi_{i, \eta}^{*}(t)\right\}, t \in \mathbb{R}$ é isolada.
} 
(i) $S e\left(\xi_{k}\right)_{k \in \mathbb{N}}$ é uma sequência em $C(\mathbb{R} ; X)$ tal que, para cada $k \in \mathbb{N}, \xi_{k}: \mathbb{R} \rightarrow X$ é uma solução global limitada para $\left\{T_{\eta_{k}}(t, s): t \geq s\right\}$, então existe uma subsequência de $\left(\xi_{k}\right)_{k \in \mathbb{N}}$, que indicamos da mesma forma, e uma solução global $\xi: \mathbb{R} \rightarrow \mathcal{A}_{0}$ para $\{S(t): t \geq 0\}$, tal que

$$
\lim _{k \rightarrow \infty} \sup _{|t| \leq R} d\left(\xi_{k}\left(t+t_{k}\right), \xi(t)\right)=0, \text { para todo } R>0 .
$$

(ii) Se a família $\left\{T_{\eta}(t, s): t \geq s\right\}_{\eta \in[0,1]}$ é coletivamente assintoticamente compacta, $t_{k} \underset{k \rightarrow \infty}{\rightarrow} \infty,\left(\tau_{k}\right)_{k \in \mathbb{N}}$ uma sequência de números reais, $\left(x_{k}\right)_{k \in \mathbb{N}}$ uma sequência limitada de pontos de $X$ e $\zeta_{k}:\left[-t_{k}, \infty\right) \rightarrow X$ é dada por

$$
\zeta_{k}(t):=T_{\eta_{k}}\left(t+t_{k}+\tau_{k}, \tau_{k}\right) x_{k}, t \in\left[-t_{k}, \infty\right)
$$

então existe uma subsequência de $\left(\zeta_{k}\right)_{k \in \mathbb{N}}$, que também indicamos da mesma maneira, e uma solução global $\zeta: \mathbb{R} \rightarrow \mathcal{A}_{0}$ para $\{S(t): t \geq 0\}$, tal que

$$
\lim _{k \rightarrow \infty} \sup _{|t| \leq R} d\left(\zeta_{k}(t), \zeta(t)\right)=0, \text { para todo } R>0 \text {. }
$$

Com relação ao último lema observemos apenas que suas conclusões podem não se verificar no caso onde o processo limite $T_{0}(\cdot, \cdot)$ não provém de um semigrupo.

Seja $X$ um espaço métrico e, para cada $\eta \in[0,1],\left\{T_{\eta}(t, s): t \geq s\right\}$ um processo de evolução em $X$ com atrator pullback $\left\{\mathcal{A}_{\eta}(t): t \in \mathbb{R}\right\}$ satisfazendo as condições de $(a)$ a (e) do Teorema 7.5.1. Seja $\sigma: I_{n} \rightarrow I_{n}$ uma permutação tal que, levando em conta o Teorema 4.3.3 do Capítulo 4, a $n$-upla ordenada

$$
\mathbf{D}_{0}:=\left(z_{\sigma(1), 0}^{*}, z_{\sigma(2), 0}^{*}, \cdots, z_{\sigma(n), 0}^{*}\right)
$$

é uma decomposição de Morse para o atrator global $\mathcal{A}_{0}$ do semigrupo limite $\{S(t)$ : $t \geq 0\}$.

Nestas condições, para cada $\eta \in[0,1]$, consideremos a $n$-upla ordenada

$$
\mathbf{D}_{\eta}:=\left(\xi_{\sigma(1), \eta}^{*}, \xi_{\sigma(2), \eta}^{*}, \cdots, \xi_{\sigma(n), \eta}^{*}\right)
$$

que obtém-se utilizando-se no conjunto $\mathcal{E}_{\eta}=\left\{\xi_{1, \eta}^{*}, \cdots, \xi_{n, \eta}^{*}\right\}$ a mesma reordenação determinada pela permutação $\sigma: I_{n} \rightarrow I_{n}$. Observemos que, desta forma, evidentemente, tem-se

$$
\lim _{\eta \rightarrow 0+1 \leq i \leq n} \sup _{1 \in \mathbb{R}} d\left(\xi_{\sigma(i), \eta}^{*}(t), z_{\sigma(i), 0}^{*}\right)=0 .
$$

Agora seja, para cada $\eta \in[0,1], A_{0, \eta}(t):=\varnothing, A_{1, \eta}(t):=\left\{\xi_{\sigma(1), \eta}^{*}(t)\right\}$ e para cada real $t$ e $j=2, \cdots, n$

$$
A_{j, \eta}(t):=A_{j-1, \eta}(t) \cup W^{u}\left(\xi_{\sigma(j), \eta}^{*}\right)(t) .
$$


Também, para cada $\eta \in[0,1], j=2, \cdots, n$, e $t \in \mathbb{R}$, seja (não necessariamente, a priori, o repulsor complementar de nenhum atrator local)

$A_{j, \eta}^{*}(t):=\left\{x \in \mathcal{A}_{\eta}(t): d\left(T_{\eta}(r+t, t) x, A_{j, \eta}(r+t)\right)\right.$ não converge a zero quando $\left.r \rightarrow \infty\right\}$.

Provaremos, a seguir, que as famílias $A_{j, \eta}:=\left\{A_{j, \eta}(t): t \in \mathbb{R}\right\}$, como definidas acima, constituem, para $\eta>0$ suficientemente pequeno e $j$ qualquer, um atrator local para $T_{\eta}(\cdot, \cdot)$ e, consequentemente, os $A_{j, \eta}^{*}:=\left\{A_{j, \eta}^{*}(t): t \in \mathbb{R}\right\}$ serão seus repulsores complementares correspondentes. Além disso, provamos que todas as condições que impusemos quando construímos a decomposição de Morse para os processos de tipo gradiente e as funções de Lyapunov dos pares atrator-repulsor se satisfazem para estas famílias.

Comecemos provando a semicontinuidade superior destas famílias, mais precisamente, provemos o seguinte:

Lema 7.5.4. Consideremos $X$ um espaço métrico e, para cada $\eta \in[0,1],\left\{T_{\eta}(t, s)\right.$ : $t \geq s\}$ um processo de evolução em $X$ possuindo atrator pullback $\left\{\mathcal{A}_{\eta}(t): t \in \mathbb{R}\right\}$ satisfazendo as hipóteses de (a) a (e) do Teorema 7.5.1.

Seja $\sigma: I_{n} \rightarrow I_{n}$ uma permutação tal que a n-upla ordenada

$$
\mathbf{D}_{0}=\left(z_{\sigma(1), 0}^{*}, z_{\sigma(2), 0}^{*}, \cdots, z_{\sigma(n), 0}^{*}\right)
$$

é uma decomposição de Morse para o atrator global $\mathcal{A}_{0}$ do semigrupo limite $\{S(t): t \geq$ $0\}$ e consideremos, como acima, a n-upla ordenada

$$
\mathbf{D}_{\eta}=\left(\xi_{\sigma(1), \eta}^{*}, \xi_{\sigma(2), \eta}^{*}, \cdots, \xi_{\sigma(n), \eta}^{*}\right)
$$

Então, as famílias $A_{j, \eta}$ e $A_{j, \eta}^{*}$ definidas, respectivamente, em (7.5.20) e (7.5.21) se comportam semicontínuas superiormente quando $\eta \rightarrow 0$, ou seja, as convergências

$$
\lim _{\eta \rightarrow 0^{+}} \sup _{t \in \mathbb{R}} \operatorname{dist}\left(A_{j, \eta}(t), A_{j, 0}\right)=0
$$

$e$

$$
\lim _{\eta \rightarrow 0^{+}} \sup _{t \in \mathbb{R}} \operatorname{dist}\left(A_{j, \eta}^{*}(t), A_{j, 0}^{*}\right)=0,
$$

verificam-se para todo $j=1,2, \cdots, n$.

Demonstração: Com efeito, graças ao fato de que $A_{j, 0}$ e $A_{j, 0}^{*}$ são, para todo $j$, conjuntos compactos disjuntos, podemos escolher $\varepsilon>0$ tal que para cada $j=1,2, \cdots, n$

$$
\mathcal{O}_{\varepsilon}\left(A_{j, 0}\right) \cap \mathcal{O}_{\varepsilon}\left(A_{j, 0}^{*}\right)=\varnothing
$$


Primeiramente, provemos que para todo $j=1,2, \cdots, n$ temos $\lim _{\eta \rightarrow 0^{+}} \sup \operatorname{dist}\left(A_{j, \eta}(t), A_{j, 0}\right)=$ 0 .

Se este não é o caso, existem $\delta \in(0, \varepsilon), j=1,2, \cdots, n$, uma sequência $\left(\eta_{k}\right)_{k \in \mathbb{N}}$ em $(0,1]$ com $\eta_{k} \underset{k \rightarrow \infty}{\rightarrow} 0^{+}$, uma sequência $\left(\tau_{k}\right)_{k \in \mathbb{N}}$ em $\mathbb{R}$ e uma sequência $\left(x_{k}\right)_{k \in \mathbb{N}}$ de pontos de $X$ tais que, para cada $k \in \mathbb{N}, x_{k} \in A_{j, \eta_{k}}\left(\tau_{k}\right)$ e

$$
d\left(x_{k}, A_{j, 0}\right) \geq \delta
$$

Agora, pela definição dos conjuntos $A_{j, \eta}(t)$, podemos supor a existência de $i \leq j$, fixo, de modo que para cada natural $k$, existe uma solução global $\xi_{k}: \mathbb{R} \rightarrow X$ para $\left\{T_{\eta_{k}}(t, s): t \geq s\right\} \operatorname{com} \xi_{k}\left(\tau_{k}\right)=x_{k} \mathrm{e}$

$$
\lim _{t \rightarrow-\infty} d\left(\xi_{k}(t), \xi_{\sigma(i), \eta_{k}}^{*}(t)\right)=0 .
$$

Por outro lado, da hipótese $(c)$, segue a existência de um $\eta_{\delta}>0$ tal que para cada $\eta \leq \eta_{\delta}$ tem-se

$$
\sup _{t \in \mathbb{R}} d\left(\xi_{\sigma(i), \eta}^{*}(t), A_{j, 0}\right)<\frac{\delta}{2}
$$

Consequentemente, levando em conta (7.5.25) e (7.5.26), para cada $k$ existe um real $t_{k}$ tal que

$$
d\left(\xi_{k}(t), A_{j, 0}\right)<\delta \text { sempre que } t<t_{k},
$$

$\mathrm{e}$

$$
d\left(\xi_{k}\left(t_{k}\right), A_{j, 0}\right)=\delta
$$

Assim, definimos, para cada natural $k$, o caminho $\widetilde{\xi}_{k}: \mathbb{R} \rightarrow X$ pondo $\widetilde{\xi}_{k}(t):=$ $\xi_{k}\left(t+t_{k}\right), t \in \mathbb{R}$ e, pelo item $(i)$ do Lema 7.5.3, podemos supor a existência de uma solução global $\xi: \mathbb{R} \rightarrow X$ para o semigrupo $\{S(t): t \geq 0\}$ tal que, para cada $R>0$

$$
\lim _{k \rightarrow \infty} \sup _{|t| \leq R} d\left(\widetilde{\xi}_{k}(t), \xi(t)\right)=0 .
$$

De (7.5.27) resulta que $d\left(\xi(t), A_{j, 0}\right) \leq \delta$ sempre que $t \leq 0$, então por (7.5.24) e pelo fato de que $\{S(t): t \geq 0\}$ é de tipo gradiente com respeito a $\mathcal{E}_{0}=\left\{z_{1,0}^{*}, \cdots, z_{n, 0}^{*}\right\}$, necessariamente $\xi(0) \in A_{j, 0}$, mas (7.5.28) implica $d\left(\xi(0), A_{j, 0}\right)=\delta$ contradizendo $\xi(0) \in$ $A_{j, 0}$ e assim esta contradição prova 7.5.22.

Provemos agora que para cada $j=1,2, \cdots, n$ tem-se $\lim _{\eta \rightarrow 0^{+}} \sup \operatorname{dist}\left(A_{j, \eta}^{*}(t), A_{j, 0}^{*}\right)=0$.

Como antes, se esta conclusão é falsa, existem $\delta \in(0, \varepsilon), j=1,2, \cdots, n$, uma sequência $\left(\eta_{k}\right)_{k \in \mathbb{N}}$ em $(0,1] \operatorname{com} \eta_{k} \underset{k \rightarrow \infty}{\rightarrow} 0^{+}$, uma sequência $\left(\tau_{k}\right)_{k \in \mathbb{N}}$ em $\mathbb{R}$, e uma $\left(x_{k}\right)_{k \in \mathbb{N}}$ em $X$ tal que, para cada $k \in \mathbb{N}, x_{k} \in A_{j, \eta_{k}}^{*}\left(\tau_{k}\right)$ e

$$
d\left(x_{k}, A_{j, 0}^{*}\right) \geq \delta
$$


Pela definição dos conjuntos $A_{i, \eta}^{*}(t)$ 's (e também pelo Teorema 7.5.1), podemos supor a existência de um índice fixo $i \geq j+1$ tal que para cada $k \in \mathbb{N}$ existe uma solução global $\xi_{k}: \mathbb{R} \rightarrow X$ para o processo $\left\{T_{\eta_{k}}(t, s): t \geq s\right\} \operatorname{com} \xi_{k}\left(\tau_{k}\right)=x_{k} \mathrm{e}$

$$
\lim _{t \rightarrow \infty} d\left(\xi_{k}(t), \xi_{\sigma(i), \eta_{k}}^{*}(t)\right)=0 .
$$

Por outro lado, a hipótese $(c)$ acarreta a existência de um $\eta_{\delta}^{*}>0$ de maneira que para todo $\eta \leq \eta_{\delta}^{*}$ tem-se

$$
\sup _{t \in \mathbb{R}} d\left(\xi_{\sigma(i), \eta}^{*}(t), A_{j, 0}^{*}\right)<\frac{\delta}{2},
$$

donde, graças a (7.5.29) e a (7.5.30), para cada $k \in \mathbb{N}$, podemos escolher $t_{k} \in \mathbb{R}$ de modo que

$$
d\left(\xi_{k}(t), A_{j, 0}^{*}\right)<\delta \text { para todo } t>t_{k}
$$

e

$$
d\left(\xi_{k}\left(t_{k}\right), A_{j, 0}^{*}\right)=\delta
$$

Daí, podemos definir $\widetilde{\xi}_{k}: \mathbb{R} \rightarrow X$ pondo $\widetilde{\xi}_{k}(t):=\xi_{k}\left(t+t_{k}\right)$, para $t \in \mathbb{R}$, e assim, pelo item $(i)$ do Lema 7.5.3, podemos supor a existência de uma solução global $\xi: \mathbb{R} \rightarrow X$ para o semigrupo $\{S(t): t \geq 0\}$ tal que para cada $R>0$,

$$
\lim _{k \rightarrow \infty} \sup _{|t| \leq R} d\left(\widetilde{\xi}_{k}(t), \xi(t)\right)=0 .
$$

Agora, de (7.5.31) temos $d\left(\xi(t), A_{j, 0}^{*}\right) \leq \delta$ toda vez que $t \geq 0$ e, como uma consequência de (7.5.24), necessariamente $\xi(0) \in A_{j, 0}^{*}$. Mas (7.5.32) nos diz, após passar o limite com $k \rightarrow \infty$, que $d\left(\xi(0), A_{j, 0}^{*}\right)=\delta$, o que contradiz $\xi(0) \in A_{j, 0}^{*}$ e com isto o lema fica demonstrado.

A primeira condição que devemos verificar, a fim de provar que, para $\eta>0$ suficientemente pequeno, a $n$-upla ordenada $\mathbf{D}_{\eta}=\left(\xi_{\sigma(1), \eta}^{*}, \xi_{\sigma(2), \eta}^{*}, \cdots, \xi_{\sigma(n), \eta}^{*}\right)$ seja uma decomposição de Morse para o atrator pullback $\mathcal{A}_{\eta}=\left\{\mathcal{A}_{\eta}(t): t \in \mathbb{R}\right\}$, é a condição de separabilidade (7.3.8) no Teorema 7.3.9. E, de fato, a temos, como nos mostra a

Proposição 7.5.5. Seja $X$ um espaço métrico e, para cada $\eta \in[0,1],\left\{T_{\eta}(t, s): t \geq s\right\}$ um processo de evolução em $X$ possuindo atrator pullback $\left\{\mathcal{A}_{\eta}(t): t \in \mathbb{R}\right\}$ satisfazendo as hipóteses de (a) a (e) do Teorema 7.5.1.

Seja $\sigma: I_{n} \rightarrow I_{n}$ uma permutação tal que a n-upla ordenada

$$
\mathbf{D}_{0}=\left(z_{\sigma(1), 0}^{*}, z_{\sigma(2), 0}^{*}, \cdots, z_{\sigma(n), 0}^{*}\right)
$$

é uma decomposição de Morse para o atrator global $\mathcal{A}_{0}$ do semigrupo limite $\{S(t): t \geq$ $0\}$ e consideremos, como antes, a n-upla ordenada

$$
\mathbf{D}_{\eta}=\left(\xi_{\sigma(1), \eta}^{*}, \xi_{\sigma(2), \eta}^{*}, \cdots, \xi_{\sigma(n), \eta}^{*}\right) .
$$


Então, existem $\varepsilon>0$ e $\eta_{0}>0$ tais que

$$
\mathcal{O}_{\varepsilon}\left(A_{j, \eta}(t)\right) \cap \mathcal{O}_{\varepsilon}\left(\bigcup_{i=j+1}^{n}\left\{\xi_{\sigma(i), \eta}^{*}(t)\right\}\right)=\varnothing
$$

para cada $j=1,2, \cdots, n-1, t \in \mathbb{R}$ e $\eta \in\left[0, \eta_{0}\right]$, onde os conjuntos $A_{j, \eta}(t)$ 's estão definidos em (7.5.20) acima.

Demonstração: De fato, pela prova do Teorema 4.3 .3 (confira a condição (4.3.7)) podemos tomar $\varepsilon>0$ de modo que, para cada $j=1,2, \cdots, n-1$

$$
\mathcal{O}_{2 \varepsilon}\left(A_{j, 0}\right) \cap \mathcal{O}_{2 \varepsilon}\left(\bigcup_{i=j+1}^{n}\left\{z_{\sigma(i), 0}^{*}\right\}\right)=\varnothing .
$$

Observemos que a proposição resultará demonstrada se provarmos a seguinte condição mais forte:

Para cada $\delta \in(0, \varepsilon]$ existe $\eta_{\delta}>0$ de maneira que

$$
A_{j, \eta}(t) \subset \mathcal{O}_{\delta}\left(A_{j, 0}\right)
$$

e

$$
\bigcup_{i=j+1}^{n}\left\{\xi_{i, \eta}^{*}(t)\right\} \subset \mathcal{O}_{\delta}\left(\bigcup_{i=j+1}^{n}\left\{z_{\sigma(i), 0}^{*}\right\}\right)
$$

para todo $t \in \mathbb{R}, j=1,2, \cdots, n-1$ e $\eta \in\left[0, \eta_{\delta}\right]$.

Mas estas duas condições seguem, respectivamente, de (7.5.22) no Lema 7.5.4 e da hipótese $(c)$ e assim a proposição está demonstrada.

Agora estamos em posição de provar que as famílias $A_{j, \eta}$, de fato, constituem atratores locais, para $\eta$ suficientemente pequeno.

Proposição 7.5.6. Seja $X$ um espaço métrico e, para cada $\eta \in[0,1],\left\{T_{\eta}(t, s): t \geq s\right\}$ um processo de evolução em $X$ possuindo atrator pullback $\left\{\mathcal{A}_{\eta}(t): t \in \mathbb{R}\right\}$ satisfazendo as hipóteses de $(a)$ a (e) do Teorema 7.5.1.

Seja $\sigma: I_{n} \rightarrow I_{n}$ uma permutação tal que a n-upla ordenada

$$
\mathbf{D}_{0}=\left(z_{\sigma(1), 0}^{*}, z_{\sigma(2), 0}^{*}, \cdots, z_{\sigma(n), 0}^{*}\right)
$$

é uma decomposição de Morse para o atrator global $\mathcal{A}_{0}$ do semigrupo limite $\{S(t): t \geq$ 0\} e consideremos a n-upla ordenada

$$
\mathbf{D}_{\eta}=\left(\xi_{\sigma(1), \eta}^{*}, \xi_{\sigma(2), \eta}^{*}, \cdots, \xi_{\sigma(n), \eta}^{*}\right)
$$

Então, existe $\eta_{0}>0$ tal que a família invariante $A_{j, \eta}:=\left\{A_{j, \eta}(t): t \in \mathbb{R}\right\}$, definida em (7.5.20), é um atrator local para o processo de evolução $\left\{T_{\eta}(t, s): t \geq s\right\}$, para todo $j=1,2, \cdots, n$ e $\eta \in\left(0, \eta_{0}\right]$. 
Demonstração: Com efeito, do Lema 7.5.4 e da prova da Proposição 7.5.5, segue a existência de $\varepsilon>0, \delta \in(0, \varepsilon]$ e $\eta_{0}>0$ de modo que

$$
A_{j, \eta}(t) \subset \mathcal{O}_{\delta}\left(A_{j, 0}\right)
$$

e

$$
\bigcup_{i=j+1}^{n}\left\{\xi_{\sigma(i), \eta}^{*}(t)\right\} \subset \mathcal{O}_{\delta}\left(\bigcup_{i=j+1}^{n}\left\{z_{\sigma(i), 0}^{*}\right\}\right)
$$

para todo $j=1,2, \cdots, n-1, t \in \mathbb{R}$ e $\eta \in\left[0, \eta_{0}\right]$, onde $\varepsilon>0$ é tal que para todo $j=1,2, \cdots, n-1$

$$
\mathcal{O}_{2 \varepsilon}\left(A_{j, 0}\right) \cap \mathcal{O}_{2 \varepsilon}\left(\bigcup_{i=j+1}^{n}\left\{z_{\sigma(i), 0}^{*}\right\}\right)=\varnothing
$$

Agora, fixando $\eta \in\left[0, \eta_{0}\right]$, seja $\xi_{\eta}: \mathbb{R} \rightarrow X$ uma solução global para $\left\{T_{\eta}(t, s): t \geq s\right\}$ $\operatorname{com} \xi_{\eta}(t) \in \mathcal{O}_{\varepsilon}\left(A_{j, \eta}(t)\right)$ para todo $t \in \mathbb{R}$ e, recordando que $\left\{T_{\eta}(t, s): t \geq s\right\}$ é um processo de evolução de tipo gradiente com respeito a $\mathcal{E}_{\eta}=\left\{\xi_{1, \eta}^{*}, \cdots, \xi_{n, \eta}^{*}\right\}$ para cada $\eta \in\left[0, \eta_{0}\right]$ (isto graças ao Teorema 7.5.1), seja $i \in\{1,2, \cdots, n\}$ tal que

$$
\lim _{t \rightarrow-\infty} d\left(\xi_{\eta}(t), \xi_{\sigma(i), \eta}^{*}(t)\right)=0
$$

Então, por (7.5.33), necessariamente $i \leq j$, donde $\xi_{\eta}(t) \in W^{u}\left(\xi_{\sigma(i), \eta}^{*}\right)(t) \subset A_{j, \eta}(t)$ para todo $t \in \mathbb{R}$, o que nos diz que a família $A_{j, \eta}=\left\{A_{j, \eta}(t): t \in \mathbb{R}\right\}$ é invariante isolada para cada $j=1,2, \cdots, n$ e $\eta \in\left[0, \eta_{0}\right]$.

Por outro lado, seja $\xi_{\eta}: \mathbb{R} \rightarrow X$ uma solução global de $\left\{T_{\eta}(t, s): t \geq s\right\}$ com $\eta \in\left[0, \eta_{0}\right]$ e $\lim _{t \rightarrow-\infty} d\left(\xi_{\eta}(t), A_{j, \eta}(t)\right)=0$. Se $t_{0}$ é tal que $d\left(\xi_{\eta}(t), A_{j, \eta}(t)\right)<\varepsilon$ para todo $t \leq t_{0}$, como $\mathcal{O}_{\varepsilon}\left(A_{j, \eta}(t)\right) \cap \mathcal{O}_{\varepsilon}\left(\bigcup_{i=j+1}^{n}\left\{\xi_{\sigma(i), \eta}^{*}(t)\right\}\right)=\varnothing$ para todo $t \in \mathbb{R}$, usando o mesmo raciocínio que acabamos de usar acima, conclui-se que $\xi_{\eta}(t) \in A_{j, \eta}(t)$ para todo $t \in \mathbb{R}$, ou seja, $W^{u}\left(A_{j, \eta}\right)(t) \subset A_{j, \eta}(t)$ para cada $t \in \mathbb{R}$. Agora, a inclusão $A_{j, \eta}(t) \subset W^{u}\left(A_{j, \eta}\right)(t)$, para cada $t \in \mathbb{R}$, é válida simplesmente pela invariância da família $A_{j, \eta}$, mostrando que $W^{u}\left(A_{j, \eta}\right)(t)=A_{j, \eta}(t)$ para todo $t \in \mathbb{R}$ e completando a prova da proposição.

Podemos agora demonstrar que a $n$-upla ordenada $\mathbf{D}_{\eta}=\left(\xi_{\sigma(1), \eta}^{*}, \xi_{\sigma(2), \eta}^{*}, \cdots, \xi_{\sigma(n), \eta}^{*}\right)$, que foi obtida por meio de uma permutação $\sigma: I_{n} \rightarrow I_{n}$ que faz com que a $n$-upla $\mathbf{D}_{0}=\left(z_{\sigma(1), 0}^{*}, z_{\sigma(2), 0}^{*}, \cdots, z_{\sigma(n), 0}^{*}\right)$ de lugar a uma decomposição de Morse para o atrator global do semigrupo limite, define também uma decomposição de Morse do atrator pullback de $T_{\eta}(\cdot, \cdot)$ para $\eta>0$ suficientemente pequeno, o que é o conteúdo do 
Teorema 7.5.7. Seja $X$ um espaço métrico e, para cada $\eta \in[0,1],\left\{T_{\eta}(t, s): t \geq s\right\}$ um processo de evolução em $X$ possuindo atrator pullback $\left\{\mathcal{A}_{\eta}(t): t \in \mathbb{R}\right\}$ satisfazendo as hipóteses de $(a)$ a (e) do Teorema 7.5.1.

Seja $\sigma: I_{n} \rightarrow I_{n}$ uma permutação tal que a n-upla ordenada

$$
\mathbf{D}_{0}=\left(z_{\sigma(1), 0}^{*}, z_{\sigma(2), 0}^{*}, \cdots, z_{\sigma(n), 0}^{*}\right)
$$

é uma decomposição de Morse para o atrator global $\mathcal{A}_{0}$ do semigrupo limite $\{S(t): t \geq$ $0\}$ e consideremos a n-upla ordenada

$$
\mathbf{D}_{\eta}=\left(\xi_{\sigma(1), \eta}^{*}, \xi_{\sigma(2), \eta}^{*}, \cdots, \xi_{\sigma(n), \eta}^{*}\right)
$$

Consideremos também as famílias de atratores locais $A_{j, \eta}=\left\{A_{j, \eta}(t): t \in \mathbb{R}\right\}$ definidas em (7.5.20) e seus repulsores correspondentes $A_{j, \eta}^{*}=\left\{A_{j, \eta}^{*}(t): t \in \mathbb{R}\right\}$ dados em (7.5.21).

Então, existe $\eta_{0}>0$ tal que para cada $\eta \in\left[0, \eta_{0}\right], t \in \mathbb{R}$ e $j=1,2, \cdots, n$ verifica-se a igualdade

$$
A_{j, \eta}(t) \cap A_{j-1, \eta}^{*}(t)=\left\{\xi_{\sigma(j), \eta}^{*}(t)\right\}
$$

Em outras palavras, a n-upla ordenada $\mathbf{D}_{\eta}=\left(\xi_{\sigma(1), \eta}^{*}, \xi_{\sigma(2), \eta}^{*}, \cdots, \xi_{\sigma(n), \eta}^{*}\right)$ é uma decomposição de Morse do atrator pullback $\left\{\mathcal{A}_{\eta}(t): t \in \mathbb{R}\right\}$ de $\left\{T_{\eta}(t, s): t \geq s\right\}$ para todo $\eta \in\left[0, \eta_{0}\right]$. Em particular, tal decomposição de Morse se comporta continuamente.

Demonstração: Com efeito, é claro que $\xi_{\sigma(j), \eta}^{*}(t) \in W^{u}\left(\xi_{\sigma(j), \eta}^{*}\right)(t) \subset A_{j, \eta}(t)$ para todo $j=1,2, \cdots, n, t \in \mathbb{R}$ e $\eta \in[0,1]$. Agora, usando a Proposição 7.5.5, sejam $\varepsilon>0$ e $\eta_{0}>0$ tais que, para todo $t \in \mathbb{R}, j=2,3, \cdots, n$ e $\eta \in\left[0, \eta_{0}\right]$, temos

$$
\mathcal{O}_{\varepsilon}\left(A_{j-1, \eta}(t)\right) \cap \mathcal{O}_{\varepsilon}\left(\bigcup_{i=j}^{n}\left\{\xi_{\sigma(i), \eta}^{*}(t)\right\}\right)=\varnothing .
$$

Portanto, (7.5.37) nos diz que, $d\left(T_{\eta}(r+t, t) \xi_{\sigma(j), \eta}^{*}(t), A_{j-1, \eta}(t+r)\right)=d\left(\xi_{\sigma(j), \eta}^{*}(t+\right.$ $\left.r), A_{j-1, \eta}(t+r)\right) \geq \varepsilon$ sempre que $r \geq 0$. Donde $\xi_{\sigma(j), \eta}^{*}(t) \in A_{j-1, \eta}^{*}(t)$ para todo $t \in \mathbb{R}$ e $\operatorname{assim}\left\{\xi_{\sigma(j), \eta}^{*}(t)\right\} \subset A_{j, \eta}(t) \cap A_{j-1, \eta}^{*}(t)$ sempre que $\eta \in\left(0, \eta_{0}\right], t \in \mathbb{R}$ e $j=1,2, \cdots, n$.

Reciprocamente, observemos que, pelo Lema 7.5.4, dado $\delta \in(0, \varepsilon]$ existe $\eta_{\delta}>0$ de modo que para todo $\eta \in\left(0, \eta_{\delta}\right], t \in \mathbb{R}$ e $j=1,2, \cdots, n$ temos

$$
A_{j, \eta}(t) \cap A_{j-1, \eta}^{*}(t) \subset \mathcal{O}_{\frac{\delta}{2}}\left(A_{j, 0}\right) \cap \mathcal{O}_{\frac{\delta}{2}}\left(A_{j-1,0}^{*}\right)
$$

Agora, afirmamos que a seguinte condição se verifica:

$A_{j, \eta}(t) \cap A_{j-1, \eta}^{*}(t) \subset\left\{\xi_{\sigma(j), \eta}^{*}(t)\right\}$ para todo $t \in \mathbb{R}, j=1,2, \cdots, n$ e todo $\eta>0$ suficientemente pequeno. 
Porque, caso contrário, existiriam $j=1,2, \cdots, n$, uma sequência $\left(\eta_{k}\right)_{k \in \mathbb{N}}$ em $(0,1]$ com $\eta_{k} \rightarrow 0^{+}$, uma sequência $\left(t_{k}\right)_{k \in \mathbb{N}}$ em $\mathbb{R}$ e uma sequência $\left(x_{k}\right)_{k \in \mathbb{N}}$ de pontos de $X \operatorname{com} x_{k} \in A_{j, \eta_{k}}\left(t_{k}\right) \cap A_{j-1, \eta_{k}}^{*}\left(t_{k}\right)$ mas $x_{k} \neq \xi_{\sigma(j), \eta_{k}}^{*}\left(t_{k}\right)$ para todo $k \in \mathbb{N}$. Por isso, para cada $k \in \mathbb{N}$, existiria uma solução global $\xi_{k}: \mathbb{R} \rightarrow X$ de $\left\{T_{\eta_{k}}(t, s): t \geq s\right\}$ com $\xi_{k}\left(t_{k}\right)=x_{k}$ para a qual, sem perda de generalidade podemos supor que, existem índices fixos $i \leq j$ e $l \geq j$ com

$$
\lim _{t \rightarrow-\infty} d\left(\xi_{k}(t), \xi_{\sigma(i), \eta_{k}}^{*}(t)\right)=0 \text { e } \lim _{t \rightarrow \infty} d\left(\xi_{k}(t), \xi_{\sigma(l), \eta_{k}}^{*}(t)\right)=0 .
$$

Além disso, por (7.5.38), $\xi_{k}(t) \in \mathcal{O}_{\frac{\delta}{2}}\left(A_{j, 0}\right) \cap \mathcal{O}_{\frac{\delta}{2}}\left(A_{j-1,0}^{*}\right)$ para todo $k \in \mathbb{N}$ e $t \in \mathbb{R}$. Portanto (confira a prova do Lema 7.5.4), se escolhemos, para $k$ suficientemente grande, $\tau_{k}$ e $\sigma_{k} \mathrm{com}$

$$
d\left(\xi_{k}(t), z_{\sigma(i), 0}^{*}\right)<\frac{\delta}{2} \text { para } t \leq \tau_{k} \text { e } d\left(\xi_{k}(t), z_{\sigma(l), 0}^{*}\right)<\frac{\delta}{2} \text { para } t \geq \sigma_{k},
$$

daí, necessariamente, teríamos $z_{\sigma(i), 0}^{*}$ e $z_{\sigma(l), 0}^{*}$ em $\mathcal{O}_{\delta}\left(A_{j, 0}\right) \cap \mathcal{O}_{\delta}\left(A_{j-1,0}^{*}\right)$ então, por (7.5.37), $i=l=j$, ou seja, $z_{\sigma(i), 0}^{*}=z_{\sigma(l), 0}^{*}=z_{\sigma(j), 0}^{*}$, o que nos estaria dizendo que a solução $\xi_{k}: \mathbb{R} \rightarrow X$ é uma solução homoclínica (porque $x_{k} \neq \xi_{j, \eta_{k}}^{*}\left(t_{k}\right)$ para todo $k)$, contradizendo portanto o fato de que $\left\{T_{\eta_{k}}(t, s): t \geq s\right\}$ é de tipo gradiente com respeito a $\mathcal{E}_{\eta_{k}}=\left\{\xi_{1, \eta_{k}}^{*}, \cdots, \xi_{n, \eta_{k}}^{*}\right\}$, completando a prova.

Os resultados que estabelecemos a seguir são aqueles necessários à construção da função de Lyapunov para um par atrator-repulsor não autônomo.

O primeiro que provamos é o fato de que, para os atratores locais que construímos acima, verifica-se a propriedade de separação entre o atrator local e seu repulsor complementar, hipótese que foi exigida em (7.4.12) na Proposição 7.4.3.

Proposição 7.5.8. Seja $X$ um espaço métrico e, para cada $\eta \in[0,1],\left\{T_{\eta}(t, s): t \geq s\right\}$ um processo de evolução em $X$ possuindo atrator pullback $\left\{\mathcal{A}_{\eta}(t): t \in \mathbb{R}\right\}$ e satisfazendo as condiciones de (a) a (e) do Teorema 7.5.1.

Seja também, $\sigma: I_{n} \rightarrow I_{n}$ uma permutação tal que a n-upla ordenada

$$
\mathbf{D}_{0}=\left(z_{\sigma(1), 0}^{*}, z_{\sigma(2), 0}^{*}, \cdots, z_{\sigma(n), 0}^{*}\right)
$$

define uma decomposição de Morse para o atrator global $\mathcal{A}_{0}$ do semigrupo limite $\{S(t)$ : $t \geq 0\}$.

Consideremos as familias de atratores locais e seus correspondentes repulsores definidos em (7.5.20) e em (7.5.21).

Então, existem $\varepsilon>0$ e $\eta_{0}>0$ tais que, para todo $t \in \mathbb{R}, j=1,2, \cdots, n$ e $\eta \in\left[0, \eta_{0}\right]$, tem-se

$$
\mathcal{O}_{\varepsilon}\left(A_{j, \eta}(t)\right) \cap \mathcal{O}_{\varepsilon}\left(A_{j, \eta}^{*}(t)\right)=\varnothing
$$


Demonstração: Com efeito, pelo fato de que os conjuntos $A_{j, 0}$ e $A_{j, 0}^{*}$ são compactos disjuntos para todo $j$, podemos escolher $\varepsilon>0$ de maneira que para todo $j=1,2, \cdots, n$

$$
\mathcal{O}_{2 \varepsilon}\left(A_{j, 0}\right) \cap \mathcal{O}_{2 \varepsilon}\left(A_{j, 0}^{*}\right)=\varnothing
$$

E simplesmente pelo fato de que as famílias $A_{j, \eta}$ e $A_{j, \eta}^{*}$ são semicontínuas superiormente (Lema 7.5.22), para cada $\delta \in(0, \varepsilon]$, existe $\eta_{\delta}>0$ tal que

$$
A_{j, \eta}(t) \subset \mathcal{O}_{\delta}\left(A_{j, 0}\right)
$$

$\mathrm{e}$

$$
A_{j, \eta}^{*}(t) \subset \mathcal{O}_{\delta}\left(A_{j, 0}^{*}\right),
$$

para todo $\eta \in\left[0, \eta_{\delta}\right], t$ real e $j=1,2, \cdots, n$ e o resultado segue imediatamente.

Nesta etapa queremos demonstrar que se encontra satisfeita a conclusão do Lema 7.3.3, para os atratores locais que aqui construímos para as perturbações não autônomas de um semigrupo gradiente. O seguinte resultado desempenhará um papel fundamental nesta tarefa.

Lema 7.5.9. Seja $\left\{T_{\eta}(t, s): t \geq s\right\}_{\eta \in[0,1]}$ uma família coletivamente assintoticamente compacta de processos de evolução em um espaço métrico $X$ tal que, para cada $\eta \in[0,1]$, $T_{\eta}(\cdot, \cdot)$ possui atrator pullback $\mathcal{A}_{\eta}:=\left\{\mathcal{A}_{\eta}(t): t \in \mathbb{R}\right\}$ e que, além disso, as condições (a) e $(d)$ do Teorema 7.5.1 estejam satisfeitas.

Suponhamos que $T_{0}(t, s)=S(t-s)$, para todo $t \geq s$, onde $\{S(t): t \geq 0\}$ é um semigrupo.

Seja também, para cada $\eta \in[0,1], A_{\eta}:=\left\{A_{\eta}(t): t \in \mathbb{R}\right\}$ tal que $A_{\eta}(t) \subset \mathcal{A}_{\eta}(t)$ para todo $t \in \mathbb{R}$ com $A_{0}$ sendo um atrator local para $\{S(t): t \geq 0\}$ (isto é, $\omega\left(\mathcal{O}_{\varepsilon}\left(A_{0}\right)\right)=A_{0}$ para algum $\varepsilon>0)$.

Se a familia $\left(A_{\eta}\right)_{\eta \in[0,1]}$ é contínua em $\eta=0$, ou seja, se

$$
\lim _{\eta \rightarrow 0^{+}} \sup _{t \in \mathbb{R}} d_{H}\left(A_{\eta}(t), A_{0}\right)=0
$$

então, dado $\delta \in(0, \varepsilon)$ existem $\delta^{\prime} \in(0, \delta)$ e $\eta_{\delta}>0$ tais que para todo $\eta \in\left[0, \eta_{\delta}\right]$ tem-se

$$
T_{\eta}(t, s)\left(\mathcal{O}_{\delta^{\prime}}\left(A_{\eta}(s)\right)\right) \subset \mathcal{O}_{\delta}\left(A_{\eta}(t)\right) \text { toda vez que } t \geq s .
$$

Demonstração: Suponhamos que esta conclusão não se cumpra, então podemos encontrar $\delta>0$ e sequências $\left(x_{j}\right)_{j \in \mathbb{N}}$ em $X,\left(\eta_{j}\right)_{j \in \mathbb{N}}$ em $(0,1]$ e $\left(t_{j}\right)_{j \in \mathbb{N}}$ e $\left(\tau_{j}\right)_{j \in \mathbb{N}}$ em $\mathbb{R}$ tais que $\eta_{j} \underset{j \rightarrow \infty}{\rightarrow} 0^{+}, t_{j} \underset{j \rightarrow \infty}{\rightarrow} \infty, d\left(x_{j}, A_{\eta_{j}}\left(\tau_{j}\right)\right)<\frac{1}{j}$ para todo $j$,

$$
d\left(T_{\eta_{j}}\left(t+\tau_{j}, \tau_{j}\right) x_{j}, A_{\eta_{j}}\left(t+\tau_{j}\right)\right)<\delta \text { para todo } t \in\left[0, t_{j}\right) \text { e todo } j \in \mathbb{N}
$$


$\mathrm{e}$

$$
d\left(T_{\eta_{j}}\left(t_{j}+\tau_{j}, \tau_{j}\right) x_{j}, A_{\eta_{j}}\left(t_{j}+\tau_{j}\right)\right)=\delta \text { para todo } j \in \mathbb{N} .
$$

Se, para cada natural $j$, definimos $\zeta_{j}:\left[-t_{j}, \infty\right) \rightarrow X$ por $\zeta_{j}(t):=T_{\eta_{j}}\left(t+t_{j}+\tau_{j}, \tau_{j}\right) x_{j}$, $t \in\left[-t_{j}, \infty\right)$. Do item $(i i)$ do Lema 7.5.3 segue a existência de uma solução global limitada, $\zeta: \mathbb{R} \rightarrow X$ de $\{S(t): t \geq 0\}$ e uma subsequência de $\left(\zeta_{j}\right)_{j \in \mathbb{N}}$, indicada com a mesma notação, tal que para todo real $t, \zeta(t)=\lim _{j \rightarrow \infty} \zeta_{j}(t)$.

Por outro lado, dado $t<0$, para $j$ suficientemente grande

$$
d\left(\zeta_{j}(t), A_{0}\right) \leq d\left(\zeta_{j}(t), A_{\eta_{j}}\left(t+\tau_{j}\right)\right)+\operatorname{dist}\left(A_{\eta_{j}}\left(t+\tau_{j}\right), A_{0}\right),
$$

donde, pela semicontinuidade superior de $\left(A_{\eta}\right)_{\eta \in[0,1]}$, obtém-se que para todo $t<0$

$$
d\left(\zeta(t), A_{0}\right) \leq \delta
$$

e pelo fato de que $\delta=d\left(\zeta_{j}(0), A_{\eta_{j}}\left(t_{j}+\tau_{j}\right)\right) \leq d\left(\zeta_{j}(0), A_{0}\right)+\operatorname{dist}\left(A_{0}, A_{\eta_{j}}\left(t_{j}+\tau_{j}\right)\right)$, da semicontinuidade inferior de $\left(A_{\eta}\right)_{\eta \in[0,1]}$, conclui-se que $d\left(\zeta(0), A_{0}\right)=\delta$.

Mas, como $\delta<\varepsilon, A_{0}$ deveria atrair $K:=\{\zeta(t): t \leq 0\}$, o que contradiz o fato de que $d\left(\zeta(0), A_{0}\right)=\delta$ e termina a prova.

A próxima proposição nos garante que as perturbações pequenas de um semigrupo de tipo gradiente, satisfazem a conclusão do Lema 7.3.3, considerando-se vizinhanças dos atratores locais em $X$, mais precisamente temos:

Proposição 7.5.10. Seja $X$ um espaço métrico e, para cada $\eta \in[0,1],\left\{T_{\eta}(t, s)\right.$ : $t \geq s\}$ um processo de evolução em $X$ possuindo atrator pullback $\left\{\mathcal{A}_{\eta}(t): t \in \mathbb{R}\right\}$, satisfazendo as condições de $(a)$ a $(e)$ do Teorema 7.5.1 e, além disso, suponha que a familia $\left\{T_{\eta}(t, s): t \geq s\right\}_{\eta \in[0,1]}$ seja coletivamente assintoticamente compacta.

Seja também, $\sigma: I_{n} \rightarrow I_{n}$ uma permutação tal que a $n$-upla ordenada

$$
\mathbf{D}_{0}=\left(z_{\sigma(1), 0}^{*}, z_{\sigma(2), 0}^{*}, \cdots, z_{\sigma(n), 0}^{*}\right)
$$

é uma decomposição de Morse para o atrator global $\mathcal{A}_{0}$ do semigrupo limite $\{S(t): t \geq$ $0\}$.

Consideremos as famílias de atratores locais $A_{j, \eta}$ definidas como antes e suponhamos que as mesmas se comportem continuamente, isto é, que para todo $j=1,2, \cdots, n$

$$
\lim _{\eta \rightarrow 0^{+}} \sup _{t \in \mathbb{R}} d_{H}\left(A_{j, \eta}(t), A_{j, 0}\right)=0 .
$$

Então, existe $\eta_{0}>0$ tal que para todo $\eta \in\left[0, \eta_{0}\right]$ e todo $j=1,2, \cdots$, n o atrator local $A_{j, \eta}=\left\{A_{j, \eta}(t): t \in \mathbb{R}\right\}$ satisfaz a conclusão do Lema 7.3.3 considerando-se vizinhanças de $A_{j, \eta}$ em $X$ (confira a hipótese (a) da Proposição 7.4.4). 
Demonstração: Com efeito, fixemos $\delta_{1} \in(0, \varepsilon), \eta_{1}=\eta_{1}\left(\delta_{1}\right)>0$ e $\delta_{1}^{\prime}=\delta_{1}^{\prime}\left(\delta_{1}\right) \in$ $\left(0, \delta_{1}\right)$ tais que a conclusão do Lema 7.5 .9 se verifica, ou seja, para todo $\eta \in\left[0, \eta_{1}\right]$ e todo $j=1,2, \cdots, n$ temos

$$
T_{\eta}(t, s)\left(\mathcal{O}_{\delta_{1}^{\prime}}\left(A_{j, \eta}(s)\right)\right) \subset \mathcal{O}_{\delta_{1}}\left(A_{j, \eta}(t)\right) \text { sempre que } t \geq s
$$

onde $\varepsilon>0$ é tal que para todo $j, \omega\left(\mathcal{O}_{\varepsilon}\left(A_{j, 0}\right)\right)=A_{j, 0}$.

Por outro lado, pela hipótese de continuidade das famílias $\left(A_{j, \eta}\right)_{\eta \in[0,1]}$ podemos escolher $0<\delta_{3}^{\prime}<\delta_{2}^{\prime}<\delta_{1}^{\prime}$ e $\eta_{2} \in\left(0, \eta_{1}\right]$ tais que, para todo $\eta \in\left[0, \eta_{2}\right], j=1,2, \cdots, n$ e $s \in \mathbb{R}$, verificam-se as inclusões

$$
\mathcal{O}_{\delta_{3}^{\prime}}\left(A_{j, \eta}(s)\right) \subset \mathcal{O}_{\delta_{2}^{\prime}}\left(A_{j, 0}\right) \subset \mathcal{O}_{\delta_{1}^{\prime}}\left(A_{j, \eta}(s)\right)
$$

Portanto, levando em conta $(7.5 .41), \omega_{\eta}\left(\mathcal{O}_{\delta_{2}^{\prime}}\left(A_{j, 0}\right) ; s\right)^{3} \subset \overline{\mathcal{O}_{\delta_{1}}\left(A_{j, \eta}(s)\right)}$ para todo real $s$, todo $\eta \in\left[0, \eta_{2}\right]$ e $j=1,2, \cdots, n$.

Segue-se do fato de que cada $A_{j, \eta}$ é invariante isolado (porque cada um deles é atrator local, segundo a Proposição 7.5.6, $\operatorname{com} \varepsilon>0$ sendo um raio de vizinhança onde a maximalidade da família $A_{j, \eta}$ verifica-se para todo $j$ e $\eta$ suficientemente pequeno) que $\omega_{\eta}\left(\mathcal{O}_{\delta_{2}^{\prime}}\left(A_{j, 0}\right) ; s\right)$ está contido em $A_{j, \eta}(s)$ para todo $s \in \mathbb{R}$ e $\eta \in\left[0, \eta_{2}\right]$.

Agora, dados $t \in \mathbb{R}$ e $\eta \in\left[0, \eta_{2}\right], \omega_{\eta}\left(\mathcal{O}_{\delta_{2}^{\prime}}\left(A_{j, 0}\right) ; t\right)$ atrai, em sentido pullback e no instante $t$, o conjunto $\mathcal{O}_{\delta_{2}^{\prime}}\left(A_{j, 0}\right)$, então dado $\delta>0$ existe $\tau_{(\delta, t)}>0$ de modo que

$$
T_{\eta}(t, t-\tau) \mathcal{O}_{\delta_{2}^{\prime}}\left(A_{j, 0}\right) \subset \mathcal{O}_{\delta}\left(\omega_{\eta}\left(\mathcal{O}_{\delta_{2}^{\prime}}\left(A_{j, 0}\right) ; t\right)\right),
$$

para todo $\tau \geq \tau_{(\delta, t)}$.

E daí segue-se, graças à cadeia de inclusões $(7.5 .42)$ e ao fato de que $\omega_{\eta}\left(\mathcal{O}_{\delta_{2}^{\prime}}\left(A_{j, 0}\right) ; s\right) \subset$ $A_{j, \eta}(s)$ para todo $s \in \mathbb{R}$, que

$$
T_{\eta}(t, t-\tau) \mathcal{O}_{\delta_{3}^{\prime}}\left(A_{j, \eta}(t-\tau)\right) \subset \mathcal{O}_{\delta}\left(A_{j, \eta}(t)\right)
$$

para todo $\tau \geq \tau_{(\delta, t)}$.

Finalmente, pela continuidade do processo $T_{\eta}(\cdot, \cdot)$, podemos escolher $\delta^{\prime} \in\left(0, \min \left\{\delta, \delta_{3}^{\prime}\right\}\right)$ tal que

$$
T_{\eta}(t, t-\tau) \mathcal{O}_{\delta^{\prime}}\left(A_{j, \eta}(t-\tau)\right) \subset \mathcal{O}_{\delta}\left(A_{j, \eta}(t)\right)
$$

para todo $\tau \in\left[0, \tau_{(\delta, t)}\right]$, o que completa a prova da proposição fazendo-se $\eta_{0}:=\eta_{2}$.

\footnotetext{
${ }^{3}$ Dado um real $s$, o símbolo $\omega_{\eta}\left(\mathcal{O}_{\delta_{2}^{\prime}}\left(A_{j, 0}\right) ; s\right)$ indica o conjunto $\omega$-limite do conjunto $\mathcal{O}_{\delta_{2}^{\prime}}\left(A_{j, 0}\right)$ em sentido pullback e em tempo $s$ com respeito ao processo de evolução $\left\{T_{\eta}(t, s): t \geq s\right\}$. É um resultado conhecido, que o conjunto $\omega_{\eta}\left(\mathcal{O}_{\delta_{2}^{\prime}}\left(A_{j, 0}\right) ; s\right)$ atrai o conjunto $\mathcal{O}_{\delta_{2}^{\prime}}\left(A_{j, 0}\right)$ em sentido pullback e em tempo $s(\operatorname{veja}[7])$.
} 
Finalmente, podemos concluir que a classe das perturbações não autônomas pequenas de semigrupos gradientes satisfaz todas as hipóteses que impusemos para a construção da decomposição de Morse para um processo de evolução de tipo gradiente bem como as hipóteses necessárias para se construir as funções de Lyapunov dos pares atrator-repulsor.

Resumimos estes fatos no seguinte:

Teorema 7.5.11. Seja $X$ um espaço métrico e, para cada $\eta \in[0,1],\left\{T_{\eta}(t, s): t \geq s\right\}$ um processo de evolução em $X$ possuindo atrator pullback $\left\{\mathcal{A}_{\eta}(t): t \in \mathbb{R}\right\}$, satisfazendo as condições de (a) a (e) do Teorema 7.5.1 e que, além disso, a familia $\left\{T_{\eta}(t, s): t \geq\right.$ $s\}_{\eta \in[0,1]}$ seja coletivamente assintoticamente compacta.

Seja também, $\sigma: I_{n} \rightarrow I_{n}$ uma permutação tal que a $n$-upla ordenada

$$
\mathbf{D}_{0}=\left(z_{\sigma(1), 0}^{*}, z_{\sigma(2), 0}^{*}, \cdots, z_{\sigma(n), 0}^{*}\right)
$$

é uma decomposição de Morse para o atrator global $\mathcal{A}_{0}$ do semigrupo limite $\{S(t): t \geq$ $0\}$.

Então, existe $\eta_{0}>0$ tal que para todo $\eta \in\left[0, \eta_{0}\right]$ a $n$-upla ordenada

$$
\mathbf{D}_{\eta}=\left(\xi_{\sigma(1), \eta}^{*}, \xi_{\sigma(2), \eta}^{*}, \cdots, \xi_{\sigma(n), \eta}^{*}\right)
$$

é uma decomposição de Morse para o atrator pullback de $\left\{T_{\eta}(t, s): t \geq s\right\}$.

Nestas condições, se para cada $j=1,2, \cdots, n$ a família de atratores locais $\left(A_{j, \eta}(\cdot)\right)_{\eta \in[0,1]}$ se comporta continuamente no sentido da proposição anterior, então para cada $\eta \in$ $\left[0, \eta_{0}\right],\left\{T_{\eta}(t, s): t \geq s\right\}$ é um processo de evolução gradiente com respeito ao conjunto $\mathcal{E}_{\eta}=\left\{\xi_{1, \eta}^{*}, \cdots, \xi_{n, \eta}^{*}\right\}$, de soluções globais isoladas, no sentido da Definição 7.4.1.

Além disso, se para cada $j=1,2, \cdots, n$ a família de atratores pullback $\left(\mathcal{A}_{j, \eta}(\cdot)\right)_{\eta \in[0,1]}$ cumpre as condições do Lema 7.4.5, entãs as função de Lyapunov $V_{\eta}: \mathbb{R} \times X \rightarrow \mathbb{R}$ dos porcessos $\left(T_{\eta}(\cdot)\right)_{\eta \in[0,1]}$ sãoo contínua em ambas as variáveis com $V_{\eta}\left(t, \xi_{\sigma(i), \eta}^{*}(t)\right)=i$, para todo $t \in \mathbb{R}$ e $i=1, \cdots, n$.

\subsubsection{Convergência das funções de Lyapunov}

Aqui, simplesmente para completar a análise sobre perturbações não autônomas de um semigrupo gradiente, seguindo as idéias desenvolvidas na Seção 5.2, damos condições suficientes para garantir a convergência das funções de Lyapunov dos processos perturbados, em um sentido a ser precisado no próximo teorema, para a função de Lyapunov do semigrupo limite. 
Teorema 7.5.12. Seja $\left\{T_{\eta}(t, s): t \geq s\right\}_{\eta \in[0,1]}$ uma família coletivamente assintoticamente compacta de processos de evolução em um espaço métrico $X$ com correspondente família de atratores pullback $\left(\mathcal{A}_{\eta}\right)_{\eta \in[0,1]}$, satisfazendo as condições $(a)$ e $(d)$ do Teorema 7.5.1.

Suponhamos que $T_{0}(t, s)=S(t-s)$ sempre que $t \geq s$, para um certo semigrupo $\{S(t): t \geq 0\}$

Para cada $\eta \in[0,1]$, seja $A_{\eta}:=\left\{A_{\eta}(t): t \in \mathbb{R}\right\}$ um atrator local para $\left\{T_{\eta}(t, s)\right.$ : $t \geq s\}$ com correspondente repulsor complementar $A_{\eta}^{*}:=\left\{A_{\eta}^{*}(t): t \in \mathbb{R}\right\}$ de modo que $A_{0}(t)=A_{0}$ para todo real $t$, onde $A_{0}$ é um atrator local para o semigrupo $\{S(t): t \geq 0\}$.

Suponhamos também, que as famílias $\left(A_{\eta}\right)_{\eta \in[0,1]},\left(A_{\eta}^{*}\right)_{\eta \in[0,1]}$ e $\left(\mathcal{A}_{\eta}\right)_{\eta \in[0,1]}$ sejam contínuas em $\eta=0$, isto é, que se cumpram as convergências

$$
\begin{aligned}
& \lim _{\eta \rightarrow 0^{+}} \sup _{t \in \mathbb{R}} d_{H}\left(A_{\eta}(t), A_{0}\right)=0 \\
& \lim _{\eta \rightarrow 0^{+}} \sup _{t \in \mathbb{R}} d_{H}\left(A_{\eta}^{*}(t), A_{0}^{*}\right)=0
\end{aligned}
$$

$e$

$$
\lim _{\eta \rightarrow 0^{+}} \sup _{t \in \mathbb{R}} d_{H}\left(\mathcal{A}_{\eta}(t), \mathcal{A}_{0}\right)=0
$$

Finalmente, para cada $\eta \in[0,1]$, seja $f_{\eta}: \mathbb{R} \times X \rightarrow \mathbb{R}$ a função de Lyapunov associada ao par atrator-repulsor $\left(A_{\eta}, A_{\eta}^{*}\right)$ definida, como antes, por

$$
f_{\eta}(t, x):=k_{\eta}(t, x)+h_{\eta}(t, x),(t, x) \in \mathbb{R} \times X
$$

onde

$$
h_{\eta}(t, x):=\sup _{r \geq 0} d\left(T_{\eta}(r+t, t) x, \mathcal{A}_{\eta}(t+r)\right),(t, x) \in \mathbb{R} \times X
$$

$e$

$k_{\eta}(t, x):=\sup _{r \geq 0} l_{\eta}(t+r, T(t+r, t) x)$, sendo $l_{\eta}(t, x):=\frac{d\left(x, A_{\eta}(t)\right)}{d\left(x, A_{\eta}(t)\right)+d\left(x, A_{\eta}^{*}(t)\right)},(t, x) \in \mathbb{R} \times X$.

Então, para cado compacto $K$ de $X$ tem-se

$$
\lim _{\eta \rightarrow 0^{+}} \sup _{t \in \mathbb{R}} \sup _{x \in K}\left|f_{\eta}(t, x)-f_{0}(x)\right|=0 .
$$

Demonstração: Como fizemos no caso autônomo, dividimos a demonstração em três passos:

Passo 1: Convergência uniforme das $l_{\eta}$ para $l_{0}$ sobre todo o produto $\mathbb{R} \times X$, ou seja,

$$
\lim _{\eta \rightarrow 0^{+}} \sup _{t \in \mathbb{R}} \sup _{x \in X}\left|l_{\eta}(t, x)-l_{0}(x)\right|=0 .
$$


Com efeito, pela desigualdade triangular da semidistância de Hausdorff, para cada $\eta \in[0,1], x \in X$ e $t \in \mathbb{R}$ temos

$$
\left|d\left(x, A_{\eta}(t)\right)-d\left(x, A_{0}\right)\right| \leq d_{H}\left(A_{\eta}(t), A_{0}\right)
$$

e

$$
\left|d\left(x, A_{\eta}^{*}(t)\right)-d\left(x, A_{0}^{*}\right)\right| \leq d_{H}\left(A_{\eta}^{*}(t), A_{0}^{*}\right) .
$$

Agora, dados $\eta \in[0,1], t \in \mathbb{R}$ e $x \in X$ tem-se a seguinte igualdade

$$
l_{\eta}(t, x)-l_{0}(x)=\frac{\left[d\left(x, A_{\eta}(t)\right)-d\left(x, A_{0}\right)\right] d\left(x, A_{0}^{*}\right)+d\left(x, A_{0}\right)\left[d\left(x, A_{0}^{*}\right)-d\left(x, A_{\eta}^{*}(t)\right)\right]}{\left[d\left(x, A_{\eta}(t)\right)+d\left(x, A_{\eta}^{*}(t)\right)\right]\left[d\left(x, A_{0}\right)+d\left(x, A_{0}^{*}\right)\right]} .
$$

Como $d\left(A_{0}, A_{0}^{*}\right)>\mu$ para algum $\mu>0$, usando o fato de que as famílias de atratores e repulsores são contínuas, existe $\tilde{\eta} \in(0,1]$ tal que $d\left(A_{\eta}(t), A_{\eta}^{*}(t)\right) \geq \frac{\mu}{2}$, para todo $\eta \in[0, \tilde{\eta}]$ e todo $t \in \mathbb{R}$ (observemos que, daí, segue-se também que as funções $l_{\eta}$ estão bem definidas para $\eta$ suficientemente pequeno). De (7.5.43) e (7.5.44) obtém-se, levando em conta a última igualdade, que

$$
\begin{aligned}
\left|l_{\eta}(t, x)-l_{0}(x)\right| & \leq \frac{1}{d\left(x, A_{\eta}(t)\right)+d\left(x, A_{\eta}^{*}(t)\right)}\left[d_{H}\left(A_{\eta}(t), A_{0}\right)+d_{H}\left(A_{\eta}^{*}(t), A_{0}^{*}\right)\right] \\
& \leq \frac{1}{\mu}\left[d_{H}\left(A_{\eta}(t), A_{0}\right)+d_{H}\left(A_{\eta}^{*}(t), A_{0}^{*}\right)\right]
\end{aligned}
$$

para todo $t \in \mathbb{R}, x \in X$ e $\eta \in[0, \tilde{\eta}]$ e, portanto, para $\eta \in[0, \tilde{\eta}]$ temos

$$
\sup _{t \in \mathbb{R}} \sup _{x \in X}\left|l_{\eta}(t, x)-l_{0}(x)\right| \leq \frac{1}{\mu}\left[\sup _{t \in \mathbb{R}} d_{H}\left(A_{\eta}(t), A_{0}\right)+\sup _{t \in \mathbb{R}} d_{H}\left(A_{\eta}^{*}(t), A_{0}^{*}\right)\right] .
$$

Donde conclui-se a convergência uniforme, em $\mathbb{R} \times X$, simplesmente usando a hipótese de continuidade das famílias de atratores e seus repulsores.

Passo 2: Para cada compacto $K \subset X$ temos

$$
\lim _{\eta \rightarrow 0^{+}} \sup _{t \in \mathbb{R}} \sup _{x \in K}\left|k_{\eta}(t, x)-k_{0}(x)\right|=0 .
$$

Com efeito, dado $x \in X$, pelo item (ii) do Lema 4.1.7, temos $S(t) x \underset{t \rightarrow \infty}{\rightarrow} A_{0} \cup A_{0}^{*}$, e daí seguem-se três casos a considerar:

Caso 1: $S(t) x \underset{t \rightarrow \infty}{\rightarrow} A_{0} \operatorname{com} l_{0}(x)>0$.

Neste caso, escolhamos $0<\theta<l_{0}(x)$. Uma vez que $l_{0}: X \rightarrow \mathbb{R}$ é contínua (confira a prova da Proposição 4.2.2), existe $\sigma_{1}>0$ tal que $l_{0}\left(\mathcal{O}_{\sigma_{1}}(x)\right) \subset\left(\frac{\theta}{2}, 1\right]$. Usando a convergência $l_{\eta} \underset{\eta \rightarrow 0_{+}}{\rightarrow} l_{0}$ obtida no Passo 1 , existe $\eta_{0} \in(0,1]$ tal que $l_{\eta}\left(t, \mathcal{O}_{\sigma_{1}}(x)\right) \subset(\theta, 1]$ para $\eta \in\left[0, \eta_{0}\right]$ e $t \in \mathbb{R}$ quaisquer. 
Usando, novamente, a continuidade da função $l_{0}: X \rightarrow \mathbb{R}$, dado $0<\alpha<\frac{\theta}{2}$, existe $\delta>0$ de maneira que $l_{0}\left(\mathcal{O}_{\delta}\left(A_{0}\right)\right) \subset[0, \alpha)$. Agora, devido ao Lema 7.5.9, existem $\delta^{\prime} \in\left(0, \frac{\delta}{2}\right)$ e $\eta_{1} \in\left(0, \eta_{0}\right]$ tais que

$$
T_{\eta}(t, s)\left(\mathcal{O}_{\delta^{\prime}}\left(A_{\eta}(s)\right)\right) \subset \mathcal{O}_{\frac{\delta}{2}}\left(A_{\eta}(t)\right) \text { para } t \geq s \text { e } \eta \in\left[0, \eta_{1}\right] \text { quaisquer. }
$$

Pela semicontinuidade inferior de $\left(A_{\eta}\right)_{\eta \in[0,1]}$ em $\eta=0$, podemos escolher $\eta_{2} \in\left(0, \eta_{1}\right]$ tal que

$$
A_{0} \subset \mathcal{O}_{\frac{\delta^{\prime}}{2}}\left(A_{\eta}(t)\right) \text { para todo } t \in \mathbb{R} \text { e } \eta \in\left[0, \eta_{2}\right] .
$$

Pelo fato de que $S(t) x \underset{t \rightarrow \infty}{\rightarrow} A_{0}$, escolhamos $t_{0}>0$ de maneira que $S\left(t_{0}\right) x \in \mathcal{O}_{\frac{\delta^{\prime}}{4}}\left(A_{0}\right) \mathrm{e}$ pela continuidade da aplicação $S\left(t_{0}\right): X \rightarrow X$ seja $\sigma_{2} \in\left(0, \sigma_{1}\right]$ tal que $S\left(t_{0}\right)\left(\mathcal{O}_{\sigma_{2}}(x)\right) \subset$ $\mathcal{O}_{\frac{\delta^{\prime}}{4}}\left(A_{0}\right)$. Graças a hipótese de convergência dada em $(d)$, podemos encontrar $\sigma_{3} \in$ $\left(0, \sigma_{2}\right]$ e $\eta_{3} \in\left(0, \eta_{2}\right]$ tais que para todo $\eta \in\left[0, \eta_{3}\right]$ tem-se $T_{\eta}\left(t_{0}+s, s\right)\left(\mathcal{O}_{\sigma_{3}}(x)\right) \subset \mathcal{O}_{\frac{\delta^{\prime}}{2}}\left(A_{0}\right)$ qualquer que seja $s \in \mathbb{R}$. Daí e de (7.5.46), obtém-se $T_{\eta}\left(t_{0}+s, s\right)\left(\mathcal{O}_{\sigma_{3}}(x)\right) \subset \mathcal{O}_{\delta^{\prime}}\left(A_{\eta}(t)\right)$ para todo par $s$ e $t$ de números reais, sempre que $\eta \in\left[0, \eta_{3}\right]$, e de (7.5.45), em particular, conclui-se que

$$
T_{\eta}(t+s, s)\left(\mathcal{O}_{\sigma_{3}}(x)\right) \subset \mathcal{O}_{\frac{\delta}{2}}\left(A_{\eta}(t+s)\right) \text { para todo } t \geq t_{0}, \eta \in\left[0, \eta_{3}\right] \text { e } s \in \mathbb{R} \text {. }
$$

Por outro lado, observemos que, da convergência uniforme $l_{\eta} \underset{\eta \rightarrow 0^{+}}{\rightarrow} l_{0}$ em $\mathbb{R} \times X$, obtém-se $\eta_{4} \in\left(0, \eta_{3}\right]$ de maneira que, para todo $\eta \in\left[0, \eta_{4}\right], l_{\eta}\left(t, \mathcal{O}_{\delta}\left(A_{0}\right)\right) \subset[0,2 \alpha)$ para todo $t \in \mathbb{R}$. Pela semicontinuidade superior da família $\left(A_{\eta}\right)_{\eta \in[0,1]}$ em $\eta=0$ existe $\eta_{5} \in\left(0, \eta_{4}\right]$ de modo que, se $\eta \in\left[0, \eta_{5}\right]$, então $A_{\eta}(t) \subset \mathcal{O}_{\frac{\delta}{2}}\left(A_{0}\right)$ para todo $t \in \mathbb{R}$. Daí, $\mathcal{O}_{\frac{\delta}{2}}\left(A_{\eta}(t)\right) \subset \mathcal{O}_{\delta}\left(A_{0}\right)$ para todo $\eta \in\left[0, \eta_{5}\right]$ e $t \in \mathbb{R}$. Então, $l_{\eta}\left(t, \mathcal{O}_{\frac{\delta}{2}}\left(A_{\eta}(\tau)\right)\right) \subset[0,2 \alpha)$ para todo $\eta \in\left[0, \eta_{5}\right]$ e $t$ e $\tau$ em $\mathbb{R}$. De (7.5.47) conclui-se que

$$
\sup _{t \geq t_{0}} l_{\eta}\left(t+s, T_{\eta}(t+s, s) z\right) \leq 2 \alpha<\theta<l_{\eta}(s, z) \leq k_{\eta}(s, z),
$$

para cada $\eta \in\left[0, \eta_{5}\right], s \in \mathbb{R}$ e $z \in \mathcal{O}_{\sigma_{3}}(x) \subset \mathcal{O}_{\sigma_{1}}(x)$. Consequentemente

$$
k_{\eta}(s, z)=\sup _{0 \leq t \leq t_{0}} l_{\eta}\left(t+s, T_{\eta}(t+s, s) z\right)
$$

para todo $\eta \in\left[0, \eta_{5}\right], s \in \mathbb{R}$ e $z \in \mathcal{O}_{\sigma_{3}}(x)$.

Finalmente, dado $\varepsilon>0$, pelo Paso 1 , existe $\eta_{6} \in\left(0, \eta_{5}\right]$ tal que

$$
\left|l_{\eta}(t, w)-l_{0}(w)\right|<\frac{\varepsilon}{2} \text { para todo } \eta \in\left[0, \eta_{6}\right], w \in X \text { e } t \in \mathbb{R} .
$$

Pela continuidade uniforme da função $l_{0}: X \rightarrow \mathbb{R}$, consideremos $\beta>0$ tal que se $w$ e $w^{\prime}$ estão $X$ e satisfazem $d\left(w, w^{\prime}\right)<\beta$ então $\left|l_{0}(w)-l_{0}\left(w^{\prime}\right)\right|<\frac{\varepsilon}{2}$ e, pela hipótese de convergência $(d)$ podemos escolher $\eta_{7} \in\left(0, \eta_{6}\right]$ e $\sigma_{4} \in\left(0, \sigma_{3}\right]$ tais que

$$
\sup _{s \in \mathbb{R}} \sup _{z \in \mathcal{O}_{\sigma_{4}}(x)} \sup _{0 \leq t \leq t_{0}} d\left(T_{\eta}(t+s, s) z, S(t) z\right)<\beta \text { para todo } \eta \in\left[0, \eta_{7}\right] .
$$


Daí, podemos escrever

$$
\begin{gathered}
\left|l_{\eta}\left(t+s, T_{\eta}(t+s, s) z\right)-l_{0}(S(t) z)\right| \leq \\
\left|l_{\eta}\left(t+s, T_{\eta}(t+s, s) z\right)-l_{0}\left(T_{\eta}(t+s, s) z\right)\right|+\left|l_{0}\left(T_{\eta}(t+s, s) z\right)-l_{0}\left(T_{0}(t) z\right)\right|<\varepsilon,
\end{gathered}
$$

para todo $z \in \mathcal{O}_{\sigma_{4}}(x), t \in\left[0, t_{0}\right], s \in \mathbb{R}$ e $\eta \in\left[0, \eta_{7}\right]$. O que implica

$$
\sup _{s \in \mathbb{R}} \sup _{z \in \mathcal{O}_{\sigma_{4}}(x)}\left|k_{\eta}(s, z)-k_{0}(z)\right| \leq \varepsilon \text { sempre que } \eta \in\left[0, \eta_{7}\right] \text {, }
$$

onde $\sigma_{4}>0$ e $\eta_{7}>0$ dependem somente do ponto $x \in X$, do conjunto $A_{0}$ e do $\varepsilon>0$ dado.

Caso 2: $l_{0}(x)=0$.

Neste caso temos $x \in A_{0}$ e, consequentemente, $k_{0}(x)=0$ (porque $S(t) x \in A_{0}$ para todo $t \geq 0$ e $\left.l_{0}^{-1}(0)=A_{0}\right)$.

Dado $\varepsilon>0$, pela continuidade de $l_{0}: X \rightarrow \mathbb{R}$, tomemos $\delta>0$ tal que $l_{0}\left(\mathcal{O}_{\delta}\left(A_{0}\right)\right) \subset$ $\left[0, \frac{\varepsilon}{4}\right)$.

Agora, devido a convergência uniforme de $\left(l_{\eta}\right)_{\eta \in[0,1]}$ para $l_{0}$ em todo o produto $\mathbb{R} \times X$ existe $\eta_{0} \in(0,1]$ tal que

$$
l_{\eta}\left(t, \mathcal{O}_{\delta}\left(A_{0}\right)\right) \subset\left[0, \frac{\varepsilon}{2}\right) \text { para cada } \eta \in\left[0, \eta_{0}\right] \text { e } t \in \mathbb{R} .
$$

Da semicontinuidade superior de $\left(A_{\eta}\right)_{\eta \in[0,1]}$ em $\eta=0$ obtém-se a existência de $\eta_{1} \in\left(0, \eta_{0}\right]$ tal que para todo $\eta \in\left[0, \eta_{1}\right]$ e todo $t \in \mathbb{R}$ tem-se $A_{\eta}(t) \subset \mathcal{O}_{\frac{\delta}{2}}\left(A_{0}\right)$, donde conclui-se que $\mathcal{O}_{\frac{\delta}{2}}\left(A_{\eta}(t)\right) \subset \mathcal{O}_{\delta}\left(A_{0}\right)$ se $\eta \in\left[0, \eta_{1}\right]$ e $t \in \mathbb{R}$. De (7.5.49) resulta que para todo $\eta \in\left[0, \eta_{1}\right]$

$$
l_{\eta}\left(t, \mathcal{O}_{\frac{\delta}{2}}\left(A_{\eta}(s)\right)\right) \subset\left[0, \frac{\varepsilon}{2}\right) \text { quaisquer que sejam } s \text { e } t \text { em } \mathbb{R} .
$$

Escolhamos agora $\eta_{2} \in\left(0, \eta_{1}\right]$ e $\delta^{\prime} \in\left(0, \frac{\delta}{2}\right)$, graças ao Lema 7.5.9, tais que

$$
T_{\eta}(t, s)\left(\mathcal{O}_{\delta^{\prime}}\left(A_{\eta}(s)\right)\right) \subset \mathcal{O}_{\frac{\delta}{2}}\left(A_{\eta}(t)\right) \text { toda vez que } t \geq s \text { e } \eta \in\left[0, \eta_{2}\right] .
$$

Finalmente, pela semicontinuidade inferior da família $\left(A_{\eta}\right)_{\eta \in[0,1]}$ em $\eta=0$ existe $\eta_{3} \in\left(0, \eta_{2}\right]$ tal que

$$
A_{0} \subset \mathcal{O}_{\frac{\delta^{\prime}}{2}}\left(A_{\eta}(t)\right) \text { para todo } \eta \in\left[0, \eta_{3}\right] \text { e } t \in \mathbb{R} .
$$

Assim, por (7.5.52) e por (7.5.51), para $\eta \in\left[0, \eta_{3}\right], s \in \mathbb{R}$ e $z \in \mathcal{O}_{\frac{\delta^{\prime}}{2}}\left(A_{0}\right) \subset \mathcal{O}_{\delta^{\prime}}\left(A_{\eta}(s)\right)$, obtém-se que $T_{\eta}(t+s, s) z \in \mathcal{O}_{\frac{\delta}{2}}\left(A_{\eta}(t+s)\right)$ para todo $t \geq 0$, e então, de (7.5.50), segue-se que

$$
k_{\eta}(s, z)=\sup _{t \geq 0} l_{\eta}\left(t+s, T_{\eta}(t+s, s) z\right) \leq \frac{\varepsilon}{2},
$$


para todo $\eta \in\left[0, \eta_{3}\right], s \in \mathbb{R}$ e $z \in \mathcal{O}_{\frac{\delta^{\prime}}{2}}\left(A_{0}\right)$. Em particular,

$$
\sup _{s \in \mathbb{R}} \sup _{z \in \mathcal{O}_{\frac{\delta^{\prime}}{2}}\left(A_{0}\right)}\left|k_{\eta}(s, z)-k_{0}(z)\right| \leq \varepsilon \text { para todo } \eta \in\left[0, \eta_{3}\right]
$$

onde $\delta^{\prime}>0$ e $\eta_{3}>0$ dependem somente de $A_{0}$ e do $\varepsilon>0$ dado.

Caso 3: $S(t) x \underset{t \rightarrow \infty}{\rightarrow} A_{0}^{*}$.

Neste caso é fácil ver que $k_{0}(x)=1$. Pela continuidade de $l_{0}: X \rightarrow \mathbb{R}$, dado $\varepsilon>0$, seja $\delta>0$ tal que

$$
l_{0}\left(\mathcal{O}_{\delta}\left(A_{0}^{*}\right)\right) \subset\left(1-\frac{\varepsilon}{4}, 1\right]
$$

e, pela convergência uniforme $l_{\eta} \underset{\eta \rightarrow 0^{+}}{\rightarrow} l_{0}$ em $\mathbb{R} \times X$, seja $\eta_{0} \in(0,1]$ tal que

$$
l_{\eta}\left(t, \mathcal{O}_{\delta}\left(A_{0}^{*}\right)\right) \subset\left(1-\frac{\varepsilon}{2}, 1\right] \text { para } \eta \in\left[0, \eta_{0}\right] \text { e } t \in \mathbb{R} .
$$

Por outro lado, consideremos $t_{0}>0$ tal que $S\left(t_{0}\right) x \in \mathcal{O}_{\frac{\delta}{2}}\left(A_{0}^{*}\right)$ e, pela continuidade da aplicação $S\left(t_{0}\right): X \rightarrow X$, tomemos $\sigma_{1}>0$ tal que $S\left(t_{0}\right)\left(\mathcal{O}_{\sigma_{1}}(x)\right) \subset \mathcal{O}_{\frac{\delta}{2}}\left(A_{0}^{*}\right)$. Usando a hipótese de convergência $(d)$, sejam $\eta_{1} \in\left(0, \eta_{0}\right]$ e $\sigma_{2} \in\left(0, \sigma_{1}\right]$ tais que $T_{\eta}\left(t_{0}+\right.$ $s, s)\left(\mathcal{O}_{\sigma_{2}}(x)\right) \subset \mathcal{O}_{\delta}\left(A_{0}^{*}\right)$ para $\eta \in\left[0, \eta_{1}\right]$ e $s \in \mathbb{R}$ quaisquer.

Finalmente, de (7.5.54) deduz-se que $l_{\eta}\left(t_{0}+s, T_{\eta}\left(t_{0}+s, s\right)\left(\mathcal{O}_{\sigma_{2}}(x)\right)\right) \subset\left(1-\frac{\varepsilon}{2}, 1\right]$ para todo $\eta \in\left[0, \eta_{1}\right]$ e todo $s \in \mathbb{R}$. Portanto, $1-\frac{\varepsilon}{2}<l_{\eta}\left(t_{0}+s, T_{\eta}\left(t_{0}+s, s\right) z\right) \leq$ $k_{\eta}(s, z) \leq 1$ quaisquer que sejam $z \in \mathcal{O}_{\sigma_{2}}(x), s \in \mathbb{R}$ e $\eta \in\left[0, \eta_{1}\right]$. Isto obriga a que $\left|k_{\eta}(s, z)-k_{0}(z)\right| \leq \varepsilon$ para $\eta \in\left[0, \eta_{1}\right], s \in \mathbb{R}$ e $z \in \mathcal{O}_{\sigma_{2}}(x)$ e então

$$
\sup _{s \in \mathbb{R}} \sup _{z \in \mathcal{O}_{\sigma_{2}}(x)}\left|k_{\eta}(s, z)-k_{0}(z)\right| \leq \varepsilon \text { sempre que } \eta \in\left[0, \eta_{1}\right]
$$

onde $\sigma_{2}>0$ e $\eta_{1}$ dependem apenas do conjunto $A_{0}^{*}$, do ponto $x \in X$ e do $\varepsilon>0$ dado.

Agora, reunindo as conlusões dos Casos 1, 2 e 3 obtém-se que:

Dados um subconjunto compacto $K \subset X$ e $\varepsilon>0$, por (7.5.48), (7.5.53) e (7.5.55), existe um conjunto aberto $U=U(\varepsilon, K) \subset X$, com $K \subset U$, e um índice $\eta^{\prime}=\eta^{\prime}(\varepsilon, K)>$ 0 tais que

$$
\sup _{s \in \mathbb{R}} \sup _{x \in U}\left|k_{\eta}(s, x)-k_{0}(x)\right| \leq \varepsilon \text { sempre que } \eta \in\left[0, \eta^{\prime}\right],
$$

donde conclui-se que $\lim _{\eta \rightarrow 0^{+}} \operatorname{supsup}_{s \in \mathbb{R} x \in K}\left|k_{\eta}(s, x)-k_{0}(x)\right|=0$.

Passo 3: Para todo conjunto compacto $K \subset X$ temos

$$
\lim _{\eta \rightarrow 0^{+}} \sup _{t \in \mathbb{R}} \sup _{x \in K}\left|h_{\eta}(t, x)-h_{0}(x)\right|=0 .
$$

De fato, dado $x \in X$ consideraremos agora dois casos:

Caso 1: $d\left(x, \mathcal{A}_{0}\right)>0$. 
Assumindo esta condição, dado $\alpha>0$ com $0<\alpha<d\left(x, \mathcal{A}_{0}\right)$, sejam, pelo Lema 7.5.9, $\alpha^{\prime} \in(0, \alpha)$ e $\eta_{0} \in(0,1]$ tais que

$$
T_{\eta}(t, s)\left(\mathcal{O}_{\alpha^{\prime}}\left(\mathcal{A}_{\eta}(s)\right)\right) \subset \mathcal{O}_{\alpha}\left(\mathcal{A}_{\eta}(t)\right) \text { sempre que } t \geq s \text { e } \eta \in\left[0, \eta_{0}\right]
$$

Escolhamos $t_{0}>0$ tal que $S\left(t_{0}\right) x \in \mathcal{O}_{\frac{\alpha^{\prime}}{4}}\left(\mathcal{A}_{0}\right)$ e, pela continuidade da aplicação $S\left(t_{0}\right): X \rightarrow X$, seja $\sigma_{1}>0$ tal que $S\left(t_{0}\right)\left(\mathcal{O}_{\sigma_{1}}(x)\right) \subset \mathcal{O}_{\frac{\alpha^{\prime}}{4}}\left(\mathcal{A}_{0}\right)$.

Agora, pela hipótese de convergência $(d)$, sejam $\eta_{1} \in\left(0, \eta_{0}\right]$ e $\sigma_{2} \in\left(0, \sigma_{1}\right]$ tais que $T_{\eta}\left(t_{0}+s, s\right)\left(\mathcal{O}_{\sigma_{2}}(x)\right) \subset \mathcal{O}_{\frac{\alpha^{\prime}}{2}}\left(\mathcal{A}_{0}\right)$ para todo $\eta \in\left[0, \eta_{1}\right]$ e todo $s \in \mathbb{R}$, e, pela semicontinuidade inferior de $\left(\mathcal{A}_{\eta}\right)_{\eta \in[0,1]}$ em $\eta=0$, escolhamos $\eta_{2} \in\left(0, \eta_{1}\right]$ tal que $\mathcal{A}_{0} \subset \mathcal{O}_{\frac{\alpha^{\prime}}{2}}\left(\mathcal{A}_{\eta}(t)\right)$ para todo $\eta \in\left[0, \eta_{2}\right]$ e $t \in \mathbb{R}$. Portanto, $\mathcal{O}_{\frac{\alpha^{\prime}}{2}}\left(\mathcal{A}_{0}\right) \subset \mathcal{O}_{\alpha^{\prime}}\left(\mathcal{A}_{\eta}(t)\right)$ para $\eta \in\left[0, \eta_{2}\right]$ e $t \in \mathbb{R}$ quaisquer. Em particular, $T_{\eta}\left(t_{0}+s, s\right)\left(\mathcal{O}_{\sigma_{2}}(x)\right) \subset \mathcal{O}_{\alpha^{\prime}}\left(\mathcal{A}_{\eta}\left(t_{0}+s\right)\right)$ para todo $\eta \in\left[0, \eta_{2}\right]$ e $s \in \mathbb{R}$. De (7.5.56) obtém-se $T_{\eta}(t+s, s)\left(\mathcal{O}_{\sigma_{2}}(x)\right) \subset \mathcal{O}_{\alpha}\left(\mathcal{A}_{\eta}(t+s)\right)$ para todo $\eta \in\left[0, \eta_{2}\right], s \in \mathbb{R}$ e $t \geq t_{0}$. Consequentemente

$$
\sup _{t \geq t_{0}} d\left(T_{\eta}(t+s, s) z, \mathcal{A}_{\eta}(t+s)\right) \leq \alpha \text { para } \eta \in\left[0, \eta_{2}\right], s \in \mathbb{R} \text { e } z \in \mathcal{O}_{\sigma_{2}}(x) .
$$

Por outro lado, pela desigualdade triangular da semidistância de Hausdorff, quaisquer que sejam o ponto $z \in X, t \in \mathbb{R}$ e $\eta \in[0,1]$ tem-se

$$
\left|d\left(z, \mathcal{A}_{\eta}(t)\right)-d\left(z, \mathcal{A}_{0}\right)\right| \leq d_{H}\left(\mathcal{A}_{\eta}(t), \mathcal{A}_{0}\right)
$$

Então, se escolhemos $\eta_{3} \in\left(0, \eta_{2}\right]$ e $\sigma_{3} \in\left(0, \sigma_{2}\right]$ tais que $d\left(z, \mathcal{A}_{\eta}(t)\right)>\alpha$ para todo $\eta \in\left[0, \eta_{3}\right], t \in \mathbb{R}$ e $z \in \mathcal{O}_{\sigma_{3}}(x)$ concluímos, de (7.5.57), que

$$
\sup _{t \geq t_{0}} d\left(T_{\eta}(t+s, s) z, \mathcal{A}_{\eta}(t+s)\right) \leq \alpha<d\left(z, \mathcal{A}_{\eta}(s)\right)
$$

sempre que $\eta \in\left[0, \eta_{3}\right], s \in \mathbb{R}$ e $z \in \mathcal{O}_{\sigma_{3}}(x)$. Então, $h_{\eta}(s, z)=\sup _{0 \leq t \leq t_{0}} d\left(T_{\eta}(t+s, s) z, \mathcal{A}_{\eta}(t+\right.$ $s))$ para $\eta \in\left[0, \eta_{3}\right], s \in \mathbb{R}$ e $z \in \mathcal{O}_{\sigma_{3}}(x)$.

Observemos agora que, também pela desigualdade triangular da semidistância de Hausdorff, para todo $z \in X, \eta \in[0,1], s \in \mathbb{R}$ e $t \geq 0$ temos

$$
\left|d\left(T_{\eta}(t+s, s) z, \mathcal{A}_{\eta}(t+s)\right)-d\left(S(t) z, \mathcal{A}_{0}\right)\right| \leq d_{H}\left(\mathcal{A}_{\eta}(t+s), \mathcal{A}_{0}\right)+d\left(T_{\eta}(t+s, s) z, S(t) z\right),
$$

donde, para todo $\eta \in\left[0, \eta_{3}\right]$

$\sup _{s \in \mathbb{R}} \sup _{z \in \mathcal{O}_{\sigma_{3}}(x)}\left|h_{\eta}(s, z)-h_{0}(z)\right| \leq \sup _{s \in \mathbb{R}} d_{H}\left(\mathcal{A}_{\eta}(s), \mathcal{A}_{0}\right)+\sup _{s \in \mathbb{R}} \sup _{z \in \mathcal{O}_{\sigma_{3}}(x)} \sup _{0 \leq t \leq t_{0}} d\left(T_{\eta}(t+s, s) z, S(t) z\right)$,

e daí conclui-se facilmente que, dado $\varepsilon>0$ existem $\sigma_{4} \in\left(0, \sigma_{3}\right]$ e $\eta_{4} \in\left(0, \eta_{3}\right]$ tais que

$$
\sup _{s \in \mathbb{R}} \sup _{z \in \mathcal{O}_{\sigma_{4}}(x)}\left|h_{\eta}(s, z)-h_{0}(z)\right| \leq \varepsilon \text { sempre que } \eta \in\left[0, \eta_{4}\right]
$$


Caso 2: $d\left(x, \mathcal{A}_{0}\right)=0$, isto é, $x \in \mathcal{A}_{0}$.

Neste caso, pelo Lema 7.5.9, dado $\varepsilon>0$, existem $\varepsilon^{\prime} \in\left(0, \frac{\varepsilon}{2}\right)$ e $\eta_{0} \in(0,1]$ tais que

$$
T_{\eta}(t, s)\left(\mathcal{O}_{\varepsilon^{\prime}}\left(\mathcal{A}_{\eta}(s)\right)\right) \subset \mathcal{O}_{\frac{\varepsilon}{2}}\left(\mathcal{A}_{\eta}(t)\right) \text { para } t \geq s \text { e } \eta \in\left[0, \eta_{0}\right]
$$

Também, como $\left(\mathcal{A}_{\eta}\right)_{\eta \in[0,1]}$ é semicontínua inferiormente em $\eta=0$, podemos escolher $\eta_{1} \in\left(0, \eta_{0}\right]$ tal que $\mathcal{A}_{0} \subset \mathcal{O}_{\frac{\varepsilon^{\prime}}{2}}\left(\mathcal{A}_{\eta}(s)\right)$ toda vez que $\eta \in\left[0, \eta_{1}\right]$ e $s \in \mathbb{R}$ é qualquer. Daí segue-se então que $\mathcal{O}_{\frac{\varepsilon^{\prime}}{2}}\left(\mathcal{A}_{0}\right) \stackrel{\mathcal{C}^{\prime}}{\subset} \mathcal{O}_{\varepsilon^{\prime}}\left(\mathcal{A}_{\eta}(s)\right)$ para todo $\eta \in\left[0, \eta_{1}\right]$ e $s \in \mathbb{R}$. Por este último fato e por (7.5.59) temos

$$
T_{\eta}(t, s)\left(\mathcal{O}_{\frac{\varepsilon^{\prime}}{2}}\left(\mathcal{A}_{0}\right)\right) \subset \mathcal{O}_{\frac{\varepsilon}{2}}\left(\mathcal{A}_{\eta}(t)\right) \text { se } \eta \in\left[0, \eta_{1}\right] \text { e } t \geq s
$$

Consequentemente, $h_{\eta}(s, z)=\sup _{t \geq 0} d\left(T_{\eta}(t+s, s) z, \mathcal{A}_{\eta}(t+s)\right) \leq \frac{\varepsilon}{2}$ sempre que $\eta \in\left[0, \eta_{1}\right]$ e $z \in \mathcal{O}_{\frac{\varepsilon^{\prime}}{2}}\left(\mathcal{A}_{0}\right)$, donde conclui-se que

$$
\sup _{s \in \mathbb{R}} \sup _{z \in \mathcal{O}_{\frac{\varepsilon^{\prime}}{2}}\left(\mathcal{A}_{0}\right)}\left|h_{\eta}(s, z)-h_{0}(z)\right| \leq \varepsilon \text { para todo } \eta \in\left[0, \eta_{1}\right]
$$

Das conclusões obtidas nos Casos 1 e 2 resulta que, dados $\varepsilon>0$ e $x \in X$ existem $\sigma=\sigma(\varepsilon, x)>0$ e $\eta^{\prime}=\eta^{\prime}(\varepsilon, z)>0$ tais que

$$
\sup _{s \in \mathbb{R}} \sup _{w \in \mathcal{O}_{\sigma}(x)}\left|h_{\eta}(s, z)-h_{0}(z)\right| \leq \varepsilon \text { toda vez que } \eta \in\left[0, \eta^{\prime}\right] .
$$

Assim, dado um compacto $K \subset X$, analogamente ao que fizemos no final do Paso 2, podemos concluir a convergência $\lim _{\eta \rightarrow 0^{+}} \operatorname{supsup}_{t \in \mathbb{R}}\left|h_{\eta \in K}(t, x)-h_{0}(x)\right|=0$, e este fato completa a demonstração. 



\section{Aplicação a problemas com acoplamento unilateral}

Neste capítulo, apresentamos um exemplo concreto onde podemos aplicar os resultados abstratos discutidos nos capítulos anteriores, mais precisamente, usaremos o Teorema 4.4.1 para demonstrar que um certo tipo de semigrupo, associado a uma equação diferencial, é gradiente. Em outras palavras, provreamos que o mesmo é de tipo gradiente e, portanto, gradiente.

Diferentemente do que ocorreu nos capítulos anteriores, este, por sua vez, não é inteiramente autocontido. Utilizamos aqui, a fim de estabelecer, de forma precisa, o teorema principal deste capítulo, a nomenclatura básica da teoria dos semigrupos lineares, que está aplamente difundida na literatura (Confira, por exemplo, [14] e [22]) e, implicitamente, os resultados clássicos sobre existência local e global de soluções para problemas semilineares associados.

Comecemos com um pouco mais de teoria abstrata, onde fixamos o vacabulário usado e apresentamos o problema. Em seguida, introduzimos uma classe de equações, que serão estudadas no contexto dos espaços de Hilbert, onde os resultados aqui desenvolvidos podem ser aplicados. Além disso, apresentamos a Desigualdade de Lojasiewicz-Simon, que é de fundamental importância no estudo de sistemas dinâmicos autônomos, possuindo conjunto infinito de pontos de equilíbrios e evoluindo em espaços de Hilbert. 


\subsection{Teoria abstrata}

A fim de permitir uma maior aplicabilidade dos resultados aqui obtidos, mudaremos ligeiramente a definição de semigrupos de tipo gradiente para que eles, agora, admitam conjuntos de equilíbrios não necessariamente finitos. Recordemos, contudo, que a hipótese de finitude do conjunto de equilíbrios (ou mais geralmente, do conjunto de invariantes isolados) foi essencial para se estabelecer todos os resultados apresentados nos capítulos anteriores, desta forma, seremos levados a impor novas condições para realizarmos esta tarefa, que a seguir iniciamos.

Primeiramente, apresentemos a nova definição de semigrupos de tipo gradiente.

Definição 8.1.1. Sejam $X$ um espaço métrico com distância $d: X \times X \rightarrow \mathbb{R} e$ $\{T(t): t \geq 0\}$ um semigrupo em $X$ possuindo atrator global $\mathcal{A}$ e conjunto de pontos estacionários (possivelmente infinito) $\mathcal{E}$. Diz-se que $\{T(t): t \geq 0\}$ é um semigrupo de tipo gradiente com respeito a $\mathcal{E}$ quando as duas seguintes propridades estão satisfeitas:

$\left(G 1^{\prime}\right)$ Se $\xi: \mathbb{R} \rightarrow X$ é uma solução global limitada de $\{T(t): t \geq 0\}$, existem pontos de equilíbrios $z^{*}$ e $w^{*}$ em $\mathcal{E}$ tais que

$$
\lim _{t \rightarrow-\infty} d\left(\xi(t), z^{*}\right)=0 \text { e } \lim _{t \rightarrow \infty} d\left(\xi(t), w^{*}\right)=0 .
$$

$\left(G 2^{\prime}\right) O$ atrator $\mathcal{A}$ de $\{T(t): t \geq 0\}$ não contém estruturas homoclínicas (relativas a $\mathcal{E})$, no sentido da Definição 2.2.1.

A seguir descrevemos alguns fatos, de ordem teórica, necessários para que possamos tratar um problema semilinear autônomo abstrato com acoplamento parcial (ou unilateral) do tipo

$$
\left\{\begin{array}{l}
\dot{x}=A x+g(x) t>0 \\
\dot{y}=B y+f(x, y) t>0 \\
x(0)=x_{0} \in X, y(0)=y_{0} \in Y
\end{array} .\right.
$$

Onde $X$ e $Y$ são espaços de Banach, $A: D(A) \subset X \rightarrow X$ e $B: D(B) \subset Y \rightarrow$ $Y$ geradores de $C_{0}$-semigrupos, $f: X \times Y \rightarrow Y$ uma aplicação possuindo derivada parcial de primeira ordem com relação à segunda variável contínua e $g: X \rightarrow X$ uma aplicação continuamente diferenciável. Seja $D\left(A_{0}\right)$ um subespaço vetorial de $D(A) \times D(B)$ (possivelmente todo $D(A) \times D(B)$ ) denso em $Z:=X \times Y$ (onde $Z$ está, naturalmente, dotado da norma produto). Consideremos o operador linear diagonal $A_{0}: D\left(A_{0}\right) \subset Z \rightarrow Z$ definido por $A_{0} z:=A_{0}(x, y):=(A x, B y)$ para $z=(x, y) \in D\left(A_{0}\right)$ e suponhamos que $A_{0}$ seja gerador de um $C_{0}$-semigrupo em $Z$ (ou 
ainda, gerador de um semigrupo singular. Confira [5] para um exemplo concreto desta situação singular). Definamos $h: Z \rightarrow Z$ pondo $h(z):=h(x, y):=(g(x), f(x, y))$ para $z=(x, y) \in Z$. Nestas condições, o problema (8.1.1) pode escrever-se da seguinte maneira

$$
\left\{\begin{array}{l}
\dot{z}=A_{0} z+h(z) t>0 \\
z(0)=z_{0} \in Z
\end{array}\right.
$$

Suponhamos também, que as aplicações $f: X \times Y \rightarrow Y$ e $g: X \rightarrow X$ sejam tais que os problemas (8.1.2) e

$$
\left\{\begin{array}{l}
\dot{x}=A x+g(x) t>0 \\
x(0)=x_{0} \in X
\end{array}\right.
$$

sejam geradores, respectivamente, de um semigrupo $\{T(t): t \geq 0\}$ em $Z$ possuindo atrator global $\mathcal{A}$ e conjunto de pontos de equilíbrio $\mathcal{E}$ e um semigrupo de tipo gradiente $\{S(t): t \geq 0\}$ em $X$, com respeito ao conjunto de pontos de equilíbrios $\mathcal{S}$ e possuindo atrator global $\mathcal{A}_{0}$.

Aqui, por "gerar semigrupo" estamos entendendo que o operador solução da equação define um semigrupo.

De agora em diante, sempre que dissermos que um determinado sistema de equações é (gradiente ou) de tipo gradiente, queremos dizer que este sistema possui como operador solução um semigrupo (gradiente ou) de tipo gradiente.

O objetivo deste capítulo é, levando em consideração o contexto acima, estudar condições para que o semigrupo $\{T(t): t \geq 0\}$ associado a (8.1.2) seja um semigrupo de tipo gradiente e, no caso particular onde seu conjunto de pontos estacionários é finito, usar o Teorema 4.4.1 para concluir que o referido semigrupo é um semigrupo gradiente.

As duas definições seguintes são necessárias para que possamos enunciar o próximo resultado, o qual foi extraído de [11], e que utilizamos na prova do principal teorema deste capítulo. Estes dois novos conceitos, que costumam ser chamados de condições de hiperbolicidade, são, precisamente, as situações mais comuns onde encontramos satisfeitas as condições de invariância isolada, como apresentamos nas Definições 2.1.1 e 7.2.1.

Definição 8.1.2. Um ponto de equilíbrio $x_{0}^{*}$ para o problema

$$
\left\{\begin{array}{l}
\dot{x}=L x+F(x), t>0 \\
x(0)=x_{0} \in X
\end{array},\right.
$$

onde $L: D(L) \subset X \rightarrow X$ é um gerador de $C_{0}$-semigrupo num espaço de Banach $X$ e $F: X \rightarrow X$ é uma aplicação continuamente (Fréchet) diferenciável, chama-se hiperbólico, quando verificam-se as condições abaixo: 
(i) O espectro do operador linear $D:=L+F^{\prime}\left(x_{0}^{*}\right): D(L) \subset X \rightarrow X$ não intersepta o eixo imaginário do plano complexo $\mathbb{C}$.

(ii) O conjunto $\sigma^{+}:=\{\lambda \in \sigma(D): \operatorname{Re} \lambda>0\}$ é compacto $e$,

(iii) Se $\gamma$ é uma curva suave fechada com traço em $\rho(D) \cap\{\lambda \in \mathbb{C}: \operatorname{Re} \lambda>0\}$, orientada em sentido anti-horário, contendo $\sigma^{+}$em seu interior geométrico, pondo

$$
P:=P\left(\sigma^{+}\right):=\frac{1}{2 \pi i} \int_{\gamma}(\lambda-D)^{-1} d \lambda
$$

então, para $t \geq 0, P e^{D t}=e^{D t} P$ é um isomorfismo de $R(P)$ sobre si mesmo, com inverso indicado por $e^{-t D} P, R(P) \subset D(D)$ e existem constantes $K \geqslant 1$ e $\beta>0$ tais que

$$
\begin{aligned}
& \left\|e^{D t} P\right\|_{\mathcal{L}(X)} \leqslant K e^{\beta t}, \text { para todo } t \leqslant 0, \\
& \left\|e^{D t}(I-P)\right\|_{\mathcal{L}(X)} \leqslant K e^{-\beta t}, \text { para todo } t \geqslant 0 .
\end{aligned}
$$

A próxima definição, que diz respeito à noção de dicotomia exponencial para um processo de evolução linear e a de solução global hiperbólica, é o análogo não autônomo da definição anterior.

Definição 8.1.3. Diz-se que um processo de evolução linear $\{U(t, s): t \geq s\} \subset$ $\mathcal{L}(X)$ em um espaço de Banach $X$ possui dicotomia exponencial com expoente $\beta>0$ e constante $K$, quando existe uma família de projeções $\{P(t): t \in \mathbb{R}\} \subset \mathcal{L}(X)$ satisfazendo as três condições seguintes:

(i) $P(t) U(t, s)=U(t, s) P(s)$, para todos os reais $t$ e $s$ com $t \geqslant s$;

(ii) Se $t \geq s$ a restrição $\left.U(t, s)\right|_{R(P(s))}$ é um isomorfismo do espaço vetorial $R(P(s))$ sobre o espaço vetorial $R(P(t))$. Neste caso, o isomorfismo inverso é indicado por $U(s, t): R(P(t)) \rightarrow R(P(s))$.

(iii) Verificam-se as seguintes estimativas

$$
\begin{array}{r}
\|U(t, s)(I-P(s))\|_{\mathcal{L}(X)} \leqslant K e^{-\beta(t-s)} \text { sempre que } t \geqslant s \\
\|U(t, s) P(s)\|_{\mathcal{L}(X)} \leqslant K e^{\beta(t-s)}, \text { sempre que } t \leqslant s .
\end{array}
$$

Por outro lado, se $\{T(t, s): t \geq s\}$ é um processo de evolução em $X$, gerado por uma equação semilinear do tipo

$$
\left\{\begin{array}{l}
\dot{x}=M x+G(t, x) t>s, \\
x(s)=x_{0} \in X
\end{array}\right.
$$


onde $M: D(M) \subset X \rightarrow X$ é um gerador de $C_{0}$-semigrupo em $X$ e $G: \mathbb{R} \times X \rightarrow$ $X$ é uma certa aplicação possuindo derivada parcial de primeira ordem com relação à segunda variável contínua. Uma solução global $\xi: \mathbb{R} \rightarrow X$ deste processo diz-se uma solução global hiperbólica quando o processo $\{U(t, s): t \geq s\}$ associado à linearização de (8.1.5) em torno de $\xi$, ou seja, o problema linear não autônomo dado por

$$
\left\{\begin{array}{l}
\dot{x}=M x+G_{x}(t, \xi(t)) t>s, \\
x(s)=x_{0} \in X
\end{array}\right.
$$

possui dicotomia exponencial.

Para levar a cabo o problema proposto acima, necessitamos do próximo teorema, que foi provado em [11] e que a seguir apenas enunciamos sem demonstração.

Teorema 8.1.4. Sejam, para $\eta \in(0,1], f_{\eta}: \mathbb{R} \times Y \rightarrow Y$ uma aplicação possuindo devirada parcial com respeito à segunda variável contínua em $\mathbb{R} \times Y$ e $f_{0}: Y \rightarrow Y$ uma aplicação continuamente diferenciável.

Consideremos os problemas

$$
\left\{\begin{array}{l}
\dot{y}=B y+f_{\eta}(t, y) t>s \\
y(s)=y_{0}
\end{array}\right.
$$

$e$

$$
\left\{\begin{array}{l}
\dot{y}=B y+f_{0}(y) t>s \\
y(s)=y_{0}
\end{array} .\right.
$$

Suponhamos que as aplicações $f_{\eta}: \mathbb{R} \times Y \rightarrow Y$, para $\eta \in(0,1]$, e $f_{0}: Y \rightarrow Y$ sejam tais que (8.1.6) e (8.1.7) gerem, respectivamente, os processos de evolução $\left\{T_{\eta}(t, s)\right.$ : $t \geq s\}, \eta \in(0,1]$, e o semigrupo $\left\{T_{0}(t): t \geq 0\right\}$.

Suponhamos, além disso, que $\left\{T_{0}(t): t \geq 0\right\}$ possua atrator global $\mathcal{A}$ e somente um número finito de pontos de equilíbrio $\mathcal{E}=\left\{y_{1}^{*}, \cdots, y_{n}^{*}\right\}$ sendo todos eles hiperbólicos.

Se para cada $r>0$

$$
\lim _{\eta \rightarrow 0^{+}} \sup _{t \in \mathbb{R}} \sup _{\|y\|_{Y} \leq r}\left\{\left\|f_{\eta}(t, y)-f_{0}(y)\right\|_{Y}+\left\|\left(f_{\eta}\right)_{y}(t, y)-f_{0}^{\prime}(y)\right\|_{\mathcal{L}(Y)}\right\}=0
$$

então existe $\eta_{0} \in(0,1]$ tal que para cada $\eta \leq \eta_{0}$ existem $\xi_{i, \eta}^{*}: \mathbb{R} \rightarrow Y, i=1, \cdots, n$, soluções globais hiperbólicas para (8.1.6) com

$$
\lim _{\eta \rightarrow 0^{+}} \sup _{t \in \mathbb{R}}\left\|\xi_{i, \eta}^{*}(t)-y_{i}^{*}\right\|_{Y}=0
$$

qualquer que seja $i=1, \cdots, n$. 
Observação 8.1.5. Neste ponto, é importante compararmos as conclusões deste último teorema com a hipótese (c) do Teorema 7.5.1.

Agora, seja $f: \mathbb{R} \times Y \rightarrow Y$ uma aplicação tal que o problema semilinear

$$
\left\{\begin{array}{c}
\dot{y}=B y+f(t, y) t>s \\
y(s)=y_{0} \in Y
\end{array},\right.
$$

gere um processo de evolução $\{T(t, s): t \geq s\}$ possuindo atrator pullback $\{\mathcal{A}(t): t \in \mathbb{R}\}$ com $\overline{\bigcup_{t \in \mathbb{R}} \mathcal{A}(t)}$ compacto.

Definindo, para cada $\nu>0$ e $\mu>0$, as aplicações $f_{\nu}, f^{\mu}: \mathbb{R} \times Y \rightarrow Y$ por

$$
f_{\nu}(t, y):=\left\{\begin{array}{l}
f(t, y), \text { se } t \leq-\nu \\
f(-\nu, y), \text { se } t>-\nu
\end{array}\right.
$$

e

$$
f^{\mu}(t, y):=\left\{\begin{array}{l}
f(t, y), \text { se } t \geq \mu \\
f(\mu, y), \text { se } t<\mu
\end{array} .\right.
$$

Diz-se que $f$ é compatível à esquerda (respectivamente, à direita) com respeito ao sistema (8.1.8) quando existir $\nu_{0}>0$ (resp. $\mu_{0}>0$ ) tal que para todo $\nu \geq \nu_{0}$ (resp. $\mu \geq \mu_{0}$ ) o problema

$$
\left\{\begin{array}{l}
\dot{y}=B y+f_{\nu}(t, y) t>s \\
y(s)=y_{0}
\end{array},\right.
$$

(resp. o problema

$$
\left\{\begin{array}{l}
\dot{y}=B y+f^{\mu}(t, y) t>s \\
y(s)=y_{0} \in Y
\end{array}\right)
$$

gere um processo de evolução $\left\{T_{\nu}(t, s): t \geq s\right\}$ possuindo atrator pullback $\left\{\mathcal{A}_{\nu}(t): t \in\right.$ $\mathbb{R}\}$ com $\overline{\bigcup_{\nu \geq \nu_{0}} \bigcup_{\in \mathbb{R}} \mathcal{A}_{\nu}(t)}$ compacto em $Y$ (resp. gere um processo de evolução $\left\{T^{\mu}(t, s)\right.$ : $t \geq s\}$ possuindo atrator pullback $\left\{\mathcal{A}^{\mu}(t): t \in \mathbb{R}\right\}$ com $\overline{\bigcup_{\mu \geq \mu_{0}} \bigcup_{t \in \mathbb{R}} \mathcal{A}^{\mu}(t)}$ compacto em $Y$ )

Com o que acabamos de apresentar acima podemos demonstrar o lema a seguir. É ele quem torna possível provar que o problema dado em 8.1.2 é de tipo gradiente. Em sua demonstração, mais uma vez, utilizamos o Teorema 7.5.1 sobre perturbações não autônomas pequenas de um semigrupo gradiente.

Lema 8.1.6. Consideremos fixadas as condições postas acima. Suponhamos que o sistema (8.1.1) é tal que a equação desacoplada $\dot{x}=A x+g(x)$ satisfaça a condição de que todas as suas soluções globais limitadas convirjam para pontos estacionários tanto 
quando $t \rightarrow-\infty$ quanto quando $t \rightarrow \infty$ e que, além disso, para cada um de seus pontos estacionários $x^{*} \in \mathcal{S}$ o problema semilinear autônomo

$$
\left\{\begin{array}{l}
\dot{y}=B y+f\left(x^{*}, y\right) t>s \\
y(s)=y_{0} \in Y
\end{array}\right.
$$

gere um semigrupo de tipo gradiente $\left\{T_{x^{*}}(t): t \geq 0\right\}$ com respeito ao conjunto finito de pontos estacionários $\mathcal{E}_{x^{*}}$, todos eles hiperbólicos, e possuindo atrator global $\mathcal{A}_{x^{*}} e$

Seja $\xi=(\varphi, \psi): \mathbb{R} \rightarrow X \times Y$ uma solução global limitada de (8.1.2) de maneira que, escrevendo por simplicidade, $f(t, y):=f(\varphi(t), y),(t, y) \in \mathbb{R} \times Y$, o problema não autônomo

$$
\left\{\begin{array}{c}
\dot{y}=B y+f(t, y) t>s \\
y(s)=y_{0} \in Y
\end{array}\right.
$$

(do qual $\psi: \mathbb{R} \rightarrow Y$ é uma solução) gere um processo de evolução $\{T(t, s): t \geq s\}$ possuindo atrator pullback $\{\mathcal{A}(t): t \in \mathbb{R}\}$, com $\bigcup_{t \in \mathbb{R}} \mathcal{A}(t)$ relativamente compacto, e que $f=f(t, y)$ seja compatível à direita e à esquerda com respeito ao sistema (8.1.10).

Da hipótese anterior, existem equilíbrios $x_{-}^{*}, x_{+}^{*}$ em $\mathcal{S}$ tais que

$$
\lim _{t \rightarrow-\infty}\left\|\varphi(t)-x_{-}^{*}\right\|_{X}=0 e \lim _{t \rightarrow \infty}\left\|\varphi(t)-x_{+}^{*}\right\|_{X}=0 .
$$

Se para cada $r>0$,

$$
\lim _{t \rightarrow-\infty} \sup _{\|y\|_{Y} \leq r}\left\{\left\|f(t, y)-f\left(x_{-}^{*}, y\right)\right\|_{Y}+\left\|f_{y}(t, y)-f_{y}\left(x_{-}^{*}, y\right)\right\|_{\mathcal{L}(Y)}\right\}=0
$$

$e$

$$
\lim _{t \rightarrow \infty} \sup _{\|y\|_{Y} \leq r}\left\{\left\|f(t, y)-f\left(x_{+}^{*}, y\right)\right\|_{Y}+\left\|f_{y}(t, y)-f_{y}\left(x_{+}^{*}, y\right)\right\|_{\mathcal{L}(Y)}\right\}=0
$$

então existem equilíbrios $y_{-}^{*} \in \mathcal{E}_{x_{-}^{*}}$ e $y_{+}^{*} \in \mathcal{E}_{x_{+}^{*}}$ tais que, pondo $z_{-}^{*}:=\left(x_{-}^{*}, y_{-}^{*}\right)$ e $z_{+}^{*}:=\left(x_{+}^{*}, y_{+}^{*}\right)$, tem-se

$$
\lim _{t \rightarrow-\infty}\left\|\xi(t)-z_{-}^{*}\right\|_{X \times Y}=0 e \lim _{t \rightarrow \infty}\left\|\xi(t)-z_{+}^{*}\right\|_{X \times Y}=0 .
$$

Demonstração: Analisemos apenas o caso $t \rightarrow-\infty$, porque o caso onde se faz $t$ tender a $\infty$ se demonstra de modo semelhante.

Com efeito, definindo, para cada $\nu>0$, a aplicação $f_{\nu}: \mathbb{R} \times Y \rightarrow Y$ como em (8.1.9) e considerando a família de problemas semilineares

$$
\left\{\begin{array}{l}
\dot{y}=B y+f_{\nu}(t, y) t>s \\
y(s)=y_{0}
\end{array}, \nu>0\right.
$$

segue-se, pela hipótese de compatibilidade, a existência de um real positivo $\nu_{0}$ tal que cada $f_{\nu}$, com $\nu \geq \nu_{0}$, herda de $f$ as propriedades necessárias para a geração de um 
processo de evolução $\left\{T_{\nu}(t, s): t \geq s\right\}$, possuindo atrator pullback $\left\{\mathcal{A}_{\nu}(t): t \in \mathbb{R}\right\}$ com $\bigcup_{\nu \geq \nu^{\prime} t \in \mathbb{R}} \mathcal{A}_{\nu}(t)$ compacto em $Y$.

Agora, recordando que os processos de evolução gerados por problemas semilineares são dados pela fórmula da variação das constantes, não é difícil ver que para $t \leq-\nu$ tem-se $T_{\nu}(t, s)=T(t, s)$ toda vez que $s \leq t$, donde, se $\zeta: \mathbb{R} \rightarrow Y$ é uma solução global para $\{T(t, \tau): t \geq \tau\}$ temos $\zeta(t)=T(t, s) \zeta(s)=T_{\nu}(t, s) \zeta(s)$ sempre que $s \leq t \leq-\nu$. Assim, definindo, para cada $\nu \geq \nu_{0}, \zeta_{\nu}: \mathbb{R} \rightarrow Y$ por

$$
\zeta_{\nu}(t):=\left\{\begin{array}{l}
\zeta(t), \text { se } t \leq-\nu \\
T_{\nu}(t,-\nu) \zeta(-\nu), y, \text { se } t>-\nu
\end{array}\right.
$$

resulta que $\zeta_{\nu}: \mathbb{R} \rightarrow Y$ é uma solução global para $\left\{T_{\nu}(t, s): t \geq s\right\}$.

Por um lado, é fácil ver, usando (8.1.11), que para todo $r>0$ tem-se

$$
\lim _{\nu \rightarrow \infty} \sup _{t \in \mathbb{R}} \sup _{\|y\|_{Y} \leq r}\left\{\left\|f_{\nu}(t, y)-f\left(x_{-}^{*}, y\right)\right\|_{Y}+\left\|\left(f_{\nu}\right)_{y}(t, y)-f_{y}\left(x_{-}^{*}, y\right)\right\|_{\mathcal{L}(Y)}\right\}=0 .
$$

Então, escrevendo $\mathcal{E}_{x_{-}^{*}}=\left\{y_{1,-}^{*}, y_{2,-}^{*}, \cdots, y_{n,-}^{*}\right\}$, do Teorema 8.1.4 segue a existência de $\nu_{1}>0$ tal que, para cada $\nu \geq \nu_{1}$ o problema (8.1.13) possui $n$ soluções globais hiperbólicas $\xi_{i, \nu}^{*}: \mathbb{R} \rightarrow Y, i=1, \cdots, n$, satisfazendo, para cada $i=1, \cdots, n$, a convergência

$$
\lim _{\nu \rightarrow \infty} \sup _{t \in \mathbb{R}}\left\|\xi_{i, \nu}^{*}(t)-y_{i,-}^{*}\right\|_{Y}=0 .
$$

Por outro, sabemos também que, de (8.1.15) e novamente pelo fato de que os processos gerados por problemas semilineares vêm dados pela fórmula da variação das constantes, para cada $R>0$ e cada compacto $K \subset Y$, vale

$$
\limsup _{\nu \rightarrow \infty} \sup _{s \in \mathbb{R}} \sup _{t \in[0, R]}\left\|T_{\nu \in K}(t+s, s) y-T_{x_{-}^{*}}(t) y\right\|_{Y}=0 .
$$

Portanto, usando este último fato em conjunto com a hiperbolicidade das soluções ${ }^{1}$ $\xi_{i, \nu}^{*}: \mathbb{R} \rightarrow Y, i=1, \cdots, n$, estamos com todas as hipóteses do Teorema 7.5.1 satisfeitas, consequentemente existe $\nu_{0} \geq \nu_{1}$ tal que cada $\left\{T_{\nu}(t, s): t \geq s\right\}$, com $\nu \geq \nu_{0}$, é um processo de evolução de tipo gradiente com respeito ao conjunto finito de soluções isoladas $\left\{\xi_{1, \nu}^{*}, \cdots, \xi_{n, \nu}^{*}\right\}$.

Logo, como $\psi: \mathbb{R} \rightarrow Y$ é solução limitada de $\{T(t, s): t \geq s\}$, considerando, de acordo com (8.1.14), as soluções $\psi_{\nu}: \mathbb{R} \rightarrow Y$ para $\left\{T_{\nu}(t, s): t \geq s\right\}$ associadas a $\psi: \mathbb{R} \rightarrow Y$, temos que cada $\psi_{\nu}$ é uma solução tal que para todo real $t, \psi_{\nu}(t) \in \mathcal{A}_{\nu}(t)$,

\footnotetext{
${ }^{1} \mathrm{~A}$ hiperbolicidade das soluções $\xi_{i, \nu}^{*}: \mathbb{R} \rightarrow Y, i=1, \cdots, n$, é utilizada aqui para se estabelecer a hipótese $(e)$ do Teorema 7.5.1. Deve-se, para isso, recordar que na prova do Teorema da variedade instável, quando uma solução permanece em uma certa vizinhança da solução hiperbólica, dita solução deve convergir para a hiperbólica, devido a um argumento de unicidade de ponto fixo. Confira [14].
} 
porque são limitadas para trás, uma vez que coincidem com $\psi$ para $t \leq-\nu$, donde existe (um único) índice $i_{0} \in\{1, \cdots, n\}$ tal que para $\nu$ suficientemente grande tem-se

$$
\lim _{t \rightarrow-\infty}\left\|\psi(t)-\xi_{i_{0}, \nu}^{*}(t)\right\|_{Y}=\lim _{t \rightarrow-\infty}\left\|\psi_{\nu}(t)-\xi_{i_{0}, \nu}^{*}(t)\right\|_{Y}=0
$$

O que em conjunto com (8.1.16) nos dá, imediatamente, que

$$
\lim _{t \rightarrow-\infty}\left\|\psi(t)-y_{i_{0},-}^{*}\right\|_{Y}=0
$$

terminando a demonstração.

Podemos agora provar que, sob as condições impostas no lema acima, o problema semilinear (8.1.2) é de tipo gradiente.

Teorema 8.1.7. Se o problema semilinear (8.1.2) satisfaz as hipóteses do Lema 8.1.6, então o semigrupo $\{T(t): t \geq 0\}$, a ele associado, é de tipo gradiente com respeito a $\mathcal{E}$.

Demonstração: De fato, o Lema 8.1.6 diz exatamente que $\{T(t): t \geq 0\}$ satisfaz a condição $\left(G 1^{\prime}\right)$ da Definição 8.1.1.

Para provar $\left(G 2^{\prime}\right)$ suponhamos, caso contrário, que existam $\left\{\xi_{i}=\left(\varphi_{i}, \psi_{i}\right): \mathbb{R} \rightarrow\right.$ $X \times Y: i=1, \cdots, k\}$ soluções globais e pontos de equilíbrio $\left\{z_{i}^{*}=\left(x_{i}^{*}, y_{i}^{*}\right): i=\right.$ $1, \cdots, k\} \subset \mathcal{E}$ constituindo uma estrutura homoclínica associada a $\mathcal{E}$.

Observemos, primeiramente, que cada $\varphi_{i}: \mathbb{R} \rightarrow X$ é solução global de $\dot{x}=A x+g(x)$ e, por isso, cada $\psi_{i}: \mathbb{R} \rightarrow Y$ é solução da equação não autônoma $\dot{y}=B y+f\left(\varphi_{i}(t), y\right)$ e verificam-se as convergências:

$$
\begin{aligned}
& \lim _{t \rightarrow-\infty}\left\|\varphi_{i}(t)-x_{i}^{*}\right\|_{X}=0, \lim _{t \rightarrow \infty}\left\|\varphi_{i}(t)-x_{i+1}^{*}\right\|_{X}=0, \\
& \lim _{t \rightarrow-\infty}\left\|\psi_{i}(t)-y_{i}^{*}\right\|_{Y}=0 \text { e } \lim _{t \rightarrow \infty}\left\|\psi_{i}(t)-y_{i+1}^{*}\right\|_{Y}=0,
\end{aligned}
$$

para $i=1,2, \cdots, k \operatorname{com} x_{k+1}^{*}:=x_{1}^{*}$ e $y_{k+1}^{*}:=y_{1}^{*}$.

Temos assim dois casos a considerar:

Caso 1: Existe algum índice $i_{0} \in\{1, \cdots, k\}$ tal que $\varphi_{i_{0}}: \mathbb{R} \rightarrow X$ é solução não constante de $\dot{x}=A x+g(x)$. Daí segue-se facilmente que $\varphi_{i_{0}}: \mathbb{R} \rightarrow X$ pertence a alguma estrutura homoclínica no atrator de $\dot{x}=A x+g(x)$ associada a $\mathcal{S}$, o que contradiz o fato de que $\dot{x}=A x+g(x)$ é um sistema de tipo gradiente.

Caso 2: Para todo $i \in\{1, \cdots, k\}$ as soluções $\varphi_{i}: \mathbb{R} \rightarrow X$ são todas soluções de equilíbrio. Neste caso, necessariamente, todos os pontos $x_{i}^{*}$ 's devem ser iguais a um certo $x^{*} \in \mathcal{S}$ fixo e, pelas covergências (8.1.17), $\varphi_{i}(t)=x^{*}$ para todo $i=1, \cdots, k$ e para todo $t \in \mathbb{R}$. Consequentemente, todas as aplicações $\psi_{i}: \mathbb{R} \rightarrow Y$ são soluções 
da mesma equação $\dot{y}=B y+f\left(x^{*}, y\right)$ e, portanto, devem formar, juntamente com os equilíbrios $\left\{y_{1}^{*}, \cdots, y_{k}^{*}\right\}$ uma estrutura homoclínica no atrator de $\dot{y}=B y+f\left(x^{*}, y\right)$ associada a $\mathcal{E}_{x^{*}}$, o que contradiz a hipótese de que $\dot{y}=B y+f\left(x^{*}, y\right)$ é de tipo gradiente com respeito a $\mathcal{E}_{x^{*}}$, completando assim a demonstração do teorema.

Corolário 8.1.8. Se o problema semilinear (8.1.2) satisfaz as hipóteses do Lema 8.1.6, então o atrator $\mathcal{A}$ do semigrupo $\{T(t): t \geq 0\}$, a ele associado, se escreve como a reunião dos conjuntos instáveis de seus pontos estacionários.

Demonstração: Com efeito, usando a conclusão do Lema 8.1.6 obtém-se que toda solução limitada de (8.1.2) converge, quando $t \rightarrow-\infty$, para uma solução de equilíbrio e o corolário fica demonstrado.

Corolário 8.1.9. Se o problema semilinear (8.1.2) satisfaz as hipóteses do Lema 8.1.6 com conjunto $\mathcal{E}$ de pontos estacionários finito, então o semigrupo $\{T(t): t \geq 0\}, a$ ele associado, é gradiente com respeito a $\mathcal{E}$.

Demonstração: Com efeito, pelo Teorema 8.1.7, $\{T(t): t \geq 0\}$ é de tipo gradiente no sentido da Definición 8.1.1. Contudo, como estamos supondo que seu conjunto de pontos de equilíbrio $\mathcal{E}$ é finito, resulta que $\{T(t): t \geq 0\}$ é de tipo gradiente segundo a Definição 3.1 .1 e então a conclusão do corolário segue-se por aplicação direta do Teorema 4.4.1.

\subsection{A desigualdade de Lojasiewicz-Simon}

Dada uma solução global de um semigrupo, a presente seção tem como objetivo fornecer uma importante ferramenta que garanta sua covergência, no "passado" e no "futuro", para pontos de equilíbrio, portanto, fornecer uma condição para que uma das hipóteses do Teorema 8.1.7 esteja setisfeita. Em termos mais precisos, fornecemos condições para que esteja satisfeita a "Desigualdade de Lojasiewicz-Simon" para problemas gradientes abstratos em espaços de Hilbert.

Devemos observar que uma condição deste tipo é importante porque, como vimos na Proposição 2.2.2 do Capítulo 2, no caso em que o conjunto de equilíbrios é finito, a hipótese de que o semigrupo associado à equação seja um semigrupo gradiente e o fato de que o conjunto $\omega$-limite de um ponto é conexo, obrigam às soluções com órbitas relativamente compactas a terem conjunto $\omega$-limite unitário e, portanto, formado por um único ponto estacionário. Não obstante, no caso em que o conjunto de equilíbrios 
não é finito a convergência de tais soluções para um (único) ponto de equilíbrio pode não ocorrer.

Sejam, então, $V:=\left(V,(\cdot, \cdot)_{V}\right)$ e $H:=\left(H,(\cdot, \cdot)_{H}\right)$ espaços de Hilbert reais tais que $V$ é um subespaço denso de $H$ e a inclusão de $V$ em $H$ é compacta. Como de costume, identificamos $H$ com seu dual topológico $H^{\prime}$ e indicamos por $V^{\prime}$ o dual topológico de $V$ (os quais não se identificam), de modo a termos a cadeia de inclusões contínuas e densas:

$$
V \hookrightarrow H \hookrightarrow V^{\prime}
$$

Teorema 8.2.1 (Desigualdade de Lojasiewicz-Simon). Seja $G \in C^{2}(V ; \mathbb{R})$ e indiquemos sua aplicação derivada (no sentido de Fréchet) por $M:=G^{\prime}=\nabla G: V \rightarrow V^{\prime}$.

Seja $\varphi \in V$ uma solução da equação $M(u)=0$ satisfazendo as seguintes duas condições:

(i) A linearização, $L:=M^{\prime}(\varphi) \in \mathcal{L}\left(V, V^{\prime}\right)$, de $M$ em torno de $\varphi$ se escreve como:

$$
L=\Lambda+B
$$

onde $\Lambda: V \rightarrow V^{\prime}$ é um isomorfismo e $B: V \rightarrow V^{\prime}$ um operador compacto.

(ii) Se o núcleo de $L, \operatorname{ker}(L)$, é não trivial, suponhamos que o operador $L: V \subset V^{\prime} \rightarrow$ $V^{\prime}$, visto como operador ilimitado em $V^{\prime}$ com domínio em $V$, possua conjunto resolvente não vazio e, considerando-se a projeção $\Pi: H \rightarrow H$ definida por

$$
\Pi u:=\frac{1}{2 \pi i} \int_{\gamma}(\lambda-L)^{-1} u d \lambda, u \in H
$$

onde $\gamma:[0,1] \rightarrow \rho(L)$ é uma curva fechada, simples e suave com $\int_{\gamma} \frac{1}{\zeta} d \zeta=2 \pi i$, cuja imagem $R(\Pi)$ tem dimensão finita ${ }^{2} d:=\operatorname{dim}(R(\Pi)) \in \mathbb{N}$, suponhamos que exista um conjunto aberto $U \subset \mathbb{R}^{d}$ e um homeomorfismo $h: U \rightarrow h(U)$, tal que $\varphi \in h(U) \subset M^{-1}(0)$.

Então a desigualdade de L Lojasiewicz-Simon está satisfeita em $\varphi$ com o melhor expoente, isto é, existem $\sigma>0$ e $c>0$ tais que para todo $u \in V$ com $\|u-\varphi\|_{V}<\sigma$ tem-se

$$
|G(u)-G(\varphi)| \leq c\|M(u)\|_{V^{\prime}}^{2}
$$

Demonstração: A prova está dividida em dois casos:

Caso 1: Neste caso supomos que $\operatorname{ker}(L)=\{0\}$, o que corresponde ao caso em que, devido à hipotese $(i)$ e a Alternativa de Fredholm, $L: V \rightarrow V^{\prime}$ é um isomorfismo.

\footnotetext{
${ }^{2}$ Lembremos que a imagem de uma projeção compacta tem dimensão finita.
} 
Fazendo a expansão de Taylor de segunda ordem de $G$ em torno de $\varphi$, para todo $u$ obtém-se

$$
\begin{aligned}
G(u)-G(\varphi) & =\langle M(\varphi), u-\varphi\rangle_{V^{\prime}, V}+\left\langle G^{\prime \prime}(\varphi)(u-\varphi), u-\varphi\right\rangle_{V^{\prime}, V}+o\left(\|u-\varphi\|_{V}^{2}\right) \\
& =\left\langle G^{\prime \prime}(\varphi)(u-\varphi), u-\varphi\right\rangle_{V^{\prime}, V}+o\left(\|u-\varphi\|_{V}^{2}\right)
\end{aligned}
$$

o que nos dá

$$
|G(u)-G(\varphi)| \leq C_{1}\|u-\varphi\|_{V}^{2},
$$

para $u$ suficientemente próximo de $\varphi$ e uma certa constante positiva $C_{1}$.

Por outro lado, fazendo agora o desenvolvimento de Taylor de primeira ordem para $M=G^{\prime}$ em torno de $\varphi$ temos

$$
M(u)=M(\varphi)+L(u-\varphi)+o\left(\|u-\varphi\|_{V^{\prime}}\right)=L(u-\varphi)+o\left(\|u-\varphi\|_{V}\right),
$$

donde obtém-se

$$
u-\varphi=L^{-1}[M(u)]+o\left(\|u-\varphi\|_{V}\right) .
$$

Então, para $u$ suficientemente próximo de $\varphi$ temos

$$
\|u-\varphi\|_{V} \leq C_{2}\|M(u)\|_{V^{\prime}}
$$

para alguma constante $C_{2}>0$. Logo, levando esta desigualdade em (8.2.18), obtemos a existência de $\sigma>0$ tal que $\|u-\varphi\|_{V}<\sigma \Rightarrow|G(u)-G(\varphi)| \leq C\|M(u)\|_{V^{\prime}}^{2}$, para uma certa constante $C>0$, terminando a demonstração do primeiro caso.

Caso 2: Suponhamos agora que $\operatorname{ker}(L) \neq\{0\}$. Simplesmente fazendo uma mudança de variáveis é suficiente estudarmos o caso em que $\varphi=0$ e $G(\varphi)=0$, pois, de outra forma, definimos $G_{0}: V \rightarrow \mathbb{R}$ pondo $G_{0}(u):=G(u+\varphi)-G(\varphi)$ e daí é claro que o resultado é certo para $G_{0}$ se, e somente se, o é para $G$.

Nestas condições, seja $\mathcal{L}: V \rightarrow V^{\prime}$ a aplicação linear dada por

$$
\mathcal{L} u:=\Pi u+L u, u \in V \text {. }
$$

Afirmamos que $\mathcal{L}$ é injetiva. Com efeito, seja $u_{0} \in V$ tal que $0=\mathcal{L} u_{0}=\Pi u_{0}+L u_{0}$. Como $L$ comuta com $\Pi$ segue-se que $0=\Pi^{2} u_{0}+\Pi L u_{0}=\Pi u_{0}+L \Pi u_{0}$. Seja $n_{0} \in \mathbb{N}$ o menor inteiro positivo tal que $R(\Pi)=\operatorname{ker}\left(L^{n_{0}}\right) .{ }^{3}$ Deste fato e o de que $\Pi u_{0}=-L u_{0}$

\footnotetext{
${ }^{3}$ Para verificar esta afirmação, observemos que $\operatorname{ker}\left(L^{n}\right) \subset R(\Pi)$ para todo natural $n$ pois, primeiramente, de acordo com a decomposição espectral tem-se $\operatorname{ker}(L) \subset R(\Pi)$. Agora se $u \in \operatorname{ker}\left(L^{2}\right) \backslash R(\Pi)$, tem-se que $u=u_{1}+u_{2}$, com $u_{1} \in R(\Pi), u_{2} \in \operatorname{ker}(\Pi)$ e $u_{2} \neq 0$. Daí vem que $L^{2} u_{1}=-L^{2} u_{2}$ e como $\Pi$ comuta com $L$ devemos ter $L^{2} u_{2}=0$, ou seja $L u_{2} \in \operatorname{ker}(L)$ com $L u_{2} \neq 0$. Como $\left.L\right|_{\operatorname{ker}(\Pi)}: \operatorname{ker}(\Pi) \rightarrow \operatorname{ker}(\Pi)$, resulta que $L u_{2} \in \operatorname{ker}(\Pi)$, mas isso contradiz que $\left.L\right|_{\operatorname{ker}(\Pi)}$ é um isomorfismo. A indução esta clara do que acabamos de fazer e o fato de que a mesma deve parar em um número finito de passos segue do fato de que $\operatorname{dim}(R(\Pi))=d<\infty$.
} 
vemos que $L^{n_{0}-1} \Pi u_{0}=-L^{n_{0}} \Pi u_{0}=0$ e $L^{n_{0}-1} \Pi u_{0}=0$. Realizando um argumento de indução obteremos $\Pi u_{0}=0$ e consequentemente $L u_{0}=-\Pi u_{0}=0$ donde $u_{0} \in \operatorname{ker}(L) \subset$ $R(\Pi)$. Portanto $u_{0}=\Pi u_{0}=0$ e a injetividade de $\mathcal{L}$ segue.

Considereremos agora a aplicação $\mathcal{N}: V \rightarrow V^{\prime}$ definida por

$$
\mathcal{N} u:=\Pi u+M(u), u \in V
$$

Das hipóteses sobre $G$ conclui-se que $\mathcal{N}$ é de classe $C^{1} \operatorname{com} \mathcal{N}^{\prime}(0)=\Pi+M^{\prime}(0)=\mathcal{L}$. Logo, pelo Teorema da Aplicação Inversa, existem conjuntos abertos $W_{1}(0) \subset V$ com $0 \in W_{1}(0)$ e $W_{2}(0) \subset V^{\prime}$ com $0 \in W_{2}(0)$, tais que $\mathcal{N}$ é um difeomorfismo de $W_{1}(0)$ sobre $W_{2}(0)$. Assim, existe a aplicação inversa de $\mathcal{N}: W_{1}(0) \rightarrow W_{2}(0)$, que denotamos por $\Psi: W_{2}(0) \rightarrow W_{1}(0)$, e que é também de classe $C^{1}$.

Diminuindo os conjuntos abertos $W_{1}(0)$ e $W_{2}(0)$, se necessário, e utilizando-se a desigualdade do valor médio podemos supor que

$$
\begin{aligned}
& \left\|\Psi\left(g_{1}\right)-\Psi\left(g_{2}\right)\right\|_{V} \leq C_{1}\left\|g_{1}-g_{2}\right\|_{V^{\prime}} \quad \forall g_{1}, g_{2} \in W_{2}(0), \\
& \|M(u)-M(v)\|_{V^{\prime}} \leq C_{2}\|u-v\|_{V} \quad \forall u, v \in W_{1}(0),
\end{aligned}
$$

para certas constantes positivas $C_{1}$ e $C_{2}$.

Por outro lado, consideremos uma base ortonormal $\left\{\varphi_{1}, \cdots, \varphi_{d}\right\}$ para $R(\Pi)=$ $\operatorname{ker}\left(L^{n_{0}}\right)$ com respeito ao produto escalar de $H$ e definamos a aplicação $f: \mathbb{R}^{d} \rightarrow V^{\prime}$ pondo

$$
f(\xi):=\sum_{j=1}^{d} \xi_{j} \varphi_{j}, \text { para } \xi=\left(\xi_{1}, \ldots, \xi_{d}\right) \in \mathbb{R}^{d} .
$$

É claro que $f$ é um isomorfismo entre $\mathbb{R}^{d}$ e $\operatorname{ker}\left(L^{n_{0}}\right)$. Então

$$
\widetilde{W}_{2}(0):=f^{-1}\left(W_{2}(0)\right)=\left\{\xi \in \mathbb{R}^{d}: f(\xi) \in W_{2}(0)\right\}
$$

é um conjunto aberto de $\mathbb{R}^{d}$ que contém o zero. Consideremos também a função real $\Gamma: \widetilde{W}_{2}(0) \rightarrow \mathbb{R}$ dada por

$$
\Gamma(\xi):=(G \circ \Psi \circ f)(\xi), \xi \in \widetilde{W}_{2}(0) .
$$

Obviamente, $\Gamma$ é de classe $C^{1}$ em $\widetilde{W}_{2}(0)$.

Agora definamos

$$
\widetilde{W}_{1}(0):=\left\{u \in W_{1}(0): \Pi u \in W_{2}(0)\right\}
$$

Não é difícil ver, usando que $\Pi^{-1}\left(W_{2}(0)\right)$ é aberto em $H$ e a inclusão contínua $V \hookrightarrow H$, que o conjunto $\widetilde{W}_{1}(0)$ é aberto em $V$. 
Nestas condições, dado $u \in \widetilde{W}_{1}(0)$ consideremos $\xi \in \widetilde{W}_{2}(0)$ tal que $f(\xi)=\Pi u \in$ $W_{2}(0)$.

Afirmamos que existe uma constante $C>0$, independente de $u$ e de $\xi$, tal que

$$
|G(u)-\Gamma(\xi)| \leq C\|M(u)\|_{V^{\prime}}^{2}
$$

De fato,

$$
\begin{aligned}
|G(u)-\Gamma(\xi)| & =|G(u)-G(\Psi(f(\xi)))|=\left|\int_{0}^{1} \frac{d}{d t}\{G(u+t[\Psi(f(\xi))-u])\} d t\right| \\
& =\left|\int_{0}^{1}\langle M(u+t[\Psi(f(\xi))-u]), \Psi(f(\xi))-u\rangle d t\right| \\
& \leq \int_{0}^{1}|\langle M(u+t[\Psi(f(\xi))-u]), \Psi(f(\xi))-u\rangle| d t \\
& \leq \int_{0}^{1}\|M(u+t[\Psi(f(\xi))-u])\|_{V^{\prime}}\|\Psi(f(\xi))-u\|_{V} d t \\
& =\|\Psi(f(\xi))-u\|_{V} \int_{0}^{1}\|M(u+t[\Psi(f(\xi))-u])\|_{V^{\prime}} d t .
\end{aligned}
$$

Assim, usando (8.2.19) temos

$$
\begin{aligned}
\|M(u+t[\Psi(f(\xi))-u])\|_{V^{\prime}} & =\|M(u+t[\Psi(f(\xi))-u])-M(u)+M(u)\|_{V^{\prime}} \\
& \leq\|M(u)\|_{V^{\prime}}+C_{2}\|t[\Psi(f(\xi))-u]\|_{V^{\prime}} .
\end{aligned}
$$

Aplicando pois esta desigualdade à anterior deduz-se que

$$
|G(u)-\Gamma(\xi)| \leq\|\Psi(f(\xi))-u\|_{V} \int_{0}^{1}\left\{\|M(u)\|_{V^{\prime}}+t C_{2}\|[\Psi(f(\xi))-u]\|_{V^{\prime}}\right\} d t
$$

Novamente de (8.2.19) conclui-se que

$$
\|\Psi(f(\xi))-u\|_{V}=\|\Psi(f(\xi))-\Psi(\Pi u+M(u))\|_{V} \leq C_{1}\|M(u)\|_{V^{\prime}}
$$

E isso juntamente com (8.2.21) nos leva a (8.2.20).

Observemos que, levando em conta que (8.2.20) é certo, nossa demonstração resultará completa se provarmos que $\Gamma(\xi)=0$ para $\xi$ suficientemente pequeno.

Com efeito, para cada $k=1, \ldots, d$ pela regra da cadeia temos

$$
\frac{\partial \Gamma}{\partial \xi_{k}}(\xi)=\left\langle M(\Psi(f(\xi))), \Psi^{\prime}(f(\psi)) \varphi_{k}\right\rangle=\left(M(\Psi(f(\xi))), \Psi^{\prime}(f(\psi)) \varphi_{k}\right)_{H},
$$

onde a última igualdade conclui-se do fato de que $M(\Psi(f(\xi)))=f(\xi)-\Pi(\Psi(f(\xi))) \in$ $N\left(L^{n_{0}}\right) \subset H \hookrightarrow V^{\prime}$. 
Como $M(\Psi(f(\xi))) \in N\left(L^{n_{0}}\right)$, podemos escrevê-lo como combinação linear dos elementos da base $\left\{\varphi_{1}, \cdots, \varphi_{d}\right\}$ da seguinte maneira

$$
M(\Psi(f(\xi)))=\sum_{k=1}^{d}\left(M(\Psi(f(\xi))), \varphi_{k}\right)_{H} \varphi_{k}
$$

O que em conjunto com (8.2.23) nos dá

$$
\begin{aligned}
\left\|\sum_{k=1}^{d} \frac{\partial \Gamma}{\partial \xi_{k}}(\xi) \varphi_{k}-M(\Psi(f(\xi)))\right\|_{V^{\prime}} & =\left\|\sum_{k=1}^{d}\left[\left(M(\xi(f(\xi))), \Psi^{\prime}(f(\xi)) \varphi_{k}-\varphi_{k}\right)_{H}\right] \varphi_{k}\right\|_{V^{\prime}} \\
& \leq C_{3}\|M(\Psi(f(\xi)))\|_{V^{\prime}} \sum_{k=1}^{d}\left\|\Psi^{\prime}(f(\xi)) \varphi_{k}-\varphi_{k}\right\|_{V}
\end{aligned}
$$

para alguma constante $C_{3}>0$ dependendo somente da imersão $V \hookrightarrow H$ e da escolha dos vetores da base $\left\{\varphi_{1}, \ldots, \varphi_{d}\right\}$ de $\operatorname{ker}\left(L^{n_{0}}\right)$.

Por outro lado, como $\Psi^{\prime}(0)=\mathcal{L}^{-1}$ e $\mathcal{L} \varphi_{k}=\Pi \varphi_{k}+L \varphi_{k}=\varphi_{k}$, a desigualdade acima fica

$\left\|\sum_{k=1}^{d} \frac{\partial \Gamma}{\partial \xi_{k}}(\xi) \varphi_{k}-M(\Psi(f(\xi)))\right\|_{V^{\prime}} \leq C_{3}\|M(\Psi(f(\xi)))\|_{V^{\prime}}\left\|\Psi^{\prime}(f(\xi))-\Psi^{\prime}(0)\right\|_{\mathcal{L}\left(V^{\prime}, V\right)} \sum_{k=1}^{d}\left\|\varphi_{k}\right\|_{V}$.

Da continuidade da aplicação derivada $\Psi^{\prime}: V^{\prime} \rightarrow \mathcal{L}\left(V^{\prime}, V\right)$, deduz-se a existência de uma função contínua $\rho: \widetilde{W}_{2}(0) \rightarrow[0, \infty)$ com $\rho(0)=0$ tal que

$$
\left\|\sum_{k=1}^{d} \frac{\partial \Gamma}{\partial \xi_{k}}(\xi) \varphi_{k}-M(\Psi(f(\xi)))\right\|_{V^{\prime}} \leq C_{3} \rho(\xi)\|M(\Psi(f(\xi)))\|_{V^{\prime}}
$$

Portanto, diminuindo o conjunto aberto $\widetilde{W}_{2}(0)$, se necessário, podemos assumir que

$$
\|M(\Psi(f(\xi)))\|_{V^{\prime}} \leq C\|\nabla \Gamma(\xi)\|
$$

para todo $\xi \in \widetilde{W}_{2}(0)$ e uma certa constante $C>0$.

Por outro lado, da continuidade de $\Psi^{\prime}$ e usando o fato de que $\operatorname{ker}\left(L^{n_{0}}\right)$ possui dimensão finita, (8.2.24) também nos dá

$$
\|\nabla \Gamma(\xi)\| \leq C_{4}\|M(\Psi(f(\xi)))\|_{V^{\prime}}
$$

para uma certa constante $C_{4}>0$. Combinando isto e repetindo manipulação análoga a que fizemos acima obtém-se

$$
\begin{aligned}
\|\nabla \Gamma(\xi)\| & \leq C_{4}\|M(\Psi(f(\xi)))\|_{V^{\prime}} \leq C_{4}\|M(\Psi(f(\xi)))-M(u)\|_{V^{\prime}}+C_{4}\|M(u)\|_{V^{\prime}} \\
& \leq C_{5}\|\Psi(f(\xi))-u\|_{V}+C_{4}\|M(u)\|_{V^{\prime}} \leq C_{6}\|M(u)\|_{V^{\prime}}
\end{aligned}
$$


onde usamos (8.2.19).

Resumindo, obtivemos

$\|\nabla \Gamma(\xi)\| \leq C_{6}\|M(u)\|_{V^{\prime}}$, para todo $u \in \tilde{W}_{1}(0)$ e $\xi \in \widetilde{W}_{2}(0) \operatorname{com} f(\xi)=\Pi u$. (8.2.26)

Agora, afirmamos que se verifica a seguinte igualdade:

$$
\left\{u \in \widetilde{W}_{1}(0): M(u)=0\right\}=\Psi\left(\left\{f(\xi): \xi \in \widetilde{W}_{2}(0) \text { e } \nabla \Gamma(\xi)=0\right\}\right) .
$$

De fato, consideremos $u_{0} \in \widetilde{W}_{1}(0)$ com $M\left(u_{0}\right)=0$. Então, $u_{0}=\Psi\left(\mathcal{N}\left(u_{0}\right)\right)=$ $\Psi\left(\Pi u_{0}\right)$, e se $\xi_{0} \in \widetilde{W}_{2}(0)$ é tal que $f\left(\xi_{0}\right)=\Pi u_{0}$, conclui-se que $u_{0}=\Psi\left(f\left(\xi_{0}\right)\right)$. Levando em conta (8.2.26) vemos que $\nabla \Gamma\left(\xi_{0}\right)=0$, e por isto a inclusão

$$
\left\{u \in \widetilde{W}_{1}(0): M(u)=0\right\} \subset \Psi\left(\left\{f(\xi): \xi \in \widetilde{W}_{2}(0) \text { e } \nabla \Gamma(\xi)=0\right\}\right)
$$

é válida.

Para estabelecer a inclusão contrária, seja $u_{0}=\Psi\left(f\left(\xi_{0}\right)\right) \in W_{1}(0) \operatorname{com} \xi_{0} \in \widetilde{W}_{2}(0)$ e $\nabla \Gamma\left(\xi_{0}\right)=0$. Segue-se de (8.2.25) que $M\left(u_{0}\right)=0$, portanto

$$
\Pi u_{0}=\Pi \Psi\left(f\left(\xi_{0}\right)\right)=\Pi \Psi\left(f\left(\xi_{0}\right)\right)+M\left(\Psi\left(f\left(\xi_{0}\right)\right)\right)=\mathcal{N}\left(\Psi\left(f\left(\xi_{0}\right)\right)\right)=f\left(\xi_{0}\right) \in W_{2}(0),
$$

e $\operatorname{assim} u_{0} \in \widetilde{W}_{1}(0)$, como queríamos.

Finalmente, afirmamos que $\Gamma(\xi)=0$ para todo $\xi$ suficientemente pequeno.

Com efeito, levando em conta a hipótese $(i i)$, dado que $\left\{u \in \widetilde{W}_{1}(0): M(u)=0\right\}=$ $\widetilde{W}_{1}(0) \cap M^{-1}(0)$ é um aberto de $M^{-1}(0)$ com a topologia induzida de $V$, segue-se que $\widetilde{U}:=h^{-1}\left(\left\{u \in \widetilde{W}_{1}(0): M(u)=0\right\}\right)$ é um aberto de $\mathbb{R}^{d} \mathrm{e}$

$$
h(\widetilde{U})=\left\{u \in \widetilde{W}_{1}(0): M(u)=0\right\} \cap h(U) \subset \Psi\left(\left\{f(\xi): \xi \in \widetilde{W}_{2}(0)\right\}\right)=(\Psi \circ f)\left(\widetilde{W}_{2}(0)\right) .
$$

Observemos que $h(\widetilde{U})$ e $(\Psi \circ f)\left(\widetilde{W}_{2}(0)\right)$ são variedades topológicas com mesma dimensão $d$, portanto, $h(\widetilde{U})$ deve ser um aberto de $(\Psi \circ f)\left(\widetilde{W}_{2}(0)\right)$ (confira "Brouwer Domain Invariance Theorem" em [23]). Assim, diminuindo o aberto $\widetilde{W}_{2}(0)$, se necessário, podemos supor a seguinte igualdade

$$
\left\{u \in \widetilde{W}_{1}(0): M(u)=0\right\}=h(\widetilde{U})=(\Psi \circ f)\left(\widetilde{W}_{2}(0)\right) .
$$

Isto em conjunto com (8.2.27) nos dá

$$
\Psi\left(\left\{f(\xi): \xi \in \widetilde{W}_{2}(0) \text { e } \nabla \Gamma(\xi)=0\right\}\right)=\Psi\left(\left\{f(\xi): \xi \in \widetilde{W}_{2}(0)\right\}\right),
$$

por isso, $\nabla \Gamma(\xi)=0$ qualquer que seja $\xi \in \widetilde{W}_{2}(0)$.

Sem perda de generalidade, podemos supor, além disso, que $\widetilde{W}_{2}(0)$ é conexo, donde deduz-se que $\Gamma$ é constante em $\widetilde{W}_{2}(0)$ e, uma vez que $\Gamma(0)=0$, resulta que $\Gamma(\xi)=0$ qualquer que seja $\xi \in \widetilde{W}_{2}(0)$, o que conclui a prova do teorema. 
Observação 8.2.2. Suponhamos que $\operatorname{ker}(L)$ na hipótese $(i i)$ acima seja não trivial. Resulta, mantendo a mesma notação que na prova do teorema, que a seguinte igualdade se verifica

$$
V=N \oplus\left(V \cap R\left(I_{V^{\prime}}-\Pi\right)\right)=: N \oplus N_{0}
$$

Observemos que $\left.L\right|_{N_{0}}: N_{0} \rightarrow V^{\prime}$, que é exatamente a derivada parcial de $M$ no ponto $\varphi$ ao longo de $N_{0}$, é injetiva e, se fosse também sobrejetiva, o mesmo ocorreria com o operador original $L: V \rightarrow V^{\prime}$, donde, pela Alternativa de Fredholm, levando em conta $(i i), \Lambda^{-1} L=I_{V}+\Lambda^{-1} B$ seria um isomorfismo e portanto $L: V \rightarrow V^{\prime}$ deveria ser isomorfismo também. Ou seja, por meio deste argumento, não podemos obter a hipótese (iii), utilizando o Teorema da função implícita.

Agora, para terminar o capítulo, apresentamos um exemplo bastante simples onde podemos verificar facilmente as hipóteses suficientes para a desiguladade de $\mathcal{L}$ ojasiewiczSimon.

Exemplo 8.2.3. Seja $g: \mathbb{R} \rightarrow \mathbb{R}$ um função $C^{2}(\mathbb{R})$ tal que $g(s) \neq 0$ para todo $s \in \mathbb{R}$ e consideremos a função $G: \mathbb{R}^{2} \rightarrow \mathbb{R}$ definida por $G(s, t):=t^{2} g(s)$.

Segue imediatamente que $G \in C^{2}\left(\mathbb{R}^{2}, \mathbb{R}\right) \operatorname{com} M(s, t):=G^{\prime}(s, t)=\left(t^{2} g^{\prime}(s), 2 t g(s)\right)$ e

$$
G^{\prime \prime}(s, t)=\left(\begin{array}{cc}
t^{2} g^{\prime \prime}(s) & 2 t g^{\prime}(s) \\
2 t g^{\prime}(s) & 2 g(s)
\end{array}\right)
$$

para todo $(s, t) \in \mathbb{R}^{2}$.

Em particular, $G^{\prime \prime}(s, t)$ é uma matriz simétrica para todos os reais $s$ e $t$, e assim a projeção ortogonal coincide com a espectral.

É claro que $M^{-1}(0)=\mathbb{R} \times\{0\}$ e portanto, uma vez que $G^{\prime \prime}(s, 0)=\left(\begin{array}{cc}0 & 0 \\ 0 & 2 g(s)\end{array}\right)$, resulta que $\operatorname{dim} \operatorname{ker}\left(G^{\prime \prime}\left(x^{*}\right)\right)=1$ para todo $x^{*} \in M^{-1}(0)$. Agora, a aplicação $h: \mathbb{R} \rightarrow$ $\mathbb{R} \times \mathbb{R}$ dada por $h(s):=(s, 0)$ é um homeomorfismo entre $\mathbb{R}$ e $M^{-1}(0)$. Assim, a aplicação $G$ satisfaz a hipótese (ii) do Teorema 8.2.1.

Por outro lado, seja $Y$ um espaço de Banach e $f: \mathbb{R}^{2} \times Y \rightarrow Y$ uma aplicação suave tal que para todo subconjunto limitado $B \subset \mathbb{R}^{2}$ sua restrição a $B \times Y$ é limitada.

Seja também $-A: D(A) \subset Y \rightarrow Y$ um operador setorial em $Y$ tal que o semigrupo analítico gerado por seu oposto, $\left\{e^{A t}: t \geq 0\right\}$, satisfaça o decaimento exponencial $\left\|e^{A t}\right\|_{\mathcal{L}(Y)} \leq M e^{-\delta t}$ para todo real $t>0$ e certas constantes $M \geq 1$ e $\delta>0$.

Nestas condições, usando os resultados de [7] sobre existência de atratores pullback, não é difícil ver que, para todo solução limitada $\varphi: \mathbb{R} \rightarrow \mathbb{R}^{2}$ da equação $x^{\prime}+M(x)=0$, a aplicação $f_{\varphi}: \mathbb{R} \times Y \rightarrow Y$, dada por $f_{\varphi}(t, y):=f(\varphi(t), y)$, é compatível à direita e à 
esquerda com respeito ao sistema

$$
\left\{\begin{array}{l}
y^{\prime}=A y+f_{\varphi}(t, y) \quad t>\tau \\
y(\tau)=y_{0} \in Y
\end{array}\right.
$$

Se para todo ponto de equilíbrio $x^{*} \in M^{-1}(0)$, os equilíbrios do problema $y^{\prime}=A y+$ $f\left(x^{*}, y\right) t>0$ forem todos hiperbólicos, então

$$
\left\{\begin{array}{l}
x^{\prime}+M(x)=0 \quad t>0 \\
y^{\prime}=A y+f(x, y) \quad t>0 \\
x(0)=x_{0} \in \mathbb{R}^{2}, \quad y(0)=y_{0} \in Y
\end{array}\right.
$$

é de tipo gradiente.

\subsection{Aplicação da desigualdade de Lojasiewicz-Simon a problemas abstratos}

Nesta seção aplicamos o Teorema 8.2.1 no estudo de alguns problemas abstratos, no contexto dos espaços de Hilbert, tanto no caso de equações de primeira ordem (como em esquações tipo a do calor) como de segunda (equações tipo da onda).

Os resultados que apresentamos aqui são, de certa forma, novos, mas estando fortemente inspirados no trabalho [24], onde se estudou a convergência de soluções para pontos estacionários, no caso de um conjunto infinito de equilíbrios, e fazendo-se $t \rightarrow \infty$. Nós, por outro lado, trataremos aqui o caso $t \rightarrow-\infty$ e registremos que, ainda que não apresentamos, em detalhes, o caso $t \rightarrow \infty$, a prova não é inteiramente análoga.

\subsubsection{Equações de primeira ordem}

O lema que apresentamos a seguir desempenha um papel importante nos resultados desta e da próxima subseção. Ele nos proporciona estimativas exponenciais para um caminho $u$ conhecendo-se estimativas exponenciais para a norma $L^{2}$ de sua derivada. Mais precisamente, temos:

Lema 8.3.1. Seja $u:(-\infty, 0] \rightarrow H$ um caminho diferenciável para o qual existem constantes $a>0$ e $\gamma>0$ satisfazendo, para algum $T>0$ e todo $t \in[-T, 0]$

$$
\int_{-\infty}^{t}\|\dot{u}(s)\|_{H}^{2} d s \leq a e^{\gamma t}
$$

Então, para todos os reais $\tau$ e $t$ em $[-T, 0]$, com $\tau \leq t$, temos

$$
\|u(t)-u(\tau)\|_{H} \leq b \sqrt{a} e^{\frac{\gamma}{2} t},
$$

onde $b:=\frac{e^{\frac{\gamma}{2}}}{e^{\frac{\gamma}{2}}-1}$. 
Demonstração: Com efeito, dados $\tau \leq t \leq 0$, suponhamos, primeiramente, que $t-\tau \leq 1$, então, pela desigualdade de Hölder e por (8.3.28), temos

$$
\begin{aligned}
\|u(t)-u(\tau)\|_{H} & =\left\|\int_{\tau}^{t} \dot{u}(s) d s\right\|_{H} \leq \int_{\tau}^{t}\|\dot{u}(s)\|_{H} d s \\
& \leq \sqrt{t-\tau}\left(\int_{\tau}^{t}\|\dot{u}(s)\|_{H}^{2} d s\right)^{\frac{1}{2}} \leq \sqrt{a} e^{\frac{\gamma}{2} t} .
\end{aligned}
$$

Agora, se $t-\tau>1$ seja $n$ o menor inteiro positivo tal que $(t-\tau)-n \leq 1$. Então, segue-se do fizemos acima que

$$
\begin{gathered}
\|u(t)-u(\tau)\|_{H} \leq\|u(t-n)-u(\tau)\|_{H}+\sum_{j=0}^{n-1}\|u(t-j)-u(t-j-1)\|_{H} \leq \\
\sqrt{a} e^{\frac{\gamma}{2}(t-n)}+\sum_{j=0}^{n-1} \sqrt{a} e^{\frac{\gamma}{2}(t-j)}=\sqrt{a} e^{\frac{\gamma}{2} t}\left[e^{-\frac{\gamma}{2} n}+\sum_{j=0}^{n-1} e^{-\frac{\gamma}{2} j}\right] \leq \sqrt{a} e^{\frac{\gamma}{2} t}\left[\frac{e^{\frac{\gamma}{2}}}{e^{\frac{\gamma}{2}}-1}\right],
\end{gathered}
$$

e o lema está demonstrado.

A seguir, enunciamos o análogo do lema anterior para o caso de caminhos diferenciáveis definidos no intervalo $[0, \infty)$, sua demonstração, por ser semelhante à anterior, será omitida (confira [24], Lemma 2.2).

Lema 8.3.2. Seja $u:[0, \infty) \rightarrow H$ um caminho diferenciável tal que, para um certo par de constantes $a>0$ e $\gamma>0$, para algum $T>0$ e todo $t \in[0, T]$

$$
\int_{t}^{\infty}\|\dot{u}(s)\|_{H}^{2} d s \leq a e^{-\gamma t}
$$

Então,

$$
\|u(t)-u(\tau)\|_{H} \leq b \sqrt{a} e^{-\frac{\gamma}{2} t}
$$

toda vez que $\tau$ e $t$ são reais em $[0, T]$, com $t \leq \tau$, sendo $b:=\frac{e^{\frac{\gamma}{2}}}{e^{\frac{\gamma}{2}}-1}$.

De posse destes dois últimos resultados podemos provar o

Teorema 8.3.3. Suponhamos que as hipóteses do Teorema 8.2.1 estejam satisfeits para toda $\varphi \in \mathcal{S}:=\{x \in V: M(x)=0\}$ e seja $u: \mathbb{R} \rightarrow V$ uma solução global do problema

$$
\left\{\begin{array}{l}
\dot{u}+M(u)=0, t>0 \\
u(0)=u_{0} \in V
\end{array},\right.
$$

(o qual se supõe gerar semigrupo não lienar no sentido que explicamos antes) tal que a órbita $\gamma_{u}\left(u_{0}\right):=\{u(t) \in V: t \in \mathbb{R}\}$ é relativamente compacta em $V$. Então, existem $\varphi$ e $\psi$ em $\mathcal{S}$ tais que

$$
\lim _{t \rightarrow-\infty}\|u(t)-\varphi\|_{V}=0 e \lim _{t \rightarrow \infty}\|u(t)-\psi\|_{V}=0 .
$$


Demonstração: Estudamos somente o caso $t \rightarrow-\infty$, uma vez que o caso $t \rightarrow \infty$ se encontra feito em [24], Theorem 1.1.

Observemos, inicialmente, que se $\xi: \mathbb{R} \rightarrow V$ é uma solução global qualquer para (8.3.29), então para todo $t \in \mathbb{R}$ tem-se

$$
\frac{d}{d t}(G \circ \xi)(t)=\langle M(\xi(t)), \dot{\xi}(t)\rangle=-\|\dot{\xi}(t)\|_{H}^{2}=-\|M(\xi(t))\|_{H}^{2} \leq 0 .
$$

Donde, a função real $\mathbb{R} \ni t \mapsto G(\xi(t)) \in \mathbb{R}$ é não crescente. Este fato, juntamente com a igualdade $\frac{d}{d t}(G \circ \xi)(t)=-\|\dot{\xi}(t)\|_{H}^{2}$, dizem que $G: V \rightarrow \mathbb{R}$ é uma função de Lyapunov para o semigrupo em $V$ associado ao sistema (8.3.29). Portanto, o conjunto $\alpha$-limite de $u_{0}$ segundo a solução $u, \alpha_{u}\left(u_{0}\right)=\left\{\varphi \in V\right.$ : existe $\left(t_{n}\right)_{n \in \mathbb{N}}$ em $\mathbb{R}$ com $t_{n} \rightarrow-\infty$ tal que $\left.\lim _{n \rightarrow \infty}\left\|u\left(t_{n}\right)-\varphi\right\|_{V}=0\right\}$, está contido em $\mathcal{S}$ e é não vazio pela hipótese de pré-compacidade de $\gamma_{u}\left(u_{0}\right)=\{u(t) \in V: t \in \mathbb{R}\}$.

Dada $\varphi \in \alpha_{u}\left(u_{0}\right)$, existe $\left(t_{n}\right)_{n \in \mathbb{N}}$ em $\mathbb{R}$ com $t_{n} \rightarrow-\infty$ tal que

$$
\lim _{n \rightarrow \infty}\left\|u\left(t_{n}\right)-\varphi\right\|_{V}=0
$$

$\log 0 \lim _{n \rightarrow \infty} G\left(u\left(t_{n}\right)\right)=G(\varphi)$ e pela monotonicidade da função $\mathbb{R} \ni t \mapsto G(u(t)) \in \mathbb{R}$ temos $\lim _{t \rightarrow-\infty} G(u(t))=G(\varphi)$ com $G(u(t)) \leq G(\varphi)$ qualquer que seja $t \in \mathbb{R}$.

Por um lado, para todo $t$ real temos

$$
\frac{d}{d t}[(G \circ u)(t)-G(\varphi)]=\langle M(u(t)), \dot{u}(t)\rangle=-\|\dot{u}(t)\|_{H}^{2}=-\|M(u(t))\|_{H}^{2} .
$$

Então, fixado $t<0$ e integrando a igualdade acima de $-\infty$ até $t$ resulta que

$$
\int_{-\infty}^{t}\|\dot{u}(s)\|_{H}^{2} d s=[G(\varphi)-G(u(t))]
$$

Por outro, para cada natural $j$ escolhamos $n_{j} \in \mathbb{N}$ de maneira que $n_{1}<n_{2}<\cdots<$ $n_{j}<\cdots \mathrm{e}$

$$
\left\|u\left(t_{n_{j}}\right)-\varphi\right\|_{V}<\frac{1}{j} \text { e }\left[G(\varphi)-G\left(u\left(t_{n_{j}}\right)\right)\right]^{\frac{1}{2}}<\frac{1}{j} .
$$

Agora, seja $\sigma>0$ como no Teorema 8.2.1 associado ao equilíbrio $\varphi$ e $j_{0}$ tal que $\frac{1}{j}<\sigma$ sempre que $j \geq j_{0}$. Definamos então, para $j \geq j_{0}$

$$
\bar{t}_{j}:=\inf \left\{\tau<t_{n_{j}}:\|u(t)-\varphi\|_{V}<\sigma \text { para todo } t \in\left[\tau, t_{n_{j}}\right]\right\} .
$$

Segue-se da Desigualdade de Lojasiewicz-Simon e da inclusão contínua de $H$ em $V^{\prime}$ que existe uma constante $\gamma>0$ dependendo somente de $\varphi$ tal que que para $t \in\left[\bar{t}_{j}, t_{n_{j}}\right]$ de (8.3.30) temos

$$
\frac{d}{d t}[(G \circ u)(t)-G(\varphi)] \leq-\gamma[G(\varphi)-G(u(t))]=\gamma[G(u(t))-G(\varphi)]
$$


donde resulta que

$$
\begin{aligned}
\frac{d}{d t}[(G \circ u)(t)-G(\varphi)]-\gamma[G(u(t))-G(\varphi)] & \leq 0 \Rightarrow \\
e^{-\gamma t} \frac{d}{d t}[(G \circ u)(t)-G(\varphi)]-\gamma e^{-\gamma t}[G(u(t))-G(\varphi)] & \leq 0 \Rightarrow \\
\frac{d}{d t}\left\{e^{-\gamma t}[G(u(t))-G(\varphi)]\right\} & \leq 0
\end{aligned}
$$

e integrando esta última desigualdade de $t \in\left(\bar{t}_{j}, t_{n_{j}}\right)$ até $t_{n_{j}}$ obtém-se que

$$
\begin{gathered}
e^{-\gamma t_{n_{j}}}\left[G\left(u\left(t_{n_{j}}\right)\right)-G(\varphi)\right]-e^{-\gamma t}[G(u(t))-G(\varphi)] \leq 0 \Rightarrow \\
e^{-\gamma t}[G(\varphi)-(G \circ u)(t)] \leq e^{-\gamma t_{n_{j}}}\left[G(\varphi)-(G \circ u)\left(t_{n_{j}}\right)\right] \Rightarrow \\
{[G(\varphi)-(G \circ u)(t)] \leq e^{-\gamma\left(t_{n_{j}}-t\right)}\left[G(\varphi)-(G \circ u)\left(t_{n_{j}}\right)\right] \Rightarrow} \\
{[G(\varphi)-(G \circ u)(t)] \leq e^{\gamma t}\left\{e^{-\gamma t_{n_{j}}}\left[G(\varphi)-(G \circ u)\left(t_{n_{j}}\right)\right]\right\} .}
\end{gathered}
$$

Levando em conta esta última desigualdade em (8.3.31) conclui-se que

$$
\int_{-\infty}^{t}\|\dot{u}(s)\|_{H}^{2} d s \leq e^{\gamma t}\left\{e^{-\gamma t_{n_{j}}}\left[G(\varphi)-(G \circ u)\left(t_{n_{j}}\right)\right]\right\}
$$

para todo $t \in\left(\bar{t}_{j}, t_{n_{j}}\right)$ sempre que $j \geq j_{0}$.

Nestas condições, afirmamos que existe $j_{1} \geq j_{0}$ tal que $\bar{t}_{j_{1}}=-\infty$.

Com efeito, no caso em que $G(\varphi)=(G \circ u)\left(t_{n_{j}}\right)$ para algum $j$, a conclusão é óbvia (porque obteremos que $u(t)=\varphi$ qualquer que seja $t \geq t_{n_{j}}$, pelo fato de que $G: V \rightarrow \mathbb{R}$ é função de Lyapunov para o semigrupo gerado por (8.3.29)).

Caso contrário, suponhamos que para todo $j \geq j_{0}$ se tenha $\bar{t}_{j}>-\infty$ e então de (8.3.32) podemos aplicar o Lema $8.3 .1 \operatorname{com} \tau:=\bar{t}_{j}, t:=t_{n_{j}}$ e $a:=e^{-\gamma t_{n_{j}}}[G(\varphi)-(G \circ$ $\left.u)\left(t_{n_{j}}\right)\right]$ para obtermos

$$
\left\|u\left(\bar{t}_{j}\right)-u\left(t_{n_{j}}\right)\right\|_{H}<b e^{\frac{\gamma}{2}\left(\bar{t}_{j}-t_{n_{j}}\right)} \sqrt{\left[G(\varphi)-(G \circ u)\left(t_{n_{j}}\right)\right]}<\frac{b}{j},
$$

qualquer que seja $j \geq j_{0}$, onde $b>0$ é uma constante que depende somente de $\gamma$.

Além disso, observemos que $\left\|u\left(t_{n_{j}}\right)-\varphi\right\|_{H}<\frac{C_{0}}{j}$ para todo $j \geq j_{0}$, onde $C_{0}$ é a constante que vem da continuidade da inclusão $V \hookrightarrow H$.

Resumindo, obtivemos que para todo $j \geq j_{0}$

$$
\left\|u\left(\bar{t}_{j}\right)-\varphi\right\|_{H} \leq\left\|u\left(\bar{t}_{j}\right)-u\left(t_{n_{j}}\right)\right\|_{H}+\left\|u\left(t_{n_{j}}\right)-\varphi\right\|_{H}<\frac{b}{j}+\frac{C_{0}}{j},
$$


mostrando, evidentemente, que $\lim _{j \rightarrow \infty}\left\|u\left(\bar{t}_{j}\right)-\varphi\right\|_{H}=0$. Mas, pela pré-compacidade de $\gamma_{u}\left(u_{0}\right)$ em $V$ podemos supor que a sequência $\left(u\left(\bar{t}_{j}\right)\right)_{j \in \mathbb{N}}$ converge em $V$ para $\varphi$, donde existe $j^{*}$ satisfazendo

$$
\left\|u\left(\bar{t}_{j^{*}}\right)-\varphi\right\|_{V}<\sigma
$$

contradizendo a definição de $\bar{t}_{j^{*}}$, consequentemente deve existir $j_{1} \geq j_{0}$ tal que $\bar{t}_{j}=-\infty$ qualquer que seja $j \geq j_{1}$.

Assim, podemos repetir a construção realizada acima (a mesma que resultou em (8.3.32)) para obter, qualquer que seja $j \geq j_{1}$, que

$$
\int_{-\infty}^{t}\|\dot{u}(s)\|_{H}^{2} d s \leq e^{\gamma t}\left\{e^{-\gamma t_{n_{j}}}\left[G(\varphi)-(G \circ u)\left(t_{n_{j}}\right)\right]\right\} \text { sempre que } t \in\left(-\infty, t_{n_{j}}\right)
$$

e, mais uma vez, por aplicação do Lema 8.3.1, obter, toda vez que $j \geq j_{1}$ e $t \in$ $\left(-\infty, t_{n_{j}}\right)$, o seguinte

$$
\left\|u(t)-u\left(t_{n_{j}}\right)\right\|_{H}<b e^{\frac{\gamma}{2}\left(t-t_{n_{j}}\right)} \sqrt{\left[G(\varphi)-(G \circ u)\left(t_{n_{j}}\right)\right]}<\frac{b}{j},
$$

o que implica em

$$
\|u(t)-\varphi\|_{H} \leq\left\|u(t)-u\left(t_{n_{j}}\right)\right\|_{H}+\left\|u\left(t_{n_{j}}\right)-\varphi\right\|_{H}<\frac{b}{j}+\frac{C_{0}}{j}, \text { para } t \in\left(-\infty, t_{n_{j}}\right)
$$

provando que $\lim _{t \rightarrow-\infty}\|u(t)-\varphi\|_{H}=0$ e, novamente, utilizando-se a pré-compacidade de $\gamma_{u}\left(u_{0}\right)$ em $V$ e recordando-se que, em um espaço métrico, se uma sequência for tal que todas as suas subsequências possuem uma subsequência convergindo para um mesmo limite, então a sequência original converge para este limite comum, conclui-se que $\lim _{t \rightarrow-\infty}\|u(t)-\varphi\|_{V}=0$, completando a demonstração do teorema.

É importante levarmos em conta que a Proposição 2.1 em [25] não contradiz o teorema anterior. Para tornarmos mais clara esta afirmação, enunciemos e demonstremos tal proposição aqui e a seguir justificamos.

Proposição 8.3.4. Seja $R: \mathbb{R} \rightarrow \mathbb{R}$ uma função de classe $C^{\infty}$ positiva e $2 \pi$-periódica e definamos, para $\varepsilon \geq 0$, os seguintes conjuntos:

$$
S:=\left\{(\rho \cos \gamma, \rho \sin \gamma): \rho=\frac{1}{R(\gamma)}, \gamma \in[0,2 \pi)\right\}
$$

e

$$
B_{\varepsilon}:=\left\{(\rho \cos \gamma, \rho \sin \gamma): 0 \leq \rho<\frac{1+\varepsilon}{R(\gamma)}, \gamma \in[0,2 \pi)\right\} .
$$

Observemos que a fronteira de $B_{0}$ é igual a $S$. Em símbolos $\partial B_{0}=S$. 
Seja também $G: \mathbb{R}^{2} \rightarrow \mathbb{R}$ uma função de classe $C^{2}$ satisfazendo as seguintes propriedades:

$(P 1) G(u)=0$ qualquer que seja $u \in \overline{B_{0}}$ (portanto $M(u):=\nabla G(u)=0$ para todo $\left.u \in \overline{B_{0}}\right)$.

$(P 2)$ Existem funções de classe $C^{1} l_{1}, l_{2}:(0, \infty) \rightarrow \mathbb{R}$ tais que

$$
\begin{gathered}
\frac{1}{R(\gamma)}<l_{2}(\gamma+2 \pi)<l_{1}(\gamma)<l_{2}(\gamma), \gamma>0 . \\
l_{2}(\gamma)-\frac{1}{R(\gamma)} \rightarrow 0 \text { quando } \gamma \rightarrow \infty
\end{gathered}
$$

e existe $\varepsilon>0$ tal que

$$
Z:=\left\{u \in B_{\varepsilon}: G(u)=0\right\}=\overline{B_{0}} \cup\left(L_{1} \cap B_{\varepsilon}\right) \cup\left(L_{2} \cap B_{\varepsilon}\right),
$$

onde

$$
L_{i}:=\left\{(\rho \cos \gamma, \rho \sin \gamma): \rho=l_{i}(\gamma), \gamma>0\right\}, i=1,2
$$

(P3) Para o $\varepsilon>0$ em (P2) tem-se $M(u)=\nabla G(u) \neq 0$ para todo $u \in B_{\varepsilon} \backslash \overline{B_{0}}$.

Finalmente, seja $\Theta: \mathbb{R}^{2} \rightarrow M_{2}(\mathbb{R})$ uma aplicação de classe $C^{1}$, de duas variáveis reais a valores matriciais, que faz corresponder a cada $u \in \mathbb{R}^{2}$ uma matriz $\Theta(u)$ simétrica e positiva definida e consideremos o sistema gradiente no plano:

$$
\left\{\begin{array}{c}
\dot{u}=-\Theta(u) \nabla G(u) \\
u(0)=u_{0} \in \mathbb{R}^{2}
\end{array} .\right.
$$

Nestas condições, existe um dado inicial $u_{0} \in B_{\varepsilon}$ tal que a solução de (8.3.33) passando por $u_{0}$ existe para todo $t \geq 0$ é limitada e seu conjunto $\omega$-limite é igual ao conjunto $S$ e, portanto, um contínuo de equilíbrios homeomorfo a $S^{1}:=\{z \in \mathbb{C}:|z|=$ $1\}$.

Demonstração: Com efeito, por $(P 1)$ e $(P 3)$ temos que o conjunto de pontos de equilíbrio de (8.3.33) em $B_{\varepsilon}$ coincide com $\overline{B_{0}}$. Agora, observemos que a função $G: \mathbb{R}^{2} \rightarrow \mathbb{R}$ é uma função de Lyapunov para (8.3.33), uma vez que, se $\xi: J \rightarrow \mathbb{R}^{2}$ é uma solução qualquer do sistema, definida em um intervalo $J$, então

$$
\frac{d}{d t}(G \circ \xi)(t)=-(\nabla G(\xi(t)), \Theta(\xi(t)) \nabla G(\xi(t))) \leq 0
$$

(onde $(\cdot, \cdot)$ indica o produto escalar euclidiano usual de $\left.\mathbb{R}^{2}\right)$ pois $\Theta(\xi(t))$ é positiva definida para todo $t$. Desta forma, toda solução de (8.3.33) definida e limitada para todo $t \geq 0$ deve ter conjunto $\omega$-limite não vazio e formado somente por pontos de equilíbrio do sistema. 
Observemos que, pela continuidade da função real $F: \mathbb{R}^{2} \times \mathbb{R}^{2} \rightarrow \mathbb{R}, F(\xi, \zeta)=$ $(\zeta, \Theta(\xi) \zeta)$ e pela positividade dos $\Theta(\xi)$ 's, segue-se que para todo compacto $K \subset \mathbb{R}^{2}$ existe uma constante $c(K)>0$ de modo que

$$
(\zeta, \Theta(\xi) \zeta) \geq c(K)|\zeta|^{2}, \text { quaisquer que sejam } \xi \in K \text { e } \zeta \in \mathbb{R}^{2}
$$

Agora, definamos o conjunto aberto

$$
H:=\left\{(\rho \cos \gamma, \rho \sin \gamma): l_{1}(\gamma)<\rho<l_{2}(\gamma), \gamma \geq N\right\}
$$

onde $N>0$ é escolhido de forma que se tenha $H \subset B_{\varepsilon}$. Substituindo $G$ por $-G$ na equação, se necessário, podemos, por $(P 2)$ e a conexidade por caminhos de $H$, supor que $G(u)>0$ para todo $u \in H$.

Afirmamos que existem pontos $P_{1}$ e $P_{2}$ em $H$ com a propriedade de que $\xi_{i}$, a solução de (8.3.33) por $P_{i}$, deixa o conjunto $H$ passando por $L_{i} \cap B_{\varepsilon}$ para $i=1,2$. Com efeito, para fixar ideias, escolhamos um ponto $z_{0} \in L_{1} \cap B_{\varepsilon}$ e seja $\xi_{0}:\left(\omega_{-}, \omega_{+}\right) \rightarrow \mathbb{R}^{2}$ a solução maximal de (8.3.33) $\operatorname{com} \xi_{0}(0)=z_{0}$. Seja $J:=[a, b]$ um intervalo compacto contido em $\left(\omega_{-}, \omega_{+}\right)$tal que $0 \in(a, b)$ e $\xi_{0}(t) \in B_{\varepsilon} \backslash \overline{B_{0}}$ para todo $t \in J$. Pondo $2 \mu:=\operatorname{dist}\left(K_{0}, \partial\left(B_{\varepsilon} \backslash \overline{B_{0}}\right)\right)>0$, onde $K_{0}:=\left\{\xi_{0}(t): t \in J\right\}$, fixemos $c(K)>0$ como em (8.3.35) acima, correspondendo ao conjunto compacto $K:=\overline{\mathcal{O}_{\mu}\left(K_{0}\right)}=\left\{\xi \in \mathbb{R}^{2}\right.$ : $\left.\operatorname{dist}\left(\xi, K_{0}\right) \leq \mu\right\}$, e fixemos também o número positivo $m_{K}:=\inf _{\xi \in K}|\nabla G(\xi)|^{2}>0$. Escolhamos, arbitrariamente, uma sequência $\left(z_{n}\right)_{n \in \mathbb{N}}$ em $H$ com $z_{n} \rightarrow z_{0}$, portanto $G\left(z_{n}\right) \rightarrow G\left(z_{0}\right)=0$. Fixemos, além disso, $n_{1} \in \mathbb{N}$ tal que

$$
\tau_{n}:=\frac{G\left(z_{n}\right)}{c(K) m_{K}} \in(0, b), \text { para todo } n \geq n_{1} .
$$

Finalmente, sejam

$$
p:=\min \left\{\mu, \operatorname{dist}\left(K, L_{2}\right)\right\}>0
$$

e $\eta>0 \mathrm{com}$

$$
0<\eta<p .
$$

Pela continuidade com relação aos dados iniciais, ao $\eta>0$ acima e ao intervalo $\left[0, \tau_{n_{1}}\right]$ corresponde $\delta>0$ tal que se $z$ é tal que $\left|z-z_{0}\right|<\delta$ então a solução $\xi_{z}$ de (8.3.33) por $z$ existe em $\left[0, \tau_{n_{1}}\right]$ e satisfaz

$$
\sup _{0 \leq t \leq \tau_{n_{1}}}\left|\xi_{z}(t)-\xi_{0}(t)\right|<\eta
$$

Mas, por um lado, do Teorema do Valor Médio em conjunto com (8.3.34), (8.3.35) e (8.3.36), para $n$ suficientemente grande, temos

$$
G\left(\xi_{z_{n}}(t)\right) \leq G\left(z_{n}\right)-t c(K) m_{K} \text {, qualquer que seja } t \in\left[0, \tau_{n_{1}}\right]
$$


Por outro lado, como $\tau_{n} \in\left[0, \tau_{n_{1}}\right]$ para $n$ suficientemente grande, vemos, de (8.3.37) acima, que $G\left(\xi_{z_{n}}\left(t^{\prime}\right)\right)$ deve se anular em algum $t^{\prime} \in\left[0, \tau_{n_{1}}\right]$, e por isso, pela escolha do $\eta, \xi_{z_{n}}\left(t^{\prime}\right) \in L_{1} \cap B_{\varepsilon}$, como afirmamos. O caso para $L_{2}$ se faz analogamente.

Fixemos então, $P_{1}$ e $P_{2}$ em $H$ e $\xi_{1}$ e $\xi_{2}$ as respectivas soluções passando por estes pontos segundo a afirmação anterior. Seja $J_{0}$ o traço de uma curva em $H$ que conecta os pontos $P_{1}$ e $P_{2}$ e não intersepta as curvas $\xi_{1}$ e $\xi_{2}$ em nenhum outro ponto. Sejam também $J_{i}:=\left\{\xi_{i}(t): t \in\left[0, t_{i}\right]\right\}$ onde $t_{i}$ é o instante tal que $\xi_{i}\left(t_{i}\right) \in L_{i}, i=1,2$. Ponhamos $J^{*}:=J_{0} \cup J_{1} \cup J_{2}$.

Agora, seja

$$
\delta:=\min \left\{G(u): u \in J_{0}\right\}>0 .
$$

Como $G(u)=0$ se, e somente se, $u$ está no compacto $\overline{B_{0}}$, é possível escolher um ângulo $\alpha>0$ de maneira que o segmento de reta

$$
I_{\alpha}:=\left\{(\rho \cos \alpha, \rho \sin \alpha): l_{1}(\alpha)<\rho<l_{2}(\alpha)\right\}
$$

não intersepta o compacto $J^{*}$ e tem-se

$$
\max \left\{G(u): u \in I_{\alpha}\right\}<\delta
$$

Por esta escolha de $\alpha$, pelo decrescimento de $G$ ao longo de soluções e pela unicidade de soluções para (8.3.33), vemos que toda solução que começa em um ponto $u \in I_{\alpha}$ nunca intersepta $J$. Assim, uma tal solução ou permanece em $H$ "por toda sua vida" ou deixa o conjunto $H$ por meio de um dos dois conjuntos $L_{1} \cap B_{\varepsilon}$ ou $L_{2} \cap B_{\varepsilon}$. Afirmamos que existe um ponto $u_{0} \in I_{\alpha}$ tal que a primeira alternativa é correta.

De fato, sejam, para $i=1,2$

$$
I_{\alpha}^{i}:=\left\{u \in I_{\alpha}: \text { a solução passando por } u \text { deixa } G \text { por } L_{i} \cap B_{\varepsilon}\right\} \text {. }
$$

Repetindo (para os dois pontos da fronteira de $I_{\alpha}$ ) a mesma construção que fizemos anteriormente para garantir a existência dos pontos $P_{1}$ e $P_{2}$, conclui-se que os conjuntos $I_{\alpha}^{1}$ e $I_{\alpha}^{2}$ são não vazios. Pela continuidade com respeito as condições iniciais, não é difícil ver que $I_{\alpha}^{1}$ e $I_{\alpha}^{2}$ são ambos conjuntos abertos em $I_{\alpha}$ e, como eles possuem interseção vazia, sua reunião não pode ser todo o conjunto conexo $I_{\alpha}$, donde deve existir $u_{0} \in I_{\alpha}$ cuja solução de (8.3.33) passando por ele, enquanto existir, deve permanecer em $H$. Portanto é limitada e deve existir para todo $t \geq 0$, consequentemente, seu conjunto $\omega-$ limite é não vazio e contém apenas pontos de equilíbrio, como não existem equilíbrios de (8.3.33) em $H$, necessariamente $\omega\left(u_{0}\right) \subset S$ e é fácil comprovar que, na verdade, $\omega\left(u_{0}\right)=S$, complentando a demonstração. 
Observação 8.3.5. Observemos que a existência de uma função $G: \mathbb{R}^{2} \rightarrow \mathbb{R}$ satisfazendo as exigências da proposição acima não contradiz o Teorema 8.3.3. Com efeito, seja $u:[0, \infty) \rightarrow \mathbb{R}^{2}$ a solução obtida na conclusão da proposição anterior e escolhamos arbitrariamente $\varphi \in \omega\left(u_{0}\right)=S$. Tem-se que $L:=M^{\prime}(\varphi)=D^{2} G(\varphi)=0$ (simplesmente porque $M(u)=0$ para toda $u \in B_{0}$ e $M^{\prime}$ é contínua), $\log 0 d=\operatorname{dim} \operatorname{Ker}(L)=2$ e então, segue-se imediatamente que a hipótese $(i i)$ do Teorema 8.2.1 não se verifica para $\varphi$, pois não se pode conseguir (pelo "Brouwer Domain Invariance Theorem", veja [23]) um aberto $U \subset \mathbb{R}^{2}$ que seja homeomorfo a um conjunto $U^{\prime} \subset \overline{B_{0}}=\left\{u \in B_{\varepsilon}: M(u)=0\right\}$, de forma que $\varphi \in U^{\prime}$ (pois $U^{\prime}$ seria necessariamente um aberto de $\mathbb{R}^{2}$ e (P3) diz que não existem zeros de $M$ fora de $\overline{B_{0}}$ ), e assim nossa afirmação segue.

Contudo, deixemos aqui registrado que existe uma função $G$ satisfazendo as condições da Proposição 8.3.4, por exemplo, a função que a seguir apresentamos e que pode ser encontrada em [25] em uma versão mais geral.

Seja $G: \mathbb{R}^{2} \rightarrow \mathbb{R}$ a função definida por

$$
G(x, y):= \begin{cases}\exp \left(\frac{1}{1-|(x, y)|}\right) \sin \left(\frac{1}{|(x, y)|-1}-\gamma(x, y)\right), & |(x, y)|>1 \\ 0, & |(x, y)| \leq 1\end{cases}
$$

onde $\gamma$ é uma função suave que associa a cada $(x, y) \in \mathbb{R}^{2}$ o ângulo cuja tangente é $\frac{y}{x}$. Observemos que, ainda que esta função $\gamma$ não esteja univocamente determinada, o valor que ela informa na construção de $G$ é "irrelevante", por conta da $2 \pi$ periodicidade da função seno que aparece na formulação de $G$, e por isto $G$ resulta $C^{\infty}$.

É fácil, além disso, comprovar que a função $G$ satisfaz as hipóteses da Proposição 8.3.4, com

$$
\begin{aligned}
& R(\gamma)=1, \gamma \in \mathbb{R} \\
& l_{1}(\gamma)=1+\frac{1}{\gamma+\pi}, \gamma>0 y \\
& l_{2}(\gamma)=1+\frac{1}{\gamma}, \gamma>0 .
\end{aligned}
$$

\subsubsection{Equações de segunda ordem}

Finalizamos a parte analítico funcional deste trabalho, mostrando que é possível estender os resultados desenvolvidos na subseção anterior, relacionados a equações de primeira ordem, de forma bastante simples e utilizando as tecnicas apresentadas em [24], para certas equações de segunda ordem. 
Para isso, sejam $H$ um espaço de Hilbert com produto escalar $(\cdot, \cdot)_{H} \mathrm{e}-A: D(A) \subset$ $H \rightarrow H$ um operador setorial em $H$ (confira [14]), possuindo resolvente compacto. Sejam $H^{\alpha}$, com $\alpha \geq 0$, os espaços de potência fracionária associados a este operador, com respectivos produtos escalares $(\cdot, \cdot)_{H^{\alpha}}$, então, como sabemos, para todo $\alpha>0$, a inclusão contínua $H^{\alpha} \hookrightarrow H$ é, na verdade, compacta.

Denotemos por $V$ o espaço $H^{\frac{1}{2}}$, de maneira que $D(A)=: H^{1} \hookrightarrow V$.

Sejam agora $f: V \rightarrow H$ uma aplicação suficientemente regular, $\beta>0$ e consideremos o problema semilinear de segunda ordem

$$
\left\{\begin{array}{l}
\ddot{u}+\beta \dot{u}=A u+f(u), t>0 \\
u(0)=u_{0} \in V \\
\dot{u}(0)=u_{1} \in H
\end{array}\right.
$$

para o qual supomos a existência de uma função $G \in C^{2}(V ; \mathbb{R}) \operatorname{com} M:=G^{\prime}: V \rightarrow V^{\prime}$ satisfazendo

$$
-M(u)=A u+f(u)
$$

sempre que $u \in D(A)$.

Por outro lado, observemos que o problema (8.3.38) acima, pode escrever-se como uma equação semilinear de primeira ordem (onde supomos, sem perda de generalidade, que se tem $\beta=1$, porque o caso geral se converte neste simplesmente fazendo-se a mudança de variáveis $\left.s=\frac{t}{\eta}\right)$ :

$$
\left\{\begin{array}{c}
\dot{z}=A_{0} z+f_{0}(z) \\
z(0)=z_{0} \in Z
\end{array},\right.
$$

onde $z \in Z:=V \times H$, estando $Z$ dotado do produto escalar

$$
\left(\left(v_{1}, v_{2}\right),\left(w_{1}, w_{2}\right)\right)_{Z}:=\left(v_{1}, w_{1}\right)_{V}+\left(v_{2}, w_{2}\right)_{H}, v_{1}, w_{1} \in V, v_{2}, w_{2} \in H,
$$

$A_{0}: D\left(A_{0}\right) \subset Z \rightarrow Z$ é o operador linear com $D\left(A_{0}\right):=D(A) \times V$ e definido formalmente por

$$
A_{0}:=\left(\begin{array}{cc}
0 & I \\
A & -I
\end{array}\right)
$$

sendo $I$ a aplicação identidade de $V$ e $f_{0}: Z \rightarrow Z$ está dada por $f_{0}(z):=f_{0}(u, v):=$ $(0, f(u))$, para $z=(u, v) \in Z=V \times H$.

Sabemos que, nestas condições, o operador linear $A_{0}$ gera um $C_{0}$ semigrupo de contrações em $Z$ (confira [13]) e, então, o problema (8.3.39) tem a si associado um semigrupo, $\{S(t): t \geq 0\}$, em $Z$ como seu operador solução (porque estamos supondo que a aplicação $f: V \rightarrow H$ é suficientemente regular).

Agora, observemos que para todo $x^{*} \in H=H^{\prime} \hookrightarrow V^{\prime}$ e todo $y^{*} \in D\left(A^{*}\right)\left(A^{*}\right.$ : $D\left(A^{*}\right) \subset H \rightarrow H$ indica o operador adjunto de $\left.A\right)$ o funcional linear $z^{*}=:\left(x^{*}, y^{*}\right) \in Z^{\prime}$ 
dado por $\left\langle z^{*},(u, v)\right\rangle_{Z^{\prime}, Z}:=\left\langle x^{*}, u\right\rangle_{V^{\prime}, V}+\left(y^{*}, v\right)_{H}$, está no domínio do operador adjunto de $A_{0}$ e não é difícil ver que para todo $(u, v) \in Z=V \times H$ tem-se

$$
\left\langle A_{0}^{*} z^{*},(u, v)\right\rangle_{Z^{\prime}, Z}=\left(x^{*}, v\right)_{H}+\left(A^{*} y^{*}, u\right)_{H}-\left(y^{*}, v\right)_{H} .
$$

Nestas condições é possível provar a seguinte extensão do Teorema 8.3.3

Teorema 8.3.6. Suponhamos que as hipóteses do Teorema 8.2.1 verificam-se para toda $\varphi \in \mathcal{S}:=\{\varphi \in D(A):-A \varphi=f(\varphi)\}$, com os espaços de Hilbert $V$ e $H$ e aplicações $G$ e $M$, como estão descritas acima.

Se $u: \mathbb{R} \rightarrow V$ é solução global (em sentido estrito) para (8.3.38) com órbita $\{(u(t), \dot{u}(t)): t \in \mathbb{R}\}$ relativamente compacta em $Z=V \times H$ e $\left\|M^{\prime}(u(t)) \dot{u}(t)\right\|_{V^{\prime}} \leq$ $a\|\dot{u}(t)\|_{V^{\prime}}$ para todo real $t$ e algum a $>0$, então existem $\varphi$ e $\psi \in \mathcal{S}$ tais que

$$
\begin{gathered}
\lim _{t \rightarrow-\infty}\|u(t)-\varphi\|_{V}=0, \lim _{t \rightarrow-\infty}\|\dot{u}(t)\|_{H}=0, \\
\lim _{t \rightarrow \infty}\|u(t)-\psi\|_{V}=0 \text { e } \lim _{t \rightarrow \infty}\|\dot{u}(t)\|_{H}=0 .
\end{gathered}
$$

Demonstração: Como antes, nos limitaremos em fazer somente o caso em que $t \rightarrow-\infty$, porque o caso $t \rightarrow \infty$ pode ser feito utilizando-se argumentos semelhantes (veja [24]).

Nestas condições, definamos a função contínua $E: V \times H \rightarrow \mathbb{R}$ pondo

$$
E\left(u_{0}, u_{1}\right):=\frac{1}{2}\left\|u_{1}\right\|_{H}^{2}+G\left(u_{0}\right) .
$$

Queremos provar que $E$ é uma função de Lyapunov para o semigrupo $\{S(t): t \geq 0\}$ associado ao problema (8.3.39).

Para isso, seja $\left(u_{0}, u_{1}\right) \in D\left(A_{0}\right)$ um dado inicial qualquer, então o caminho $[0, \infty) \ni$ $t \mapsto S(t)\left(u_{0}, u_{1}\right) \in V \times H$ define uma solução estrita de (8.3.39), portanto é da forma $S(t)\left(u_{0}, u_{1}\right)=(w(t), \dot{w}(t)) t \geq 0$, onde $w:[0, \infty) \rightarrow V$ é um caminho (possuindo derivada primeira contínua tomando valores em $V$ e derivada segunda contínua quando vista assumindo valores em $H$ ), de modo que podemos calcular a derivada de $E$ ao longo da solução $(w, \dot{w})$ para obtermos, para todo $t>0$, por meio da regra da cadeia, que

$$
\begin{gathered}
\frac{d}{d t} E(w, \dot{w})(t)=(\ddot{w}(t), \dot{w}(t))_{H}+\langle M(w(t)), \dot{w}(t)\rangle_{V^{\prime}, V} \\
=(\ddot{w}(t)+M(w(t)), \dot{w}(t))_{H}=-\|\dot{w}(t)\|_{H}^{2} .
\end{gathered}
$$

Por outro lado, seja $\left(u_{0}, w_{0}\right) \in V \times H$ um dado arbitrário e consideremos o caminho $[0, \infty) \ni t \rightarrow S(t)\left(u_{0}, w_{0}\right)=:\left(u_{0}(t), w_{0}(t)\right) \in V \times H$, que é a solução por ele, onde, evidentemente, $u_{0}(t):=P_{V} S(t)\left(u_{0}, w_{0}\right)$ e $w_{0}(t):=P_{H} S(t)\left(u_{0}, w_{0}\right)$ (sendo $P_{V}: V \times H \rightarrow$ $V$ e $P_{H}: V \times H \rightarrow H$, as projeções correspondentes). 
Sejam agora, $\left(\left(u_{n}, w_{n}\right)\right)_{n \in \mathbb{N}}$ uma sequência em $D\left(A_{0}\right)$ com

$$
\lim _{n \rightarrow \infty}\left\|u_{n}-u_{0}\right\|_{V}=0, \lim _{n \rightarrow \infty}\left\|w_{n}-w_{0}\right\|_{H}=0
$$

e

$$
[0, \infty) \ni t \rightarrow S(t)\left(u_{n}, w_{n}\right)=:\left(u_{n}(t), w_{n}(t)\right) \in V \times H
$$

as soluções correspondentes (com as mesmas considerações de antes sobre as projeções). Sejam também, para $n=0,1,2, \cdots$ e $t \geq 0, E_{n}(t):=E\left(u_{n}(t), w_{n}(t)\right)$. Da continuidade do semigrupo segue-se que $\left\|w_{n}(t)-w_{0}(t)\right\|_{H} \rightarrow 0$, quando $n \rightarrow \infty$, uniformemente para $t$ em compactos de $[0, \infty)$. O que, usando também a continuidade da função $G$, nos mostra que

$$
E_{n}(t) \underset{n \rightarrow \infty}{\rightarrow} E_{0}(t) \text { e } \frac{d}{d t} E_{n}(t)=-\left\|w_{n}(t)\right\|_{H}^{2} \underset{n \rightarrow \infty}{\rightarrow}-\left\|w_{0}(t)\right\|_{H}^{2}
$$

uniformemente para $t$ em compactos de $[0, \infty)$, portanto a função real $E_{0}$ é continuamente diferenciável com

$$
\frac{d}{d t} E_{0}(t)=-\left\|w_{0}(t)\right\|_{H}^{2} \leq 0, \text { qualquer que seja } t>0 .
$$

Donde $E$ é não crescente ao longo de todas as soluções do semigrupo.

A igualdade (8.3.41) acima também nos diz que, se para certos $\left(u_{0}, w_{0}\right) \in V \times H$ e $t_{0} \geq 0$ temos $E\left(S(t)\left(u_{0}, w_{0}\right)\right)=E\left(u_{0}, w_{0}\right)$ para todo $t \geq t_{0}$ então

$$
S(t)\left(u_{0}, w_{0}\right)=\left(u_{0}(t), 0\right) \text { para todo } t \geq 0 .
$$

Mostremos que esta última igualdade implica que $u_{0}:[0, \infty) \rightarrow V$ deve ser uma solução de equilíbrio para a equação

$$
\dot{u}=A u+f(u),
$$

e portanto $(-\infty, 0) \ni t \mapsto\left(u_{0}(t), 0\right) \in V \times H$ será um equilíbrio de (8.3.39).

Para concluirmos esta tarefa, usaremos a caracterização para soluções fracas ("mildes" em Inglês) de problemas semilineares abstratos, que se encontra em [26].

Em conformidade com dita referência, para todo funcional $z^{*} \in D\left(A_{0}^{*}\right)$ e todo $t>0$ temos

$$
\frac{d}{d t}\left\langle z^{*},\left(u_{0}(t), 0\right)\right\rangle_{Z^{\prime}, Z}=\left\langle A_{0}^{*} z^{*},\left(u_{0}(t), 0\right)\right\rangle_{Z^{\prime}, Z}+\left\langle z^{*}, f_{0}\left(u_{0}(t), 0\right)\right\rangle_{Z^{\prime}, Z},
$$

onde a equação é entendida em sentido estrito.

Em particular, dado um funcional $y^{*} \in D\left(A^{*}\right)$, temos que para todo $t>0$ (com $y^{*}$ visto como funcional contínuo em $Z$ conforme explicamos anteriormente)

$$
\frac{d}{d t}\left\langle\left(0, y^{*}\right),\left(u_{0}(t), 0\right)\right\rangle_{Z^{\prime}, Z}=\left\langle A_{0}^{*}\left(0, y^{*}\right),\left(u_{0}(t), 0\right)\right\rangle_{Z^{\prime}, Z}+\left\langle\left(0, y^{*}\right), f_{0}\left(u_{0}(t), 0\right)\right\rangle_{Z^{\prime}, Z} .
$$


Logo, por um lado, levando em conta (8.3.40), para todo $t>0$ temos

$$
0=\left(A^{*} y^{*}, u_{0}(t)\right)_{H}+\left(y^{*}, f\left(u_{0}(t)\right)\right)_{H} .
$$

Por outro, se $x^{*} \in H=H^{\prime} \hookrightarrow V^{\prime}$ então, para todo $t>0$

$$
\frac{d}{d t}\left\langle\left(x^{*}, 0\right),\left(u_{0}(t), 0\right)\right\rangle_{Z^{\prime}, Z}=\left\langle A_{0}^{*}\left(x^{*}, 0\right),\left(u_{0}(t), 0\right)\right\rangle_{Z^{\prime}, Z}+\left\langle\left(x^{*}, 0\right),\left(0, f\left(u_{0}(t)\right)\right)\right\rangle_{Z^{\prime}, Z},
$$

então, também por (8.3.40),

$$
\frac{d}{d t}\left(x^{*}, u_{0}(t)\right)=\left(x^{*}, 0\right)_{H}+\left(A^{*} 0, u_{0}(t)\right)_{H}-(0,0)_{H}+\left(x^{*}, 0\right)_{H}+\left(0, f\left(u_{0}(t)\right)\right)_{H}=0 .
$$

Reunindo, (8.3.42) e (8.3.43), conclui-se que para todo $x^{*} \in D\left(A^{*}\right)$ e todo $t>0$ tem-se

$$
\frac{d}{d t}\left(x^{*}, u_{0}(t)\right)=\left(A^{*} x^{*}, u_{0}(t)\right)_{H}+\left(x^{*}, f\left(u_{0}(t)\right)\right)_{H}=0,
$$

mostrando que $u_{0}:[0, \infty) \rightarrow V$ é solução fraca para $\dot{u}=A u+f(u)$.

Mas, como $\frac{d}{d t}\left(x^{*}, u_{0}(t)\right)=0$ para todo $t>0$ e todo $x^{*} \in D\left(A^{*}\right)$, resulta que para cada $x^{*} \in D\left(A^{*}\right)$ a função real $[0, \infty) \ni t \longmapsto\left(x^{*}, u_{0}(t)\right) \in \mathbb{R}$ deve ser constante. Como o conjunto $D\left(A^{*}\right)$ é denso em $H^{\prime}$ (confira [22]) conclui-se que o caminho $u_{0}:[0, \infty) \rightarrow V$ deve ser constante, $\log 0 u_{0}(t)=\varphi$ para todo $t \geq 0$, onde $\varphi \in V$ satisfaz $-A \varphi=f(\varphi)$, como afirmamos.

Agora, evidentemente, o par $(\varphi, 0)$ é um equilíbrio para (8.3.39), donde $E: V \times H \rightarrow$ $\mathbb{R}$ é, de fato, uma função de Lyapunov para o semigrupo $\{S(t): t \geq 0\}$.

Por outro lado, a hipótese de compacidade da solução $u: \mathbb{R} \rightarrow V$ nos dá que o conjunto $\alpha$-limte da solução $u$,

$$
\begin{gathered}
\alpha(u):=\left\{(z, w) \in V \times H: \text { existe } t_{n} \rightarrow-\infty\right. \text { com } \\
\left.\lim _{n \rightarrow \infty}\left\|u\left(t_{n}\right)-z\right\|_{V}=0 \text { e } \lim _{n \rightarrow \infty}\left\|\dot{u}\left(t_{n}\right)-w\right\|_{H}=0\right\},
\end{gathered}
$$

é não vazio, compacto, invariante, atrai a solução $(u, \dot{u})$ em $V \times H$ quando $t \rightarrow-\infty$ e, sendo o semigrupo $S(\cdot)$ um semigrupo gradiente, está contido no conjunto

$$
\mathcal{E}^{*}:=\left\{\left(z^{*}, w^{*}\right) \in V \times H: S(t)\left(z^{*}, w^{*}\right)=\left(z^{*}, w^{*}\right) \text { para todo } t \geq 0\right\} .
$$

Sabemos que as soluções de equilíbrio do problema (8.3.39) são soluções estritas do mesmo, então, necessariamente, para todo $\left(z^{*}, w^{*}\right) \in \mathcal{E}^{*}$ tem-se $w^{*}=0$ e $M\left(z^{*}\right)=0$, ou seja, $\mathcal{E}^{*} \subset \mathcal{S} \times\{0\}$ (portanto, tem-se de fato a igualdade $\mathcal{E}^{*}=\mathcal{S} \times\{0\}$, uma vez que a inclusão que falta, $\mathcal{E}^{*} \supset \mathcal{S} \times\{0\}$, é sempre verdadeira), em particular, $\lim _{t \rightarrow-\infty}\|\dot{u}(t)\|_{H}=0$. 
Provemos agora que existe um único $\varphi \in \mathcal{S}$ tal que $\alpha(u)=\{(\varphi, 0)\}$, o que completará a demonstração.

Com efeito, seja $\varepsilon$ um número real positivo arbitrário e definamos, para $t \leq 0$

$$
E_{\varepsilon}(t):=E(u(t), \dot{u}(t))+\varepsilon(M(u(t)), \dot{u}(t))_{V^{\prime}} .
$$

Derivando vem (antes, observemos que nos é permitido aplicar a regra da cadeia usual, para calcular a derivada da composição $(-\infty, 0) \ni t \mapsto M(u(t)) \in V^{\prime}$ no cálculo que segue, porque estamos supondo que $u: \mathbb{R} \rightarrow V$ é solução estrita para (8.3.38), ou seja, a aplicação $z: \mathbb{R} \rightarrow V \times H$ dada por $z(t):=(u(t), \dot{u}(t)), t \leq 0$, é continuamente diferenciável tomando valores em $V \times H$ e verifica a equação (8.3.39) em sentido clássico),

$$
\begin{gathered}
\dot{E}_{\varepsilon}(t):=-\|\dot{u}(t)\|_{H}^{2}+\varepsilon\left[\left(M^{\prime}(u(t)) \dot{u}(t), \dot{u}(t)\right)_{V^{\prime}}+(M(u(t)), \ddot{u}(t))_{V^{\prime}}\right] \\
=-\|\dot{u}(t)\|_{H}^{2}+\varepsilon\left[\left(M^{\prime}(u(t)) \dot{u}(t), \dot{u}(t)\right)_{V^{\prime}}+(M(u(t)),-M(u(t))-\dot{u}(t))_{V^{\prime}}\right] \\
=-\|\dot{u}(t)\|_{H}^{2}+\varepsilon\left[\left(M^{\prime}(u(t)) \dot{u}(t), \dot{u}(t)\right)_{V^{\prime}}-\|M(u(t))\|_{V^{\prime}}^{2}-(M(u(t)), \dot{u}(t))_{V^{\prime}}\right] \\
\leq-\|\dot{u}(t)\|_{H}^{2}+ \\
\varepsilon\left[\left\|M^{\prime}(u(t)) \dot{u}(t)\right\|_{V^{\prime}}\|\dot{u}(t)\|_{V^{\prime}}-\|M(u(t))\|_{V^{\prime}}^{2}+\|M(u(t))\|_{V^{\prime}}\|\dot{u}(t)\|_{V^{\prime}}\right] .
\end{gathered}
$$

Da hipótese $\left\|M^{\prime}(u(t)) \dot{u}(t)\right\|_{V^{\prime}} \leq a\|\dot{u}(t)\|_{V^{\prime}}$ para todo real $t$ e algum $a>0$, usando as inclusões contínuas $V \hookrightarrow H \hookrightarrow V^{\prime}$ e a continuidade de $M^{\prime}$ segue-se a existência de uma constante $C>0$ tal que (usando também a desigualdade de Young) (8.3.44) resulta menor do que ou igual a

$$
\begin{gathered}
-\|\dot{u}(t)\|_{H}^{2}+\varepsilon\left[C\|\dot{u}(t)\|_{H}^{2}-\|M(u(t))\|_{V^{\prime}}^{2}+\frac{1}{2}\|M(u(t))\|_{V^{\prime}}^{2}+\frac{C}{2}\|\dot{u}(t)\|_{H}^{2}\right] \\
=-\|\dot{u}(t)\|_{H}^{2}+\varepsilon\left[\left(C+\frac{C}{2}\right)\|\dot{u}(t)\|_{H}^{2}-\frac{1}{2}\|M(u(t))\|_{V^{\prime}}^{2}\right] \\
=\left[\varepsilon\left(C+\frac{C}{2}\right)-1\right]\|\dot{u}(t)\|_{H}^{2}-\frac{\varepsilon}{2}\|M(u(t))\|_{V^{\prime}}^{2} .
\end{gathered}
$$

Donde conclui-se que

$$
\dot{E}_{\varepsilon}(t) \leq\left[\varepsilon\left(C+\frac{C}{2}\right)-1\right]\|\dot{u}(t)\|_{H}^{2}-\frac{\varepsilon}{2}\|M(u(t))\|_{V^{\prime}}^{2},
$$

mostrando que se $\varepsilon>0$ é suficientemente pequeno a função $E_{\varepsilon}:(-\infty, 0] \rightarrow \mathbb{R}$ é não crescente (observemos também, que $\varepsilon>0$ depende de $u$ para esta conclusão) e como é também limitada (isto segue-se da hipótese de pré compacidade da órbita de $u$ ) existe o limite $\lim _{t \rightarrow-\infty} E_{\varepsilon}(t)$. 
Fixemos pois um tal $\varepsilon>0$.

Tomando $(\varphi, 0) \in \alpha(u)$, existe uma sequência $\left(t_{n}\right)_{n \in \mathbb{N}} \operatorname{com} t_{n} \rightarrow-\infty$ tal que

$$
\lim _{n \rightarrow \infty}\left\|u\left(t_{n}\right)-\varphi\right\|_{V}=0
$$

donde

$$
\lim _{n \rightarrow \infty} E_{\varepsilon}\left(t_{n}\right)=\lim _{n \rightarrow \infty}\left[\frac{1}{2}\left\|\dot{u}\left(t_{n}\right)\right\|_{H}^{2}+G\left(u\left(t_{n}\right)\right)+\varepsilon\left(M\left(u\left(t_{n}\right)\right), \dot{u}\left(t_{n}\right)\right)_{V^{\prime}}\right]=G(\varphi),
$$

e assim, $\lim _{t \rightarrow-\infty} E_{\varepsilon}(t)=G(\varphi)$ com $E_{\varepsilon}(t) \leq G(\varphi)$ para todo $t \leq 0$.

Por outro lado, de (8.3.45), para alguma constante positiva $C_{1}$, vem

$$
\dot{E}_{\varepsilon}(t) \leq-\frac{1}{C_{1}}\|\dot{u}(t)\|_{H}^{2}
$$

o que integrando de $-\infty$ até $t \in(-\infty, 0)$ nos dá

$$
\int_{-\infty}^{t}\|\dot{u}(s)\|_{H}^{2} d s \leq C_{1}\left[G(\varphi)-E_{\varepsilon}(t)\right]
$$

Agora, para cada $j \in \mathbb{N}$ escolhamos, como na prova do Teorema 8.3.3, um natural $n_{j}$ de maneira que $n_{1}<n_{2}<\cdots$ com

$$
\left\|u\left(t_{n_{j}}\right)-\varphi\right\|_{V}<\frac{1}{j} \mathrm{e}\left[G(\varphi)-E_{\varepsilon}(t)\right]^{\frac{1}{2}}<\frac{1}{j} .
$$

Analogamente, sejam $\sigma>0$ associado ao equilíbrio $\varphi$ satisfazendo a conclusão do Teorema 8.2.1, $j_{0} \in \mathbb{N}$ tal que $\frac{1}{j_{0}}<\sigma$ e definamos para $j \geq j_{0}$

$$
\bar{t}_{j}:=\inf \left\{\tau<t_{n_{j}}:\|u(t)-\varphi\|_{V}<\sigma \text { para todo } t \in\left[\tau, t_{n_{j}}\right]\right\} .
$$

Então, para cada $j \geq j_{0}, t \in\left[\bar{t}_{j}, t_{n_{j}}\right]$ e usando a Desigualdade de Lojasiewicz-Simon escrevamos

$$
\begin{aligned}
0 \geq E_{\varepsilon}(t) & -G(\varphi)=\frac{1}{2}\|\dot{u}(t)\|_{H}^{2}+\varepsilon(M(u(t)), \dot{u}(t))_{V^{\prime}}+[G(u(t))-G(\varphi)] \\
& \geq \frac{1}{2}\|\dot{u}(t)\|_{H}^{2}+\varepsilon(M(u(t)), \dot{u}(t))_{V^{\prime}}-c\|M(u(t))\|_{V^{\prime}}^{2}
\end{aligned}
$$

pela desigualdade de Young, para todo $\delta>0$ temos

$$
\left|(M(u(t)), \dot{u}(t))_{V^{\prime}}\right| \leq\|M(u(t))\|_{V^{\prime}}\|\dot{u}(t)\|_{V^{\prime}} \leq \frac{1}{2 \delta}\|M(u(t))\|_{V^{\prime}}^{2}+\frac{\delta}{2}\|\dot{u}(t)\|_{V^{\prime}}^{2},
$$

o que aplicado a (8.3.47) nos dá

$$
E_{\varepsilon}(t)-G(\varphi) \geq \frac{1}{2}\|\dot{u}(t)\|_{H}^{2}-c\|M(u(t))\|_{V^{\prime}}^{2}-\frac{\varepsilon}{2 \delta}\|M(u(t))\|_{V^{\prime}}^{2}-\frac{\varepsilon \delta}{2}\|\dot{u}(t)\|_{V^{\prime}}^{2},
$$


escolhendo então $\delta>0$ suficientemente grande, encontramos uma constante positiva $C_{1}>0$ tal que

$$
E_{\varepsilon}(t)-G(\varphi) \geq-C_{1}\left[\|\dot{u}(t)\|_{H}^{2}+\|M(u(t))\|_{V^{\prime}}^{2}\right] \text {, para todo } t \in\left[\bar{t}_{j}, t_{n_{j}}\right]
$$

Obserevemos agora que, por (8.3.45), para uma certa constante $C_{2}>0$ e qualquer que seja $t<0$ temos

$$
\frac{d}{d t}\left[E_{\varepsilon}(t)-G(\varphi)\right] \leq-C_{2}\left[\|\dot{u}(t)\|_{H}^{2}+\|M(u(t))\|_{V^{\prime}}^{2}\right]
$$

o que em conjunto com (8.3.48) acima nos mostra que se $t \in\left[\bar{t}_{j}, t_{n_{j}}\right]$ então

$$
\frac{d}{d t}\left[E_{\varepsilon}(t)-G(\varphi)\right] \leq \gamma\left[E_{\varepsilon}(t)-G(\varphi)\right]
$$

onde $\gamma:=\frac{C_{2}}{C_{1}}$

Aplicando-se pois o mesmo procedimento que o realizado na prova do Teorema 8.3.3, para todo $t \in\left[\bar{t}_{j}, t_{n_{j}}\right]$, obtém-se que

$$
\left[G(\varphi)-E_{\varepsilon}(t)\right] \leq\left[G(\varphi)-E_{\varepsilon}\left(t_{n_{j}}\right)\right] e^{\gamma\left(t-t_{n_{j}}\right)},
$$

o que agregado a (8.3.46) nos diz que se $t \in\left[\bar{t}_{j}, t_{n_{j}}\right]$, então

$$
\int_{-\infty}^{t}\|\dot{u}(s)\|_{H}^{2} d s \leq C_{1}\left[G(\varphi)-E_{\varepsilon}\left(t_{n_{j}}\right)\right] e^{\gamma\left(t-t_{n_{j}}\right)} .
$$

Desta forma, estamos novamente em condições de aplicar o Lema 8.3.1 e concluir a demonstração deste teorema com os mesmos argumentos usados para terminar a prova do Teorema 8.3.3, completando a demonstração. 



\section{Capítulo}

\section{Conclusões e problemas em aberto}

Neste trabalho, em um primeiro momento, estabelecemos a equivalência entre as duas noções de semigrupos estudadas, ou seja, a equivalência entre os conceitos de semigrupos gradiente e de tipo gradiente. Uma vez provada tal equivalência, segundo o Teorema 3.2.4, que foi apresentado pela primeira vez em [8], conclui-se a estabilidade dos semigrupos gradientes por perturbação. Este foi nosso objetivo principal, no contexto dos sistemas dinâmicos autônomos, e teve como resultado o trabalho apresentado em [1].

Outro resultado importante que estabelecemos se refere à estabilidade, por perturbação, da função de Lyapunov que construímos para os semigrupos de tipo gradiente. O que também é um resultado novo e compõe parte do conteúdo do trabalho [2].

Um fato importante que desenvolvemos nos trabalhos mencionados acima, e apresentados em detalhes neste texto, é o uso da teoria de Morse na solução dos dois problemas principais que aqui analizamos. Unir a teoria de Morse à teoria dos semigrupos de tipo gradiente, o que se concretizou, basicamente, no Lema 4.3.2, foi o fator decisivo que permitiu estabelecer o resultado de equivalência contido no Teorema 4.4.1, por meio do Teorema 4.3.3 junto com a Proposição 4.2.2.

Também introduzimos um novo conceito dinâmico para ajudar na descrição dos atratores globais, o conceito de níveis de energia (confira a Definição 6.1.1), que apresenta uma maneira de organizar os pontos de equilíbrio (ou mais geralmente, conjuntos invariantes isolados), de um sistema autônomo, que possuem propriedades dinâmicas semelhantes, reunindo aqueles pontos que, por assim dizer, produzem o mesmo efeito sobre as soluções globais que se deslocam em seus arredores. Além disso, fornece- 
mos condições suficientes para a estabilidade destes objetos (veja os Teoremas 6.2.2 e 6.2.3). Cabe observar que, embora não tenhamos apresentamos aqui, é possível entender a utilidade prática dos níveis de energia, quando se estuda, por exemplo, a dinâmica do problema clássico do atrator de Chafee-Infante (confira [9]).

Uma característica importante que possuem os níveis de energia é que introduzem uma ordem total no conjunto dos pontos de equilíbrio, ou, em outras palavras, fornecem condições dinâmicas capazes de relacionar, por meio do "fluxo", todos os pontos de equilíbrio de um sistema de tipo gradiente, conforme nos mostrou o Lema 6.1.2.

Como uma segunda abordagem sobre a existência de funções de Lyapunov, fomos um pouco mais adiante e nos perguntamos também sobre uma tal possibilidade no caso não autônomo e também, neste contexto, foi possível concluir que os processos de evolução de tipo gradiente (confira [8]) possuem uma função de Lyapunov construída com base em sua dinâmica (veja o Teorema 7.4.7) sendo, como o que ocorreu no caso autônomo, a descrição precisa dos conjuntos de Morse, que ainda não haviam sido definidos, até o momento, no caso dos processos de evolução, o fator chave para sua construção.

Observemos que, no marco não autônomo, impusemos algumas condições sobre o comportamento dos pares atrator-repulsor (por exemplo no Lema fundamental 7.3.3, para a continuidade da função de Lyapunov destes pares, e no Teorema 7.3.9, para a construção da decomposição de Morse para um processo de tipo gradiente, cujos conjuntos de Morse são determinados, efetivamente, pelas famílias invariantes isoladas que dão ao referido processo a estrutura de "tipo gradiente"). Levando em conta este fato, tivemos o cuidado de estudar separadamente alguns casos onde conclusões melhores fizeram-se possíveis (como na Proposição 7.4.4 e no Teorema 7.4.6) e também fornecer exemplos onde as restrições impostas possam ser encontradas satisfeitas, concretamente, provamos que as perturbações não autônomas pequenas de um semigrupo gradiente satisfazem as exigências que impusemos nos resultados abstratos.

Ainda neste caso de perturbações não autônomas pequenas de um semigrupo gradiente, obtivemos, baixo condições bastante razoáveis, a convergência das funções de Lyapunov dos pares atrator-repulsor dos processos perturbados, para a função de Lyapunov do semigrupo limite, estabelecendo assim a versão não autônoma da Proposição 5.2 .1 .

Estas conclusões no marco não autônomo, deram origem ao trabalho [3], já submetido para possível publicação.

Finalmente, como aplicação de alguns dos resultados abstratos que desenvolvemos, escrevemos o Capítulo 8, onde, além de estender alguns dos resultados de [24], mais precisamente, o caso onde se considera $t$ tendendo a menos infinito (não estudado no 
Teorema 1.1 de [24]), obtendo assim o Teorema 8.3.3, provamos também o Lema 8.1.6 e, com sua ajuda, o Teorema 8.1.7. Temas que compuzeram o conteúdo do trabalho [4], igualmente submetido para possível publicação.

Devemos destacar que todos os conceitos estudados neste trabalho, a pesar de seu caráter abstrato, têm sua aplicabilidade no tratamento das EDP's e das EDO's, o que constituiu o fator principal na motivação do desenvolvimento deste projeto de pesquisa a um nível abstrato. Em outras palavras, a aplicabilidade dos resultados obtidos aqui abre-se ao vasto campo de fenômenos modelados por equações diferenciais. Motivando a elaboração desta nossa Tese Doutoral.

Como questões que ainda não obtivemos respostas, estão as extensões de alguns resultados estudados, no caso autônomo, permitindo que a família de conjuntos invariantes isolados de um semigrupo de tipo gradiente (considerando-se agora a Definição 8.1.1) seja infinita. Neste caso, seria interessante abordar o problema de existência de algum tipo de "decomposição de Morse" que tenha como conjuntos de Morse tais conjuntos invarinates e também a existência de uma "função de Lyapunov" para semigrupos com esta característica. Observemos que estas extensões não têm porque serem triviais, pois em muitos argumentos por contradição que aqui desenvolvemos, a hipótese de finitude da família de conjuntos invariantes foi fundamental para se obter o absurdo (confira, apenas por citar um exemplo, a demonstração do Lema 4.3.2).

Já no caso não autônomo, uma questão de interesse, seria poder provar uma recíproca para o Teorema 7.4.7, ou seja, supondo a existência de uma função de Lyapunov para um processo, no sentido da Definição 7.4.1, provar que tal processo é de tipo gradiente no sentido da Definição 7.2.4.

Estas perguntas, certamente, abrem caminho para a elaboração de trabalhos futuros onde também teremos como intenção abordar a análise no caso de equações estocásticas, o que acreditamos dará lugar a outra interessante linha de pesquisa. 

[1] E.R. Aragão-Costa, T. Caraballo, A.N. Carvalho and J.A. Langa, Stability of gradient semigroups under perturbation, Nonlinearity 24 (2011), 2099-2117.

[2] E.R. Aragão-Costa, T. Caraballo, A.N. Carvalho and J.A. Langa, Continuity of Lyapunov functions and of energy level for a generalized gradient system, Topological Methods in Nonlinear Analysis 39 (2012), 57-82.

[3] E.R. Aragão-Costa, T. Caraballo, A.N. Carvalho and J.A. Langa, Non-Autonomous Morse Decomposition and Lyapunov functions for Gradient-like Process, Transactions of the American Mathematical Society (Aceito para publicação).

[4] E.R. Aragão-Costa, A.N. Carvalho, P. Marín-Rubio and Gabriela Planas, Gradientlike Nonlinear Semigroups with Infinitely many Equilibria and Applications to Cascade Systems, preprint.

[5] Arrieta, J.M., Carvalho, A.N. and Lozada-Cruz, G. Dynamics in dumbbell domains II. The Limiting Problem, Journal of Differential Equations, 247 (1) 174-202 (2009).

[6] A. V. Babin and M. I. Vishik, Attractors in Evolutionary Equations Studies in Mathematics and its Applications 25, North-Holland Publishing Co., Amsterdam, (1992).

[7] T. Caraballo, A. N. Carvalho, J. A. Langa and L. F. Rivero, A gradient-like nonautonomous evolution processes, International Journal of Bifurcation and Chaos, 20 (9) 2751-2760 (2010).

[8] A. N. Carvalho and J. A. Langa, An extension of the concept of gradient semigroups which is stable under perturbation, J. Differential Equations 246 (2009), 2646-2668. 
[9] N. Chafee and E. F. Infante. A bifurcation problem for a nonlinear partial differential equation of parabolic type. Applicable Anal., 4:17-37, 1974/75.

[10] A. N. Carvalho, J. A. Langa, J. C. Robinson, On the continuity of pullback attractors for evolution processes, Nonlinear Analysis 71 (2009), 1812-1824.

[11] Carvalho, A.N., Langa, J.A., Robinson, J.C., Súarez A., Characterization of nonautonomous attractors of aperturbed infinite-dimensional gradient system. J. Differential Equations, 236 (2007) 570-603.

[12] C. Conley, Isolated invariant sets and the Morse index. CBMS Regional Conference Series in Mathematics, 38. American Mathematical Society, Providence, R.I. (1978).

[13] J. K. Hale Asymptotic Behavior of Dissipative Systems, Mathematical Surveys and Monographs Number 25 (American Mathematical Society, Providence, RI) (1988).

[14] D. Henry, Geometric Theory of Semilinear Parabolic Equations, Lecture Notes in Mathematics 840, Springer-Verlag, Berlin, (1981).

[15] M. Hurley, Chain recurrence, semiflows and gradients, J. Dyn. Diff. Equations 7 (1995), 437-456.

[16] O. A. Ladyzhenskaya, Attractors for Semigroups and Evolution Equations, Cambridge University Press, Cambridge (1991).

[17] D.E. Norton, The fundamental theorem of dynamical systems, Comment.Math.,Univ.Carolinae36(3)(1995),585-597.

[18] M. Patrao, Morse decomposition of semiflows on topological spaces, J. Dyn. Diff. Equations 19 (1) (2007), 181-198.

[19] M. Patrao and Luiz A.B. San Martin, Semiflows on topological spaces: chain transitivity and semigroups, J. Dyn. Diff. Equations 19 (1) (2007), 155-180.

[20] K. P. Rybakowski, The homotopy index and partial differential equations, Universitext, Springer-Verlag (1987).

[21] R. Temam, Infinite-Dimensional Dynamical Systems in Mechanics and Physics, Springer-Verlag, Berlin (1988; second edition 1996).

[22] Pazy A. Semigroups of linear operators and applications to PDEs (Springer, 1992)

[23] Dugundji, James; Topology, Allyn and Bacon, Boston, 1965. 
[24] A. Haraux and M. A. Jendoubi, On the convergence of global and bounded solutions of some evolution equations, J. Evol. Equ. 7 (2007), 449-470.

[25] P. Polácik and F. Simondon. Nonconvergent bounded solutions of semilinear heat equations on arbitrary domains. J. Differential Equations 186 (2002), 586-610.

[26] J. M. Ball, Strongly continuous semigroups, weak solutions and the variation of constants formula, proc. Amer. Math. Soc. 63 (1977) 370-373 